\title{
Rapid Determination of High-resolution Protein Structures by Solution and Solid-state NMR Spectroscopy
}

\author{
Dissertation \\ zur Erlangung des Doktorgrades \\ der Mathematisch-Naturwissenschaftlichen Fakultäten \\ der Georg-August-Universität zu Göttingen
}

\author{
vorgelegt von \\ Jegannath Korukottu \\ aus Madurai, India
}

Göttingen 2008 
D7

Referent: Prof. Dr. Annette Zippelius

Korreferent: Prof. Dr. Christian Griesinger

Tag der mündlichen Prüfung: 


\section{Summary}

NMR spectroscopy provides high-resolution structural information of biomolecules in near-physiological conditions. Structural studies of proteins and nucleic acids are critical for understanding biological processes at the molecular level. Although significant improvements were achieved in NMR spectroscopy in the last 20 years, the increase in genome sequencing data has created a need for rapid and efficient methods of NMR-based structure determination. NMR data acquisition can be accelerated significantly, when sensitive spectrometers are combined with new methods for sampling chemical shifts in multidimensional NMR experiments. Therefore, data analysis and in particular the requirement to assign side chain chemical shifts to specific atoms is the major bottleneck of rapid NMR-based structure determination. In chapter 2, a method termed FastNMR (FAst STructure determination by $\mathbf{N M R}$ ), is described in detail, which enables automatic, highresolution NMR structure determination of domain-sized proteins starting from unassigned NMR data. Using FastNMR the de novo structure of the 65-residue cone snail neurotoxin conkunitzin-S2 was determined automatically.

Large classes of proteins, such as membrane proteins and insoluble aggregates of peptides and more complex systems, cannot be investigated with the above method, because the proteins cannot be made soluble for liquid-state NMR. Therefore, there is a considerable interest in the development of methods for protein structure determination that do not have these limitations. In chapter 3 and 4 of this thesis, it is demonstrated that, combining the knowledge obtained in solution-state NMR, a rapid determination of high-resolution protein structure of globular proteins, such as, potassium channel blocker, Kaliotoxin existing in free form and also in complex with KcsA-Kv1.3, from solid-state NMR data could be obtained. Also in chapter 4, an improved model of KTX-KcsA-Kv1.3 complex is proposed based on functional and solid-state NMR data.

Finally, chapter 5 sheds light on understanding the mechanism of alignment of proteins and efforts in improving the accuracy of prediction of charge-induced molecular alignment from the protein's known 3D structure, by employing more atomistically detailed electrostatic models. Preliminary results suggest that the accuracy in predicting RDCs and magnitude of alignment using detailed electrostatics might improve in comparison with the simplified model implemented in PALES. 


\section{Zusammenfassung}

NMR-Spektroskopie ermöglicht die Bestimmung hoch-aufgelöster Strukturen von Biomolekülen unter nahezu physiologischen Bedingungen. In den letzten 20 Jahren wurden erhebliche Fortschritte in der NMR-Spektroskopie erzielt, durch die Vielzahl sequenzierter Genome werden jedoch Hochdurchsatzverfahren zur Bestimmung der Tertiärstruktur von Proteinen immer wichtiger. Die Datenaufnahme kann erheblich beschleunigt werden, wenn moderne NMR-Spektrometer mit Methoden kombiniert werden, welche effizient chemische Verschiebungen in mehrdimensionalen NMRExperimenten messen. Daher sind die Datenanalyse und insbesondere die Notwendigkeit, chemische Verschiebungen sequenzspezifisch den Atomen der Seitenketten zuzuordnen, die Haupthindernisse für eine schnelle NMR-basierte Proteinstrukturbestimmung. In Kapitel 2 wird die Methode FastNMR (FAst STructure determination by $\mathbf{N M R}$ ) beschrieben, welche ausgehend von nicht zugeordneten NMR-Daten die automatische Bestimmung hoch-aufgelöster Strukturen von Proteinen - die aus einer Domäne bestehen - ermöglicht. Mittels FastNMR wurde automatisch die de novo Struktur des aus 65 Aminosäuren bestehenden, aus Kegelschnecken stammenden Neurotoxins Conkunitzin-S2 bestimmt.

Eine große Zahl von Proteinen, wie z.B. Membranproteine oder unlösliche Aggregate von Peptiden und komplexeren Systemen, läßt sich allerdings nicht mit den zuvor beschrieben Methoden untersuchen, da diese Proteine nicht in Lösung gebracht werden können, um mit Hilfe von Lösungs-NMR untersucht zu werden. Daher besteht ein großes Interesse an der Entwicklung von Methoden zur ProteinstrukturAufklärung, die nicht auf Lösungs-NMR beschränkt sind. In Kapitel 3 und 4 dieser Arbeit wird gezeigt, dass mit Hilfe der in Lösung-NMR gewonnen Erkenntnisse eine schnelle Aufklärung von globulären Proteinen, wie z.B. dem Kaliumkanal-Blocker Kalitoxin - in der freien Form als auch in der im Komplex mit KcsA-Kv1.3 gebunden Form - durch Festkörper-NMR möglich ist. Ebenfalls in Kapitel 4 wird ein verfeinertes Model des KTX-KcsA-Kv1.3 Komplexes vorgestellt auf der Grundlage von biochemischen Daten und Festkörper-NMR Ergebnissen.

Im fünften und letzten Kapitel wird ein besseres Verständnis des Orientierungsmechanismus von Proteinen erarbeitet und erste Ansätze für eine verbesserte Vorhersage der ladungsinduzierten Orientierung von Proteinen vorgestellt. Ausgehend von einer bekannten dreidimensionalen Struktur des Moleküls 
wird dazu ein verbessertes elektrostatisches Modell angewendet. Erste Ergebnisse deuten an, daß die Vorhersagekraft durch ein detailliertes elektrostatisches Modell gegenüber der des einfachen und in PALES implementierten Modells sich leicht verbessern könnte. 


\section{Acknowledgements}

Foremost, I would like to thank my supervisors Prof. Christian Griesinger and Dr. Markus Zweckstetter for providing me with the opportunity to complete my $\mathrm{PhD}$ thesis at the Max Planck Institute for Biophysical Chemistry, Göttingen, Germany. I am very grateful to Prof. Christian Griesinger for providing me with an excellent scientific environment, an outstanding infrastructure and also for his interest, encouragement and useful remarks. I especially want to thank my advisor on the spot, Dr. Markus Zweckstetter, whose support and guidance made my thesis work possible. He has been actively interested in my work and has always been available to advise me. I am very grateful for his patience, motivation, enthusiasm, and immense ideas and knowledge in NMR and structural biology that, taken together, make him a great mentor.

I would also like to thank Prof. Annette Zippelius for accepting me as her student in the Faculty of Physics, Georg-August Universität, Göttingen, Germany and Prof. Reiner Kree for their valuable advice and inputs. I would also like to thank other members of the thesis committee, Prof. Helmut Grubmüller, Prof. Tim Salditt and Dr. Marcus Müller for their support, advice and for evaluating my thesis.

I also take this opportunity to thank Nils-Alexander Lakomek for his generous help, Mrs. Sigrid Silberer and Dr. Jochen Junker for their help in various aspects through out my stay in the department. Much respect to my officemates and good friends, Hai-young Kim, Min-kyu Cho, Fernando Rodriguez-Castañeda for putting up with me for almost three years.

During this work I have interacted and benefited from many discussions with Dr. Young-Sang Jung, Dr. Monika Bayrhuber, Dr. Adam Lange, Dr. Vinesh Vijayan, Robert Schneider, Dr. Pierre Montaville, Dr. Karel Kubicek, Dr. Venkatesh Ramakrishnan, Dr. Dirk Bockelmann, Dr. Stefan Becker, Dr. Marc Baldus, Dr. Christophe Fares, Dr. Sigrun Rumpel, Dr. Peter Haberz, Marcel Reese, Marco Mukrasch, Dr. Edward d'Auvergne, for whom I have great regard, and I wish to extend my warmest thanks to all those who have helped me with my work in the Department of NMR based structural biology at Max Planck Institute for Biophysical Chemistry.

Additional crucial energy and vitality for this research was provided externally through my involvement in playing and promoting Cricket in Germany. Without which life wouldn't have been special and wouldn't have had opportunity to rub shoulders with great friends such as Saju Balakrishnan, Raghavendran Laxmi Narayanan, Rosan Ariyanayagam, Naeem Misdaq, Asad Ali Khan, Philip Brown, Alastor Premnath, Markus Nitsch and Dhushan Ekanayake.

Finally, I am forever indebted to my brother Devan and his family for their affection, love and constant support when it was most required. 


\section{Publication list:}

This thesis is based on the following papers:

Chapter 2

[1] M. Bayrhuber, V. Vijayan, M. Ferber, R. Graf, J. Korukottu, J. Imperial, J. E. Garrett, B. M. Olivera, H. Terlau, M. Zweckstetter, S.Becker. Conkunitzin-S1 is the first member of a new Kunitz-type neurotoxin family. Structural and functional characterization. Journal of Biological Chemistry, 2005, 25, 23766-70.

[2] J. Korukottu, M. Bayrhuber, P. Montaville, V. Vijayan, Y. S. Jung, S. Becker, M.Zweckstetter. Fast high-resolution protein structure determination by using unassigned NMR data. Angewandte Chemie International Edition English, 2007, 46, $1176-9$

Chapter 3

[3] J. Korukottu, A. Lange, V. Vijayan, R. Schneider, O. Pongs, S. Becker, M. Baldus, M. Zweckstetter. High-resolution 3D structure determination of Kaliotoxin by solid-state NMR spectroscopy. Submitted. 


\section{Table of contents}

$\begin{array}{lr}\text { Abbreviations } & 12\end{array}$

$\begin{array}{lr}\text { Chapter } 1 \text { General Introduction } & 15\end{array}$

1.1 Nuclear Magnetic Resonance (NMR) 15

1.1.1 The nuclear spin Hamiltonian $\quad 15$

1.1.2 Magnetization and pulses: 1D and multidimensional NMR 20

1.2 Solution NMR 22

1.2.1 Protein structure determination via solution NMR 22

1.2.2 Protein NMR experiments 23

$\begin{array}{ll}\text { 1.2.3 Assignment of resonances } & 27\end{array}$

1.2.4 Collection of structural restraints 28

1.2.4.1 Distance restraints 28

1.2.4.2 Dihedral angles restraints 30

1.2.4.3 Residual Dipolar couplings (RDCs) 32

1.2.5 Structure calculation via Simulated annealing $\quad 36$

$\begin{array}{ll}\text { 1.3 Solid-state NMR } & 42\end{array}$

1.3.1 Protein structure determination via solid-state NMR $\quad 45$

1.3.2 Collection of structural restraints 45

1.3.3 Structure calculation via simulated annealing $\quad 47$

Chapter 2 Fast High-resolution Protein Structure Determination by using $\begin{array}{ll}\text { Unassigned NMR data } & 48\end{array}$

$\begin{array}{ll}2.1 \text { Introduction } & 48\end{array}$

2.2 Material and Methods $\quad 53$

2.2.1 Input of unassigned NMR data 53

2.2.2 Backbone fold and assignment $\quad 56$

2.2.3 Side chain assignment and structure $\quad 56$ 
2.3 Results and Discussions

2.3.1 Backbone assignment and fold determination by FastNMR 63

2.3.2 Side chain assignment and assignment analysis 66

2.3.3 FastNMR produces correct high-resolution structure $\quad 67$

2.3.4 FastNMR tolerates missing and wrong backbone assignments

2.3.5 Influence of intermediate backbone conformation on convergence to a high-resolution structure

2.3.6 FastNMR tolerates spurious peaks and multiple conformation

Chapter 3 High-resolution 3D structure determination of kaliotoxin by solidstate NMR spectroscopy

3.1 Introduction

3.2 Materials and methods

3.3 Results and Discussions

3.3.1 High-resolution solid-state structure of KTX

3.3.2 Comparison of high-resolution solution-state NMR structure of KTX

3.3.3 Reliability of high-resolution solid-state structure of KTX

Chapter 4 High-resolution 3D structure of kaliotoxin bound to KesA-Kv1.3 and improved complex (KTX-KcsA-Kv1.3) structural model by solid-state NMR spectroscopy

4.1 Introduction 99

4.2 Materials and methods 101

4.2.1 Solid-state NMR data 101

4.2.2 Cross-peak assignment and structure calculation 
4.2.4 Docking of KTX with KcsA-Kv1.3 103

$\begin{array}{ll}\text { 4.3 Results and Discussions } & 105\end{array}$

4.3.1 Solid-state NMR structure of KTX in complex with KcsA-Kv1.3 105

4.3.2 Comparison with high-resolution solution-state and solid-state NMR structures of KTX

\section{Chapter 5 Understanding and Prediction of alignment and residual dipolar couplings of a protein from its known three-dimensional structure}

5.1 Introduction

5.1.1 Residual Dipolar Couplings (RDCs)

5.1.2 Electrostatics

5.2 Materials and methods

5.2.1 Experimental residual dipolar couplings

5.2.2 Coordinates and structural models

5.2.3 Model of protein/bilayer

5.2.4 Alignment tensor prediction

5.2.5 Electrostatic calculations

5.3 Results and Discussions 
$\begin{array}{lr}\text { Appendix } & 164\end{array}$

$\begin{array}{ll}\text { A Chemical shift assignment of KTX in solution } & 164\end{array}$

B Distance restraints for KTX in solid-state (free form) 167

C Chemical shift assignments of Conk-S2 170

D NMR restraints of Conk-S2 176

D.1 Distance constraints 176

$\begin{array}{ll}\text { D.2 Dihedral angles } & 187\end{array}$

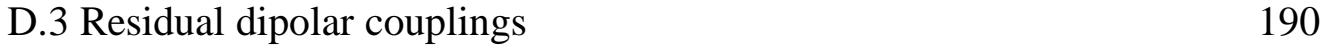

$\begin{array}{ll}\text { Curriculum Vitae } & 193\end{array}$

$\begin{array}{ll}\text { Lebenslauf } & 194\end{array}$ 


\section{Abbreviations:}

A

ADR

ARIA

B

BMRB

C

CANDID

$\mathrm{CBCA}(\mathrm{CO}) \mathrm{NH}$

Conk-S1

Conk-S2

COSY

CSA

CTX

D

DARR

DHPC

DMPC

DNA

DYANA

F

FastNMR

FDPB

FID ambiguous distance restraints

ambiguous restraints for iterative assignment

biological magnetic resonance bank

combined automated NOE assignment and structure

determination module

NMR experiment observing peptide ${ }^{15} \mathrm{~N},{ }^{1} \mathrm{H}^{N},{ }^{13} \mathrm{C}_{\alpha}$ and ${ }^{13} \mathrm{C}_{\beta}$

Conkunitzin-S1

Conkunitzin-S2

correlation spectroscopy

chemical shift anisotropy

charybdotoxin

dipolar assisted rotational resonance

dihexanoyl phosphatidylcholine

dimyristoyl phosphatidycholine

deoxyribonucleic acid

dynamics algorithm for NMR applications

\section{G}

fast structure determination by NMR

finite difference Poisson-Boltzmann

Free induction decay 
nuclear gyromagnetic ratio

H

h

Planck's constant

HNCA

HNCACB

NMR experiment observing peptide ${ }^{15} \mathrm{~N},{ }^{1} \mathrm{H}^{N}$, and ${ }^{13} \mathrm{C}_{\alpha}$

HNCO

NMR experiment observing peptide ${ }^{15} \mathrm{~N},{ }^{1} \mathrm{H}^{N},{ }^{13} \mathrm{C}_{\alpha}$ and ${ }^{13} \mathrm{C}_{\beta}$

NMR experiment observing peptide ${ }^{15} \mathrm{~N},{ }^{1} \mathrm{H}^{N}$, and previous ${ }^{13} \mathrm{CO}$

HN(CO)CA NMR experiment observing peptide ${ }^{15} \mathrm{~N},{ }^{1} \mathrm{H}^{N}$, and previous ${ }^{13} \mathrm{C}_{\alpha}$

HSQC heteronuclear single quantum correlation

I

IPAP inphase antiphase

$\mathbf{K}$

$\mathbf{K}^{+} \quad$ potassium

KNOWNOE automated assignment of NOESY NMR spectra using a knowledge based method

KTX Kaliotoxin

$\mathbf{L}$

M

MARS automatic backbone resonance assignment program

MAS magic angle spinning

$\mu_{0} \quad$ magnetic permeability of vacuum

$\mathbf{N}$

NMR

nuclear magnetic resonance

NOAH

automatic program to assign NOESY cross-peaks

NOE

nuclear Overhauser effect 
PALES

PASD

PC

PDB

PDSD

Pf1

PS

$\mathbf{R}$

RDC

RF

RMSD

RNA

$\mathbf{S}$

SA

SSNMR

SVD

$\mathbf{T}$

$\mathbf{T}_{1}$

$\mathbf{T}_{2}$

TALOS

TOCSY

TROSY prediction of alignment from structure

probabilistic assignment algorithm for automated structure

determination

phosphatidylcholine

protein data bank

proton driven spin diffusion

filamentous bacteriophage

phosphatidylserine

residual dipolar coupling

radio frequency

root mean square deviation

ribonucleic acid

simulated annealing

solid-state NMR

singular value decomposition

longitudinal or spin-lattice relaxation time

transversal or spin-spin relaxation time

torsion angle likelihood obtained from shifts and sequence

similarity

total correlation spectroscopy

transverse relaxation optimized spectroscopy 


\section{Chapter 1 General Introduction}

\subsection{Nuclear Magnetic Resonance (NMR)}

\subsubsection{The nuclear spin Hamiltonian}

NMR is a technique for investigating the chemical and spatial structure of compounds, by exploiting a property, that some nuclei have, called spin. The nuclear spin is a quantum effect, and the associated quantum numbers I are multiple of 1/2. The number of quantum states is $2 I+1$. In a simplified model, we can imagine the nucleus with spin $1 / 2$ spinning around an axis with only two possible orientations, up and down. Since it is a moving charge, the spinning nucleus generates a magnetic field. When there is no external magnetic field present, there is no energy difference between up and down spin states. If an external magnetic field $\mathrm{B}_{0}$ is applied, then the spin state that gives rise to a nuclear magnetic field aligned to the external magnetic field has a different, lower, energy from the other spin state. The energy difference $\Delta \mathrm{E}$ is small and falls in the range of radio frequencies (Figure 1.1).

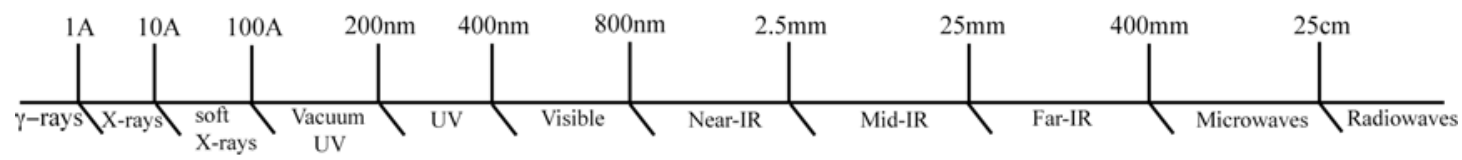

Figure 1.1: Range of frequencies exploited by different spectroscopies to investigate the matter

The interaction between a nuclear spin $\mathbf{I}$ and a static magnetic field $\mathbf{B}_{\mathbf{0}}$ is called Zeeman interactions and can be described by a second-rank tensor ( $3 \times 3$ matrix) $\bar{Z}$ :

$$
\mathrm{H}_{\text {zeeman }}=\mathbf{I} \widehat{Z}_{\mathbf{B}_{0}}
$$


The energy separation between two different Zeeman states (Larmor frequency) is given by:

$$
\Delta E=\hbar v B_{0}
$$

and is directly proportional to the static magnetic field applied, where $\hbar$ is the Planck constant and $v$ is the frequency. The population differs for the two states and the ratio is given by Boltzmann distribution. The lowest-energy state, corresponding to a parallel orientation of nuclear spins along the magnetic field, is slightly more populated. As a result, the vector sum of all nuclear spins originates a macroscopic magnetic moment, named magnetization.

By irradiation with a suitable energy, it is possible to convert a nucleus from one spin state to the other. Such irradiations with radiofrequencies of short duration are usually named pulses.

The energy interaction between the spin I and the RF pulse is, analogously:

$$
\mathrm{H}_{\mathrm{rf}}=\mathbf{I} \hat{\mathbf{Z}} \mathbf{B}_{\mathbf{I}}
$$

The power of NMR spectroscopy relies on the fact that nuclei in different parts of the molecule experience different local magnetic fields according to the molecule's structure, and consequently resonate (adsorb energy) at different frequencies. It means that similar atoms in different environments, such as carbon-bonded or oxygen-bonded hydrogen atoms, show different frequencies. This effect of the chemical environment on the absorbed frequency $v$ is called chemical shift. By defining a reference frequency $v_{0}$, it is possible to express chemical shifts as dimensionless numbers, such that the value is not a function of the external magnetic field $\mathrm{B}_{0}$, which allows direct comparison of spectra from NMR spectrometers with different magnetic field strengths and operating frequencies: $\delta=\frac{v-v_{0}}{v_{0}}$. Chemical 
shifts are a potent means of identifying different nuclei within the protein and discriminating between them. These effects of the environment on the nuclear spin depend on the orientation of the sample with respect to the magnetic field and are therefore described by a second-rank chemical shift (or shielding) tensor $\hat{\Sigma}$ :

$$
\operatorname{Hcs}=\boldsymbol{\gamma}\left(\hat{\Sigma} \mathbf{B}_{0}\right)
$$

where $\gamma$ is the gyromagnetic ratio of the spin $\mathbf{I}$ and $\left(\hat{\Sigma} \mathbf{B}_{0}\right)$ represents the effective magnetic field experienced locally by the spin $\mathbf{I}$.

This dependence of the chemical shift on the orientation is referred to as chemical shift anisotropy (CSA). Generally, it is possible to express the chemical shift tensor in a coordinate system, so that all off-diagonal elements vanish. In this principal axes system, the chemical shift tensor is fully described by the three diagonal elements the principal components $\left(\delta_{11}, \delta_{22}\right.$ and $\left.\delta_{33}\right)$ - and the three eigenvectors or Euler angles describing the orientation of the principal axes with respect to an arbitrary frame.

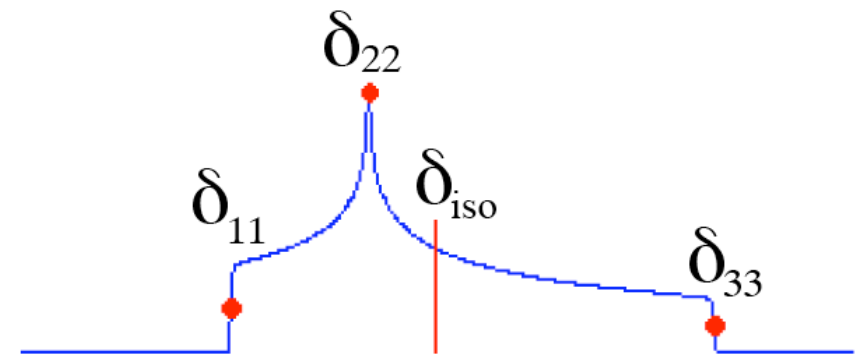

Figure 1.2: NMR Absorption line under anisotropic conditions for a powder.

Thus, $\delta_{11}$ corresponds to the direction of least shielding, with the highest frequency, while $\delta_{33}$ corresponds to the direction of highest shielding, with the lowest frequency:

$$
\delta_{11} \geq \delta_{22} \geq \delta_{33}
$$

The isotropic values, $\delta_{\text {iso, }}$, are the average values of the principal components, and 
correspond to the centre of gravity of the line shape:

$$
\delta_{i s o}=\frac{\left(\delta_{11}+\delta_{22}+\delta_{33}\right)}{3}
$$

Under isotropic conditions (in solution, or, in the solid state, upon rapid sample spinning $(\S 1.3)$ ), an absorption line in correspondence to $\delta_{\text {iso }}$ will be observed and the Hamiltonian term simplifies into:

$$
\operatorname{Hcs}=\boldsymbol{\gamma}\left(\sigma_{\text {iso }} \mathbf{B}_{\mathbf{0}}\right)
$$

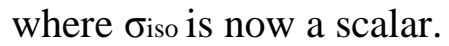

Nuclear spins within a molecule may interact with each other. Several interactions of different nature are usually simultaneously active. The scalar coupling (or $J$ coupling) represents the interaction between nuclei relayed through the electrons in the chemical bonds. The strength of the scalar interaction between two spins $\mathbf{I}$ and $\mathbf{S}$ is represented by a coupling constant $J$ :

$$
\mathrm{H}_{\boldsymbol{J}}=\mathbf{I} \mathbf{J} \mathbf{S}
$$

Homonuclear (proton) couplings between two atoms distant by two bonds are largest $(\sim 15 \mathrm{~Hz})$, smaller for three-bond couplings $(5-10 \mathrm{~Hz})$, and smallest for long-range couplings $(\sim 1 \mathrm{~Hz})$. Heteronuclear coupling are substantially larger. For example, proton-nitrogen couplings are around $90 \mathrm{~Hz}$ and proton-carbon couplings are on the order of $140 \mathrm{~Hz}$.

A second interaction between nuclear spins that takes place is the dipolar coupling. Nuclear spins behave indeed as magnetic moments, and consequently interact with each other through space. The dipolar interaction is orientation-dependent and is therefore represented by a second-rank dipolar tensor $\hat{D}$ :

$$
\mathrm{HD}_{\mathrm{D}}=\mathbf{I} \hat{\mathrm{D}} \mathbf{S}
$$

The dipolar tensor is a traceless tensor, that is, the sum of its diagonal elements is zero. Therefore, unlike the chemical shift, dipolar interactions are averaged to zero in 
solution due to the isotropic tumbling of molecules $(\S 1.2 .4 .3$ Residual Dipolar Coupling) or in the solid-state under efficient sample spinning at the magic angle. However, the magnetic field that is generated from the dipolar coupling can have large effects on the spin-lattice relaxation rates of the spins $(\S 1.2 .4 .1$, Nuclear Overhauser effect).

In short, it is common to classify the NMR interactions as a total spin Hamiltonian,

$$
\hat{H}=\hat{H}_{z e e m a n}+\hat{H}_{r f}+\hat{H}_{C S}+\hat{H}_{J}+\hat{H}_{D}+\hat{H}_{Q}
$$

which is simply a sum of all the terms described above. For spins $I=1 / 2$ quadrupolar interaction $\hat{H}_{Q}$ applies only for nuclei with spins larger than $1 / 2$ and is therefore not considered further in this thesis.

Compared to other spectroscopy, a major advantage with NMR is the possibilities to modify at will the Hamiltonian, with few restrictions, adapting it to the special requirements of the problem to be solved. The ease with which the nuclear spin Hamiltonian can be modified depends on the fact that the nuclear interactions are very weak compared to the interactions exploited in other spectroscopic techniques, such as infrared spectroscopy or VIS-UV spectroscopy. In order to override an interaction, an alternative, competitive perturbation of the system has to be applied. To be effective, the energy of the perturbation has to be significantly larger than the interaction to manipulate. Examples of this are spin decoupling and sample spinning, vital tools in solution and solid-state NMR.

In general, by playing with the energy terms of the Hamiltonian, it is possible to design a large number of NMR experiments, which provide different information: e.g., it is possible to obtain spectra which establish through-bond correlations between nuclei showing scalar coupling, or rather through-space correlations between nuclei 
showing dipolar coupling.

\subsubsection{Magnetization and pulses: 1D and multidimensional NMR}

Upon application of appropriate pulses, rotations of the magnetization can be induced.

A major property of the magnetization is that its component in the plane perpendicular to the axis of the external magnetic field rotates around this axis with an angular velocity proportional to the Larmor frequency, that is the frequency $v$ of electromagnetic radiation required to excite transitions between Zeeman levels (Equation 1.2). If we now introduce a frame that rotates with this frequency, the magnetization appears static. However, spins are also evolving under their chemical shift (Equation 1.7), which depends on the gyromagnetic ratio $(\gamma)$ and the shielding by the chemical environment. The precession of the several components of the magnetization along the static magnetic field constitutes a time-varying magnetic field, which in turn has the property to induce an electromotive force (according to Maxwell it is an electrical field) in a coil appropriately located close to the sample.

The measured intensity of the generated alternating current as a function of time is called FID (Free Induction Decay). In the case of a protein, the bulk magnetic moment is originated by nuclear spins that have in general different chemical shifts. Hence, the FID contains all different resonance frequencies of the nuclei, corresponding to their chemical shifts. By Fourier transformation ${ }^{\mathrm{a}}$, the FID can be converted into a frequency function, that is, the NMR spectrum. To study biopolymers like proteins, DNA and RNA, 1D NMR spectroscopy is unable to resolve the frequency of the individual nuclear spins. Hence, additional spectral dimensions were introduced, like, 2D, 3D and even 4D spectroscopic techniques, to

\footnotetext{
${ }^{\mathrm{a}}$ Fourier transformation is an important mathematical operation, which allows transforming time domain data into the frequency domain and vice versa.
} 
increase resolution and to extract additional information. The invention of multidimensional spectra was a major leap in NMR spectroscopy. Every 2D experiment, for example, can be described with a simple basic scheme, consisting of a preparation period, an evolution period t1 (during which the spins are labeled according to their chemical shift), a mixing period where spins are correlated to each other, and finally a detection period. For measuring a 2D spectrum, many FIDs are acquired for incremented values of the $\mathrm{t}_{1}$ delay (evolution period) to generate a second frequency dimension. The recorded FIDs are then Fourier transformed with respect to both $t_{1}$ and $t_{2}$ (as illustrated in Figure 1.3). Signal with two different frequency coordinates (cross-peaks) indicate a correlation between two nuclei. The length of the mixing period is commonly referred to as mixing time. The intensity of each crosspeak as a function of the length of the mixing time represents the build-up curve for the cross-peak.

\section{Two Dimensional NMR}

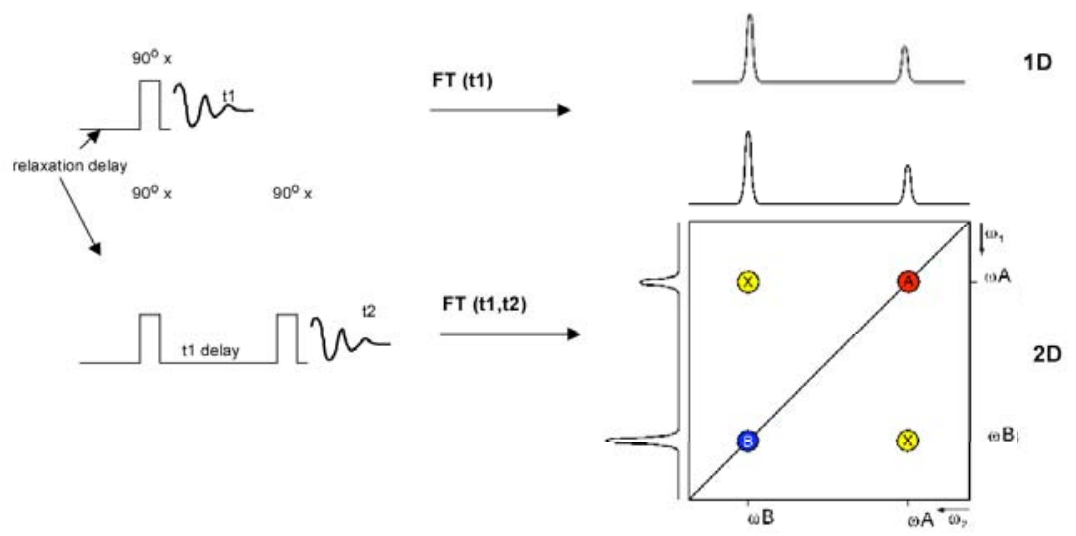

Figure 1.3: Principles of 2D NMR spectroscopy.

In $3 \mathrm{D}$ or $4 \mathrm{D}$ spectra, the $2 \mathrm{D}$ peaks are dispersed along one or two more orthogonal axes using the chemical shifts of one or two bonded heteronuclei. 


\subsection{Solution NMR}

Over the last two decades, solution NMR has become a major technique in structural biology. It has proven to be a powerful technique to investigate protein structures in solution at atomic definition. Compared to X-ray crystallography, solution NMR allows not only to investigate the structures of biopolymers in a nearly physiological environment, but also to determine their dynamic properties. Hence, solution NMR is a vital tool also in protein biophysics, allowing for the study of protein-ligand and protein-protein interactions, protein folding, kinetics and catalysis.

\subsubsection{Protein structure determination via solution NMR}

Proteins are an important class of biological macromolecules present in all biological organisms, made up of elements such as carbon, hydrogen, nitrogen, oxygen and sulfur. Proteins are biopolymers of combination of 20 amino acids, characterized by four structural levels as shown in Figure 1.4: (1) primary structure given by amino acid sequence, (2) secondary structure defined by the local conformation of the

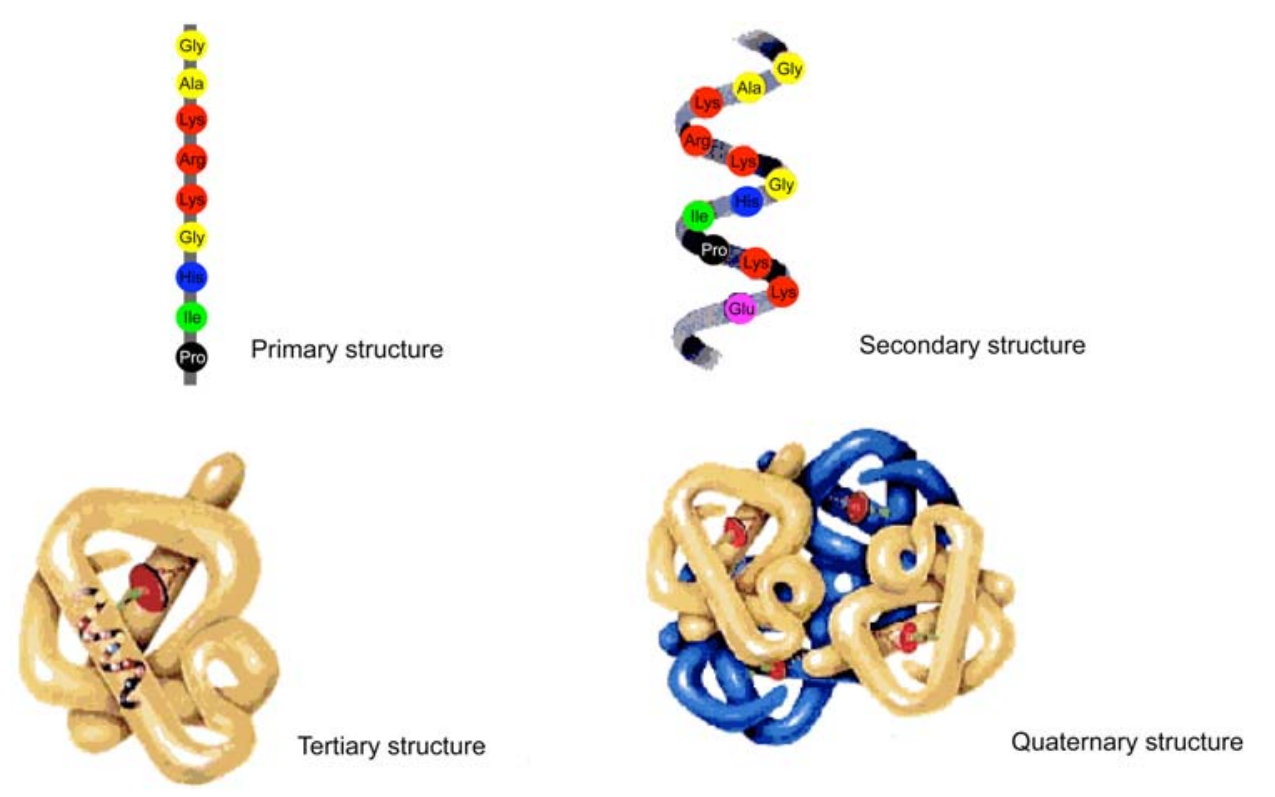

Figure 1.4: Structural levels of proteins: primary, secondary, tertiary and quaternary 
backbone, (3) tertiary structure represented by the spatial proximity of the secondary structural elements and (4) quaternary structure that specifies the packing of several polypeptide chains. To be able to perform their biological function, protein folds into one, or more, specific spatial conformations, driven by number of noncovalent interactions such as hydrogen bonding, ionic interactions, Van der Waals' forces and hydrophobic packing.

In order to understand the functions of proteins at a molecular level, it is often necessary to determine the three dimensional structure of proteins. This is the topic of the scientific field of structural biology, that employs techniques such as X-ray crystallography and NMR spectroscopy, to determine the structure of proteins. There are also many $a b$ initio methods which predict the protein structure from the primary sequence based on similarity and comparison. The commonly employed procedure for structure determination of proteins based on solution NMR consists of several consecutive steps, covering sample preparation, NMR data acquisition, peak-picking, resonance assignment, collection of distance- and additional structural restraints (such as Residual dipolar couplings, hydrogen bonds, disulphide bridges) and, finally, structure calculation and structure refinement (shown in Figure 1.5) [Wuthrich 1986]. Till date, this is the most widely followed strategy for determination of protein structures in solution, despite several alternative promising approaches that mostly aim at omission of the cumbersome and biologically irrelevant resonance-assignment [Grishaev and Llinas 2002a].

\subsubsection{Protein NMR experiments}

There are three $2 \mathrm{D}$ spectra that are widely used for the structure determination of proteins with a mass of up to 10kD: 2D COSY, 2D TOCSY and 2D NOESY 
[Wuthrich, Wider 1982] are the most commonly used homonuclear (signals of the same isotope (usually ${ }^{1} \mathrm{H}$ ) are detected during the evolution periods) correlation experiments exploiting $\mathbf{J}$ (scalar) couplings. Apart from protons a protein contains other magnetic active nuclei. For NMR of proteins, ${ }^{15} \mathrm{~N}$ and ${ }^{13} \mathrm{C}$ are of special importance. The use of these hetero nuclei allows some new features in NMR, which facilitate the structure determination especially of larger proteins (> 100 amino acids).

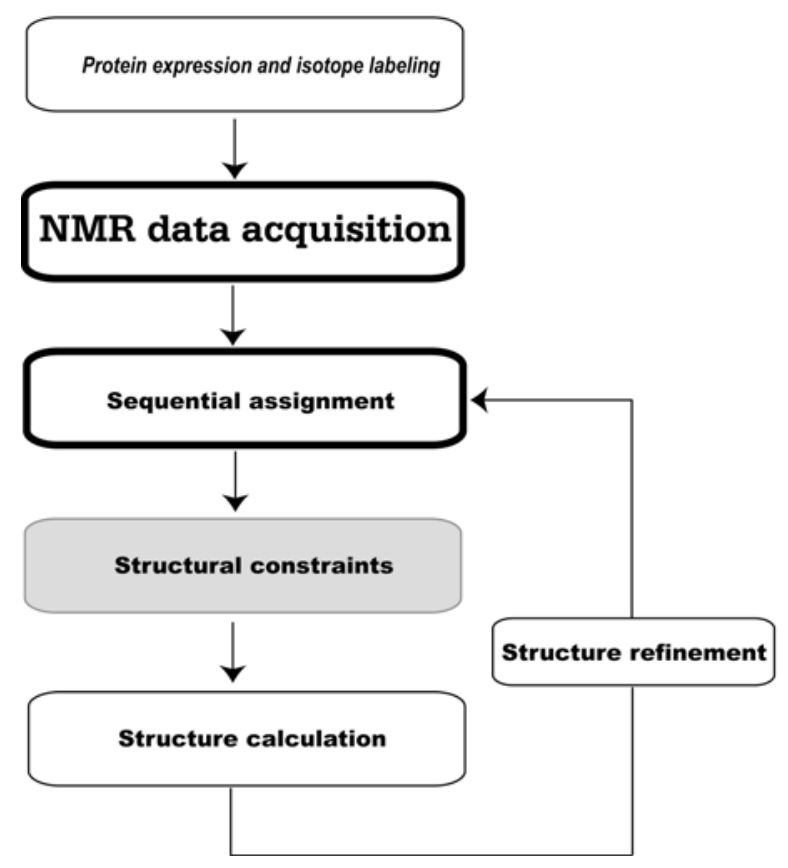

Figure 1.5: Strategy of NMR protein structure determination.

The most important heteronuclear experiment is HSQC (Heteronuclear Single Quantum Coherence) (Figure 1.6). Each signal in a HSQC spectrum represents a proton that is bound to a nitrogen atom. The spectrum contains the signals of the $\mathrm{H}^{\mathrm{N}}$ protons in the protein backbone. Since there is only one backbone ${ }^{1} \mathrm{H}^{\mathrm{N}}$ per amino acid, each HSQC signal represents on single amino acid.

2D spectra of proteins are often crowded with signals. Therefore, these spectra are spread out in a third dimension, so that signals are distributed in a cube instead of a plane. This spread out is achieved by combining HSQC and NOESY in a single 3D 
experiment: The NOESY is extended by an HSQC step. The resulting experiment is called 3D NOESY-HSQC. In a similar way, a TOCSY-HSQC can be constructed by combining the TOCSY and the HSQC experiment.

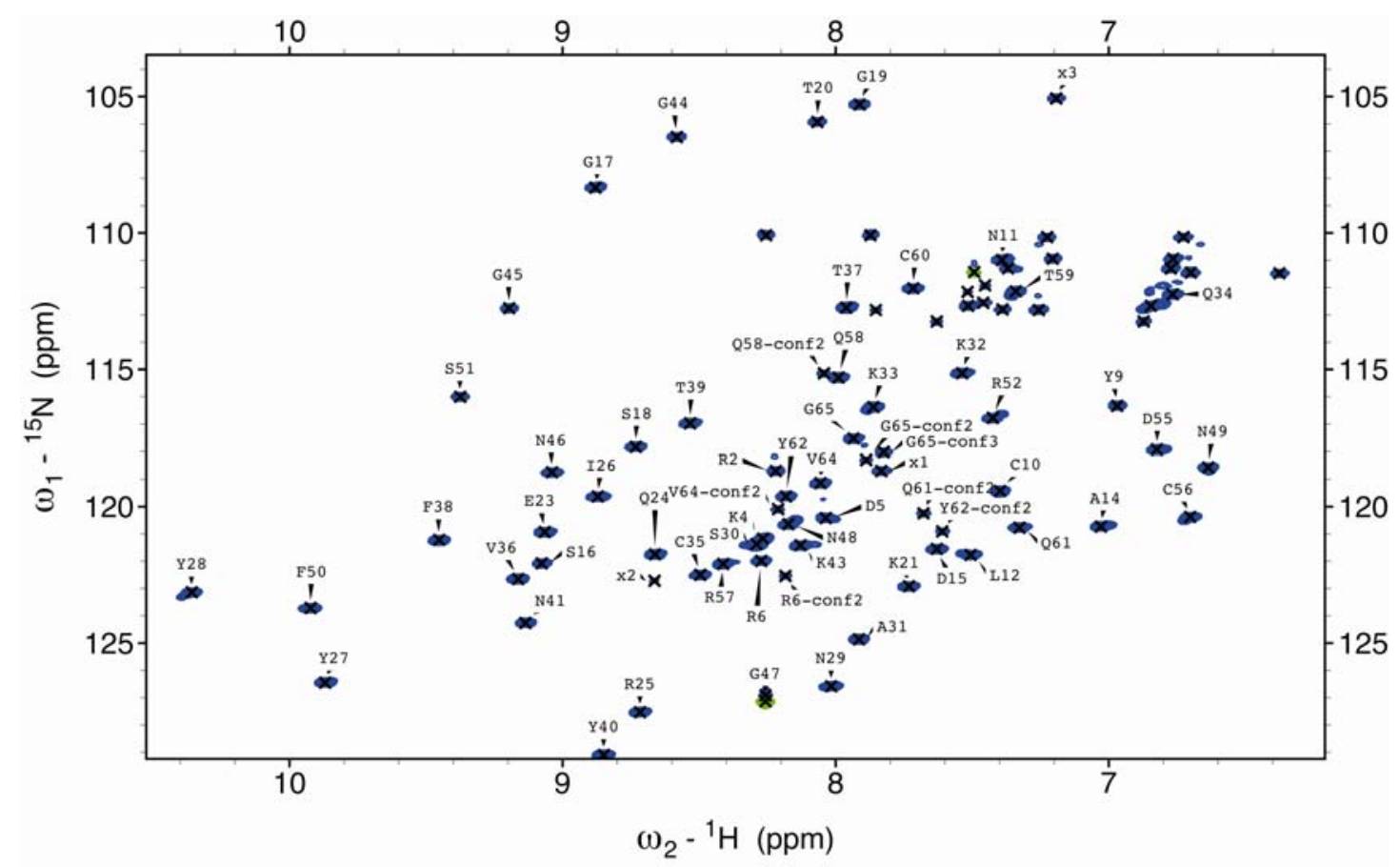

Figure 1.6: $2 \mathrm{D}{ }^{1} \mathrm{H}^{-}{ }^{15} \mathrm{~N}$ HSQC of Conkunitzin-S2 with some assignments marked

Triple resonance experiments are the method of choice for the sequential assignment of proteins. These experiments are called 'triple resonance' because three different nuclei $\left({ }^{1} \mathrm{H},{ }^{15} \mathrm{~N}\right.$ and $\left.{ }^{13} \mathrm{C}\right)$ are correlated. The experiments are performed on doubly labeled $\left({ }^{13} \mathrm{C},{ }^{15} \mathrm{~N}\right)$ proteins. The names of the triple resonance experiments are very descriptive. The names of all nuclei, which are used for magnetization transfer during the experiment, are listed in the order of their use, bracketing the names of nuclei which are used only for transfer and whose frequencies are not detected. HNCACB, $\mathrm{CBCA}(\mathrm{CO}) \mathrm{NH}$ are the two key experiments to establish correlations along the protein backbone via heteronuclear correlations. 


\section{HNCACB}

The HNCACB experiment correlates ${ }^{13} \mathrm{C}_{\alpha}$ and ${ }^{13} \mathrm{C}_{\beta}$ resonances of an amino acid with the ${ }^{1} \mathrm{H}^{\mathrm{N}}$ and ${ }^{15} \mathrm{~N}$ resonances of the same residue and ${ }^{1} \mathrm{H}^{\mathrm{N}}$ and ${ }^{15} \mathrm{~N}$ resonances of the neighboring residue (Figure 1.7). In this experiment $\mathrm{N}(\mathrm{i}), \mathrm{HN}(\mathrm{i}), \mathrm{C}_{\alpha}(\mathrm{i})$ and $\mathrm{C}_{\beta}(\mathrm{i})$

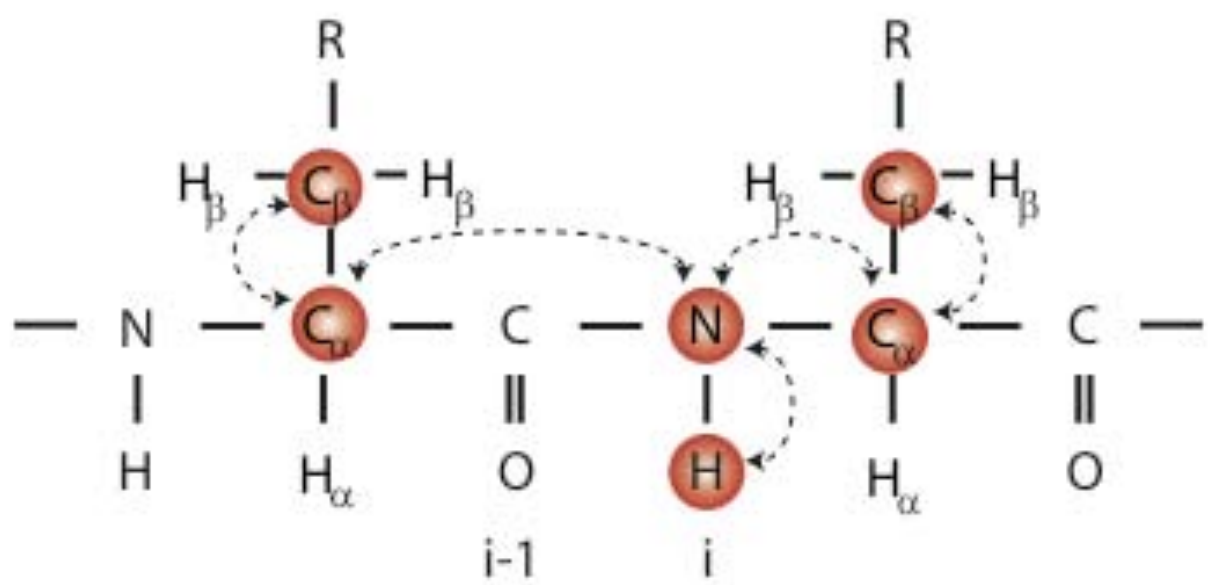

Figure 1.7: HNCACB experiment: The magnetization is transferred from the ${ }^{1} \mathrm{H}^{\mathrm{N}}(\mathrm{i})$ $\rightarrow{ }^{15} \mathrm{~N}(\mathrm{i}) \rightarrow{ }^{13} \mathrm{C}_{\alpha}(\mathrm{i}) /{ }^{13} \mathrm{C}_{\alpha}(\mathrm{i}-1)$ and then from there to ${ }^{13} \mathrm{C}_{\beta}(\mathrm{i}) /{ }^{13} \mathrm{C}_{\beta}(\mathrm{i}-1)$. After which it comes back to ${ }^{1} \mathrm{H}^{\mathrm{N}}$ (i) along the same path. The frequencies of ${ }^{1} \mathrm{H}^{\mathrm{N}}(\mathrm{i}),{ }^{15} \mathrm{~N}(\mathrm{i}),{ }^{13} \mathrm{C}_{\alpha}(\mathrm{i})$, ${ }^{13} \mathrm{C}_{\mathrm{B}}(\mathrm{i}),{ }^{13} \mathrm{C}_{\alpha}(\mathrm{i}-1)$ and ${ }^{13} \mathrm{C}_{\mathrm{B}}(\mathrm{i}-1)$ (red) are observed.

resonances of the $\mathrm{i}^{\text {th }}$ amino acid and ${ }^{13} \mathrm{C}_{\alpha}(\mathrm{i}-1)$ and ${ }^{13} \mathrm{C}_{\beta}(\mathrm{i}-1)$ resonances of the preceding amino acid are observed. For a medium-sized protein $(\sim 15 \mathrm{KDa})$, this experiment alone can provide virtually complete sequential assignment of the ${ }^{1} \mathrm{H}_{\mathrm{N}}$, ${ }^{15} \mathrm{~N},{ }^{13} \mathrm{C}_{\alpha}$ and ${ }^{13} \mathrm{C}_{\beta}$ resonances, because in addition to the sequential connectivities, the ${ }^{13} \mathrm{C}_{\alpha}$ and ${ }^{13} \mathrm{C}_{\beta}$ chemical shifts provide information on the amino acid type.

\section{CBCA(CO)NH}

The $\mathrm{CBCA}(\mathrm{CO}) \mathrm{NH}$ experiment correlates both the ${ }^{13} \mathrm{C}_{\alpha}$ and ${ }^{13} \mathrm{C}_{\beta}$ resonances of an amino acid residue with the ${ }^{1} \mathrm{H}^{\mathrm{N}}$ and ${ }^{15} \mathrm{~N}$ resonances of the preceding residue (Figure 1.8). In this experiment, ${ }^{13} \mathrm{C}_{\beta}(\mathrm{i}-1),{ }^{13} \mathrm{C}_{\alpha}(\mathrm{i}-1),{ }^{15} \mathrm{~N}(\mathrm{i})$ and ${ }^{1} \mathrm{H}_{\mathrm{N}}(\mathrm{i})$ resonances are 
observed. This experiment is useful to circumvent the degeneracy between the intraresidue $\left({ }^{13} \mathrm{C}_{\beta}(\mathrm{i})\right.$ and $\left.{ }^{13} \mathrm{C}_{\alpha}(\mathrm{i})\right)$ and inter-residue $\left({ }^{13} \mathrm{C}_{\beta}(\mathrm{i}-1)\right.$ and $\left.{ }^{13} \mathrm{C}_{\alpha}(\mathrm{i}-1)\right)$ chemical shifts.

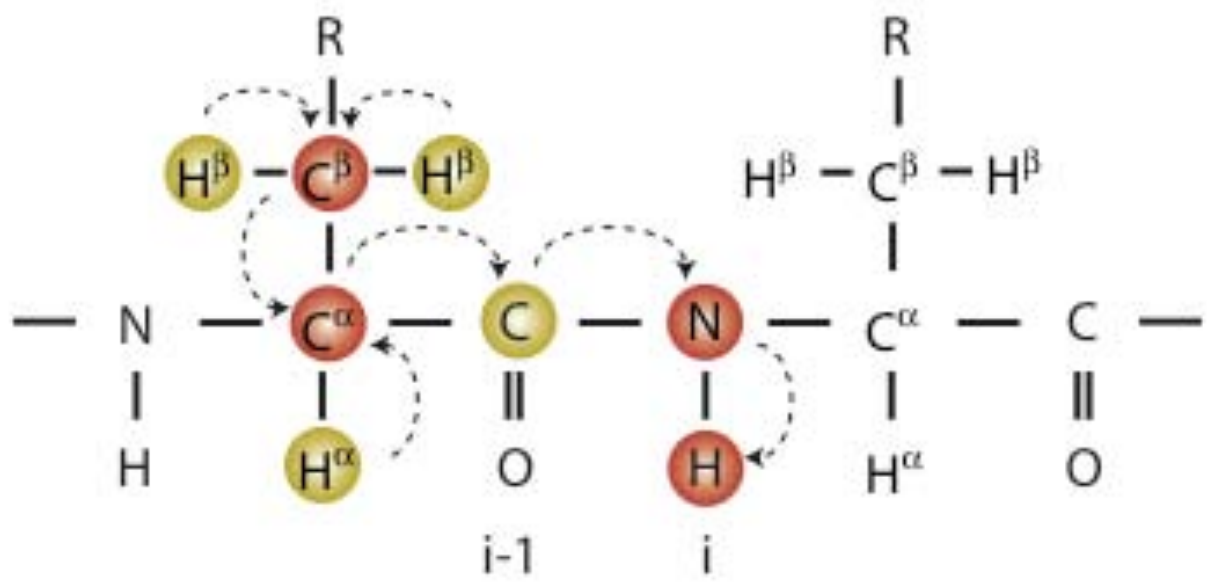

Figure 1.8: $\mathrm{CBCA}(\mathrm{CO}) \mathrm{NH}$ experiment: The magnetization is transferred from the ${ }^{1} \mathrm{H}_{\alpha}(\mathrm{i}-1) / 1 \mathrm{H}_{\beta}(\mathrm{i}-1) \rightarrow{ }^{13} \mathrm{C}_{\alpha}(\mathrm{i}-1) /{ }^{13} \mathrm{C}_{\beta}(\mathrm{i}-1) \rightarrow{ }^{13} \mathrm{C}^{\prime}(\mathrm{i}-1) \rightarrow{ }^{15} \mathrm{~N}(\mathrm{i}) \rightarrow{ }^{1} \mathrm{H}^{\mathrm{N}}(\mathrm{i})$. The ${ }^{1} \mathrm{H}_{\alpha}$, $1 \mathrm{H}_{\beta}$ and ${ }^{13} \mathrm{C}^{\prime}$ (yellow) act only as relay nucleus, their frequency are not detected. The frequencies of ${ }^{1} \mathrm{H}^{\mathrm{N}}(\mathrm{i}),{ }^{15} \mathrm{~N}(\mathrm{i}),{ }^{13} \mathrm{C}_{\alpha}(\mathrm{i}-1)$ and ${ }^{13} \mathrm{C}_{\beta}(\mathrm{i}-1)$ (red) are observed.

\subsubsection{Assignment of resonances}

The aim of the analysis of NMR spectra is to extract all available structural information of the proteins, such as, inter-atomic distances, torsion angles etc. A plethora of $2 \mathrm{D}$ and $3 \mathrm{D}$ experiments is present in the literature designed for assigning each observed chemical shift to a nuclear spin, for instance, ${ }^{1} \mathrm{H},{ }^{15} \mathrm{~N}$ and ${ }^{13} \mathrm{C}$ etc., within the protein. This procedure is referred to as resonance assignment. The assignment of the resonances is usually done in two different separate steps. First, resonances within each single amino acid are assigned. As a result, chemical shifts are grouped in different spin systems, one for each amino acid. Second, the different spin systems are connected with each other by exploiting scalar couplings between heteronuclei in the backbone, or, alternatively, through-space correlations between protons [Wuthrich 1986].

From the combination of $\mathrm{CBCA}(\mathrm{CO}(\mathrm{NH}$ and $\mathrm{HNCACB}$ experiments backbone 
resonance and the sequential connectivities are obtained. These experiments provide the ${ }^{13} \mathrm{C}_{\alpha}$ and ${ }^{13} \mathrm{C}_{\beta}$ chemical shifts to establish the sequential link between neighboring residues. Furthermore, when both ${ }^{13} \mathrm{C}_{\alpha}$ and ${ }^{13} \mathrm{C}_{\beta}$ chemical shifts are provided at the same time, it gives important information about the amino acid type and secondary structure (e.g. $\alpha$-Helix and $\beta$-strand). However, the quality of the spectra frequently makes the assignment process difficult, because of the ambiguity of sequential connections, missing chemical shifts, additional artifact peaks and isolated connectivities due to either missing chemical shifts or the occurrence of prolines in the amino acid sequences, which are not observable due to lack of the ${ }^{1} \mathrm{H}^{\mathrm{N}}$ atom. Assigning the resonances is the critical step in the strategy of structure determination, due to limited resolution and spectral overlap. In the end, the quality of the determined structures depends on the number of correct assignments.

\subsubsection{Collection of structural restraints}

In protein NMR spectroscopy, structure calculations are usually carried out using, (1) distance between two specific atoms (NOE) obtained from multidimensional NOESY spectra, (2) dihedral angle constraints derived from chemical shifts and $\mathbf{J}$ coupling constants. (3) residual dipolar couplings (RDCs). In some cases, disulphide and/or hydrogen bond distance constraints from other experimental data are also included.

\subsubsection{Distance restraints}

In solution NMR, molecular tumbling produces an isotropic Hamiltonian, were only isotropic chemical shifts and J couplings are left. The dipolar coupling vanishes in solution; nevertheless it affects nuclear spin relaxation. In particular, the relaxation of a spin is influenced via dipolar coupling by the presence of another close spin, whose 
spin population has been perturbed. These effects on spin relaxation are called Nuclear Overhauser effect (NOE) [Jeener, Meier 1979;Macura and Ernst 1980;Solomon 1955], and have the important property of depending on internuclear distances, hence they provide a way to measure them. NOESY is the $2 \mathrm{D} / 3 \mathrm{D}$ solution NMR experiment, which directly exploits this effect to correlate nuclei that are close in space (distance smaller than $5 \AA$ ). NOE is by far the most important NMRobservable used in determining protein structures. While chemical shifts and Jcoupling constants give local structural information, NOE data can relate atoms that are far apart in the series of chemical bonds connecting the biomolecule, but are close in space.

Using a first-order approximation, the NOE cross-peak intensities or volume $\mathrm{V}$ is proportional to the relaxation rates and may be expressed as [Jeener, Meier 1979]:

$$
\mathrm{V}=\left\langle\mathrm{r}_{\mathrm{ij}}^{-6}\right\rangle \mathrm{f}\left(\tau_{\mathrm{c}}\right)
$$

It depends on the distance between the two nuclei $\mathrm{i}$ and $\mathrm{j}, \mathrm{r}_{\mathrm{ij}}$, as well as on the rotational correlation time $\tau_{\mathrm{c}}$ (describing the Brownian tumbling motion of the protein in solution). By measuring cross-peak intensities $\mathrm{V}$, distance restraints can be derived. It can be estimated in the 2D NOESY, 3D ${ }^{15} \mathrm{~N}$-NOESY HSQC and $3 \mathrm{D}{ }^{13} \mathrm{C}$ NOESY-HSQC spectra.

Distances are derived from the spectra after calibration against NOE signals for known distances (such as distances in elements of secondary structure elements) and grouped into few classes. An upper and lower bound of distance is assigned to each class. The lower bound is often set to the sum of the van der Waals radii of the two protons. In this procedure, all non-sequential signals that are visible in the NOESY spectra have to be assigned, the number of which easily exceeds 1000 in a medium sized protein (ca. 120 amino acids). It is distinguished between cross peaks of protons 


\section{NOE class Distance $[\AA] \quad$ Upper bound $[\AA]$}

$\begin{array}{ccc}\text { very strong } & 2.3 & 2.5 \\ \text { strong } & 2.8 & 3.1 \\ \text { medium } & 3.1 & 3.4 \\ \text { weak } & 3.5 & 3.9 \\ \text { very weak } & 4.2 & 5.0\end{array}$

Table 1.1: NOE distance bounds used in protein structure determination.

no more than five amino acids apart in the protein sequence (medium range NOE's) and those, which are more than five amino acids apart (long range NOE's). The former are mainly indicative of the protein backbone conformation and are used for secondary structure determination, whereas the latter are an expression of the global structure of the protein and therefore contain the main information used for tertiary structure calculation. NOE assignment is one of the most time consuming and difficult parts for the structure determination due to the ambiguity of the peaks and overlaps in the NOESY spectra.

\subsubsection{Dihedral angle restraints}

Dihedral angle restraints represent a second important group of restraints that is possible to derive from NMR spectra. Dihedral angles in the protein backbone influence directly the three-bond scalar couplings ${ }^{3} J$ constants via Karplus equation [Karplus 1963]:

$$
{ }^{3} J(\tau)=A \cos ^{2}(\tau)+B \cos (\tau)+C
$$

The constants A, B and C depends on the particular nuclei involved in the covalent 


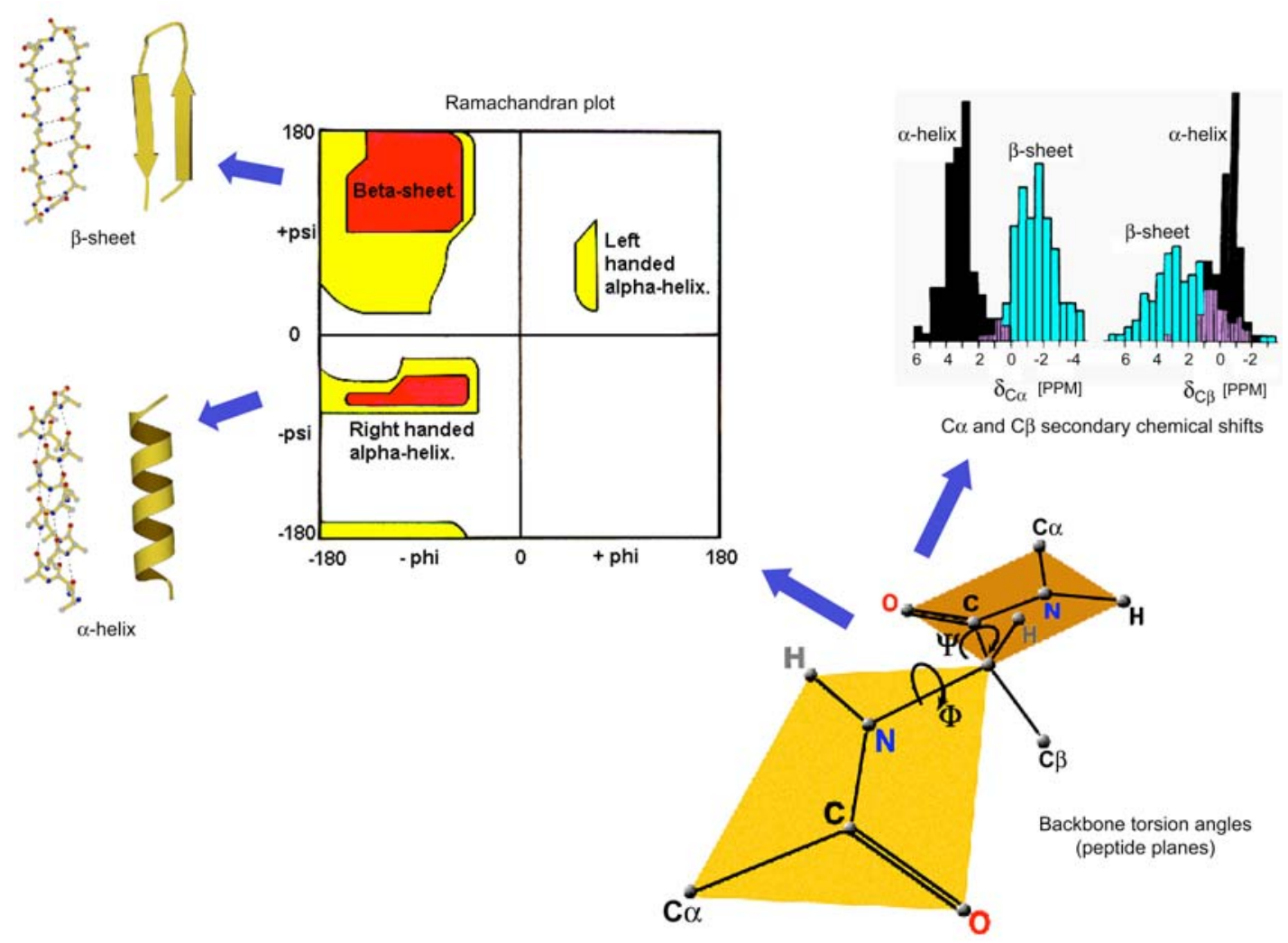

Figure 1.9: Elements of protein secondary structure ( $\alpha$-helix and $\beta$-sheet) defined by the backbone dihedral angles $\left(\phi\right.$ and $\psi$ ) and empirical correlations with ${ }^{13} \mathrm{C}_{\alpha}$ and ${ }^{13} \mathrm{C}_{\beta}$ secondary chemical shifts [Cornilescu, Delaglio 1999; Wishart 1994].

bonds and change depending on which dihedral angle $\left(\varphi, \psi\right.$ or $\left.\chi_{1}\right)$ the letter $\tau$ stands for. Traditionally, $\varphi$ angles are obtained by measurement of ${ }^{3} J_{H^{\alpha} H^{N}}$ constants, whereby $\chi_{1}$ angles from ${ }^{3} J_{H^{\alpha} H^{\beta}}$ constants. Hence, after measuring $\mathrm{J}$ couplings, it is possible to restrain the backbone torsion angles. Alternatively as shown in Fig. 1.9, dihedral angle restraints can be obtained exploiting the information contained in the secondary chemical shifts of the heteronuclei in the backbone (N, $\mathrm{C} \alpha$ and $\mathrm{CO})$, which in turn depend on $\varphi$ and $\psi$. The secondary chemical shift is defined as the difference between the measured chemical shift and the chemical shifts in a random-coil (e.g., unstructured) protein and represents that component of the chemical shift that is induced by the three-dimensional structure. Dihedral angle restraints can be derived automatically with the TALOS [Cornilescu, Delaglio 1999] program, which relates 
the backbone secondary shifts with an internal database of high-resolution protein structures. TALOS strategy was used in this work to derive dihedral angle restraints.

\subsubsection{Residual Dipolar Couplings}

Recently, a different source of structural information has been used for structure determination: the magnetic dipole-dipole coupling between spin $1 / 2$ nuclei (e.g. ${ }^{1} \mathrm{H}$,

${ }^{13} \mathrm{C},{ }^{15} \mathrm{~N}$ ). These dipolar couplings contain information on the orientation of internuclear vector relative to the magnetic field and distance between the involved nuclei. They have proven invaluable for improving the accuracy of macromolecular NMR structure determination, for independently validating their accuracy, for refining crude homology of proteins and for defining intermolecular interactions. In addition, dipolar couplings can be used to search for homologous structures or substructures in a structure database and potentially could replace the time consuming regular NOE data collection and analysis process [Permi and Annila 2001; Meiler, Peti and Griesinger 200; Gaemers and Bax 2001].

The use of residual dipolar couplings (RDCs) to enhance the information available from high-resolution NMR spectra has a long history. Its roots can be traced to the substantial amount of NMR done in magnetic-field-aligned liquid crystals in the early 1960's by Saupe and Englert [Saupe and Englert 1963]. They demonstrated that an organic molecule dissolved in a nematic liquid crystalline phase exhibits quite strong alignment when placed in an NMR magnet. Thoughts about application to biomolecules in solution arose more than 15 years ago with the observation that isolated molecules with sufficient anisotropic susceptibilities would adopt slightly non-isotropic orientational distributions when placed in a high magnetic field. The major breakthrough with respect to any potentially routine use of dipolar couplings 
for biomolecular structure determination was the demonstration that adjustable degrees of alignment could be achieved by placing the molecule under investigation into a dilute, aqueous liquid crystalline phase of dihexanoyl phosphatidylcholine (DHPC) and dimyristoyl phosphatidycholine (DMPC) [Sanders and Prestegard 1993; Tjandra and Bax 1997].

The application described above relies on the fact that anisotropic contributions to nuclear interactions, e.g. dipolar interaction between pairs of magnetically active spin $1 / 2$ nuclei, do not average to zero when the molecules of have a preferred orientation. The net alignment of the molecules of interest, which can be introduced by a liquid crystalline medium, is on the order of $10^{-3}$ and is fundamental to the success of RDC based studies.

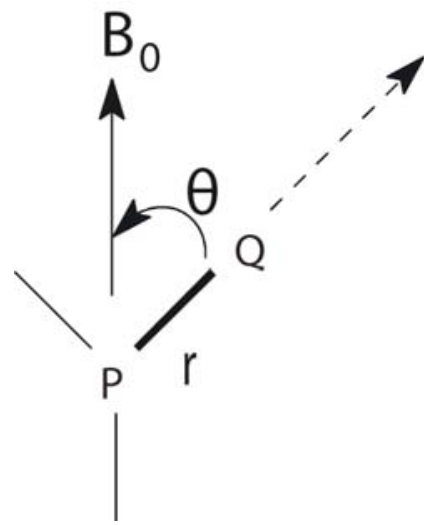

Figure 1.10: Dipolar coupled spin pair. The bond length $r$ is assumed to be fixed and the primary variable is the angle $\theta$ between magnetic field $\mathrm{B}_{0}$ and the internuclear vector.

Dipolar couplings are potentially quite large interactions, caused by the magnetic field produced by one nucleus (e.g. nucleus Q) affecting the energy of another nucleus (e.g. nucleus P) (Figure 1.10). The components orthogonal to the magnetic field $\mathrm{B}_{0}$ have a negligible effect on the total magnitude of the vector sum of the external and the dipolar field. Thus only the $\mathrm{z}$ component of the dipolar field of nucleus $\mathrm{Q}$ will change the resonance frequency of nucleus $\mathrm{P}$ by an amount that depends on the internuclear distance and on the orientation of the internuclear vector relative to $\mathrm{B}_{0}$. For a fixed 
orientation of the vector, nuclear spin $\mathrm{P}$ can decrease or increase the total magnetic field at nucleus $\mathrm{Q}$, depending on whether $\mathrm{P}$ is parallel or antiparallel to $\mathrm{B}_{0}$. In an ensemble of molecules half of the $\mathrm{P}$ nuclei will be parallel to $\mathrm{B}_{0}$ and the other half antiparallel, and Q will show two resonances separated in frequency by,

$$
D^{P Q}=D_{\max }^{P Q}\left\langle\frac{3 \cos ^{2} \theta-1}{2}\right\rangle
$$

where $\theta$ is the angle between the internuclear vector and B0, the brackets $<>$ denote time or ensemble averaging, and

$$
D_{\max }^{P Q}=-\frac{\mu_{0}}{4 \pi} \gamma_{P} \gamma_{Q} \frac{\hbar}{2 \pi}\left\langle\frac{1}{r_{P Q}^{3}}\right\rangle
$$

is the doublet splitting that applies for the case where $\theta=0$ and where $\mu_{0}$ is the magnetic permittivity is vacuum; $\hbar=\mathrm{h} /(2 \pi)$, in which $\mathrm{h}=$ Planck's constant; $\gamma_{\mathrm{p}}$, gyromagnetic ratio of nucleus $\mathrm{P} ; \gamma_{\mathrm{Q}}$, gyromagnetic ratio of nucleus $\mathrm{Q} ; \mathrm{r}_{\mathrm{PQ}}$, the internuclear distance between nucleus $\mathrm{P}$ and Q. Equation (1.12) shows the dipolar splitting, $\mathrm{D}^{\mathrm{PQ}}$, provides direct information on the angle $\theta$.

Knowing $\theta$ for a bonded pair of nuclei, e.g. ${ }^{1} \mathrm{H}^{-}{ }^{15} \mathrm{~N}$, can be very useful in defining a molecular structure. The brackets around the $\theta$ dependent term, however, denote a time average. Normally in solution we assume isotropic sampling (that the time average results a molecular tumbling that uniformly samples directions in space). Consequently, for any pair of nuclei, the $3 \cos ^{2} \theta-1$ term averages to zero and no residual dipolar coupling can be measured [Prestegard 1998]. For this reason NMR spectroscopists were relegated to measure dipole-dipole interaction indirectly through spin relaxation based phenomena such as NOE until 1997, when Tjandra and Bax demonstrated the use of a liquid crystalline medium to introduce a tunable degree of alignment. 
One important property of residual dipolar couplings between two nuclei $\mathrm{P}$ and $\mathrm{Q}$ that have scalar coupling is that dipolar coupling $\left(\mathrm{D}^{\mathrm{PQ}}\right)$ is added to the isotropic coupling constant $\left(\mathrm{J}^{\mathrm{PQ}}\right)$. Therefore, when measuring the effective dipolar coupling under anisotropic conditions $\left(\mathrm{E}^{\mathrm{PQ}}\right)$, the spin-spin couplings needs to be subtracted from the measured coupling to obtain the desired dipolar coupling.

$$
\mathrm{E}^{\mathrm{PQ}}=\mathrm{J}^{\mathrm{PQ}}+\mathrm{D}^{\mathrm{PQ}}
$$

Often the one-bond dipolar couplings (e.g. $\mathrm{NH}, \mathrm{C}_{\alpha} \mathrm{H}_{\alpha}, \mathrm{C}_{\alpha} \mathrm{C}^{\prime}, \mathrm{C}^{\prime} \mathrm{N}$ ) are measured, but also two-bond and three-bond dipolar couplings can be measured. One-bond dipolar couplings are easier to interpret because the inter-atomic distance is known and the magnitude of the dipolar interaction is relatively large [De Alba and Tjandra 2002].

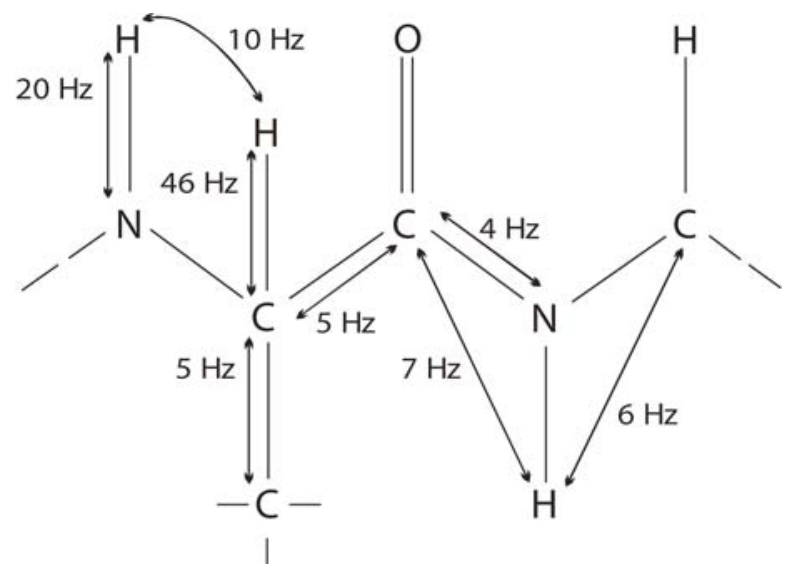

Figure 1.11: The predicted maximum absolute residual dipolar contributions for different nuclei in the protein main-chain at $0.1 \%$ levels of alignment.

The NMR methods used for measuring residual dipolar couplings can be divided into two general categories: frequency resolved methods (J-resolved) and intensity-based experiments (J-modulated). In frequency resolved methods the separation of the peaks is measured in a frequency domain. In intensity based experiments the coupling is extracted from the resonance intensity rather than from the experimental splitting. The Principle underlying the J-modulated experiments is to pass the observed signal 
through a period in which the intensity is modulated by a known function of the spinspin coupling [Brunner 2001;Prestegard, Al-Hashimi 2000]. An intensity-based experiment is particularly useful in case of overlap problems in the described coupled spectra because of the increased number of signals due to the doublet splitting. In addition, J-modulated spectroscopy is a good alternative when the coupling of interest is small compared with the line width, which is often the case with ${ }^{3} \mathrm{~J}$ couplings. ${ }^{15} \mathrm{~N}$ ${ }^{1} \mathrm{H}$ splitting was measured under isotropic and anisotropic conditions using 2D IPAP${ }^{15} \mathrm{~N}-{ }^{1} \mathrm{H}$ HSQC experiments [Ottiger, Delaglio 1998]. ${ }^{1} \mathrm{H}-{ }^{15} \mathrm{~N}$ RDCs were extracted by subtraction of the ${ }^{1} \mathrm{~J}_{\mathrm{NH}}$ scalar coupling measured for the isotropic sample [Tjandra and Bax 1997]. Alternatively, ${ }^{1} \mathrm{H}_{-}{ }^{15} \mathrm{~N}$ RDCs were obtained from the ${ }^{15} \mathrm{~N}-{ }^{1} \mathrm{H}$ splittings of a modified 3D TROSY-HNCO experiment [Chou, Delaglio 2000]. The quantitative J correlation method was used to determine one-bond dipolar ${ }^{13} \mathrm{C}^{\prime}-{ }^{15} \mathrm{~N}$ couplings. Usually, these couplings are measured together with the ${ }^{1} \mathrm{H}^{-}{ }^{15} \mathrm{~N}$ RDCs [Vijayan and Zweckstetter 2005]. ${ }^{13} \mathrm{C}^{\prime}-{ }^{13} \mathrm{C}_{\alpha}$-RDCs are measured from the splitting in the ${ }^{13} \mathrm{C}^{\prime}$ dimension of a ${ }^{13} \mathrm{C}_{\alpha}$ coupled 3D HNCO experiment.

\subsubsection{Structure calculation via Simulated Annealing}

Following the sequential assignment and assignment of NOESY spectra, NOEderived distance restraints, dihedral angle restraints, residual dipolar couplings (additional restraints like hydrogen bonds, disulphide bridges, if present) are used to calculate an ensemble of structures. However, the experimentally determined distances and torsion angles by themselves are not sufficient to fully characterize a protein structure, as they are based on a limited number of proton-proton distances. Only the knowledge of empirical input data, such as bond lengths of all covalently attached atoms and bond angles, enables a reasonably exact structure determination. 
The ultimate goal of structure calculation algorithms is to operate an optimization of the simultaneous agreement of an atomic model with the observed data and with $a$ priori chemical information. Most algorithms used for structure calculation aim to find the global minimum of a hybrid energy function $\mathrm{E}$ (target function), which includes a priori chemical knowledge of the system (the force field, defining bond lengths, bond angles, improper angles and non-bonded interactions) and experimental data (the structural restraints):

$$
\begin{aligned}
& E=E_{\text {chem }}+E_{\text {exp }}=\sum_{i} w_{i} E_{i}= \\
& w_{\text {cov alent }} E_{\text {cov alent }}+w_{\text {angle }} E_{\text {angle }}+w_{\text {float }} E_{\text {float }}+w_{v d W} E_{v d W}+ \\
& w_{N O E} E_{N O E}+W_{\text {dihed }} E_{\text {dihed }}+w_{R D C} E_{R D C}+\ldots
\end{aligned}
$$

$E_{\text {chem }}$ in Equation 1.15 contains energy terms for covalent bonds, bond angles, chirality, planarity and nonbonded repulsion are all approximated by a harmonic function. In contrast, non-covalent van-der-Waals attraction forces and electrostatic interactions are simulated by an inharmonic Lennard-Jones potential or Coulomb potential, respectively. Nonbonded repulsions are described by a quartic potential [Stein, Rice 1997]. $E_{\text {exp }}$ contains energy terms describing the experimental structural restraints.

The target function $\mathrm{E}$ is a function of many molecular parameters, most importantly of atomic coordinates. The large number of variables makes this function very complex and originates the so-called 'multiple minima problem': the target function contains many local minima in addition to the global minimum. The standard minimization methods [Brunger and Nilges 1993] tend to steer the system into local minima and frequently fail to reach the global minimum if the starting model is far away from the correct one. The sampling of a larger conformational space can be achieved by using Simulated Annealing (SA) optimization techniques [Brunger, Adams 1997;Brunger and Nilges 1993;Kirkpatrick, Gelatt 1983], which has the 
important property of enabling the system to overcome local minima (Figure 1.12). Monte Carlo-based and molecular dynamics-based SA have been used in structure prediction, molecular modeling, X-ray refinement and NMR structure determination for many years and have had a large impact in structural biology.

Structure calculation based on SA molecular dynamics consists in the integration of Newton's equation of motion:

$$
\frac{d^{2} \vec{r}_{i}}{d t^{2}}=-\frac{c}{m_{i}} \frac{\nabla E}{\vec{r}_{i}}
$$

where $\vec{r}_{i}$ is the vector of Cartesian coordinates, $\mathrm{m}_{\mathrm{i}}$ the mass of the atom $\mathrm{i}, \mathrm{c}$ is a constant and $\nabla E$ is the gradient of the target function. The global minimum of the target function $\mathrm{E}$ is searched by reducing the temperature of the system after a hightemperature phase during a molecular dynamics simulation. In this context, the parameter 'temperature' has no physical meaning, but is simply a measure of the probability of the macromolecule to cross an energy barrier (i.e. its kinetic energy). Temperature control is performed by coupling the system with a thermal bath [Berendsen, Postma 1984]. As a result, temperature coupling will cause "heat" (kinetic energy) to be added or removed from the system, as it is needed to maintain the temperature.

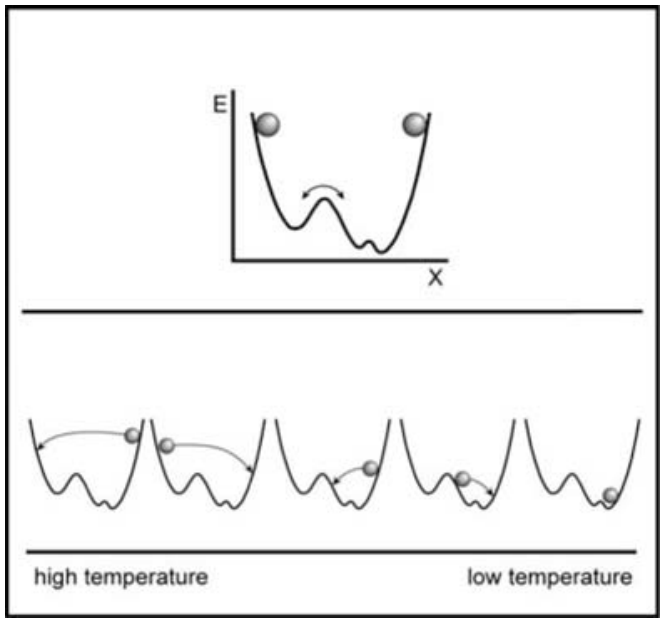

Figure 1.12: Compared to standard minimisation methods, SA allows the system to overcome local energy barriers, reducing the risk of the molecule to get trapped in local minima of the target function. 
Energy terms for distance- and angle restraints are usually provided by specifying a lower and upper limit, L and U. During simulated annealing, a violation is generated whenever the distance between the two atoms is not contained between the boundaries, leading to an increase in energy of the system. Typically, when restraints from manually assigned peaks are provided as input, energy terms for NOE-based distance restraints and dihedral angle restraints are present in the form of flatbottomed parabolic functions (Figure 1.13). The flat bottom is delimited by L and U:

$$
\begin{gathered}
\mathrm{E}_{\text {dis_res }}= \begin{cases}(\mathrm{L}-\mathrm{d})^{2} & \text { if } \mathrm{d}<\mathrm{L} \\
0 & \text { if } \mathrm{L}<\mathrm{d}<\mathrm{U} \\
(\mathrm{d}-\mathrm{U})^{2} & \text { if } \mathrm{U}<\mathrm{d}\end{cases} \\
\mathrm{E}_{\text {dih_res }}= \begin{cases}(\mathrm{L}-\phi)^{2} & \text { if } \phi<\mathrm{L} \\
0 & \text { if } \mathrm{L}<\phi<\mathrm{U} \\
(\phi-\mathrm{U})^{2} & \text { if } \mathrm{U}<\phi\end{cases}
\end{gathered}
$$

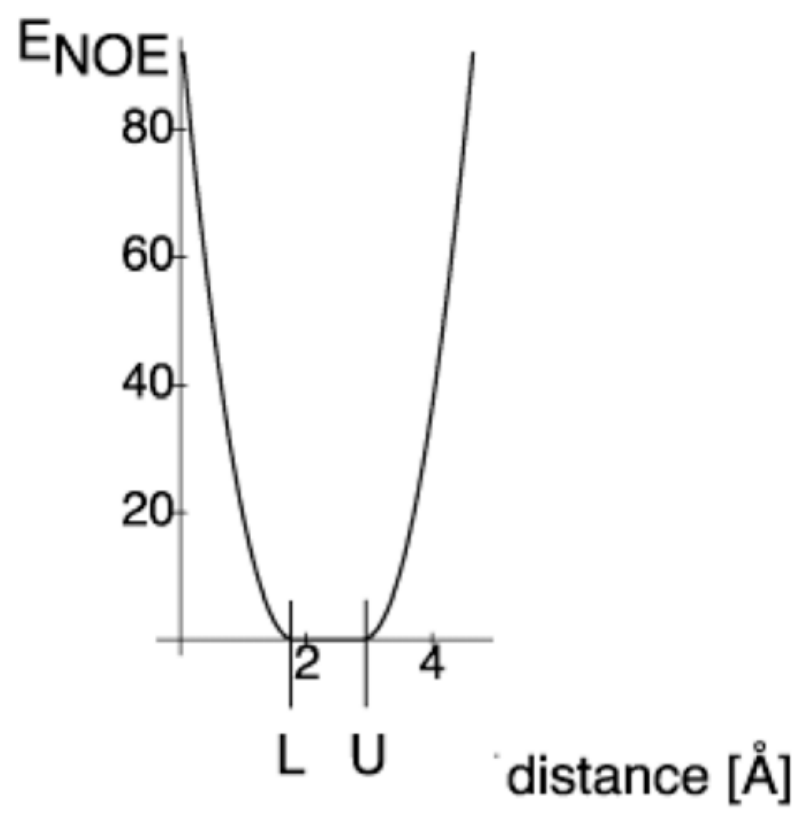

Figure 1.13: Standard "flat-bottom" potential used for NOE-derived distance restraints.

Structure calculations using iterative methods for automated cross-peak assignment [Linge, Habeck 2003;Linge, O'donoghue 2001] are characterized by large violations 
of experimental distance restraints in the atomic model during the early iterations. Hence, a linear function is substituted in Equation 1.14 for large violations (Equation 1.15), in order to avoid numeric instabilities arising from the high penalty for large violations (Figure 1.14).

$$
E_{\text {dis_res }}= \begin{cases}(L-d)^{2} & \text { if } d<L \\ 0 & \text { if } L<d<U \\ (d-U)^{2} & \text { if } U<d<S \\ A(d-U)^{-1}+B(d-U)+C & \text { if } d>S\end{cases}
$$

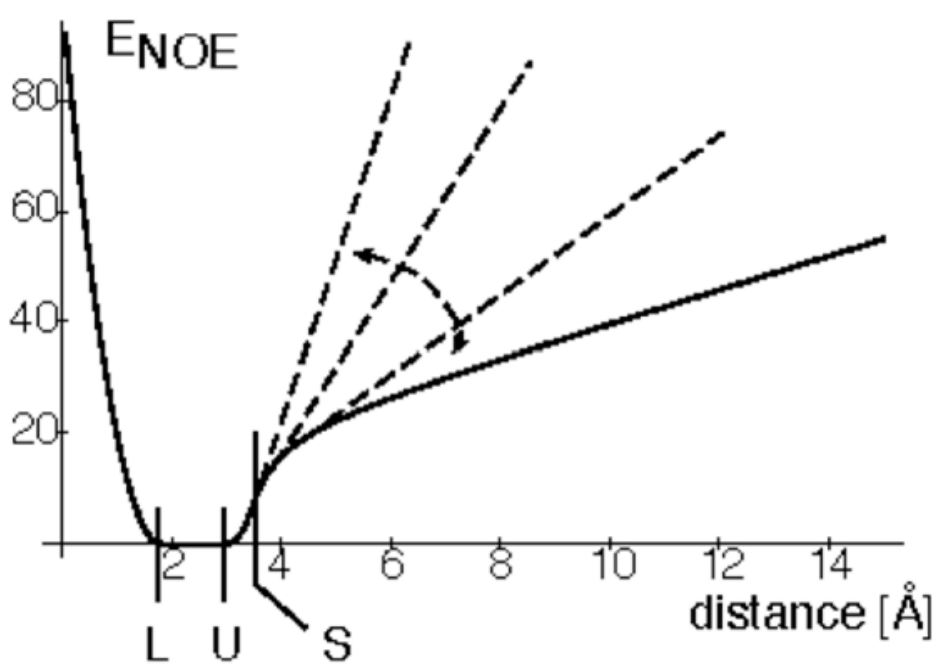

Figure 1.14: Soft potential for NOE-derived restraints used in iterative methods for automated cross-peak assignment.

Similar minimization procedure is used for RDCs and other experimental restraints during the structure calculation. Without the experimentally determined distance, torsion angles constraints and RDCs from the NMR spectra, the protein molecule can adopt a huge number of conformations due to the free rotation around its chemical bonds (except for the peptide bond, the $\mathrm{N}-\mathrm{C}^{\alpha}$ bond and $\mathrm{C}^{\alpha}-\mathrm{C}^{\prime}$ bond). All these possible conformations are summed up in the so-called conformational space. Therefore, it is important to identify as many constraints as possible from the NMR 
spectra to restrict the conformational space as much as possible, thus getting close to the true structure of the protein. In fact, the number of constraints employed is more important than the accuracy of proton-proton distance. The precision with which a structure can be calculated is directly related to the number of experimental restraint used to generate it. Structure of low resolution may be obtained with as few as five restraints per residue, whereas the most precise structures obtained from NOE constraints alone may have up to 15 restraints per residue.

The result of simulation is a minimum energy protein structure, but it cannot be excluded that this structure is stuck in a local minimum without ever reaching global minimum, which is marginally lower in energy. Therefore, about twenty different starting structures with random folds are used, which reach their final structure via different paths in energyhyperspace. These resulting structures are iteratively re-used as starting structures for another SA with slightly changed input protocols, until no further reduction in global energy is observed and the structures converge in conformational space.

After the structural calculations a family of structures is obtained instead of an exactly defined structure. This family spans out a relatively narrow conformational space. Therefore, the quality of a NMR structure can be defined by the mean deviation of each structure from this family (RMSD) from an energy minimized mean structure, which has to be calculated previously. The smaller the deviations from this mean structure, the narrower the conformational space. Another definition of RMSD is to compare pair wise the structures of a family and calculate the mean of these deviations.

The RMSD is different for different parts of the protein structure: Regions with flexible structure or without secondary structure (loops) show a larger deviation than those with rigid and well defined secondary structure. This higher RMSD in 
loops results in first instance from the smaller number of distance constraints for these parts of the protein structure. Additionally it can originate from real flexibility, but this diagnosis can only be confirmed by measuring the relaxation times for the protein.

\subsection{Solid-State NMR}

Solid-state NMR has long been the poor cousin of liquid state NMR in structural biology so it is important to know why one might be interested in using such a technically difficult method. Considerable advances have meant that solid state NMR is now readily applicable to biological systems and a wide variety of information can be elucidated with solid-state NMR. How far the technique can be pushed, its limitations and how they might be removed is an area of intense discussion and has seen rapid progress in recent years.

Solution-state NMR has constantly developed methods since the first proteinstructure determination in 1985 by K. Wüthrich and currently a large toolbox of multidimensional NMR experiments, labeling schemes, automated or semi-automated programs exist for sequential assignment and structure calculation. Proteins up to $30 \mathrm{kDa}$ can be routinely studied by solution-state NMR and successful applications have been demonstrated for proteins with molecular weights up to $100 \mathrm{kDa}$. This limitation in solution-NMR appears due to the increase of the correlation time with the molecular weight ('correlation time problem') that shortens the transverse relaxation time and degrades spectral resolution. The problem becomes even more apparent in the case of membrane proteins where the size of the lipid-protein assemblies (micelles, bicelles, liposomes) can easily reach or exceed the above limits even for small proteins.

The situation is different in solid-state NMR. As implied by the name, solid- 
state NMR is used for solids, nearly solids, or strongly anisotropic or immobilized proteins, that is, samples where the reorientation of a molecule is very slow or nonexistent, as is the case for microcrystalline powders of soluble proteins or membrane proteins reconstituted in lipid bilayers. Therefore, solid-state NMR is a technique to investigate proteins whenever the dissolution of the sample is not possible or desirable and the X-ray approach is also not feasible. This may involve: 1) insoluble proteins, 2) proteins which aggregate in solution, 3) membrane-bound proteins, often insoluble or structurally altered in aqueous solution, in their synthetic or natural membrane environment. Compared to solution NMR, solid-state NMR has the disadvantage of a much lower resolution in terms of achievable line-widths relative to the chemical shift range. Conversely, molecular tumbling is not a band-narrowing mechanism in solidstate NMR; therefore, the size of the protein does not influence the line-width and, thus, is not an intrinsic limitation on resolution. In addition, fast internal dynamics may improve resolution.

The chemical shift tensor and the dipolar interaction tensor all contain an orientation dependent factor:

$$
3 \cos ^{2} \theta-1
$$

Most of the experimental techniques used in solid-state NMR have been developed to achieve (1) line-narrowing or (2) signal enhancement. Intrinsic broad lines due to the anisotropy of NMR interactions and short T2 relaxation times characterize solid-state NMR. Anisotropically broadened lineshapes affect mainly randomly oriented (sometimes called 'powder') samples that are also the most interesting for biological applications. There are line-narrowing methods that averageout anisotropic interaction either in real space by magic angle spinning (MAS) [Mehring 1979; Maricq 1979] or in spin-space [Lee and Goldburg 1965]. Motional averaging, an intrinsic property of liquids, can be simulated by rapid sample spinning 
at the magic angle at $\theta_{\mathrm{m}}=54.733^{\circ}$ (Figure 1.15) relative to the magnetic field $\mathrm{B}_{0}$, which leads to the vanishing of the anisotropy factor contained in Equation 1.19.

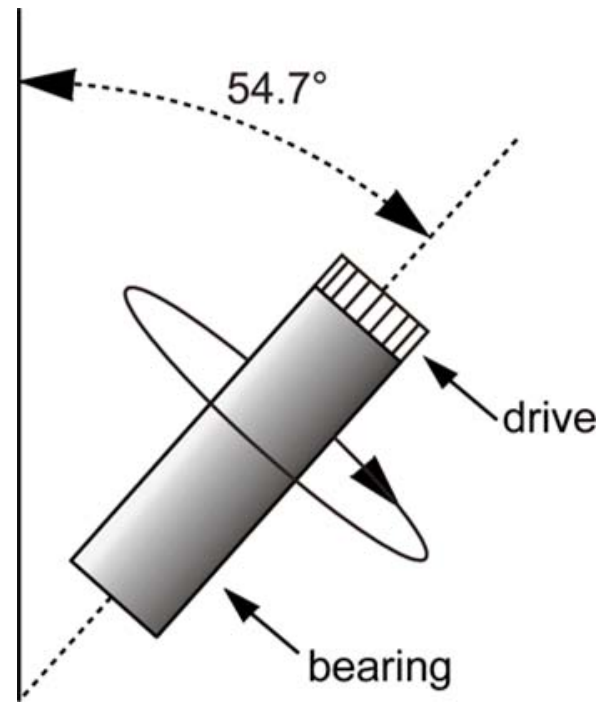

Figure 1.15: Principle of the magic-angle spinning (MAS) technique. The rotor containing the sample is tilted at $54.7^{\circ}$ with respect to the external magnetic field

Anisotropic interactions, like dipolar couplings and the anisotropic part of the chemical shift can be averaged out if the spinning is sufficiently rapid. Thus, also for solid-state samples it is possible to obtain, with some limitations, an isotropic Hamiltonian, giving rise to relatively narrow signals with absorption line shapes. In practice, to completely average out the anisotropies, the spinning rate has to be significantly larger than the interaction strength (in $\mathrm{Hz}$ ). This can be easily achieved with interactions such as homonuclear and heteronuclear dipolar couplings not involving protons $(<10 \mathrm{kHz})$.

In biological solid-state NMR, homonuclear and heteronuclear correlations between ${ }^{13} \mathrm{C}$ and ${ }^{15} \mathrm{~N}$ are most commonly measured. Such correlations are mostly mediated by dipolar interactions. The current strategy for uniformly labeled proteins in MAS solid-state NMR relies heavily in ${ }^{13} \mathrm{C}$ (detected) and ${ }^{15} \mathrm{~N}$ nuclei, making isotope labeling mandatory [Kainosho 1997]. While resonance assignment methods in the solution-state make extensive use of the J-coupling to direct polarization along the 
polypeptide chain [Ikura, Kay and Bax 1990;Ikura, Kay, Tschudin 1990], solid-state NMR mixing schemes can employ in principle both through-bond or through-space transfer mechanism to achieve sequential resonance assignments under MAS conditions. The residue type are identified in $\left({ }^{13} \mathrm{C},{ }^{13} \mathrm{C}\right)$ homonuclear correlation spectra based on unique spin connectivities and distinct ${ }^{13} \mathrm{C}$ chemical shifts of each residue, while sequential assignment is obtained from combination of heteronuclear NCACX and NCOCX (where X denotes second spin involved in magnetization transfer step) spectra that link neighbor residues via the common amide ${ }^{15} \mathrm{~N}$ nucleus [Baldus 2002; Tycko 1996]. Sequential assignments can be probed also in ${ }^{13} \mathrm{C}-{ }^{13} \mathrm{C}$ correlation spectra under specific, so called 'weak coupling conditions' [Seidel, Lange 2004], or in ${ }^{15} \mathrm{~N}-{ }^{15} \mathrm{~N}$ correlations when possible [Van Rossum, Castellani 2003]. To improve resolution and obtain long range constraints in spin diffusion [Bloembergen 1949;Suter and Ernst 1985] spectra, special ${ }^{13} \mathrm{C}$ labeling schemes have been proposed [Castellani, Van Rossum 2002]. Finally, sequential resonance assignments can also be obtained from proton-proton [Lange, Luca 2002] or ${ }^{1} \mathrm{H}-{ }^{13} \mathrm{C}$ mediated [Etzkorn, Bockmann 2004] correlation spectroscopy.

\subsubsection{Protein structure determination via solid-state NMR}

Recently, a simple methodology was developed to investigate protein structures from solid-state MAS NMR data ( $\S$ Chapter 3 ). This approach bears many parallels with the strategy used for structure determination of proteins via solution NMR (§ 1.2.1).

\subsubsection{Collection of structural restraints}

For structure determination of proteins by MAS NMR it is first necessary to perform a

resonance assignment. Subsequently, distance measurements are done, for which 
there are different approaches in solid-state NMR. In liquid state NMR, the collection of distance restraints for structure calculation protocol relies on the NOE between pairs of protons. In solid-state NMR, for uniformly ${ }^{13} \mathrm{C}$ labeled samples, homonuclear ${ }^{13} \mathrm{C}-{ }^{13} \mathrm{C}$ and heteronuclear ${ }^{13} \mathrm{C}-{ }^{15} \mathrm{~N}$ correlations are more commonly used for extracting distance information, from proton-driven-spin-diffusion (PDSD) [Castellani, Van Rossum 2002] types of spectra, as the large size of dipolar protonproton interactions makes it challenging to measure ${ }^{1} \mathrm{H}-{ }^{1} \mathrm{H}$ correlations. The crosspeak volumes in the PDSD spectra show either an $\mathrm{r}^{-3}$ or $\mathrm{r}^{-6}$ dependency. By assigning

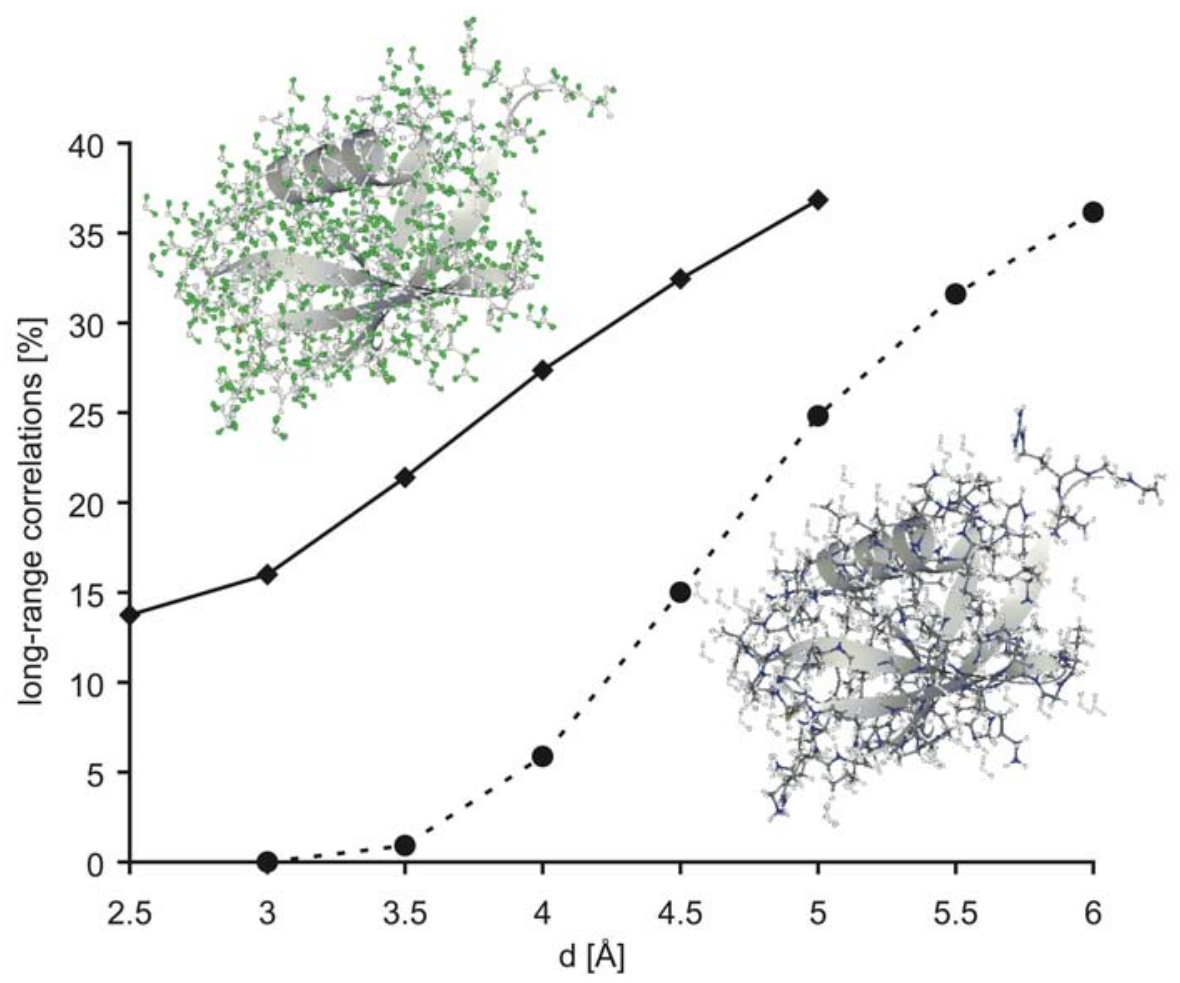

Figure 1.16: Statistical analysis of all proton-proton and carbon-carbon distance constraints up to distance $d$ in ubiquitin. The fraction of long-range distances (residue difference $>4$ ) is shown.

cross-peaks, a large number of inaccurate ${ }^{13} \mathrm{C}-{ }^{13} \mathrm{C}$ distance restraints in the range of 2$7.5 \AA$ are derived. As an alternative to the measurement of carbon or nitrogen distances, a set of multidimensional NMR experiments for structure elucidation of proteins under MAS conditions has been proposed, which relies on the measurement of proton-proton distances [Heise, Seidel 2005] (used in this thesis). It uses proton 
homonuclear transfers, bracketed by ${ }^{13} \mathrm{C}$ and/or ${ }^{15} \mathrm{~N}$ evolution times for the sake of spectral resolution. This scheme is able to produce high-resolution spectra connecting carbon or nitrogen spins through space [Lange, Luca 2002;Lange, Seidel 2003]. Compared to ${ }^{13} \mathrm{C}-{ }^{13} \mathrm{C}$ or ${ }^{13} \mathrm{C}-{ }^{15} \mathrm{~N}$ correlations in proteins, proton-proton interactions are more abundant and, because of their peripheral nature, contain a larger fraction of long-range contacts for the shortest internuclear distances [Baldus 2007].

Finally, from the conformational dependent backbone chemical shifts $\left({ }^{15} \mathrm{~N},{ }^{13} \mathrm{C}_{\alpha}\right.$ and ${ }^{13} \mathrm{C}_{\beta}$ ), a set of dihedral angle restraints can be obtained with the TALOS program (§1.2.4.2).

\subsubsection{Structure calculation via Simulated Annealing}

Structure calculation approach is similar as described in $(\S 1.2 .5)$. 


\section{Chapter 2}

\section{Fast High-resolution Protein Structure Determination by Using Unassigned NMR Data}

\subsection{Introduction}

Structural studies of proteins are critical for understanding biological processes at the molecular level. NMR spectroscopy provides high-resolution structural information of biomolecules in near-physiological conditions. Although significant improvements were achieved in NMR spectroscopy in the last 20 years [Wuthrich 2003], the increase in genome sequencing data has created a need for rapid and efficient methods of NMR-based structure determination [Abbott 2005;Montelione, Zheng 2000]. In contrast to X-ray crystallography, growing of suitable crystals is not required for NMR spectroscopy, and the time-consuming steps are data acquisition and data analysis. NMR data acquisition can be accelerated significantly, when highly sensitive spectrometers equipped with cryogenic probes are combined with newly developed methods for sampling chemical shifts in multidimensional NMR experiments. For approximately $1 \mathrm{mM}$ protein solutions, the NMR data, which are required for threedimensional (3D) structure determination, may be obtained in one to nine days, depending on the size and properties of the protein [Liu, Shen 2005]. Therefore, data analysis and in particular the requirement to assign chemical shifts and inter-residue correlations to specific atoms is the major bottleneck of rapid NMR-based structure determination.

Good progress has been made towards prediction of high-resolution 3D protein structures from amino acid sequences. Using improved conformational sampling methods, structural models were predicted that had a $\mathrm{C}_{\alpha}$-root mean square 
deviation (rmsd) to the native structures of less than $1.5 \AA$ [Bradley, Misura 2005]. However for many proteins the quality of predictions was insufficient and prediction of the detailed structures of functionally relevant parts remains a formidable challenge.

These limitations of $a b$ initio structure prediction may be overcome, when a limited number of easily accessible NMR data is combined with ab initio methods. For example, medium-resolution folds can be determined rapidly from chemical shifts and residual dipolar couplings (RDCs) [Delaglio, Kontaxis 2000], especially when combined with the de novo structure prediction algorithm Rosetta [Jung, Sharma 2004;Meiler and Baker 2003; Rohl and Baker 2002]. To obtain high-resolution protein structures, experimental distance information is required. The distance information can be extracted from Nuclear Overhauser effect (NOE) spectra and can be obtained from NOESY (NOE spectroscopy) spectra.

NOESY spectra provide large number of distances between protons that are close in space (not farther than $5 \AA$, approximately). Unfortunately, the interpretation of NOESY spectra is never straightforward. Signal degeneracy leads to several assignment options for many peaks. With larger proteins, these effects are usually so dramatic, that a complete manual interpretation of the spectra is hampered. Consequently, the automation of this step, towards high-resolution structure, has become an issue of great interest in the last years. The distance restraints can be extracted from NOESY spectra with little manual intervention, when assignment of NOE peaks and structure calculation are performed iteratively. Today, several software packages exist for a more or less fully automated NOE assignment. ARIA [Linge, Habeck 2003;Linge, O'donoghue 2001], CANDID [Herrmann, Guntert 2002], DYANA [Guntert, Mumenthaler 1997], KNOWNOE [Gronwald, Moussa 2002], NOAH [Mumenthaler and Braun 1995;Mumenthaler, Guntert 1997], 
AUTOSTRUCTURE [Moseley, Monleon 2001;Moseley and Montelione 1999] and have recently been reviewed [Guntert 2003].

All these approaches require the sequence-specific resonance assignment and lists with cross-peaks from NOESY spectra as input. These automated procedures follow the same general scheme of iteratively assigning the multi-dimensional NOESY data and structure calculation. A key-step towards complete automation was the introduction of Ambiguous Distance Restraints (ADR) [Nilges 1993;Nilges 1995] into structure calculation strategies based on simulated annealing. ARIA uses such an ambiguous constraint strategy, involving multiple ambiguous distance constraints for each ambiguous NOESY peak. The program CANDID, combined with DYANA, also uses ambiguous constraint strategies but, in addition, employs network anchoring and constraint-combination methods, minimizing deleterious effects when this correctness assumption is not satisfied. Initial structures are first built using ambiguous constraint strategies and then iteratively refined. Therefore, it is important to obtain a wellconverged initial fold for the rest of the cycles to achieve correct structures. To converge to the correct structure, however, a nearly complete and error-free manual assignment of backbone and side chain chemical shifts is essential [Jee and Guntert 2003].

Alternatively, it may be possible to obtain a $3 \mathrm{D}$ protein structure in the absence of any chemical shift assignments from the distance information provided in NOESY spectra. Several attempts have been made to devise a strategy for NMR protein structure determination that circumvents the tedious chemical shifts assignment step. The underlying idea of assignment-free NMR structure calculation methods is to exploit the fact that NOESY spectra provide distance information even in the absence of any chemical shift assignments. This proton-proton distance information can be exploited to calculate a spatial proton distribution. Since there is 
no association with the covalent structure at this point, the protons of the protein are treated as a gas of unconnected particles. Provided that the emerging proton distribution is sufficiently clear, a model can then built into the proton density in a manner analogous to X-ray crystallography in which structural model is constructed into the electron density. The most recent approach to NMR structure determination without chemical shift assignment is the CLOUDS [Grishaev and Llinas 2002a;Grishaev and Llinas 2002b] approach. For the first time, the feasibility of the method has been demonstrated using experimental data rather than simulated data sets. The CLOUDS method relies on precise and abundant inter-proton distance constraints calculated via a relaxation matrix analysis of set of experimental NOESY cross peaks. It showed that assignment-free NMR structure calculation could successfully generate $3 \mathrm{D}$ protein structures from experimental data. Nevertheless, in the course of a de novo structure determination it may not be straightforward to produce a NOESY peak list of the completeness and quality used for these test calculations. Unfortunately, it was assumed that the NOEs could be unambiguously identified, as it is always not feasible for the automation. Up to now, no robust method to determine high-resolution protein structure from unassigned NMR data has been reported.

Herein, we present a method termed, FastNMR (Fast Structure determination by NMR) [Korukottu, Bayrhuber 2007], which enables automatic, high-resolution NMR structure determination of domain-sized proteins from unassigned NMR data. We combined MARS (automatic backbone assignment program), RosettaNMR ( $a b$ initio structure prediction program from limited number of NMR data) and CYANA (automatic NOE assignment and structure calculation program) to facilitate the highresolution structure determination. Simultaneous backbone assignment and fold determination was followed by automatic NOE assignment and structure calculation 
using the initial fold. FastNMR differs from other approaches in that it starts from unassigned chemical shifts, NOEs and residual dipolar couplings (RDCs), avoids wrong structures by cross-validation, works for real experimental data, requires only a limited number of NMR spectra, requires no manual assignment of chemical shifts or inter-residue correlations and produces high-resolution $(<1 \AA)$ structures.

This new method was tested on two proteins and for the first time using an automatic structure determination approach, the de novo structure of the 65-residue protein cone snail neurotoxin Conkunitzin-S2 (Conk-S2) was determined automatically. 


\subsection{Materials and Methods}

A simplified flowchart of FastNMR is shown in Figure 2.1. In short, FastNMR determines the backbone resonance assignment and backbone fold of the protein by iterative assignment and structure calculation, in stage A, starting from unassigned backbone chemical shifts and residual dipolar couplings (RDCs). In stage B, experimental side chain chemical shifts are assigned by comparison with values that are back-calculated from the protein backbone fold obtained in stage A. In combination with the stage A backbone conformation, the assigned side chain chemical shifts enables an automatic NOE assignment that is performed iteratively with structure calculation. In stage $\mathrm{C}$, the NOE-based structure is refined in the presence of all the assigned restraints including RDCs.

\subsubsection{Input of unassigned NMR data}

Experiments and the structural constraints that we obtain from the observed experimental NMR parameters are listed in Table 2.1. To obtain lists of unassigned experimental data, NMR spectra can be analyzed manually or in an automated fashion. Here, all NMR spectra were processed using NMRPipe [Delaglio, Grzesiek 1995]. Referencing of spectra, peak picking and peak grouping were performed using the public domain graphics program Sparky 3 (T. D. Goddard and D. G. Kneller, SPARKY 3, University of California, San Francisco). All chemical shifts and RDCs were grouped according to the common ${ }^{1} \mathrm{H}^{\mathrm{N}},{ }^{15} \mathrm{~N}$ chemical shifts, which can be observed in the spectra used here. 


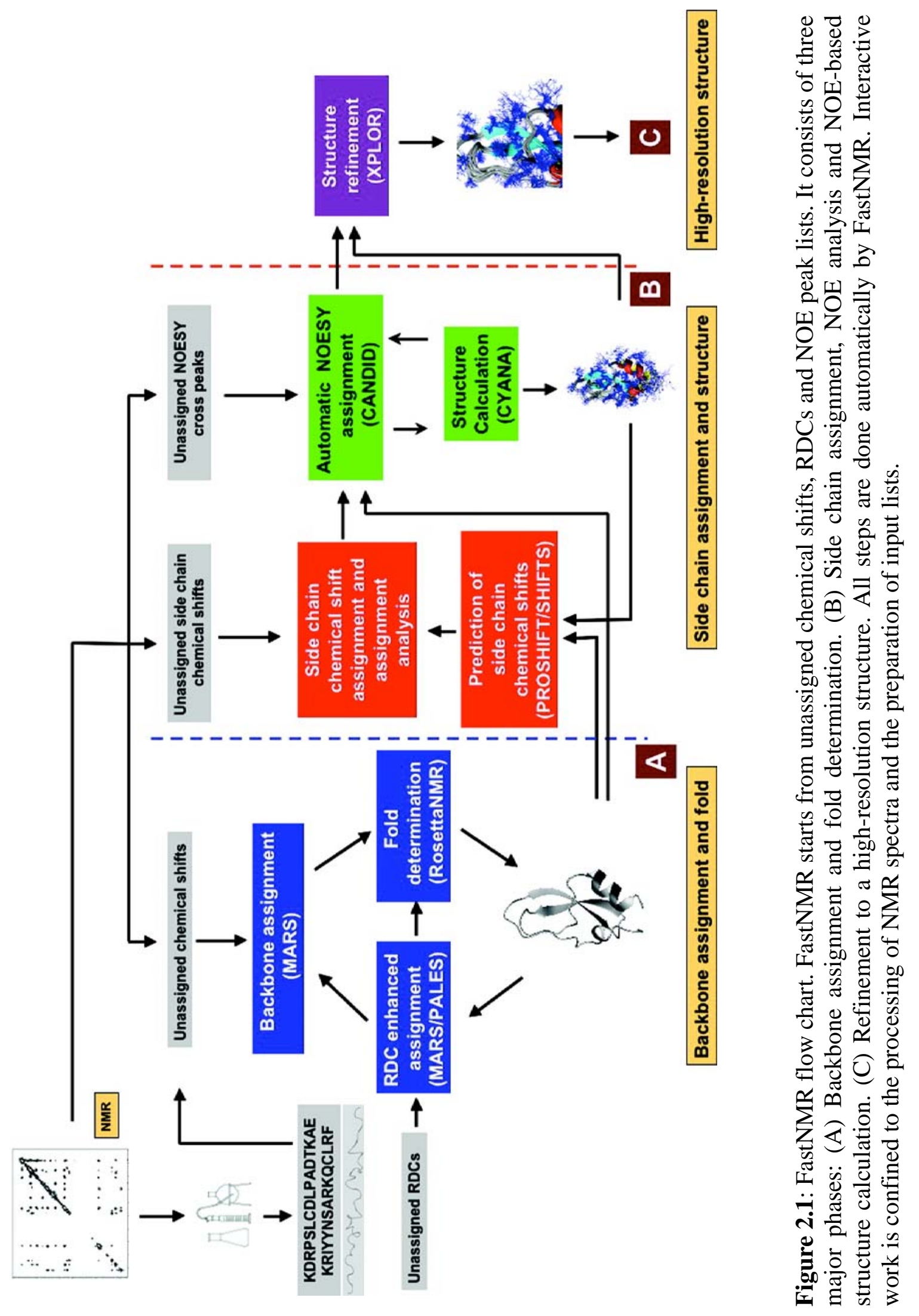




\begin{tabular}{|c|c|c|}
\hline $\begin{array}{l}\text { NMR experiment } \\
\text { (for review see [Bax and } \\
\text { Grzesiek 1993] }\end{array}$ & NMR parameters & $\begin{array}{l}\text { Structural } \\
\text { constraints }\end{array}$ \\
\hline $\begin{array}{l}\text { 3D HNCACB [Wittekind and } \\
\text { Mueller 1993] } \\
\text { 3D CBCA(CO)NH [Grzesiek } \\
\text { and Bax 1992] }\end{array}$ & $\begin{array}{c}{ }^{13} \mathrm{C}_{\alpha}{ }^{13} \mathrm{C}_{\beta},{ }^{1} \mathrm{H}_{\mathrm{N}}{ }^{15} \mathrm{~N} \\
\text { chemical shifts }\end{array}$ & $\begin{array}{l}\phi / \psi \text { Backbone dihedral } \\
\text { angles }\end{array}$ \\
\hline $\begin{array}{l}\text { 3D H(CCO)NH-TOCSY } \\
\text { [Montelione, Lyons 1992] } \\
\text { 3D C(CO)NH-TOCSY } \\
\text { [Grzesiek, Anglister 1993] }\end{array}$ & $\begin{array}{l}{ }^{13} \mathrm{C},{ }^{1} \mathrm{H} \text { chemical shifts } \\
\text { of aliphatic side chain }\end{array}$ & \\
\hline $\begin{array}{c}2 \mathrm{D}{ }^{15} \mathrm{~N}-{ }^{13} \mathrm{C}^{\gamma},{ }^{13} \mathrm{C}-{ }^{13} \mathrm{C}^{\gamma} \\
\text { difference experiments [Hu } \\
\text { and Bax 1997; Hu, Grzesiek } \\
\text { 1997] }\end{array}$ & $\begin{array}{c}{ }^{3} \mathrm{~J}_{\mathrm{CC} \gamma},{ }^{3} \mathrm{~J}_{\mathrm{NC} \gamma}, \text { scalar } \\
\text { couplings }\end{array}$ & $\chi_{1}$ angles \\
\hline $\begin{array}{l}\text { 3D RDC-TROSY-HNCO } \\
\text { [Chou, Delaglio 2000;Vijayan } \\
\text { and Zweckstetter 2005] } \\
\text { 3D RDC-CBCA(CO)NH } \\
\text { [Chou and Bax 2001;Grzesiek } \\
\text { and Bax 1992;Vijayan and } \\
\text { Zweckstetter 2005] }\end{array}$ & $\begin{array}{l}{ }^{1} \mathrm{D}_{\mathrm{N}-\mathrm{H}},{ }^{1} \mathrm{D}_{\mathrm{C}-\mathrm{N}} \\
{ }^{1} \mathrm{D}_{\mathrm{C} \alpha-\mathrm{H} \alpha},{ }^{1} \mathrm{D}_{\mathrm{C} \alpha-\mathrm{C}} \\
\quad \mathrm{RDCS}\end{array}$ & $\begin{array}{l}\text { Orientation of one- } \\
\text { bond vectors }\end{array}$ \\
\hline $\begin{array}{l}\text { 3D }{ }^{15} \mathrm{~N} \text {-edited NOESY } \\
3 \mathrm{D}{ }^{13} \mathrm{C} \text {-edited NOESY }\end{array}$ & NOE peak volumes & Distance restraints \\
\hline
\end{tabular}

Table 2.1: List of NMR experiments and structural constraints obtained from respective measured NMR parameters. 


\subsubsection{Backbone fold and assignment}

Starting from lists of unassigned chemical shifts, FastNMR obtains an initial backbone assignment of the target protein by using the MARS assignment method [Jung and Zweckstetter 2004b]. Based on the assigned residues and the corresponding chemical shifts and RDCs, molecular fragments are selected from a database of highresolution protein crystal structures. The fragments are subsequently assembled to a compact structural model using the RosettaNMR algorithm [Meiler and Baker 2003]. In the next step, the initial backbone assignment is improved by comparison of experimental RDCs with values back-calculated from the assembled structural models [Jung and Zweckstetter 2004a]. Due to the improved assignment, more chemical shifts and RDCs are available for a second round of fragment selection and assembly using RosettaNMR. The iterative process of automatic assignment and structure calculation is continued until backbone assignment and conformation have converged. 20 lowest-energy folds were used for further analysis [Jung, Sharma 2004].

The next step is to get from a protein backbone to a 3D structure including side chains. For this aim, side chains were built onto the protein backbone using the program SCWRL [Canutescu, Shelenkov 2003] to the 20 lowest-energy backbone fold obtained above.

\subsubsection{Side chain assignment and structure}

In stage $\mathrm{B}$, experimental side chain chemical shifts are assigned by comparison of experimental values with those computed from the stage A protein fold, which now has all the side chains. FastNMR uses the program SHIFTS [Osapay and Case 1994] to compute proton chemical shifts using density functional theory. Carbon chemical shifts are predicted using the program PROSHIFT [Meiler 2003], which relies on 
artificial neural networks. Predicted chemical shifts from all of the 20 lowest-energy conformers, which were transferred from stage A to stage B, were averaged. Experimental chemical shifts are then matched to the predicted values based on the minimal chemical shift difference.

Proton-proton distance constraints are derived from unassigned NOESY cross peaks detected in 3D ${ }^{13} \mathrm{C}$ - and ${ }^{15} \mathrm{~N}$-edited NOESY experiments. The structure of the protein backbone and assignment of the backbone and side-chain chemical shifts are subsequently used for NOE assignment. Using the Caliba macro in the program CYANA version 2.1 [Guntert 2003]. NOESY cross peaks are assigned automatically with the NOE assignment algorithm CANDID [Herrmann, Guntert 2002] as implemented in CYANA. In case of Conk-S1 and Conk-S2, the chemical shift tolerance window of protons and non-hydrogen atoms was set to $0.04 \mathrm{ppm}(0.02 \mathrm{ppm}$ in the direct dimension) and $0.5 \mathrm{ppm}$, respectively. The corresponding values for ubiquitin were $0.05 \mathrm{ppm}$ for protons and $0.5 \mathrm{ppm}$ for non-hydrogen atoms.

The automatically assigned distance restraints, the protein fold obtained from stage A of FastNMR, backbone dihedral angles obtained from the program TALOS [Cornilescu, Delaglio 1999] on the basis of backbone chemical shifts, and $\chi_{1}$ torsion angles are used as input for the structure calculation using the CYANA torsion angle dynamics algorithm [Herrmann, Guntert 2002]. CYANA, however, is not only used for NOE assignment. All distance constraints involving proton chemical shifts, which could not be unambiguously assigned by comparison with predicted values, are treated as ambiguous NOEs. In this way, the assignment of side chain chemical shifts is partly done as part of the automated NOE assignment. Using the NOE-based 3D structure the prediction of chemical shifts is improved and a second round of automated NOE assignment is performed. 


\subsubsection{Refinement of 3D structure to high-resolution}

Finally, in stage C of FastNMR, NOE distance restraints, which were unambiguously assigned in stage B, are evaluated. All of the NOE cross peaks assigned to protons with experimental chemical shifts are accepted. In addition, NOE distance restraints assigned to protons with predicted chemical shifts are used in the final structure refinement only if the same proton (predicted chemical shift) is assigned to two or more NOE peaks and the experimental chemical shift of the two NOE peaks differ by less than $0.1 \mathrm{ppm}$. In combination with the backbone conformation that is already established prior to the NOE analysis, this allows the determination of high-resolution protein structures. Finally, in combination with all other available experimental NMR information, the refined list of unambiguously assigned NOEs is used to refine the stage B structure in the presence of explicit water using Xplor-NIH [Schwieters, Kuszewski 2003]. The refinement follows a previously developed protocol [Nederveen, Doreleijers 2005], which was supplemented by empirical backbonebackbone hydrogen bonding potential [Grishaev and Bax 2004]. After the refinement, the 20 lowest-energy structures are selected and the automatic FastNMR structure determination is completed. 


\subsection{Results and Discussions}

FastNMR was tested on the 60-residue neurotoxin Conkunitzin-S1 (Conk-S1) and the 76-residue protein Ubiquitin. For both proteins, 3D structures as well as chemical shift assignments are known allowing best evaluation and validation for FastNMR determined structures [Bayrhuber, Graf 2006;Bayrhuber, Vijayan 2005;Cornilescu, Marquardt 1998]. In addition, the high-resolution structure of the 65-residue toxin Conkunitzin-S2, which has $72 \%$ sequence identity to Conk-S1, was determined de novo by FastNMR. Neither NMR data nor a 3D structure were previously available for Conk-S2.

Figure 2.2 shows the 20 lowest energy structures of the three proteins calculated by FastNMR and Table 2.2 show that for all three proteins FastNMR calculated high-resolution 3D structures from unassigned NMR data. The spread (root-mean square deviation within the ensemble) in the ensemble of 20 lowestenergy structures was below $0.7 \AA$ for the backbone $\left(\mathrm{N}, \mathrm{C}^{\alpha}\right.$ and $\left.\mathrm{C}\right)$ and below $1.4 \AA$ for all heavy atoms. Side chains are well defined. It is evident that the ensemble of structures satisfies the experimental constraints and exhibits only small deviations from the idealized covalent geometry. Majority of the dihedral angles appear on the most-favored region of the Ramachandran plot. Table 2.2 also compares the FastNMR structure with conventionally determined structure. The manually and automatically determined structures were of similar energy. The FastNMR structures of Conk-S1 and Ubiquitin deviate by $0.4 \AA$ and $0.6 \AA$ from the conventionally determined NMR structures [Bayrhuber, Graf 2006;Bayrhuber, Vijayan 2005;Cornilescu, Marquardt 1998]. Figure 2.3 shows the direct comparison of the two FastNMR determined structure with the manually determined structures. The FastNMR calculation of each protein was completed in less than 24 hours. 


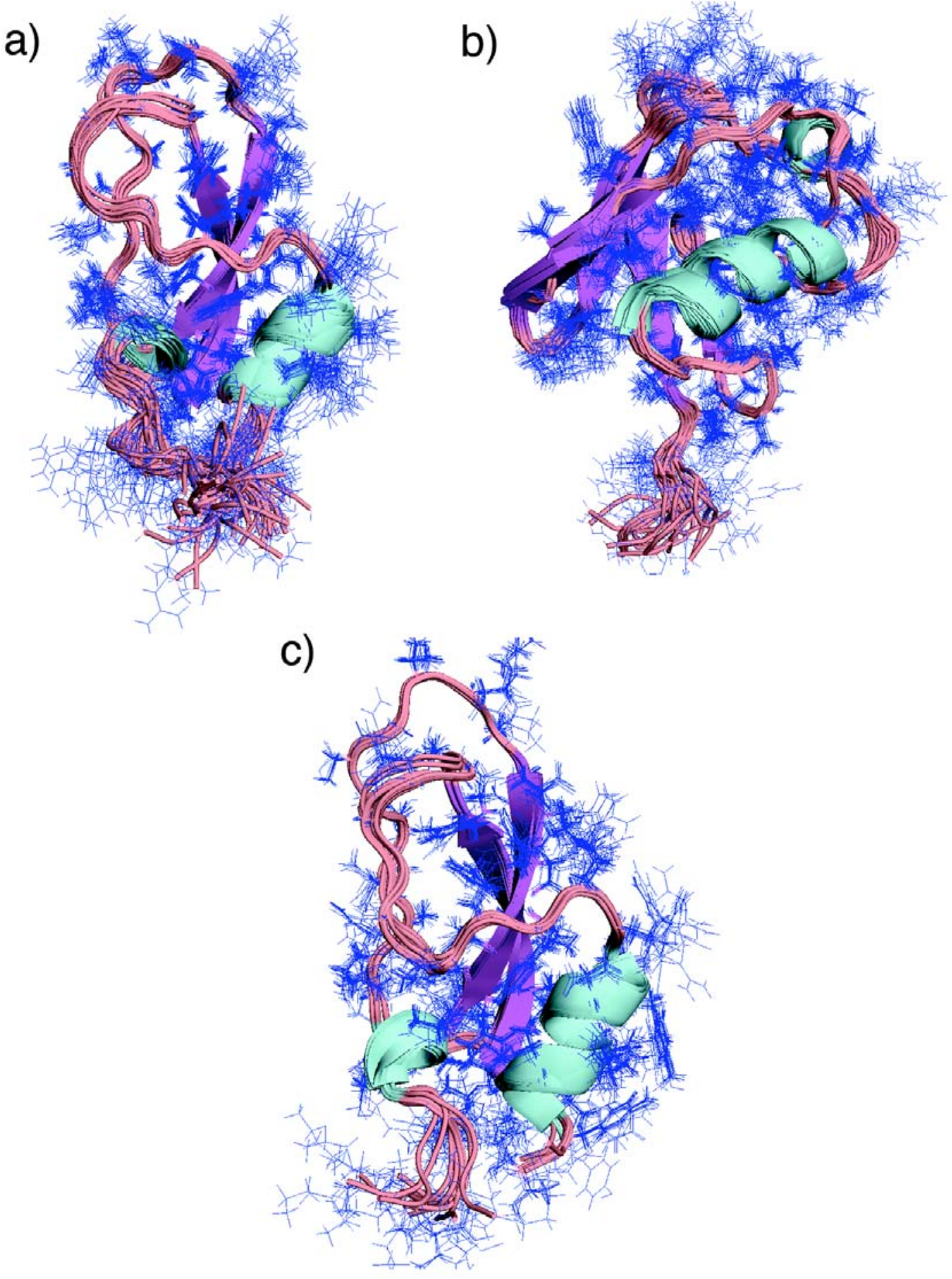

Figure 2.2: FastNMR 3D structure of a) Conk-S2, b) Ubiquitin, and c) Conk-S1. 

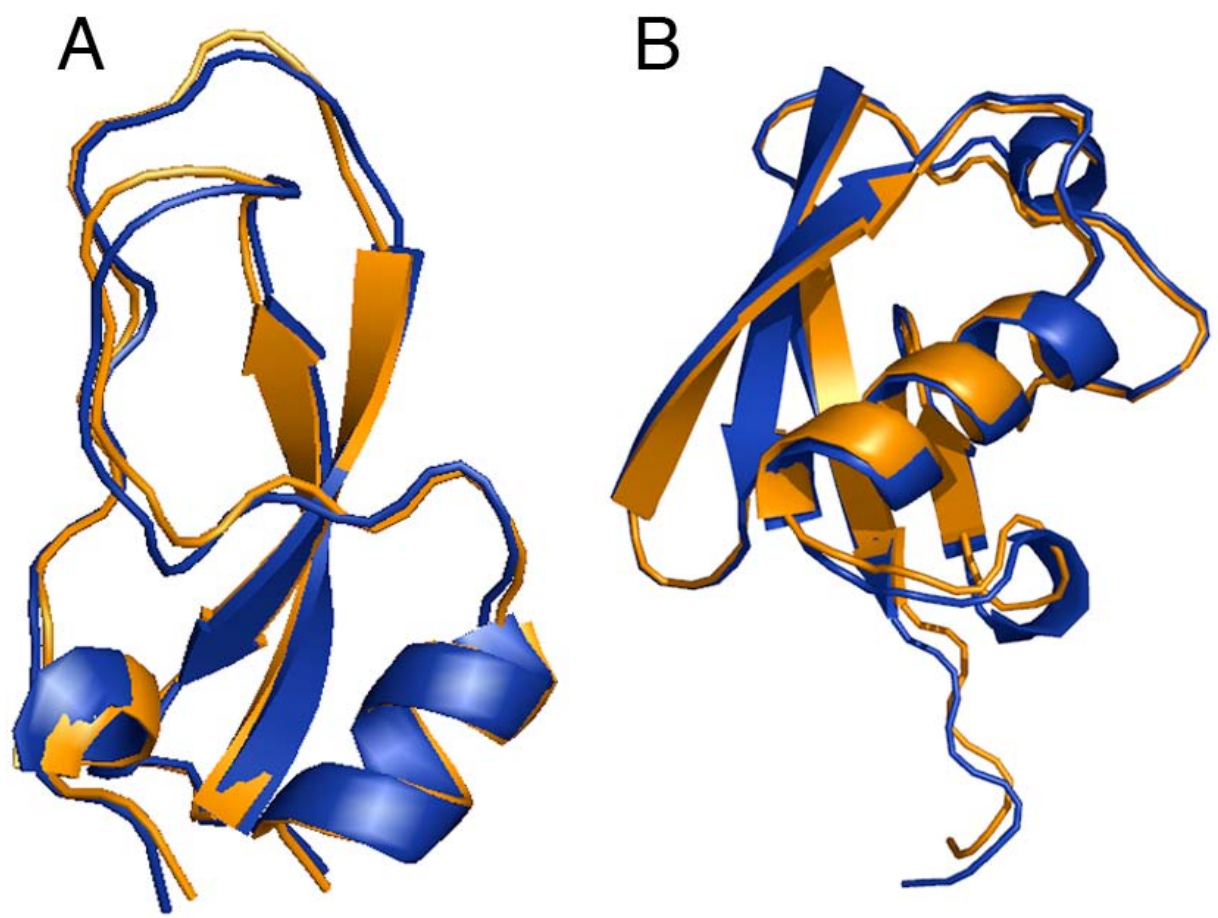

Figure 2.3: Comparison of FastNMR structures with structures deposited in the Protein Data Bank (PDB). (A) conk-S1. (B) Ubiquitin. FastNMR structures are shown in orange, structures deposited in the PDB (PDB codes: 1D3Z and 2CA7 for ubiquitin and Conk-S1, respectively) are shown in blue. 
Table 2.2. Structural statistical data of the investigated peptides. ${ }^{\text {[a] }}$

\begin{tabular}{|c|c|c|c|c|c|}
\hline & \multicolumn{2}{|c|}{ Conk-S1 } & \multicolumn{2}{|c|}{ Ubiquitin } & \multirow{2}{*}{$\begin{array}{l}\text { Conk-S2 } \\
\text { FastNMR }\end{array}$} \\
\hline & $2 \mathrm{CA} 7$ & FastNMR & $1 \mathrm{D} 3 \mathrm{Z}^{[\mathrm{b}]}$ & FastNMR & \\
\hline Number of NOEs ${ }^{[c]}$ & 551 & 464 & $1744^{[\mathrm{d}]}$ & 635 & 570 \\
\hline Long-range & 113 & 72 & 731 & 119 & 160 \\
\hline Medium-range & 73 & 57 & 291 & 106 & 79 \\
\hline Short-range & 365 & 335 & 722 & 410 & 331 \\
\hline Number of dihedral angles & 126 & 126 & 98 & 127 & 167 \\
\hline Violations $>5^{\circ}$ & $3 \pm 1$ & $3 \pm 1$ & 0 & $1 \pm 1$ & $2 \pm 1$ \\
\hline Number of RDCs & $190^{1}$ & $190^{1}$ & $200^{2}$ & $200^{2}$ & $138^{3}$ \\
\hline RDC types ${ }^{[\mathrm{e}]}$ & $1,2,3,4$ & $1,2,3,4$ & $1,2,3$ & $1,2,3$ & $1,2,3$ \\
\hline Energy $\left[\mathrm{kcal} \mathrm{mol}^{-1}\right]$ & -1267.2 & -1387.9 & -2767.9 & -2247.6 & -1025.0 \\
\hline $\begin{array}{l}\text { Most-favored } \\
\text { Disallowed }\end{array}$ & $\begin{array}{c}\text { Ramachan } \\
88.2 \\
2.0\end{array}$ & $\begin{array}{c}\text { dran plot [\%] } \\
87.5 \\
1.0\end{array}$ & $\begin{array}{r}95.0 \\
0.0\end{array}$ & $\begin{array}{r}95.5 \\
0.0\end{array}$ & $\begin{array}{c}84.5 \\
1.9\end{array}$ \\
\hline \multicolumn{6}{|c|}{ Coordinate $\operatorname{fr}_{\mathrm{f}]}$ ccision $[\AA]$} \\
\hline $\begin{array}{l}\text { Backbone atoms } \\
\text { All heavy atoms }\end{array}$ & $\begin{array}{l}0.6 \\
1.2\end{array}$ & $\begin{array}{l}0.7 \\
1.4\end{array}$ & $\begin{array}{l}0.3 \\
0.9\end{array}$ & $\begin{array}{l}0.4 \\
1.1\end{array}$ & $\begin{array}{l}0.6 \\
1.2\end{array}$ \\
\hline
\end{tabular}

[a] Statistics for ensembles of 20 structures. [b] Structure recalculated based on experimental restraints of 1D3Z. [c] None of the structures exhibited distance violations greater than $0.5 \AA$. [d] Only $58 \%$ of the long-range NOEs are non-redundant. [e] 1, 2, 3, 4 refer to ${ }^{1} \mathrm{D}_{\mathrm{N}-\mathrm{H}},{ }^{1} \mathrm{D}_{\mathrm{C}-\mathrm{N}},{ }^{1} \mathrm{D}_{\mathrm{C} \alpha-\mathrm{C}},{ }^{1} \mathrm{D}_{\mathrm{C} \alpha-\mathrm{H} \alpha}, \mathrm{RDCs}$, respectively. [1] 46, 50, 47 and 47 RDCs of types 1, 2, 3 and 4, respectively. [2] 69, 67 and 64 RDCs of types 1, 2 and 3, respectively. [3] 54, 54 and 30 RDCs of types 1, 2 and 3, respectively. [f] Defined as the average RMS difference between the final 20 FastNMR structures and the mean coordinates for residues 272 (ubiquitin), 3-60 (Conk-S1) and 5-60 (Conk-S2). 


\subsubsection{Backbone assignment and fold determination by FastNMR}

In case of Conk-S1, all 55 residues, for which signals in $2 \mathrm{D}{ }^{1} \mathrm{H} /{ }^{15} \mathrm{~N}$ correlation spectra were detected, had been correctly assigned at the end of stage A of FastNMR and nearly complete backbone assignment was obtained for Ubiquitin. With nearly complete backbone assignment and a large number of RDCs (190 and 200, respectively, for Conk-S1, Ubiquitin), low-resolution folds of both the proteins were obtained at the end of stage A.

\subsubsection{Side chain assignment and assignment analysis}

In FastNMR, the assignment of experimental side-chain resonances is performed automatically by comparison with values predicted from protein folds established at stage $\mathrm{A}$ in the FastNMR calculation. Assignments are considered only when the difference between predicted and measured chemical shift is less than $0.3 \mathrm{ppm}$ for protons and $1.3 \mathrm{ppm}$ for carbons. To justify the parameters, tests were done, on Ubiquitin, by predicting the chemical shifts from the protein fold obtained at the end of stage A of FastNMR, that are deviating by 2.4 and $3.5 \AA$ from the native structure, 1D3Z. The predicted values were compared to the experimental chemical shifts obtained from 3D HCCONH- and CCONH-TOCSY experiments recorded on the protein. These tests show that the root-mean-square deviation (rmsd) between predicted and experimental chemical shifts is $0.19 \mathrm{ppm}$ for protons and $1.1 \mathrm{ppm}$ for carbons visible in HCCONH- and CCONH-TOCSY spectra [Meiler 2003;Osapay and Case 1994] (Figure 2.4).

In addition, when two experimentally observed ${ }^{1} \mathrm{H}$ chemical shifts, which belong to the same residue (as established by HCCONH and CCONH-TOCSY spectra), differ by less than $0.3 \mathrm{ppm}$, any NOE to either of the two shifts is considered 

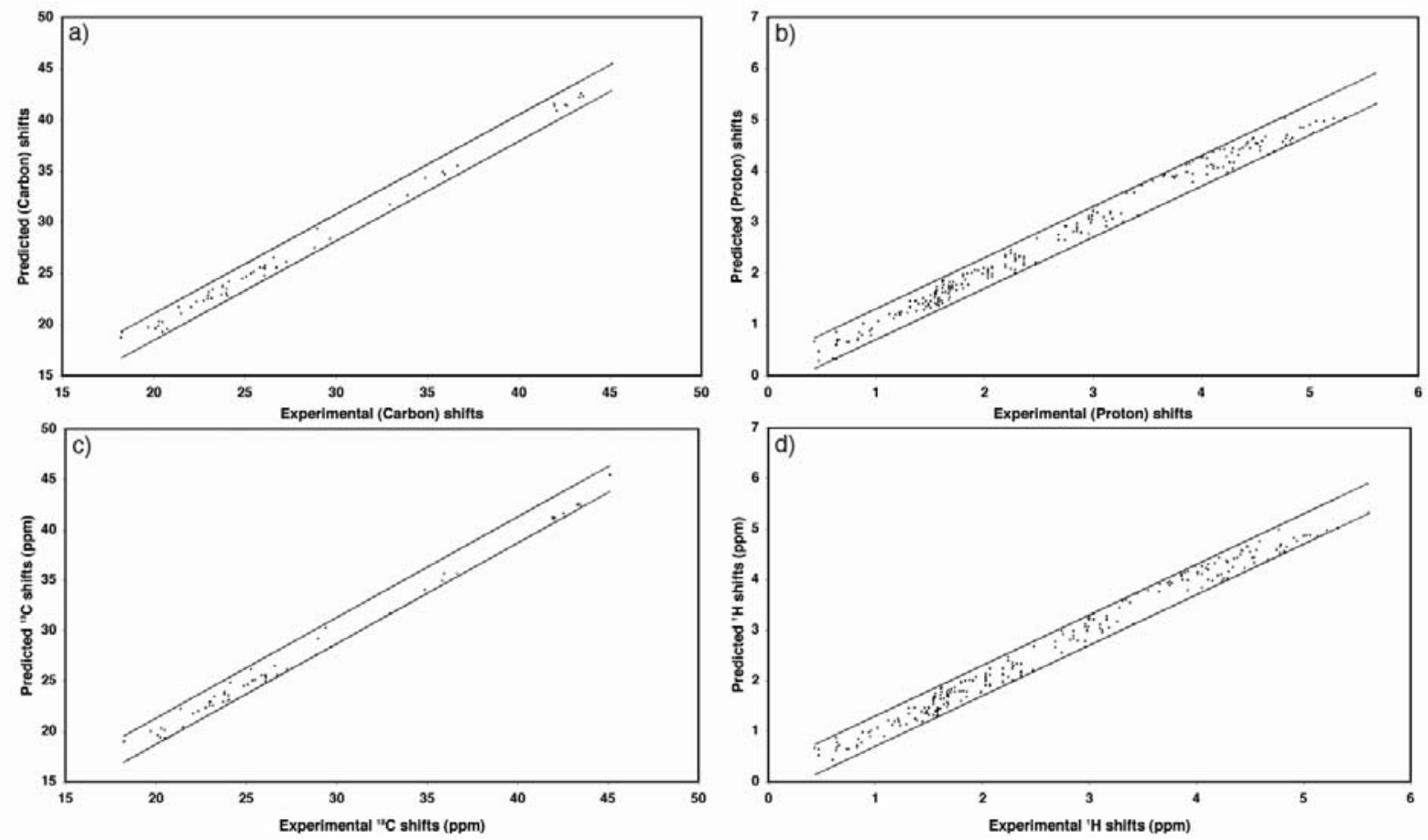

Figure 2.4: Comparison of predicted chemical shifts with experimental values obtained from 3D $\mathrm{HC}(\mathrm{CO}) \mathrm{NH}$ - and $\mathrm{C}(\mathrm{CO}) \mathrm{NH}-\mathrm{TOCSY}$ experiments recorded on ubiquitin. (A) ${ }^{13} \mathrm{C}$ chemical shifts were predicted using a artificial neural network as implemented in the software PROSHIFT [Meiler 2003] from a structure that deviates $2.4 \AA$ from the native structure (PDB code: $1 \mathrm{~d} 3 \mathrm{z}$ [Cornilescu, Marquardt 1998]). Note that the comparison does not include $\mathrm{C}_{\alpha}$ and $\mathrm{C}_{\beta}$ chemical shifts as these are assigned based on the chemical shift information obtained from backbone triple-resonance NMR experiments. (B) ${ }^{1} \mathrm{H}$ chemical shifts were predicted using empirical rules as implemented in the software SHIFTS [Osapay and Case 1994] from the same structure as in A). (C) Same as A) but using a structure that deviates $3.5 \AA$ from the $1 \mathrm{~d} 3 \mathrm{z}$ structure. (D) ${ }^{1} \mathrm{H}$ chemical shifts were predicted using empirical rules as implemented in the software SHIFTS from the same structure as in $\mathrm{C}$ ). The straight lines bracket the range $\pm 0.3 \mathrm{ppm}$ between measured and predicted ${ }^{1} \mathrm{H}$ chemical shifts and $\pm 1.3 \mathrm{ppm}$ between measured and predicted ${ }^{13} \mathrm{C}$ chemical shifts. 


\section{Table 2.3 NMR chemical shift assignments obtained by FastNMR}

\begin{tabular}{|c|c|c|c|c|c|c|c|}
\hline \multirow[t]{3}{*}{ Protein } & \multirow{3}{*}{$\begin{array}{c}\# \text { of } \\
\text { residues }\end{array}$} & \multirow{3}{*}{$\begin{array}{l}\text { Chemical } \\
\text { shifts }^{\text {a }}\end{array}$} & \multirow{3}{*}{$\begin{array}{c}\text { Backbone } \\
\text { assignment } \\
(\%)^{b}\end{array}$} & \multicolumn{4}{|c|}{ Side chain assignment $(\%)^{\mathrm{c}}$} \\
\hline & & & & \multicolumn{2}{|c|}{ Start $^{\mathrm{d}}$} & \multicolumn{2}{|c|}{ Final $^{\mathrm{e}}$} \\
\hline & & & & ${ }^{13} \mathrm{C}$ & ${ }^{1} \mathrm{H}$ & ${ }^{13} \mathrm{C}$ & ${ }^{1} \mathrm{H}$ \\
\hline Conk-S1 & 60 & $\mathrm{C}_{\alpha} / \mathrm{C}_{\beta}$ & $\begin{array}{l}100 \\
(97)\end{array}$ & $\begin{array}{r}100.0 / 0 \\
(70.0 / 0\end{array}$ & $\begin{array}{c}89.7 / 10.3 \\
62.8 / 7.2\end{array}$ & $\begin{array}{l}100.0 / 0 \\
70.0 / 0\end{array}$ & $\begin{array}{r}93.7 / 6.3 \\
65.6 / 4.4)\end{array}$ \\
\hline Ubiquitin & 76 & $\mathrm{C}_{\alpha} / \mathrm{C}_{\beta}$ & $\begin{array}{l}95 \\
(92)\end{array}$ & $\begin{array}{l}90.0 / 0 \\
(50.0 / 0\end{array}$ & $\begin{array}{l}88.5 / 11.5 \\
57.4 / 7.4\end{array}$ & $\begin{array}{c}90.0 / 0 \\
50.0 / 0\end{array}$ & $\begin{array}{l}96.7 / 3.3 \\
62.8 / 2.1)\end{array}$ \\
\hline
\end{tabular}

\footnotetext{
${ }^{\text {a }}$ Chemical shifts used to establish sequential connectivity.

b Percentage of assigned experimental spin systems. Assignment scores with respect to the total number of assignable residues are given in brackets.

${ }^{c}$ Percentage of measured side chain chemical shifts that were assigned by FastNMR. Unambiguous ("numerator") and ambiguous ("denominator") assignment scores are listed. Assignment scores with respect to the total number of side chain atoms are given in brackets.

${ }^{\mathrm{d}}$ Start: prior to starting the NOE analysis in stage B of FastNMR.

e Final: upon completion of FastNMR. As part of the iterative NOE assignment and structure calculation, the NOE assignment is selected that produces the best-fit between the experimental NOE distance restraints and those back-calculated from the structure. Thus, during stage B the number of unambiguously assigned proton chemical shifts was increased from 89.7 to $93.7 \%$ for Conk-S1 and from 88.5 to $96.7 \%$ for ubiquitin. The assignments are highly useful for further NMR studies, such as relaxation time measurements to probe backbone and side chain motions.
}

as ambiguous (indistinguishable assignment) during the automated NOE assignment.

By using this approach, all experimentally observed carbon chemical shifts of Conk-

$\mathrm{S} 1$, Conk-S2 and ubiquitin were assigned unambiguously. ${ }^{1} \mathrm{H}$ chemical shifts, however, are often degenerate and about $10 \%$ of the measured side chain ${ }^{1} \mathrm{H}$ chemical shifts could not be assigned unambiguously.

Previously, it was suggested that for successful automated NOE assignment at least $90 \%$ of all proton chemical shifts have to be assigned [Jee and Guntert 2003]. FastNMR in its current implementation, however, only uses 3D CCONH- and HCCONH-TOCSY NMR experiments and only $\sim 60 \%$ of all protons were assigned by FastNMR prior to starting the NOE analysis, as shown in Table 2.3. Hence, in an attempt to increase the number of chemical shifts, we used theoretical chemical shifts. So, for all protons (including side chains) for which no experimental chemical shifts 
were available in 3D HCCONH-TOCSY spectra, FastNMR uses predicted chemical shifts, obtained from SHIFTS, for automated NOE assignment. As the predicted chemical shifts are not very accurate, the window size that is used for matching NOEs to ${ }^{1} \mathrm{H}$ chemical shifts was increased from $0.05 \mathrm{ppm}$ to $0.3 \mathrm{ppm}$. In case of carbons, missing chemical shifts are not substituted by theoretical values to reduce the risk of wrong NOE assignments. In addition, NOE distance restraints assigned to protons with predicted chemical shifts are used in the final structure refinement only if the same proton (predicted chemical shift) is assigned to two or more NOE peaks and the experimental chemical shift of the two NOE peaks differ by less than $0.1 \mathrm{ppm}$. In combination with the backbone conformation that is already established prior to the NOE analysis, this allows the determination of high-resolution protein structures.

FastNMR was applied to NMR data of three proteins with all the inherent difficulties of peak overlap, missing backbone resonances, noise peaks and multiple conformations. NOE peaks with multiple chemical shift assignments are fully taken into account by the use of ambiguous distance constraints. Many different tests were performed to highlight the reliability of FastNMR and to show that FastNMR works for real experimental data. In addition, we tested the impact of lower data quality and wrong backbone assignments. These tests are explained in the following section in detail.

\subsubsection{FastNMR produces correct high-resolution structures}

For testing the robustness of FastNMR, the FastNMR structure determination of the 60-residue neurotoxin Conk-S1 was interrupted prior to entering the NOE analysis

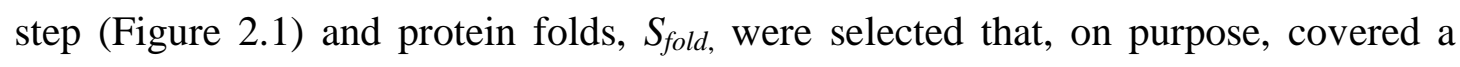

wide range of deviations $(\sim 2-7 \AA)$ from the native structure, $S_{\text {native }}$. These $S_{\text {fold }}$ conformations were fed into the following stages of the FastNMR calculation 
resulting in a large number of different ensembles, $S_{\text {final }}$, each containing 20 structures. The energy of these structures was compared to the rms (root-meansquare) deviation from the manually determined solution structure $S_{\text {native }}$ of Conk-S1 (Figure 2.5A). Two groups of structures were observed. In one group, the 20 lowestenergy structures didn't converge to the same fold or, despite they had the same fold, the conformation differed by more than $5 \AA$ from the native structure, i.e. $r m s\left(S_{\text {final }}\right.$ $S_{\text {native }}$ ) > $5 \AA$. The conformations belonging to this group did not properly fulfill all experimental restraints, as indicated by the very high energies. Structures in the second group had very low total energies and were very close to the manually determined NMR structure of Conk-S1.

\subsubsection{FastNMR tolerates missing and wrong backbone assignments}

With respect to backbone chemical shifts, the FastNMR structure of Conk-S1 (Figure 2.2C and Table 2.2) was determined using ${ }^{1} \mathrm{H}_{\mathrm{N}},{ }^{15} \mathrm{~N},{ }^{13} \mathrm{C}_{\alpha}(\mathrm{i}),{ }^{13} \mathrm{C}_{\alpha}(\mathrm{i}-1),{ }^{13} \mathrm{C}_{\beta}(\mathrm{i})$ and ${ }^{13} \mathrm{C}_{\beta}(\mathrm{i}-1)$ chemical shifts. These chemical shifts had been obtained from 3D HNCACB and a 3D CBCA(CO)NH experiment. To test the influence of lower data quality, the ${ }^{13} \mathrm{C}_{\beta}(\mathrm{i})$ and ${ }^{13} \mathrm{C}_{\beta}(\mathrm{i}-1)$ chemical shifts were removed from the input lists and FastNMR was rerun. This corresponds to the situation in which connectivity information is only available from a HNCA experiment. Actually, the situation is even more challenging, as ${ }^{13} \mathrm{C}_{\alpha}$ chemical shifts obtained from $\mathrm{HNCACB}$ and $\mathrm{CBCA}(\mathrm{CO}) \mathrm{NH}$ spectra are less accurate due to the lower resolution of these spectra compared to HNCA and $\mathrm{HN}(\mathrm{CO}) \mathrm{CA}$ spectra. Due to the lower quality of the input data, only $93 \%$ of all backbone signals (54 residues) were assigned prior to entering the NOE analysis, 


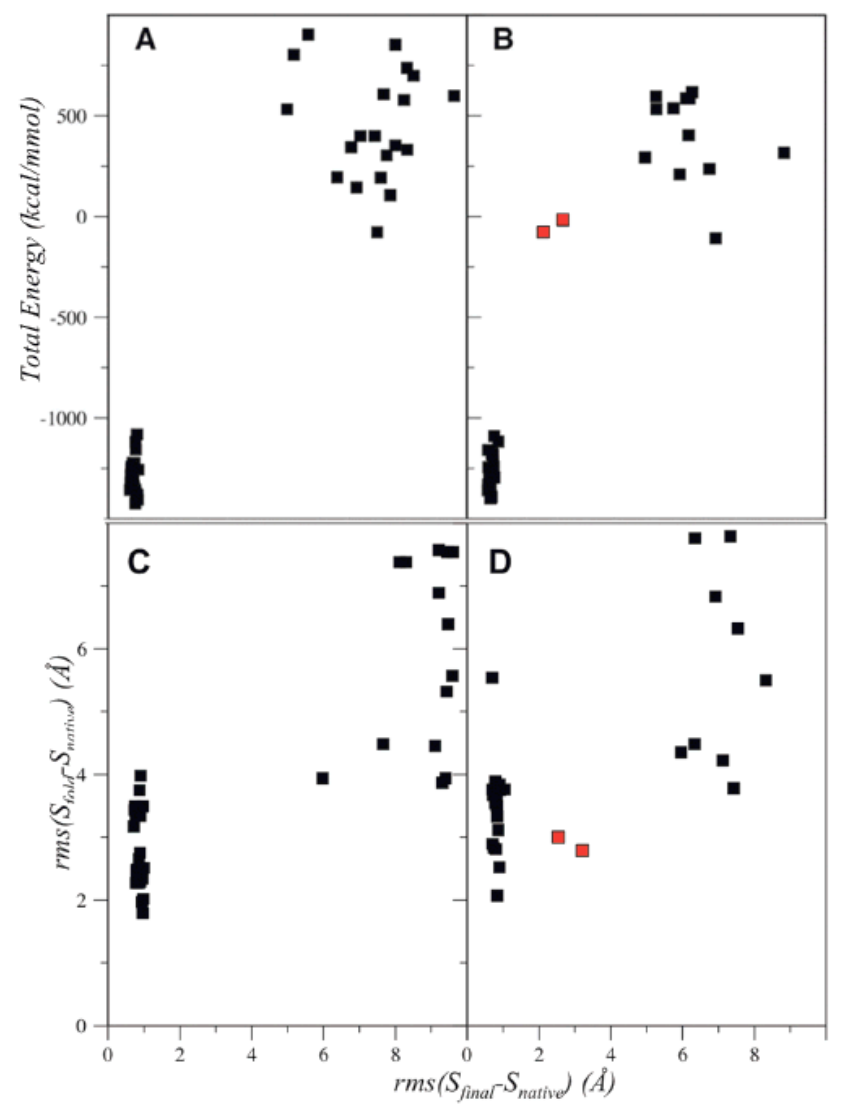

Figure 2.5: Stability tests of FastNMR performed on Conk-S1. In panels A and C, $97 \%$ of backbone resonances were assigned automatically by FastNMR (using $C_{\alpha}$ and $\mathrm{C}_{\beta}$ connectivity information and four types of RDCs) prior to entering the NOE analysis. After removing $\mathrm{C}_{\beta}$ connectivity information from the input to FastNMR, panels B and D, FastNMR obtained backbone assignments for only $93 \%$ of residues. In addition, we exchanged the assignment of two out of the $93 \%$ assigned residues on purpose, to probe the influence of wrong backbone assignments.

(A) and (B): Comparison of the energy of $S_{\text {final }}$ structures with the rms deviation of these structures from the conventionally determined solution structure (PDB code: 2CA7), rms $\left(S_{\text {final }}-S_{\text {native }}\right)$.

(C) and (D): Evaluation of the required accuracy of the protein fold, $S_{\text {fold }}$. The deviation of $S_{\text {final }}$ ensembles from the native structure (PDB code: $\left.2 \mathrm{CA} 7\right), \operatorname{rms}\left(S_{\text {final }}\right.$ $S_{\text {native }}$ ), was compared to the rms deviation of the native structure from the selected fold, $r m s\left(S_{\text {fold }}\right.$ - $\left.S_{\text {native }}\right)$. These data show that the deviation of the backbone-only conformation from the native structure, $\operatorname{rms}\left(S_{\text {fold. }}-S_{\text {native }}\right)$, has to be less than $3.5 \AA$ to achieve convergence to a high-resolution structure using FastNMR. Note that in two cases (marked red in panel D) FastNMR did not converge to a high-resolution structure despite the fact that the intermediate fold deviated by only $\sim 3 \AA$ from the native structure. Both of these problematic cases, however, are unambiguously identified as not converged by their high final energy (marked red in panel B).

whereas $97 \%$ had been assigned when $\mathrm{C}_{\beta}$ connectivity information was included.

Despite the missing backbone assignments, FastNMR produced a structure that was 
very close to the manually determined structure, with an rms deviation of $0.8 \AA$ for the backbone heavy atoms. With the reduced lists of backbone chemical shifts (without ${ }^{13} \mathrm{C}_{\beta}(\mathrm{i})$ and ${ }^{13} \mathrm{C}_{\beta}(\mathrm{i}-1)$ chemical shifts) the robustness tests described above were repeated, i.e. the FastNMR structure determination of the 60 -residue neurotoxin Conk-S1 was interrupted prior to entering the NOE analysis step, protein folds were selected that covered a wide range of deviations from the native structure, and the selected conformations were fed into the following stages of FastNMR. To make the tests even more demanding, the backbone assignment of two residues was interchanged, i.e. two residues were wrongly assigned on purpose. Nevertheless, all structures, which fulfilled the experimental restraints and had very low energies, deviated by less than $1.0 \AA$ from the native conformation (Figure 2.5B) (also refer text below Table 2.4).

\subsubsection{Influence of intermediate backbone conformation on convergence to a high-resolution structure}

How much is the intermediate backbone conformation (obtained at the end of stage A) allowed to deviate from the correct structure, such that FastNMR converges to a high-resolution structure? To address this question, we compared the deviation of the backbone conformations, selected in our robustness tests, from the native structure, $r m s\left(S_{\text {fold }}-S_{\text {native }}\right)$, with the deviation of the structures, obtained from these backbone conformations after completion of FastNMR, again from the native structure, $\operatorname{rms}\left(S_{\text {final }}-S_{\text {native }}\right)$. In all cases, FastNMR produced a high-resolution structure of Conk-S1 when the backbone conformation, prior to entering side chain chemical shift assignment, deviated by less than $\sim 3.5 \AA$ from the native structure (Figure $2.5 \mathrm{C}$ ). Whereas for $a b$ initio prediction this cannot generally be achieved, backbone conformations of domain-sized proteins obtained from NMR-based iterative backbone 
assignment and fold determination in most cases fulfill this requirement [Jung, Sharma 2004;Rohl and Baker 2002]. Note that no information about homology to proteins with known 3D structures is used in FastNMR, but could easily be incorporated.

Figure 2.5D shows the comparison of $r m s\left(S_{\text {fold }}-S_{\text {native }}\right)$ with $\operatorname{rms}\left(S_{\text {final }}-S_{\text {native }}\right)$ for the robustness tests, in which no $\mathrm{C}_{\beta}$ connectivity information was used. Despite the missing and two wrong backbone assignments, the convergence properties are very similar. When $r m s\left(S_{\text {fold }}-S_{\text {native }}\right)$ is smaller $\sim 3.5 \AA$, FastNMR converges to a highresolution structure of Conk-S1. Only in two cases (marked in red in Figure 2.5D) FastNMR did not converge to a high-resolution structure despite the fact that the intermediate fold deviated by only $\sim 3 \AA$ from the native structure. However, both these structures have very high energies (Figure 2.5B) clearly identifying them as not converged. Noteworthy in one case, FastNMR converged to a high-resolution structure (backbone rmsd of $0.7 \AA$ to the native structure) although the backbone conformation prior to side chain chemical shift assignment deviated by $5.8 \AA$ from the native structure (Figure 2.5D).

\subsubsection{FastNMR tolerates spurious peaks and multiple conformations}

In the various $3 \mathrm{D}$ experiments, in which the magnetization is detected on the ${ }^{1} \mathrm{H}_{\mathrm{N}}$ nuclei, one pair of ${ }^{1} \mathrm{H}_{\mathrm{N}}$ and ${ }^{15} \mathrm{~N}$ chemical shifts is expected for each amino acid in the protein except for prolines and the C-terminal residue. However, when parts of the protein exchange between different conformations, backbone signals can be missing if the exchange is intermediate on the NMR time scale, or additional signals can be present if the exchange is slow on the NMR time scale. Additional signals can also be due to spectral artifacts. 
The influence of multiple conformations and spurious peaks on the performance of FastNMR was tested for the 76-aa protein ubiquitin. The ten most Cterminal residues were removed from the primary sequence of ubiquitin, while keeping all 70 experimentally observed spin systems. Despite the presence of four spin systems, which did not correspond to any position in the primary sequence, FastNMR calculated a high-resolution structure of ubiquitin.

The tests on ubiquitin are strongly supported by the results obtained from the de novo FastNMR structure determination of the 65-aa toxin Conk-S2. Initially, Conk-S2 NMR samples were prepared in $50 \mathrm{mM}$ sodium acetate buffer, $\mathrm{pH}$ 5.2. In a first $2 \mathrm{D}{ }^{1} \mathrm{H}-{ }^{15} \mathrm{~N}$ HSQC recorded on Conk-S2, however, about 95 peaks potentially corresponding to ${ }^{1} \mathrm{H} /{ }^{15} \mathrm{~N}$ backbone nuclei were present. Therefore, the $\mathrm{pH}$ was raised to $\mathrm{pH} 6.3$ (50 mM sodium phosphate buffer). At $\mathrm{pH}$ 6.3, 60 backbone signals were visible in the $2 \mathrm{D}{ }^{1} \mathrm{H}_{-}{ }^{15} \mathrm{~N}$ HSQC spectrum and in the triple-resonance spectra, roughly fitting to the expected 59 backbone signals (Conk-S2 contains 5 prolines). The highresolution structure of Conk-S2 was then determined at pH 6.3 (see Fig. 1A and Table 1). FastNMR not only produced the high-resolution structure of Conk-S2, but also the assignment of backbone and side chain resonances. Surprisingly, only for 53 residues the backbone signals (and therefore also the side chain resonances) had been assigned by FastNMR, i.e. seven backbone signals remained unassigned. Therefore, additional triple-resonance experiments (3D $\mathrm{HNCO}, 3 \mathrm{D} \mathrm{HN}(\mathrm{CA}) \mathrm{CO}, 3 \mathrm{D} \mathrm{HNCA}$ and 3D $\mathrm{HN}(\mathrm{CO}) \mathrm{CA})$ were measured to see if the remaining seven ${ }^{1} \mathrm{H} /{ }^{15} \mathrm{~N}$ backbone signals could be assigned. Manual analysis of the six 3D triple resonance spectra finally proved that two of these signals correspond to V64 and G65. The others five spin systems, however, were multiple conformations of residues G65 (2x), V64 (1x), R6 (1x). One spin system could not be assigned. In addition, after close inspection of some weaker spin systems (not counted in the seven) were assigned to residues Q58, 
Q61 and Y62. No signals were observed for residues S8, G42, T53 and N54 due to chemical exchange intermediate on the NMR time scale. Thus, FastNMR was able to determine a high-resolution de novo structure of Conk-S2 despite significant complications due to chemical exchange. Despite these complications, FastNMR produced high-resolution structures, including the de novo structure of Conk-S2. These results demonstrate that FastNMR is highly robust.

Table 2.4 Deviation between different 3D structures of ubiquitin and crossvalidation by ${ }^{1} \mathrm{D}\left(\mathrm{C}^{\alpha} \mathrm{H}^{\alpha}\right)$ and ${ }^{1} \mathrm{D}\left(\mathrm{C}^{\alpha}, \mathrm{C}^{\beta}\right)$ RDCs. ${ }^{\mathrm{a}}$

\begin{tabular}{|c|c|c|c|c|}
\hline Structure & $1 \mathrm{ubq}^{\mathrm{b}}$ & $1 \mathrm{~d} 3 \mathrm{z}^{\mathrm{c}}$ & $1 \mathrm{~d} 3 \mathrm{z}$ refined ${ }^{\mathrm{d}}$ & FastNMR \\
\hline $1 \mathrm{ubq}$ & & 0.35 & 0.52 & 0.55 \\
\hline $1 \mathrm{~d} 3 \mathrm{z}$ & & & 0.45 & $0.56^{\mathrm{g}}$ \\
\hline $1 \mathrm{~d} 3 \mathrm{z}$ refined & 0.52 & 0.45 & & $0.41^{\mathrm{g}}$ \\
\hline FastNMR & 0.55 & 0.56 & 0.41 & \\
\hline $\begin{array}{l}\text { RDC cross- } \\
\text { validation }{ }^{e}\end{array}$ & 0.97 & $0.99^{f}$ & 0.98 & 0.97 \\
\hline
\end{tabular}

\footnotetext{
${ }^{a}$ Values given are rmsd values in $\AA$ and calculated for residues 2-72 between the mean structure of the NMR ensembles or the $1.8 \AA \mathrm{X}$-ray structure.

${ }^{\mathrm{b}} 1.8 \AA \mathrm{X}$-ray structure of ubiqutin (PDB code: $1 \mathrm{ubq}$ ).

${ }^{c}$ NMR solution structure of ubiquitin, which was determined with an extremely large number of experimental restraints, including six different types of RDCs (PDB code: 1d3z).

${ }^{\mathrm{d}}$ Structure of ubiquitin that was recalculated from the distance restraints, dihedral angles and three types of RDCs $\left({ }^{1} \mathrm{D}_{\mathrm{N}-\mathrm{H}},{ }^{1} \mathrm{D}_{\mathrm{C}-\mathrm{N}},{ }^{1} \mathrm{D}_{\mathrm{C} \alpha-\mathrm{C}}\right.$ ), which are available from the PDB (PDB code: $1 \mathrm{~d} 3 \mathrm{z} . \mathrm{mr}$ ).

${ }^{e}$ Pearson's correlation coefficient for the comparison of $62{ }^{1} \mathrm{D}_{\mathrm{C} \alpha-\mathrm{H} \alpha}$ and $39{ }^{1} \mathrm{D}_{\mathrm{C} \alpha-\mathrm{C} \beta} \mathrm{RDCs}$ with values back-calculated from the various structures using singular-value decomposition.

${ }^{\mathrm{f}}$ Note that the very high RDC correlation is due to the fact that the ${ }^{1} \mathrm{D}_{\mathrm{C} \alpha-\mathrm{H} \alpha}$ and ${ }^{1} \mathrm{D}_{\mathrm{C} \alpha-\mathrm{C} \beta}$ RDCs were used in the structure calculation of $1 \mathrm{~d} 3 \mathrm{z}$.

${ }^{g}$ Note that the rms deviation of the 20 lowest-energy structures of the FastNMR ensemble with respect to the average structure of this ensemble is $0.42 \AA$, whereas the maximum deviation of any of the 20 structures of the ensemble with respect to the average structure is $0.58 \AA$.
}

Cross-validation ensures that no wrong structures are produced by FastNMR: For backbone assignment and fold determination only RDCs and chemical shifts are used, whereas during automated NOE assignment RDCs are not used. Thus, in case the initial fold is incorrect it is unlikely that a sufficient number of NOEs is assigned during automated, structure-based NOE assignment. Even if a large enough number of NOEs is assigned, the NOE-based structure will likely differ significantly from the 
initial fold and disagree with the RDCs. Therefore, in the final stage of FastNMR when all experimental data are combined convergence to a low energy structure is not possible. This is clearly visible from Figure. $2.5 \mathrm{D}$ and the additional stability tests: Only for correct, high-resolution structures a low total energy is obtained by FastNMR. In addition, FastNMR structures have to pass the following check points: (i) at each stage during FastNMR structures must have converged to a unique conformation, (ii) structural changes during FastNMR must be less than $3.5 \AA$ from
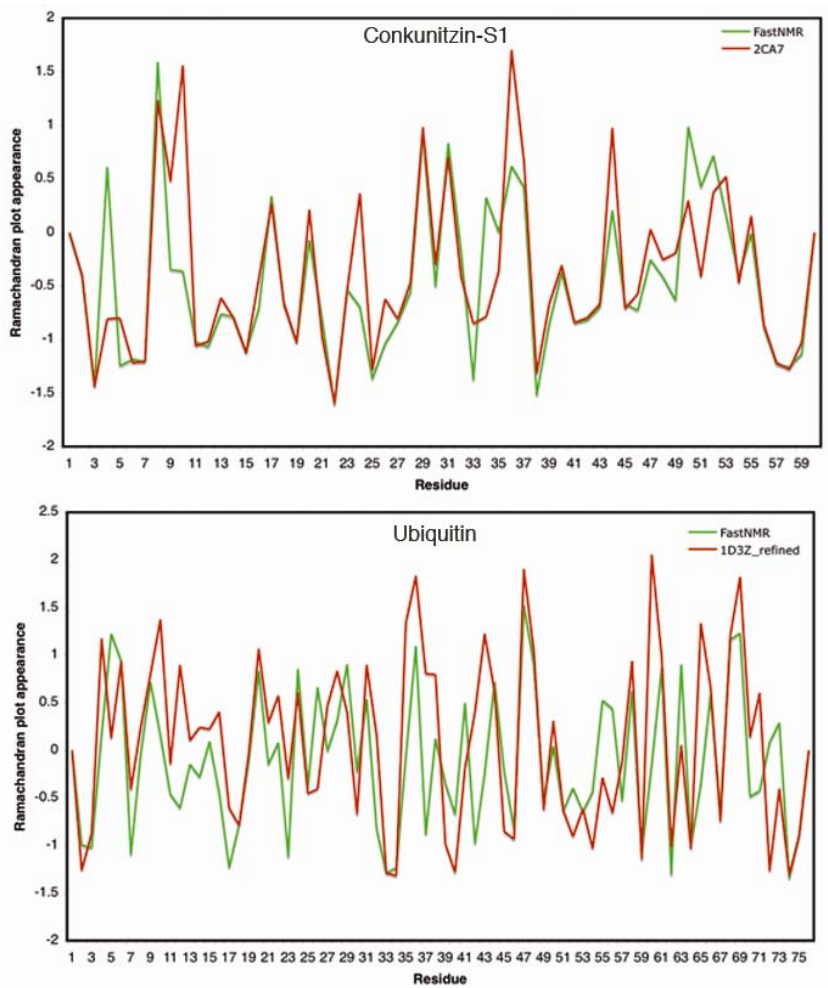

Figure 2.6: Comparison of per-residue Ramachandran plot quality Z-score between FastNMR structures and conventionally determined structures. (A) PDB (2CA7) structure (red) and FastNMR structure (green) of Conk-S1. (B) FastNMR structure of ubiquitin (green) and structure of ubiquitin recalculated from manually evaluated NMR data deposited in PDB (1D3Z.mr) (red). Average values for the twenty lowest-energy structures are shown. Additional structural statistics can be found in Table 1.1.

the initial fold to the high-resolution structure, (iii) more than $85 \%$ of the backbone resonances must have been assigned prior to starting the automated NOE assignment, 
and (iv) FastNMR structures have to pass the standard quality criteria such as violations of experimental restraints (Table 2.2). 


\subsection{Conclusions}

The strategy of FastNMR is based on the approach that has proven itself as robust in manual structure determination. This includes usage of information from tripleresonance experiments for sequential backbone assignment, use of iterative NOE assignment and structure calculation, and structure refinement using RDCs. Key to the success of FastNMR is, however, the simultaneous determination of the backbone assignment and the protein fold prior to analysis of NOE data.

FastNMR in its current implementation is limited to domain-sized proteins. This is mainly due to the fact that the only experiments which are used for extraction of side chain chemical shifts are CCONH- and HCCONH-TOCSY experiments. The performance of these experiments decreases with increasing molecular weight of the protein and they also do not allow access to chemical shifts of aromatic groups. A larger number of chemical shifts will be available, when 3D $(\mathrm{H}) \mathrm{CCH}-\mathrm{COSY}$ and 3D H(C)CH- TOCSY spectra [Kay, Xu 1993] are incorporated into FastNMR. In addition, aromatic chemical shifts can be obtained from two-dimensional $\left(\mathrm{H}_{\beta}\right) \mathrm{C}_{\beta}\left(\mathrm{C}_{\gamma} \mathrm{C}_{\delta}\right) \mathrm{H}_{\delta}$ and $\left(\mathrm{H}_{\beta}\right) \mathrm{C}_{\beta}\left(\mathrm{C}_{\gamma} \mathrm{C}_{\delta} \mathrm{C}_{\varepsilon}\right) \mathrm{H}_{\varepsilon}$ spectra [Yamazaki, Forman-Kay 1993]. The incorporation of these experiments into FastNMR is in progress.

In conclusion, we have demonstrated that it is possible to determine highresolution structures of domain-sized proteins within 24 hours starting from unassigned chemical shifts, RDCs and NOE peak lists and have used this approach to determine the de novo structure of Conk-S2. The calculation time can be reduced even further, when FastNMR is executed in parallel on several computers or when faster computers are available. FastNMR runs automatic, avoids wrong structures by crossvalidation, works for experimental data, only requires a limited number of NMR spectra and produces high-resolution structures. No manual assignment of chemical 
shifts or inter-residue correlations is required. Interactive work is confined to the processing of NMR spectra and to the preparation of input lists, which contain the unassigned, experimental chemical shifts, residual dipolar couplings and NOE peaks. As FastNMR is highly robust with respect to missing or wrong chemical shift assignments, we expect that it will be possible to perform peak picking and grouping also fully automatic.

FastNMR is a method for de novo structure determination, i.e. no prior structure or fold is assumed. However, with the rapid increase in the number of available 3D structures, it becomes more and more likely to find a close homologue in the Protein Data Bank (PDB) (www.rcsb.org). The structure of this homologue can be supplied as input to FastNMR. This will be important for larger proteins, for which the fold cannot be determined reliably using only RDCs and chemical shifts. When FastNMR is combined with methods for fast data acquisition, such as G-Matrix Fourier Transform NMR spectroscopy [Shen, Atreya 2005], it appears possible to obtain high-resolution NMR structures in less than one week after preparation of suitable NMR samples. Fast and efficient determination of high-resolution structures in solution will make biomolecular NMR a more efficient tool for Structural Biology. 


\section{Chapter 3}

\section{High-resolution 3D Structure Determination of Kaliotoxin by Solid-state NMR Spectroscopy}

\subsection{Introduction}

Structural characterization of membrane proteins and many other biological systems by X-ray crystallography or solution NMR spectroscopy is difficult because of problems with crystallization, solubility or molecular size. Significant advances, however, have been made to construct three-dimensional (3D) molecular structure from solid-state NMR data obtained under Magic Angle Spinning (MAS) [Andrew, Bradbury 1958] conditions [Griffin 1998;Luca, Heise 2003;Tycko 2001]. These efforts resulted in high-resolution 3D conformations for small peptides [Jaroniec, Macphee 2004;Nomura, Takegoshi 1999;Petkova, Ishii 2002;Rienstra, TuckerKellogg 2002] and the determination of medium-resolution backbone structures for a few solid-phase proteins [Castellani, Van Rossum 2002; Franks, Zhou 2005;Lange, Becker 2005;Zech, Wand 2005]. No high-resolution structure of a protein in the solidphase has been reported so far.

Recently, progress has been made to determine three-dimensional molecular structures existing in solid phase by magic angle spinning [Andronesi, Pfeifer 2004;Glaubitz and Watts 1998]. Unless sample orientation provides a direct route to monitor molecular structure under MAS conditions, the collection of medium and long-range distance constraints is most crucial. Two strategies have been developed in this direction: (i) measurement of ${ }^{13} \mathrm{C}-{ }^{13} \mathrm{C}$ distances on ${ }^{13} \mathrm{C}$ block-labeled protein microcrystals [Castellani, Van Rossum 2002] and (ii) extraction of ${ }^{1} \mathrm{H}-{ }^{1} \mathrm{H}$-distance restraints from ${ }^{13} \mathrm{C},{ }^{13} \mathrm{C}$ - and ${ }^{15} \mathrm{~N},{ }^{13} \mathrm{C}$-encoded ${ }^{1} \mathrm{H} /{ }^{1} \mathrm{H}$ mixing experiments on a 
uniformly ${ }^{13} \mathrm{C} /{ }^{15} \mathrm{~N}$-labeled sample [Lange, Becker 2005]. In the first approach, it uses proton-driven spin diffusion (PDSD) pulse sequence or dipolar assisted rotational resonance (DARR) [Takegoshi, Nakamura 2001] acquired ${ }^{13} \mathrm{C}-{ }^{13} \mathrm{C}$ distances and involves site directed isotope spin labeling, which leads to attenuation of the dipolar effects. Such approaches still depend on the extent of the labeling and require a high spectral dispersion to detect large number of long range ${ }^{13} \mathrm{C}-{ }^{13} \mathrm{C}$ correlations and the lack of it, leads to cross-peak overlaps. When spectral overlaps are present, manual assignment of the distance correlations becomes tedious, hence, requiring automatic cross-peak assignment. A recent approach, SOLARIA [Fossi, Castellani 2005], used solution-state method to overcome assignment of ambiguous PDSD cross-peaks and speed up structure calculation for solid phase proteins, thus proposing a method to automate the whole structure determination procedure. This approach though, is sensitive to the intermolecular peaks present in the peak list leading to local distortions in the structure.

Crucial to 3D structure determination is the detection of long-range distance correlations. Relayed magnetization transfer leads to the attenuation in the precision of correlations at long distances. Hence, additional difficulties in extraction of distance restraints from PDSD or DARR approach could be overcome by using single uniformly isotope labeled sample and from ${ }^{13} \mathrm{C},{ }^{13} \mathrm{C}$ encoded ${ }^{1} \mathrm{H},{ }^{1} \mathrm{H}$ mixing experiments (CHHC), an indirect detection of ${ }^{1} \mathrm{H}-{ }^{1} \mathrm{H}$ distances. In contrast to carbon correlations, all interactions in this spectra carries valuable structural information. This experiments allowed identifying a sufficient number of unambiguous long-range distance correlations, which defined the global 3D fold of KTX [Lange, Becker 2005]. Identifying an unambiguous cross peak for larger and complicated systems would be challenging in the presence of intermolecular peaks and wrong assignments. Here we combine this approach with a probabilistic assignment algorithm originally 
developed for the automatic assignment of ${ }^{1} \mathrm{H}-{ }^{1} \mathrm{H}$ correlations in Nuclear Overhauser Effect spectra recorded on proteins in solution [Kuszewski, Schwieters 2004]. We determine the high-resolution structure of the 38-residue scorpion toxin kaliotoxin (KTX) and show that the structure of KTX in the solid phase deviates from the one observed in solution. 


\subsection{Material and Methods}

We developed a strategy for rapid high-resolution structure determination from solidstate NMR (ssNMR) data obtained under the conditions of magic-angle spinning (MAS). In addition to the 3D structure of the protein, the method also yields crosspeak assignments of all spectra automatically. The strategy starts from a list of chemical shifts obtained from a series of two-dimensional ${ }^{13} \mathrm{C},{ }^{13} \mathrm{C}$ - and ${ }^{15} \mathrm{~N},{ }^{13} \mathrm{C}$ correlation experiments and peak lists from proton-proton correlated 2D $\mathrm{CHHC} / \mathrm{NHHC}$ spectra. The first step in this procedure is to assign the peak positions in the 2D N/CHHC spectra [Lange, Becker 2005] obtained at different mixing times. The probabilistic assignment algorithm for automated structure determination (PASD) [Kuszewski, Schwieters 2004] automatically performs this task by matching the assigned chemical shifts with the peak positions in 2D N/CHHC spectra. Earlier, the backbone fold of KTX (Protein Databank (PDB) code: 1XSW) [Lange, Becker 2005] in the solid phase was deduced from 28 manually assigned interresidue CHHC correlations using a conventional simulated annealing protocol. Building on the backbone fold and the few manual assignments of cross peaks, most of the peaks observed in 2D N/CHHC spectra were here assigned using PASD, a probabilistic assignment algorithm for automated structure determination. Subsequently, structure calculation was carried out in torsion angle space using the assigned distance restraints. Distance restraints were supplemented by torsion angle restraints derived

from ${ }^{1} \mathrm{H},{ }^{15} \mathrm{~N},{ }^{13} \mathrm{C}$ chemical shifts using the program TALOS [Cornilescu, Delaglio 1999]. Three successive PASD passes of cross peak assignment and simulated annealing were performed and each pass was started from a set of randomly generated coordinates. After completion of the PASD calculations, cross peak assignments were selected that had a likelihood of 1.0 (as defined by PASD). Selected assignments were 
then carefully verified by manual inspection of 2D CHHC and 2D NHHC spectra. In the final stage, manually verified cross peak assignments were used for calculation of a high-resolution structure using an optimized simulated annealing protocol [Linge, Williams 2003]. The calculations were started from random initial coordinates and all verified distance restraints were active during the course of calculation. In addition, torsion angle restraints derived from backbone chemical shifts were included.

PASD was applied largely following published procedures [Kuszewski, Schwieters 2004]. In short, three successive passes of simulated annealing calculations in torsion angle space were carried out. 500 independent structures were calculated for each pass. Each pass was started from a set of randomly generated coordinates. The target function comprised a potential function for experimental distance restraints (e.g. obtained from NOEs or CHHC correlations), a quadratic van der Waals repulsion term, a square-well potential for torsion angles and a torsion angle database potential of mean force. Pass 1 and 2 protocol comprised two hightemperature phases $(4000 \mathrm{~K}$ ) and a slow cooling phase (from 4000 to $100 \mathrm{~K}$ ) with a linear NOE potential. Pass 3 comprised a single high-temperature phase (4000 K) followed by a cooling phase with a quadratic NOE potential. Final assignment likelihoods were determined at the end of pass 3 calculations. Calculations were carried out on a Linux cluster of 32 processors and took about two days for each structure.

The method shares the same overall architecture of cross-peak assignment and structure calculation as in PASD. However, there are also significant differences. First, we have not used the ensemble of structures present at the end of pass 1 and 2 for calculation of likelihood estimates, but the high-resolution structure of KTX for solution-state structure, obtained using the same procedure. Second, since the final structure at the end of pass 3 do not represent fully-refined NMR structures, 
assignments of cross-peaks with a final likelihood of 1.0 were taken and verified by manual inspection of 2D CHHC and NHHC spectra. Manually verified cross-peaks were finally used for further refinement of the structures using an optimized simulated annealing protocol [Linge, Williams 2003; Schwieters, Kuszewski 2003]. The calculations were started from random initial coordinates and distance restraints were not randomly switched on and off during the calculation, but all verified restraints were active during the course of calculation.

At the end of pass 1 and 2 the PASD algorithm calculates likelihood estimates that each particular assignment associated with a cross-peak is correct. The likelihoods are calculated using the ensemble of structures present at the end of the corresponding pass. Thus, they are a metric of how consistent a given assignment is with the ensemble of structures at the end of each calculation pass. Here we have not used the ensemble of structures present at the end of pass 1 and 2 for calculation of likelihood estimates, but the high-resolution structures of KTX obtained under different conditions or the KTX fold determined previously. The structure calculation was done twice, in which the high-resolution structures of the solution state (obtained from the similar procedure from solution NMR data) and the solid-state was used for calculation of the likelihood estimates. For example, the structure calculations of KTX (solid phase) was done once by using KTX (solution state) for calculation of the likelihood estimates at the end of pass 1 and 2. Then a second calculation was done, in which the likelihood estimates were determined using KTX (solid phase). This approach was done iteratively for both the states. Thus, the structure calculations were biased on purpose toward the structure of the other state. In both the cases, the structures obtained for a specific state from the two different calculations were indistinguishable. Note, that identical structure calculation protocols were used in all cases. 


\subsection{Results and Discussions}

\subsubsection{High-resolution structure of KTX}

The approach was used to solve the high-resolution 3D structure of the 38-residue potassium channel blocker toxin KTX obtained after lyophilization and rehydration [Lange, Becker 2005]. ${ }^{1} \mathrm{H}-{ }^{1} \mathrm{H}$ correlations were detected in three $\mathrm{CHHC}$ spectra with mixing times of 250, 325 (Figure 3.1A) and $400 \mu \mathrm{s}$, and one NHHC spectrum (MAS rate of $12.5 \mathrm{kHz} ; \mathrm{B}_{0}=18.8 \mathrm{~T}$ ) [Lange, Becker 2005]. Proton-proton correlation 2D CHHC and 2D NHHC Spectra of the solid-state and 2D NOESY solution-state spectra was automatically peak picked using SPARKY 3 (T. D. Goddard and D. G. Kneller, SPARKY 3, University of California, San Francisco). Diagonal peaks were manually removed. Peak intensities obtained from the 2D CHHC and NHHC spectra were classified into four ranges and converted into distance ranges of 1.8-2.7, 1.8-3.3, 1.8-5.0 and 1.8-6.0 A, respectively. The classification was done independently for the four proton-proton correlation spectra. For analysis of the solid-state spectra by PASD, ${ }^{13} \mathrm{C}$ and ${ }^{15} \mathrm{~N}$ chemical shifts were labeled as if they were proton chemical shifts. The tolerances for matching chemical shifts to cross-peaks were set to 0.38 ppm in the acquisition dimension and to $0.60 \mathrm{ppm}$ in the indirect dimension. PASD structures do not represent fully-refined NMR structures [Kuszewski, Schwieters 2004]. Therefore, we selected cross-peaks with a final likelihood assignment (as defined by PASD) of 1.0. For KTX(solution) and KTX(solid), $31 \%$ and $28 \%$, respectively, of all long-range restraints, $83 \%$ and $80 \%$, respectively, of the mediumrange restraints, and $99 \%$ and $100 \%$, respectively, of the sequential restraints had final restraint likelihoods of 1.0. All other restraints had final restraint likelihoods of 0 . Assignments obtained for these cross-peaks by PASD were verified by manual inspection of the 2D CHHC spectra and the 2D NHHC spectrum. 
Previously, 15 long-range, 7 medium-range and 6 short-range correlations could be assigned manually using the same set of CHHC and NHHC spectra [Lange, Becker 2005]. Using the above-described semi-automated approach a total of $254{ }^{1} \mathrm{H}$ ${ }^{1} \mathrm{H}$ distance correlations could be assigned unambiguously (Figure 3.1A, 3.1B and Table 3.1). 64 of these were long-range, 37 medium-range and 153 sequential. The 3D solid-state structure of KTX that was calculated from the 254 distance restraints and 58 dihedral angle restraints is shown in Figure 3.2B (PDB code: 2UVS). The resulting ensemble of KTX structures tightly converged with a coordinate precision of $0.5 \AA$ and $1.4 \AA$ for backbone and side chain heavy atoms, respectively (Figure 3.3A). Backbone and most side chains had a well-defined orientation except the $\mathrm{N}$ - and $\mathrm{C}$ terminal residue and Asn30 located in the loop connecting the second and third $\beta$ strand of KTX. Weak correlations between RMSD of backbone and side chains may be arising from the side chain flexibility in free form of the structure. Overall, the structure showed a backbone fold consisting of a $\alpha$-helix affixed by disulphide bridges to a three-stranded $\beta$-sheet that is typical for the charybdotoxin family of $\mathrm{K}+$ channel-blocking scorpion toxins [Grissmer, Nguyen 1994]. 87\% of residues were found in the most favored region of the Ramachandran plot, whereas $3 \%$ of residues were found in the disallowed region. The high-resolution solid-state structure of KTX deviates by $2.4 \AA$ from the backbone conformation (PDB code: 1XSW) obtained on the basis of 28 manually assigned distance restraints [Lange, Becker 2005], which deviates by $2.7 \AA$ from the solution structure. The most pronounced deviation between 1XSW and the high-resolution structure was observed at the N-terminus, where four residues were rotated by about $50^{\circ}$, such that the first beta-strand was straight and not bent as seen in the high-resolution structure (in the solid phase or in solution). 

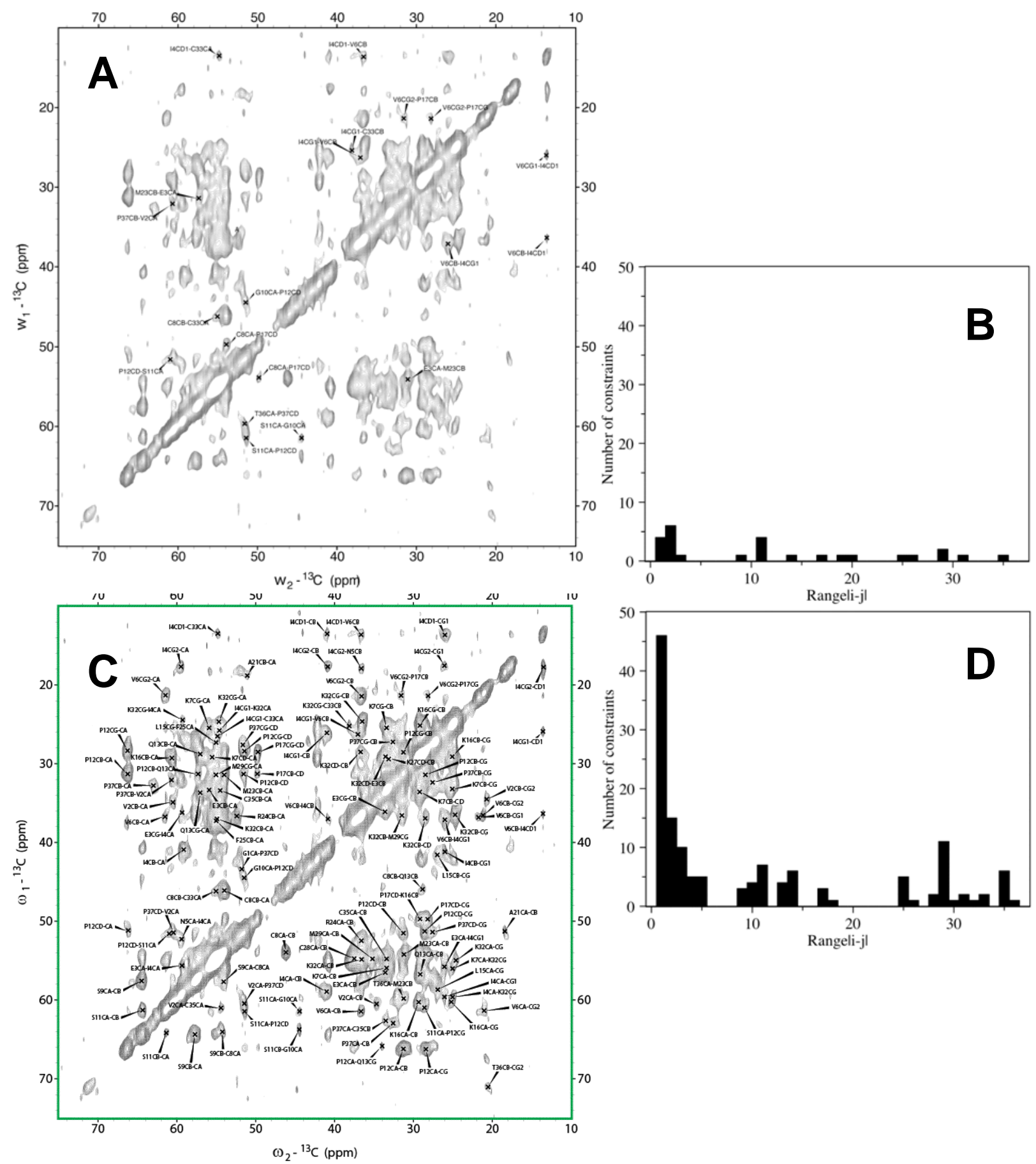

Figure 3.1: Comparison of interresidue correlations assigned manually (A and B) and ssigned unambiguously by PASD and verified manually (C and D) for KTX in the solid hase. Signals assigned in the 2D CHHC spectrum of $\mathrm{U}-\left[{ }^{13} \mathrm{C},{ }^{15} \mathrm{~N}\right]-\mathrm{KTX}$ recorded with a nixing time of $325 \mu$ s are labeled. Spectra in (A) and (C) are identical. (B) and (D) show he number of unambiguously assigned distance constraints as a function of residue lifference $i$ and $j$. 
Table 3.1: Structural statistics for the 20 lowest-energy structures of KTX in solution and in the solid phase.

\begin{tabular}{|c|c|c|}
\hline & Solution & Free \\
\hline \multicolumn{3}{|c|}{ Proton-Proton distance correlations } \\
\hline Total & 314 & 254 \\
\hline Short range & 199 & 153 \\
\hline Medium range & 45 & 37 \\
\hline Long range & 70 & 64 \\
\hline Distance violations $(>0.5 \AA)$ & 0 & $2^{b}$ \\
\hline Dihedral angles & 64 & 58 \\
\hline \multicolumn{3}{|l|}{ Energy (kcal/mol) } \\
\hline Total & -1203.32 & -1307.05 \\
\hline Bond & 34.02 & 32.49 \\
\hline Angle & 123.23 & 130.48 \\
\hline Improper & 36.77 & 35.04 \\
\hline Dihedral & 133.40 & 109.58 \\
\hline $\mathrm{NOE} / \mathrm{CHHC}$ & 166.82 & 156.58 \\
\hline \multicolumn{3}{|l|}{$\underline{\text { RMSD }}$} \\
\hline$\overline{\text { Bond }}$ & 0.0076 & 0.0074 \\
\hline Angles & 0.7832 & 0.8973 \\
\hline Improper & 0.8727 & 0.9013 \\
\hline Dihedral & 3.2033 & 4.7092 \\
\hline $\mathrm{NOE} / \mathrm{CHHC}$ & 0.0782 & 0.0791 \\
\hline \multicolumn{3}{|l|}{ Coordinate precision ${ }^{\text {a }}$} \\
\hline Backbone atoms $(\AA)$ & 0.7 & 0.5 \\
\hline All heavy atoms $(\AA)$ & 1.6 & 1.4 \\
\hline \multicolumn{3}{|l|}{ Ramachandran statistics } \\
\hline$\overline{\text { Most favored region }(\%)}$ & 86.7 & 84.0 \\
\hline Disallowed region (\%) & 3.3 & 3.7 \\
\hline
\end{tabular}

a Defined as the average rmsd difference between the 20 structures and the mean coordinates.

b The two distance restraints $\mathrm{E} 3(\mathrm{H} \alpha)-\mathrm{C} 33(\mathrm{H} \beta 1)$ and $\mathrm{C} 8(\mathrm{H} \beta 1)-\mathrm{C} 33(\mathrm{H} \alpha)$ were violated by 0.65 and $0.52 \AA$, respectively. The two restraints came from weak cross peak in the spectra and were assigned a distance range of $1.8-6.0 \AA$ in the calculations. 
A
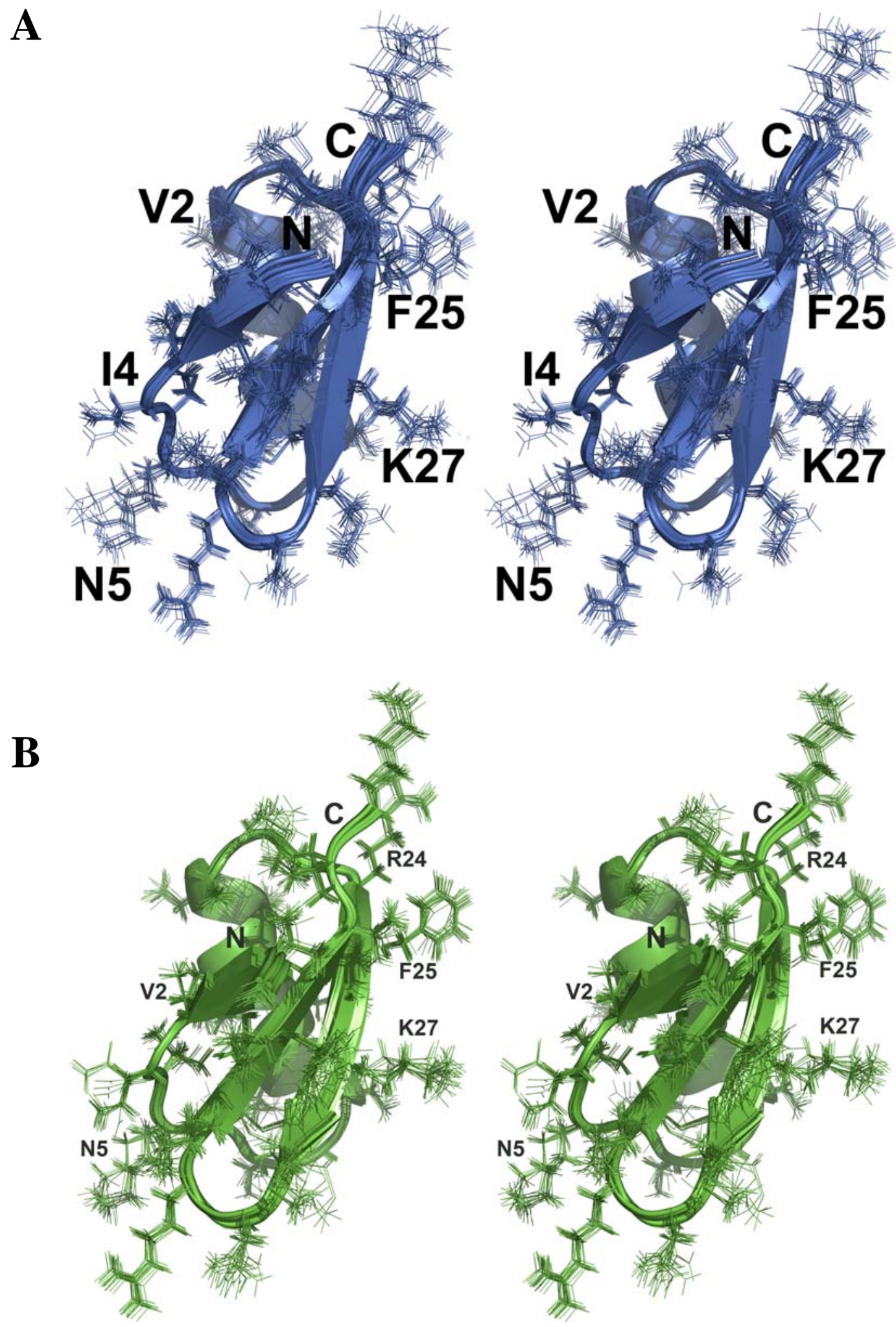

Figure 3.2: Stereo view of 20 lowest energy ensemble of high-resolution structures of Kaliotoxin in solution-state (A) (upper blue), and solid-state (B) (lower green) 


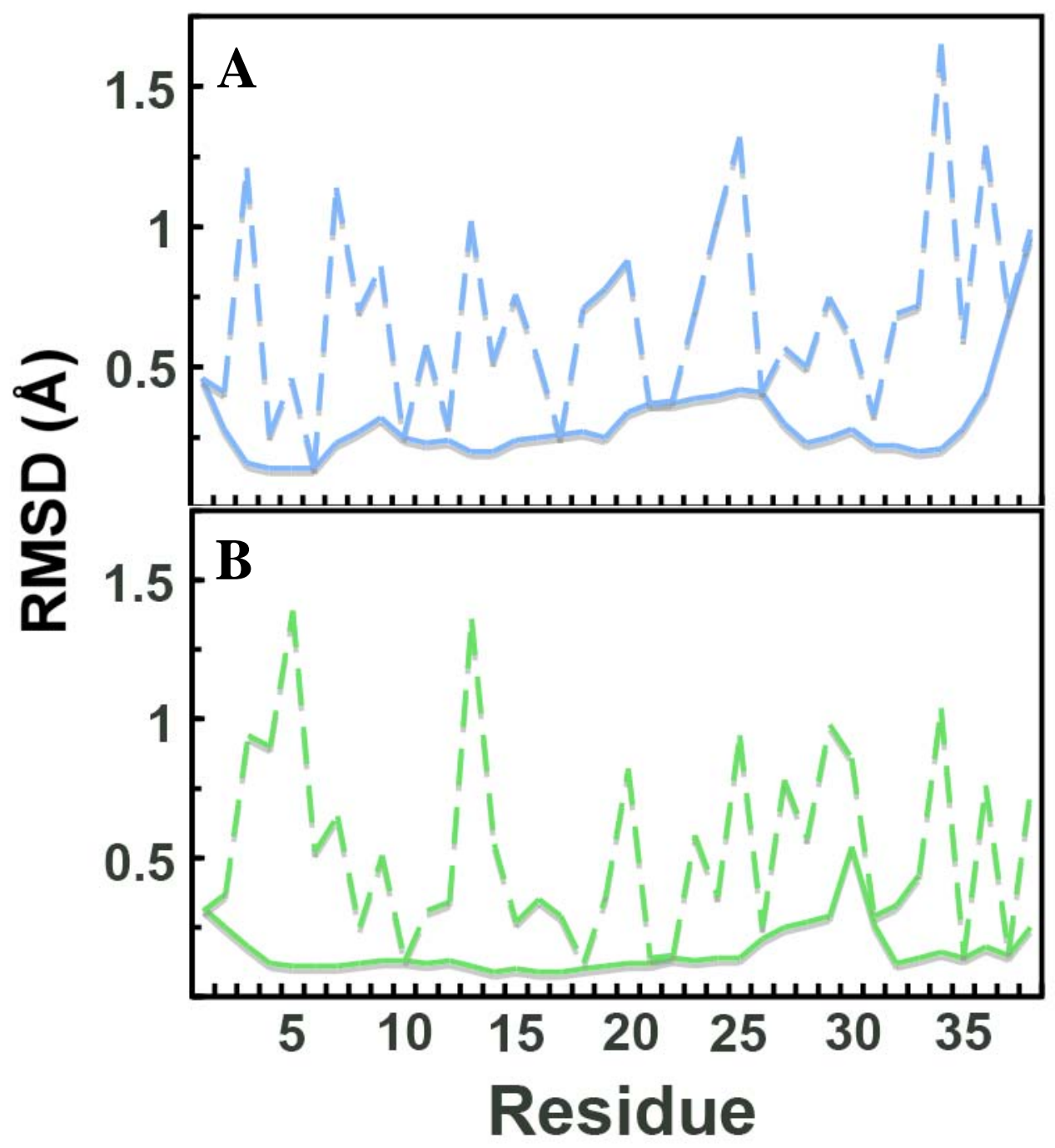

Figure 3.3: Coordinate precision of KTX in solution and solid-phase. Residue-based rms deviations of the atomic coordinates within the ensemble of 20 lowest energy structure of KTX in solution (A), in the solid phase (B). Deviations for the backbone atoms and for the non-hydrogen side chain atoms are shown as solid and dashed line, respectively. 

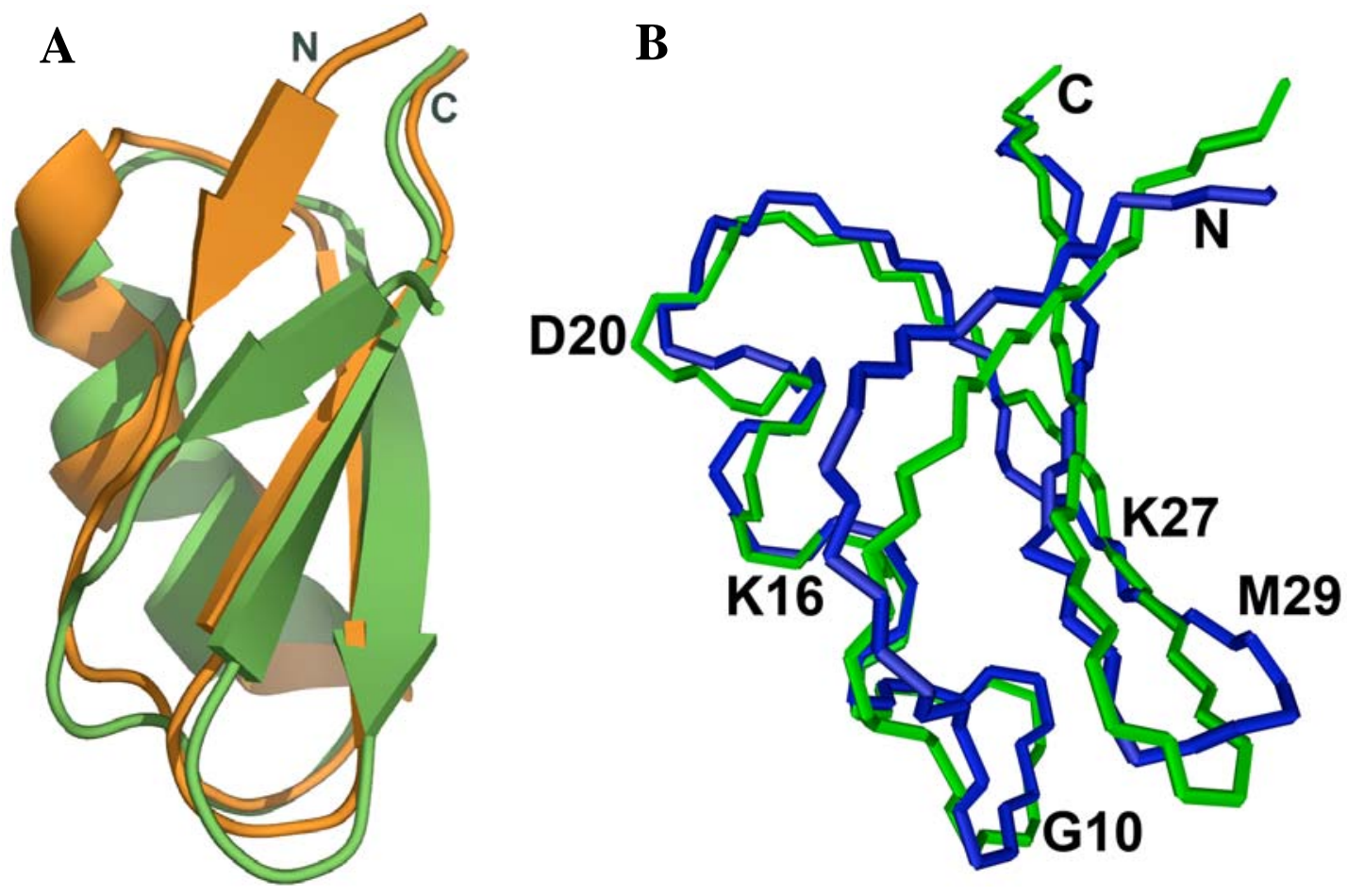

Figure 3.4: High-resolution solid-state structure of Kaliotoxin (green; determined in this study) compared to the backbone fold obtained previously from 28 manually assigned CHHC correlations (orange; PDB code: 1XSW) (Left). High-resolution solid-state structure of Kaliotoxin (green) compared to solution structure (blue; PDB code: 2KTX) (right).

To obtain convergence to a unique conformation, we fixed the 28 manually obtained CHHC cross peak assignments [Lange, Becker 2005]. Although some of the 28 distance restraints could be removed, a minimum of five long-range and two medium-range restraints were necessary to obtain convergence. In addition, the 1XSW backbone fold had to be used for calculation of PASD likelihood estimates to converge to a unique structure. Various additional tests were performed to probe the convergence of the structure calculations and support the accuracy of the highresolution solid-state structure (discussed below): (i) use of different conformations for calculating likelihood estimates in PASD; (ii) influence of chemical shift tolerances; (iii) sensitivity towards distance ranges used for interresidue correlations; (iv) dependence on the number of CHHC spectra; (v) influence of disulphide bond 
restraints. In all cases, the backbone of the calculated structures deviated by less than $0.7 \AA$ from the backbone of the structure shown in Figure 3.2B.

Recently, a method for automatic assignment of cross peaks in ${ }^{13} \mathrm{C}-{ }^{13} \mathrm{C}$ correlation spectra was developed [Fossi, Castellani 2005]. The approach called SOLARIA was used to analyze proton-driven spin diffusion (PDSD) spectra recorded on ${ }^{13} \mathrm{C}$ - block-labeled, microcrystalline preparations of the $\alpha$-spectrin $\mathrm{SH} 3$ domain. To attenuate dipolar truncation effects, the method requires different selectively ${ }^{13} \mathrm{C}$ enriched protein preparations. In addition, as cross peak volumes in PDSD spectra do not depend exclusively on the distance, a large distance boundaries had to be used in structure calculation. In that study, only a modest improvement in the 3D backbone structure was observed, and was also reported to be sensitive to the intermolecular peaks present in the peak list that lead to local distortions in the structure. Also, in such iterative approaches, if the initial assignment contains false constraints, the erroneous global fold of structures generated in the first calculation could bias the result of successive cycles. In this respect, the probabilistic algorithm, PASD [Kuszewski, Schwieters 2004], recently implemented in the structure determination package Xplor-NIH [Schwieters, Kuszewski 2003], provided a highly error tolerant approach for automated constraint identification and structure calculation cycles. Thus, no high-resolution structure of the backbone and the side chain of SH3 domain was reported. In contrast, our strategy based on C/NHHC correlations leads to an atomic resolution definition of both the backbone and the side-chain structure of KTX. We attribute these improvements to the higher fraction of long-range contacts in initial-rate N/CHHC spectra that allows for the same small distances boundaries [Baldus 2007;Lange, Seidel 2003] during structure calculation as used in liquid-state NMR. 


\subsubsection{Comparison with high-resolution solution-state NMR structure of KTX}

The 3D structure of KTX in solution had been previously determined by liquid state NMR spectroscopy. To enable a direct comparison with the structure of KTX in solid phase, we determined the solution structure of KTX employing the identical strategy as used for KTX in the solid phase. Tolerances for matching chemical shifts to cross-peaks were $0.015 \mathrm{ppm}$ in direct and indirect dimensions of the 2D NOESY spectrum. After completion of PASD calculations, cross peak assignments were selected that had a likelihood of 1.0. Selected assignments were carefully verified by manual inspection of the 2D NOESY spectrum. 70 long-range, 45 medium-range and 199 sequential NOEs could be assigned unambiguously, closely resembling the amount and distribution of distance restraints obtained from 2D N/CHHC spectra for

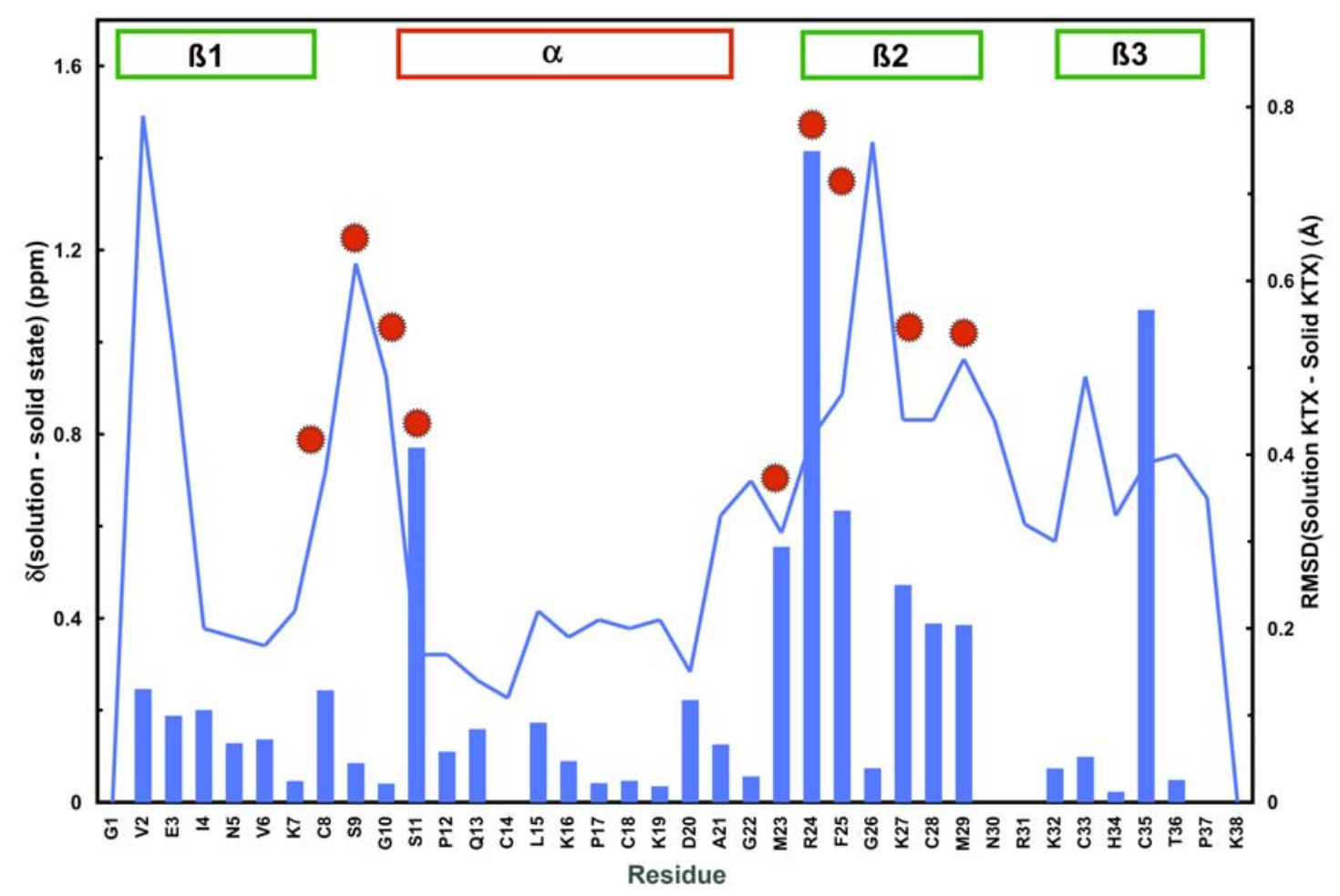

Figure 3.5: Comparison of averaged $\mathrm{C} \alpha / \mathrm{C} \beta$ chemical shift differences (blue bars; calculated according to $0.256 *\left[\Delta \delta C_{\alpha}^{2}+\Delta \delta C_{\beta}^{2}\right]^{1 / 2}$ ) with rms deviation between the mean structures (blue line) of KTX in solution and in the solid-phase. Red dots mark residues, for which the backbone dihedral angles predicted by TALOS differ in solution and the solid state. 


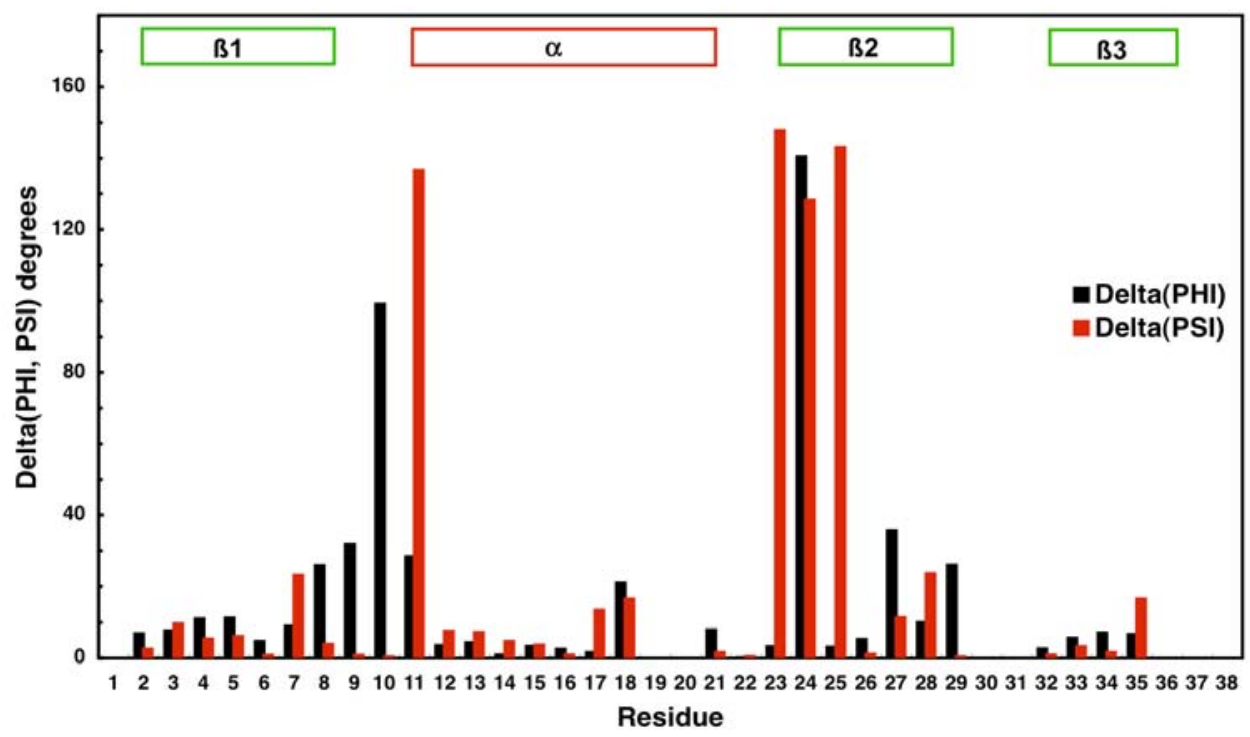

Figure 3.6: Differences between the backbone dihedral angles predicted by TALOS from the solution-state and solid-state secondary chemical shifts. The average uncertainty of TALOS prediction as stated on the web page is $\sim 12^{\circ}$. In Figure 3.5, residues were marked with a red dot, for which the difference in either phi or psi exceeds twice this value.

KTX in the solid phase (Table 3.1). The newly determined solution structure deviates by $0.6 \AA$ from a previously determined solution structure of KTX (PDB code: 2 KTX) [Gairi, Romi 1997]. The backbone of the high-resolution solid-state structure of KTX deviates by $1.3 \AA$ from that observed in solution (Figure 3.4B). Structural differences between the KTX (as seen from the pairwise root mean square deviation between the high-resolution structures of KTX), existing in solution and solid phase, were observed for the two N-terminal residues, the loop between the first beta-strand and the $\alpha$-helix, and the C-terminal beta-sheet in particular next to G26 (Figure 3.5).

The averaged $C \alpha / C \beta$ chemical shift differences between the solution and solid phase, in the above-mentioned regions in the structure, also supports this finding. The structural differences are due to a combination of changes in interresidue cross peaks and in backbone dihedral angles (Figure 3.6). For residues 8-11, 23-25, K27 and M29, backbone dihedral angles predicted by TALOS from the solid-state secondary 
chemical shifts clearly deviated from those predicted by TALOS from the solutionstate secondary chemical shifts (Figure 3.6).

\subsubsection{Reliability of High-resolution solid-state structure of KTX}

To check the reliability of the high-resolution solid-state structure and to account for the structural difference we performed several tests.

(i) Use of different structures for calculating likelihood estimates in PASD: biasing on purpose the calculation towards the solution-state structure.

At the end of pass 1 and 2 the PASD algorithm calculates likelihood estimates that each particular assignment associated with a cross-peak is correct. The likelihoods are calculated using the ensemble of structures present at the end of the corresponding pass. Thus, they are a metric of how consistent a given assignment is with the ensemble of structures at the end of each calculation pass [Kuszewski, Schwieters 2004]. Here we have not used the ensemble of structures present at the end of pass 1 and 2 for calculation of likelihood estimates, but either the high-resolution structure of KTX obtained under different conditions or a medium-resolution backbone fold. This improved convergence in the structure calculations and was justified as we previously established that the fold of KTX in solution and in the solid phase is the same [Lange, Becker 2005].

For example, the PASD calculations of KTX(solid) were done once by using KTX(solution) (PDB CODE: 2KTX) for calculation of the likelihood estimates at the end of pass 1 and 2. Thus, we biased on purpose the calculation towards the solutionstate structure. Then a second PASD calculation was done, in which the likelihood estimates were determined using the medium resolution backbone fold obtained previously for KTX in the solid phase (PDB CODE: 1XSW) [Lange, Becker 2005]. In all cases, the structures obtained from the two different PASD calculations were 
indistinguishable. This supports the relevance of the differences between the solution and solid-state structure. Note, that identical structure calculation protocols were used in all cases.

(ii) Influence of chemical shift tolerances.

For the calculations reported in the main part of the manuscript, tolerances for matching chemical shifts to cross-peaks were set to $0.38 \mathrm{ppm}$ and $0.60 \mathrm{ppm}$ in the acquisition and indirect dimension, respectively. We repeated the structure calculations with chemical shift tolerances of $0.38 \mathrm{ppm}$ and $0.4 \mathrm{ppm}$ in the acquisition and indirect dimension, respectively. The resulting structure deviated by less than 0.7 $\AA$ (rms value for all N, C $\alpha, \mathrm{CO}$ backbone atoms) from the structure shown in Figure 3.2B.

(iii) Influence of distance ranges: using only a single distance range $(1.8-6.0 \AA$ ).

Peak intensities obtained from the 2D CHHC and NHHC spectra were classified into four ranges and converted into distance ranges of 1.8-2.7, 1.8-3.3, 1.85.0 and 1.8-6.0 A, respectively. The classification was done independently for the four proton-proton correlation spectra (see Results and Discussion). To test the sensitivity of the solid-state structure to the used distance ranges, we repeated the structure calculations assigning to all N/CHHC correlations a distance range of 2.4$6.0 \AA$. The resulting structure deviated by less than $0.3 \AA$ (rms value for all $\mathrm{N}, \mathrm{C} \alpha$, $\mathrm{CO}$ backbone atoms) from the structure shown in Figure 3.2B.

(iv) Influence of the available spectra: usage of only two CHHC spectra instead of all four N/CHHC spectra.

The structure reported in the manuscript was calculated from distance restraints that were extracted from three CHHC spectra (mixing times of $250 \mu \mathrm{s}$, 
$325 \mu$ s and $400 \mu \mathrm{s})$ and one NHHC spectrum. When we only used the peak lists from the 250 and $400 \mu$ s CHHC spectrum, the number of long-range, medium-range and short-range correlations was reduced to 59, 33 and 102, respectively. Nevertheless, the 3D structure of $\mathrm{KTX}$ (solid) was highly similar to the one obtained with all four proton-proton correlation spectra (backbone rms deviation of $0.65 \AA$ ).

(v) Influence of disulphide bond restraints.

For both $\mathrm{KTX}$ (solution) and $\mathrm{KTX}$ (solid), structure calculations were performed without and with restraints for the three-disulphide bonds. The resulting structures did not differ (backbone rms deviation below $0.5 \AA$ ) and only the results of calculations, in which the disulphide bonds were not enforced, were reported.

(vi) Combining solid-state distance restraints with solution-state dihedral angles (and vice versa).

Are the structural differences due to an uncertainty in the analysis of N/CHHC spectra? To address this question, we recalculated the structure (using XPLOR-NIH and starting from an extended strand) using the same solid-state N/CHHC distance restraints, but supplementing them with the dihedral angles obtained by TALOS from the solution-state chemical shifts (instead of those obtained from the solid-state chemical shifts). The backbone of the resulting structure deviated by $0.5 \AA$ from the high-resolution solid-state structure. The coordinate precision for backbone and all heavy atoms was $0.7 \AA$ and $1.7 \AA$, respectively (Table 3.2). However, two dihedral angle violations were introduced (for residues 2 and 24) and residue 24 moved into the disallowed region of the Ramachandran plot. In addition, the total energy increased from $-1307 \pm 54 \mathrm{kcal} / \mathrm{mol}$ to $-1032 \pm 48 \mathrm{kcal} / \mathrm{mol}$, the dihedral angle energy from $-110 \pm 6 \mathrm{kcal} / \mathrm{mol}$ to $-16 \pm 36 \mathrm{kcal} / \mathrm{mol}$ and the distance restraint energy from - 
$157 \pm 28 \mathrm{kcal} / \mathrm{mol}$ to $-18 \pm 67 \mathrm{kcal} / \mathrm{mol}$ (when compared to the pure solid-state structure calculation).

Table 3.2: Structural statistics for the 20-lowest energy structures of KTX in solution and solid-phase, for test case $v i$, when combining the solid-state distance with solution-state backbone dihedral angles (and vice versa).

\begin{tabular}{lcc}
\hline & Solution & Free \\
\hline Proton-Proton distance correlations & 314 & 254 \\
Total & 199 & 153 \\
Short range & 45 & 37 \\
Medium range & 70 & 64 \\
Long range & 1 & 1 \\
Distance violations $(>0.5 \AA)$ & 64 & 58 \\
Dihedral angles & & 2 \\
Dihedral violations $\left(>5^{\circ}\right)$ & & \\
Energy (kcal/mol) & $-1154.5 \pm 65.3$ & $-1031.6 \pm 48.5$ \\
Total & $-34.6 \pm 5.8$ & $-17.7 \pm 5.5$ \\
Bond & $-105.4 \pm 35.1$ & $-75.1 \pm 36.2$ \\
Angle & $-36.77 \pm 11.2$ & $-35.04 \pm 14.6$ \\
Improper & $-54.6 \pm 23.2$ & $-16.3 \pm 36.4$ \\
Dihedral & $-30.6 \pm 52.9$ & $-17.9 \pm 66.7$ \\
NOE/CHHC & & \\
Coordinate precision & \\
Backbone atoms $(\AA)_{\text {All heavy atoms }(\AA)}^{\text {Ramachandran statistics }}$ & 0.7 & 0.5 \\
Most favored region $(\%)_{\text {Disallowed region }(\%)}$ & 1.6 & 1.4 \\
\hline a Defined as the average rmsd difference between & 20 structures and the mean coordinates. \\
& & \\
\hline
\end{tabular}

Similarly, when the solution-state distance restraints were combined with the solid-state dihedral angles, one dihedral angle violation (for S9) was introduced, the total energy was increased from $1203 \pm 60 \mathrm{kcal} / \mathrm{mol}$ to $-1154 \pm 65 \mathrm{kcal} / \mathrm{mol}$, the dihedral angle energy from $-133 \pm 6 \mathrm{kcal} / \mathrm{mol}$ to $-55 \pm 32 \mathrm{kcal} / \mathrm{mol}$ and the distance restraint energy from $-167 \pm 34 \mathrm{kcal} / \mathrm{mol}$ to $-31 \pm 53 \mathrm{kcal} / \mathrm{mol}$ (when compared to the pure solution-state structure calculation). The backbone of the resulting structure deviated 
by $0.6 \AA$ from the high-resolution solution-state structure. The coordinate precision for backbone and all heavy atoms was $0.8 \AA$ and $1.9 \AA$, respectively. These data demonstrate that the solid-state distance restraints are only in agreement with the solid-state backbone chemical shifts, and the solution-state distance restraints are only in agreement with the solution-state backbone chemical shifts. 


\subsection{Conclusions}

This study demonstrates that high-resolution 3D structures of globular proteins can be obtained from solid-state NMR data. The combination of ${ }^{15} \mathrm{~N},{ }^{13} \mathrm{C}$-encoded ${ }^{1} \mathrm{H} /{ }^{1} \mathrm{H}$ mixing experiments with a probabilistic cross peak assignment algorithm is particularly powerful, as short distances between protons provide the principal source of long-range structural information. Depending on the molecule under investigation, the presented approach can be combined with other solid-state NMR spectroscopic methods. Applications to larger proteins may benefit from the use of block [Castellani, Van Rossum 2002], modular [Pickford and Campbell 2004] or stereoarray [Kainosho, Torizawa 2006] isotope labeling schemes, and/or acquisition of higher-dimensional spectra and will therefore extend the applicability of this method in the determination of high-resolution structures of amyloid or membrane proteins and more complex systems. 


\section{Chapter 4}

\section{High-resolution 3D Structure of Kaliotoxin bound to the Potassium channel and toxin-channel complex structural model determined by Solid-state NMR Spectroscopy}

\subsection{Introduction}

Interactions between voltage-dependent potassium $(\mathrm{Kv})$ channels and peptide toxins from scorpion venom are among the strongest and most specific protein-peptide complex formations known, rivaling those of antibody-antigen interactions [De La Vega, Merino 2003;Gouaux and Mackinnon 2005;Ranganathan, Lewis 1996]. Kaliotoxin (KTX), a peptide neurotoxin from scorpion Androctonus mauretanicus venom, belongs to the charybdotoxin (CTX) family of $\mathrm{K}^{+}$channel-blocking scorpion toxins [Grissmer, Nguyen 1994;Lange, Becker 2005]. KTX inhibits the eukaryotic Kv1.3 channel both with high specificity and high affinity by binding with $1: 1$ stoichiometry to the outer pore region and blocking $\mathrm{K}^{+}$conduction [Miller 1995]. While several three-dimensional structures of unbound toxins and unbound $\mathrm{K}+$ channels were reported [Aiyar, Withka 1995;Doyle, Cabral 1998;Gouaux and Mackinnon 2005;Jiang, Lee 2002;Kuo, Gulbis 2003;Long, Campbell 2005;Miller 1995], a detailed view of the structural changes and motional degrees of freedom important for the formation of toxin-channel complexes is currently lacking. Recently, we have analyzed by solid-state nuclear magnetic resonance (ssNMR) spectroscopy the interaction of KTX with the chimaeric $\mathrm{K}^{+}$channel KcsA-Kv1.3 [Lange, Giller 2006]. Recognition of KTX by the channel was accompanied by conformational changes in both the KcsA-Kv1.3 outer pore region and in KTX. This observation was 
difficult to understand considering the principle, referred to as 'the induced fit' hypothesis [Koshland 1958], that conformational changes produce a high specificity in protein-ligand interactions at the expense of ligand affinity.

A more detailed insight into the molecular mechanism of KTX - KcsA-Kv1.3 and other ligand - membrane protein interactions requires the determination of amplitudes and time scales of motions related to complex formation. Such information is difficult to obtain from X-ray crystallography or mutant cycle analysis. NMR has provided much insight into the dynamical aspects of complex formation in solution [Kern and Zuiderweg 2003] but requires the use of ssNMR methods to study proteins in lipid bilayers. While good progress in the determination of 3D molecular structures from ssNMR data has been made [Castellani, Van Rossum 2002;Fossi, Castellani 2005;Lange, Becker 2005;Rienstra, Tucker-Kellogg 2002], no highresolution protein structure has been reported to date [Castellani, Van Rossum 2002;Fossi, Castellani 2005;Rienstra, Tucker-Kellogg 2002;Zech, Wand 2005].

To have a detailed understanding and to elucidate the importance of structural dynamics observed in KTX upon binding to the potassium channel, high-resolution structure of the kaliotoxin bound to the potassium channel is necessary. From the method developed in Chapter 3, in this chapter, we determine the high-resolution structure of KTX bound to a membrane-embedded potassium channel and an improved docking model for the KTX - KcsA-Kv1.3 complex is also proposed based on mutagenesis studies and chemical shift differences of KTX observed upon binding to the potassium channel. 


\subsection{Materials and Methods}

\subsubsection{Solid-state NMR data}

The strategy starts from a list of chemical shifts obtained from a series of twodimensional ${ }^{13} \mathrm{C},{ }^{13} \mathrm{C}$ - and ${ }^{15} \mathrm{~N},{ }^{13} \mathrm{C}$ - correlation experiments conducted on a uniformly $\left[{ }^{13} \mathrm{C},{ }^{15} \mathrm{~N}\right]$ labeled sample of KTX bound to KcsA-Kv1.3 under MAS conditions. Cross-peak lists of indirectly detected $\left({ }^{1} \mathrm{H},{ }^{1} \mathrm{H}\right)$ correlations obtained from $\mathrm{CHHC}$ [Lange, Becker 2005] spectrum with a $\left({ }^{1} \mathrm{H},{ }^{1} \mathrm{H}\right)$ mixing time of $325 \mu$ s was used as the input for the probabilistic assignment approach [Kuszewski, Schwieters 2004] described in Chapter 3. TALOS derived backbone dihedral angles [Cornilescu, Delaglio 1999] obtained from solid-state secondary chemical shifts $\left({ }^{13} \mathrm{C}_{\alpha},{ }^{13} \mathrm{C}_{\beta}\right.$ and ${ }^{15} \mathrm{~N}$ ) for KTX bound to potassium channel, also served as input for the structure calculation.

\subsubsection{Cross-peak assignment and Structure calculation}

The first step in this procedure is to assign the peak positions in the $2 \mathrm{D} \mathrm{CHHC}$ spectrum obtained at $325 \mu$ s mixing times. The probabilistic assignment algorithm for automated structure determination (PASD) [Kuszewski, Schwieters 2004] automatically performs this task by matching the assigned chemical shifts with the peak positions in 2D CHHC spectrum. Earlier, the backbone fold of KTX (Protein Databank (PDB) code: 1XSW) [Lange, Becker 2005] in the solid phase was deduced from 28 manually assigned interresidue CHHC correlations using a conventional simulated annealing protocol. Building on the backbone fold and the unassigned cross peaks lists, most of the peaks observed in 2D CHHC spectrum was here assigned using PASD. Subsequently, structure calculation was carried out in torsion angle space using the assigned distance restraints. Distance restraints were supplemented by 
torsion angle restraints derived from ${ }^{1} \mathrm{H},{ }^{15} \mathrm{~N},{ }^{13} \mathrm{C}$ chemical shifts using the program TALOS [Cornilescu, Delaglio 1999]. After completion of the PASD calculations, cross peak assignments were selected that had a likelihood of 1.0 (as defined by PASD). Selected assignments were then carefully verified by manual inspection of 2D CHHC and 2D NHHC spectra. In the final stage, manually verified cross peak assignments were used for calculation of a high-resolution structure using an optimized simulated annealing protocol [Linge, Williams 2003;Schwieters, Kuszewski 2003]. The calculations were started from random initial coordinates and all verified distance restraints were active during the course of calculation. In addition, torsion angle restraints derived from backbone chemical shifts were included.

\subsubsection{Modeling of KesA-Kv1.3}

Homology modeling of the chimaeric KcsA-Kv1.3 potassium channel was calculated using the crystal structure of conductive KcsA solved at $2 \AA$ resolution (PDB code 1K4C) [Zhou, Morais-Cabral 2001]. Positions R52, G53, A54, G56, A57, Q58, L59, I60, T61, Y62 and R64 in 1K4C were mutated to A52, D53, D54, T56, S57, G58, F59, S60, S61, I62 and D64, respectively in the model channel. Using KcsA as a template structure and the sequence alignment of KcsA with the model, generated with CLUSTALW [Thompson, Higgins 1994], 100 models were calculated using the program MODELLER [Marti-Renom, Stuart 2000]. The 20 best models were then energy minimized to remove steric overlaps and clashes of the side chains. The r.m.s. deviation between KcsA (1K4C) and the lowest-energy model was $0.334 \AA$. The average r.m.s deviation of the 20 lowest-energy models from the mean structure was 0.15 and $0.9 \AA$ for the backbone and heavy atoms, respectively. 


\subsubsection{Docking}

A model of the KTX-KcsA-Kv1.3 complex was calculated using the docking program HADDOCK version 1.3 [Dominguez, Boelens 2003]. The active and passive residues used for the docking in HADDOCK were chosen based on the chemical shift perturbation observed for KTX upon binding to the potassium $(\mathrm{K}+)$ channel and relative residue solvent accessibility as calculated by the program NACCESS [Hubbard and Thornton 1993]. Active residues were those residues, for which the chemical shift differences were larger than the mean value (1.675 ppm for KTX 0.509 ppm for KcsA-Kv1.3) and the relative residue solvent accessibility (as calculated by NACCESS) exceeded $50 \%$ for either side chain or backbone atoms. Passive residues were defined as the neighbors of the active residues whose relative residue solvent accessibility was larger than 50\%. Thus, five amino acids (M23, R24, K27, M29 and T36) of KTX and three amino acids (D64, E71 and D80) from each subunit of the tetrameric KcsA-Kv1.3 were defined as active residues. Besides, we defined five passive amino acids (F25, G26, C28, R31, K32) in KTX and three (I62, P63, L81) in each subunit of the tetrameric structure of KcsA-Kv1.3. In HADDOCK, the docking is driven by ambiguous intermolecular restraints (AIRs) [Nilges 1993]. An AIR is defined as an ambiguous intermolecular distance with a maximum value of $3.0 \AA$ between any atom of an active residue of KTX and any atom of both active and passive residues of KcsA-Kv1.3 and inversely for KcsA-Kv1.3 with the toxin KTX. The ensemble of 20 structures of the bound KTX, i.e. the 3D structure of KTX determined in this study using solid-state NMR spectra recorded on uniformly labeled [13C, 15N] KTX in complex with KcsA-Kv1.3 reconstituted into proteoliposomes, was used as a starting conformer for the toxin and kept rigid throughout the docking protocol. For KcsA-kv1.3, the lowest-energy model obtained by homology modeling 
was used. Restraints for backbone torsion angles obtained for residues within the selectivity filter of KcsA-Kv1.3 from solid-state secondary chemical shifts [Lange, Giller 2006] by the program TALOS [Cornilescu, Delaglio 1999] were used and the respective residues (T75 to G79) were kept rigid during the docking. All other active and passive residues were defined as semi-flexible segments during the docking. 


\subsection{Results and Discussions}

\subsubsection{Solid-state NMR structure of KTX in complex with KesA-Kv1.3}

The approach was used to solve the high-resolution 3D structure of the 38-residue potassium channel blocker toxin KTX existing in complex with membrane-embedded KcsA-Kv1.3. ${ }^{1} \mathrm{H}-{ }^{1} \mathrm{H}$ correlations were detected in $2 \mathrm{D}$ CHHC spectrum with mixing times of $325 \mu \mathrm{s}$. Proton-proton correlation 2D CHHC spectrum of the solid-state was automatically peak picked using SPARKY 3 (T. D. Goddard and D. G. Kneller, SPARKY 3, University of California, San Francisco). Diagonal peaks were manually removed. Peak intensities obtained from the 2D CHHC spectrum was classified into four ranges and converted into distance ranges of 1.8-2.7, 1.8-3.3, 1.85.0 and 1.8-6.0 А, respectively. For analysis of the solid-state spectra by PASD, ${ }^{13} \mathrm{C}$ and ${ }^{15} \mathrm{~N}$ chemical shifts were labeled as if they were proton chemical shifts. The tolerances for matching chemical shifts to cross-peaks were set to $0.38 \mathrm{ppm}$ in the acquisition dimension and to $0.60 \mathrm{ppm}$ in the indirect dimension. PASD structures do not represent fully-refined NMR structures [Kuszewski, Schwieters 2004]. Therefore, we selected cross-peaks with a final likelihood assignment (as defined by PASD) of 1.0. For KTX bound to the potassium channel, $19 \%$ of all long-range restraints, $76 \%$ of the medium-range restraints, and $100 \%$ of the sequential restraints had final restraint likelihoods of 1.0. All other restraints had final restraint likelihoods of 0 . Assignments obtained for these cross-peaks by PASD were verified by manual inspection of the 2D CHHC spectrum.

A large number of distance restraints were unambiguously assigned in solidstate NMR spectra recorded on proteoliposomes containing uniformly labeled $\left[{ }^{13} \mathrm{C}\right.$, ${ }^{15} \mathrm{~N}$ ] KTX bound to purified KcsA-Kv1.3 (Table 4.1). The structures based on distance and dihedral angle restraints shows very well defined secondary structure 

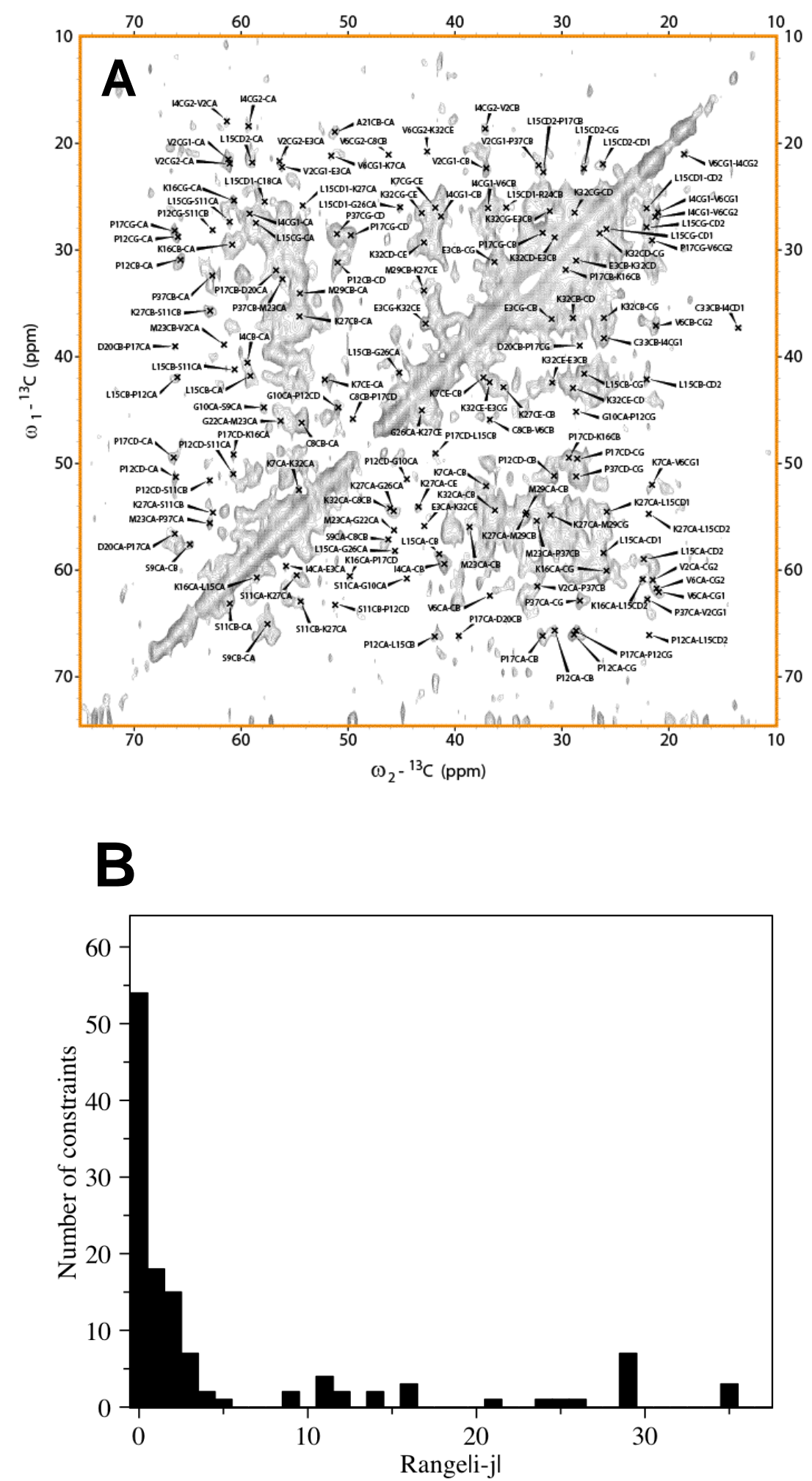

Figure 4.1: Interresidue correlations observed in $\mathrm{U}-\left[{ }^{13} \mathrm{C},{ }^{15} \mathrm{~N}\right]-\mathrm{KTX}$ in complex with unlabeled KcsA-Kv1.3. (A) 2D CHHC spectrum recorded with a mixing time of $325 \mu \mathrm{s}$. Unambiguously assigned signals are labeled. (B) Number of unambiguously assigned $\mathrm{CHHC}$ distance constraints as a function of residue difference $i$ and $j$. 
Table 4.1: Structural statistics for the 20 lowest-energy structures of KTX in com plex with KcsA-Kv1.3.

\section{Proton-Proton distance correlations}

$\begin{array}{lc}\text { Total } & 113 \\ \text { Short range } & 70 \\ \text { Medium range } & 18 \\ \text { Long range } & 25\end{array}$

Distance violations $(>0.5 \AA) \quad 1$

\section{Dihedral angles}

Total

Angle violations

54

$\operatorname{Energy}(\mathbf{k c a l} / \mathbf{m o l})$

Total

3

Bond

Angle

Improper

165.25

Dihedral

46.78

NOE

128.31

Coordinate precision ${ }^{\mathrm{a}}$

Backbone RMSD $(\AA) \quad 0.5$

Heavy side chain RMSD $(\AA)$

$\underline{\text { Ramachandran statistics }}$

Most favored region (\%) $\quad 83.0$

Additionally allowed region (\%) $\quad 3.7$

Generously allowed region (\%) $\quad 3.3$

Disallowed region $(\%) \quad 0.0$

${ }^{a}$ Defined as the average rmsd difference between the 20 structures and the mean coordinates. 

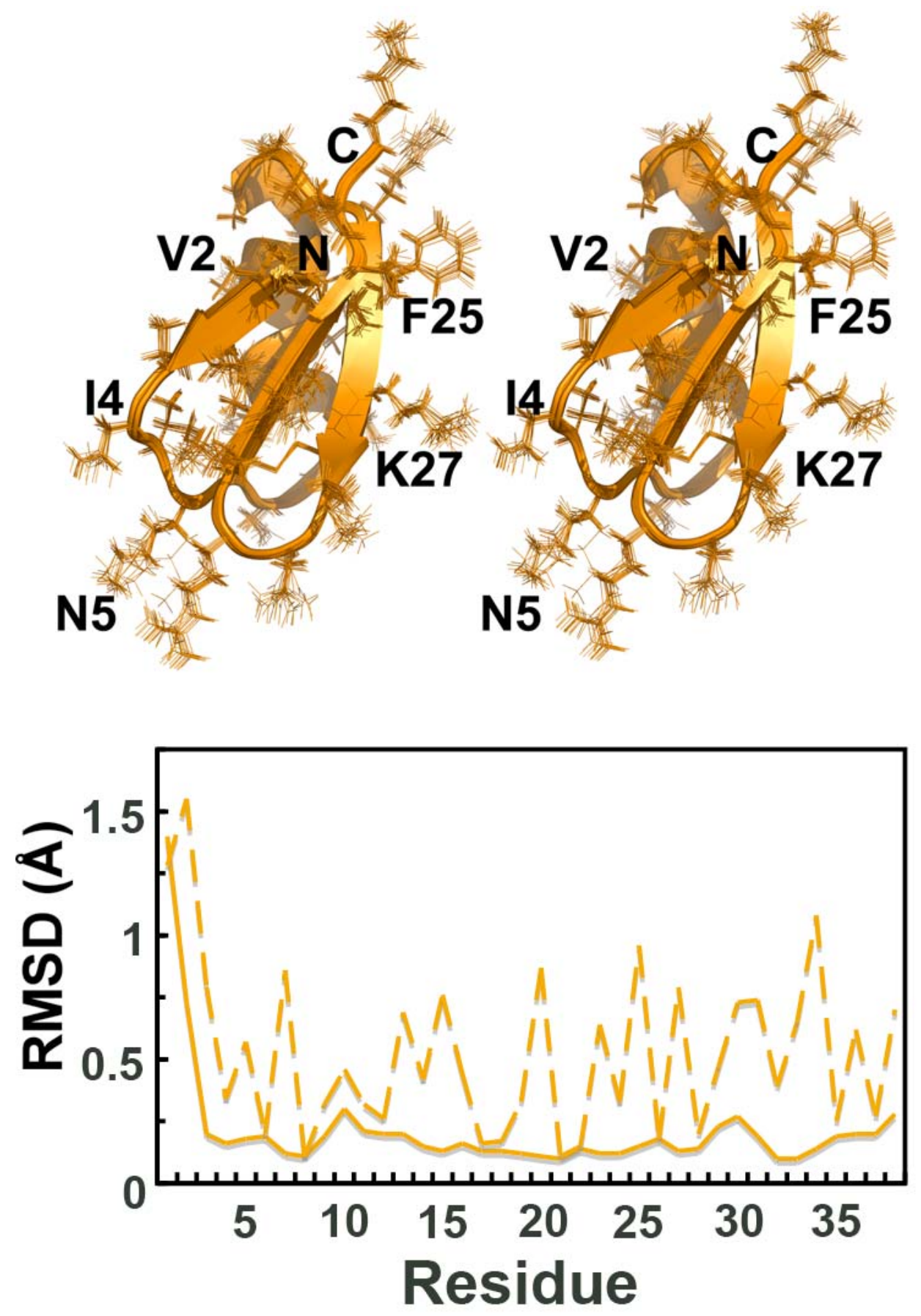

Figure 4.2: Stereo view of 20 lowest energy ensemble of high-resolution solidstructure of Kaliotoxin in complex with KcsA-Kv1.3 (upper). Residue based rms deviation of the atomic coordinates within the ensemble of 20 lowest energy structures (lower). Deviations for the backbone atoms and for the non-hydrogen side chain are shown as solid and dashed line, respectively. 
elements consisting of $\alpha$-helix affixed by disulphide bridges to a three-stranded $\beta$ sheet, that is typical for the charybdotoxin family of $\mathrm{K}+$ channel-blocking scorpion toxins [Grissmer, Nguyen 1994], with rms deviations in the backbone and heavy atoms of 0.5 and $1.2 \AA$, respectively, for all residues. The backbone and side chains in the resulting structures of KTX in complex with the $\mathrm{K}^{+}$channel were well defined as seen from Figure 4.2 and Table 4.1. $83 \%$ of residues were found in the most favored region of Ramachandran plot and none of the 38-residues was found in the disallowed region.

At the end of pass 1 and 2 the PASD algorithm calculates likelihood estimates that each particular assignment associated with a cross-peak is correct. The likelihoods are calculated using the ensemble of structures present at the end of the corresponding pass. Thus, they are a metric of how consistent a given assignment is with the ensemble of structures at the end of each calculation pass. Here we have not used the ensemble of structures present at the end of pass 1 and 2 for calculation of likelihood estimates, but the high-resolution structures of KTX obtained under different conditions (solution-state or solid-state free KTX). This improved convergence in the structure calculations and was justified as we previously established that the fold of KTX in solution and in the solid phase is the same. For each state of KTX, in complex with the KcsA-Kv1.3, the structure calculation was done twice, in which the high-resolution structures of the two other states were used for calculation of the likelihood estimates. The PASD calculations of KTX(solid/bound) were done once by using KTX(solution) for calculation of the likelihood estimates at the end of pass 1 and 2. Then a second PASD calculation was done, in which the likelihood estimates were determined using KTX(solid/free). Thus, the PASD calculations were biased on purpose toward the structure of the other state. 
In both cases, the structures obtained for KTX in complex with the channel were indistinguishable. Note, that identical structure calculation protocols were used in all cases.
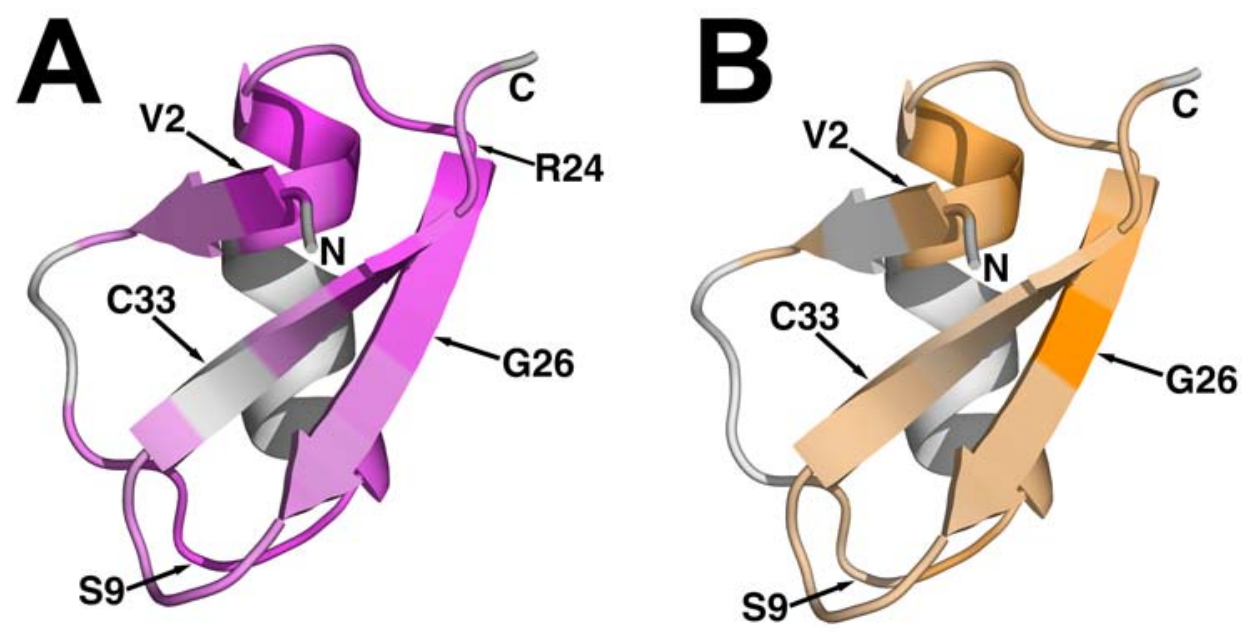

Figure 4.3: (A) Structural differences between KTX in solution and the KTX in complex with the potassium channel in solid phase mapped onto the solution-state structure (pink scale). (B) Structural differences between between free KTX in the solid phase and in complex with the potassium channel mapped onto the solutionstate structure (orange scale).

\subsubsection{Comparison with high-resolution solution-state and solid-state} (free) NMR structures of KTX

Differences in the backbone conformation between KTX in solution and KTX in complex with the potassium channel in the solid phase is shown in Figure 4.3A. Residue-based root mean square deviations (one-residue-window) were mapped onto the solution-state structure using a continuous pink scale. Also in Figure 4.3B, differences in the backbone conformation between free KTX in the solid phase and in complex with the potassium channel is shown. Structural differences are mapped onto the solution-state structure using a continuous orange scale. Mapping the structural differences onto the structure of KTX indicated that they mainly occurred in one particular half of the KTX molecule including the C-terminal end of the $\alpha$-helix, the 
$\mathrm{N}$-terminal half of the second $\beta$-strand and the Cys18-Cys35 disulfide bridge connecting $\alpha$-helix and third $\beta$-strand. The differences between solution and solidstate structures of KTX correlate well with those that were observed upon binding of KTX to the KcsA-Kv1.3 channel. This correlation indicates that transitions between different conformational states of KTX involve flexibility at specific points in the $\beta$ sheet of the KTX polypeptide.

\subsubsection{Improved model of KTX-KesA-Kv1.3}

We used the high-resolution 3D structure of bound KTX, the backbone conformation of the selectivity filter of KcsA-Kv1.3 in the complex and functional data to derive an improved model for the KTX - KcsA-Kv1.3 complex (Figure 4.4). Docking of KTX with the model of the KcsA-kv1.3 was performed with HADDOCK version 1.3 in combination with CNS version 1.1 [Brunger, Adams 1998;Dominguez, Boelens 2003]. The starting structures for the docking were the 20 solid-state NMR structures of the bound KTX and the model of the KcsA-Kv1.3 obtained above. In total, 2000 rigid-body docking solutions for the ensemble of 20 NMR structures of bound KTX were first generated by energy minimization. In the initial stages, and the docking is mainly based on the AIRs. Once the structures are within the non-bonded cut-off of 8.5 , van der Waals and electrostatic energy terms become important. The 200 best solutions, based on the intermolecular energies, were subjected to semi-flexible simulated annealing in torsion angle space followed by a final refinement in explicit water. During simulated annealing and water refinement, residues at the interface, both side chains and backbone, were allowed to move to avoid steric overlaps and clashes. 

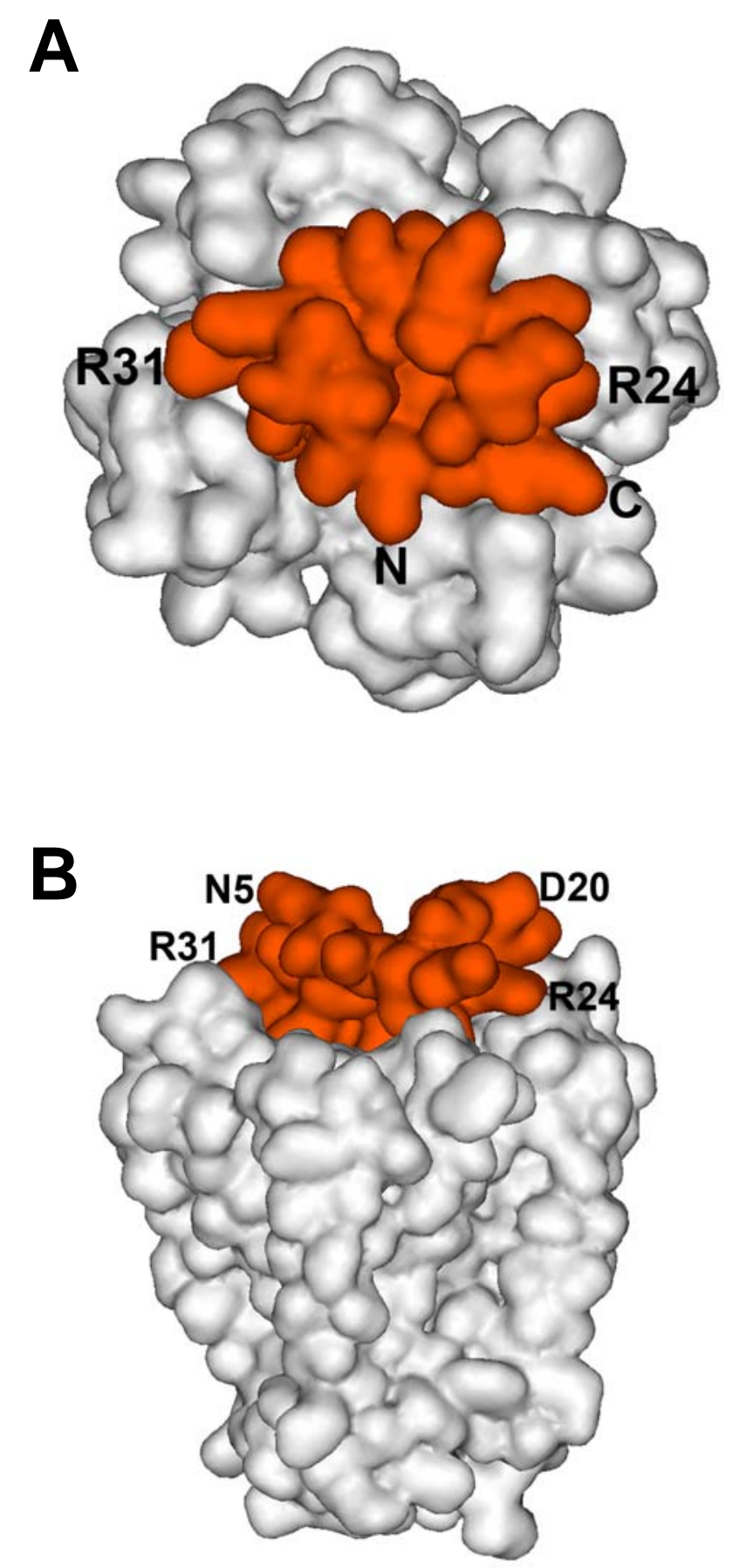

Figure 4.4: Model of the KTX - KcsA-Kv1.3 complex shown in different views. In (B), residues 149-160 of KcsA-Kv1.3 were removed to allow a better view of bound KTX. 


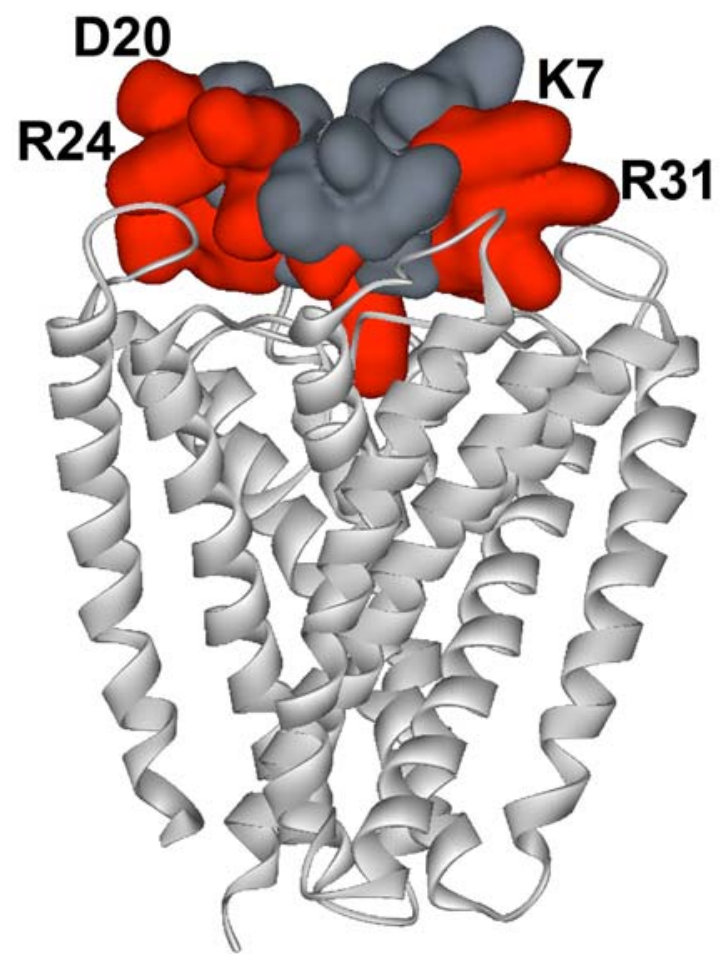

Figure 4.5: Model of the complex between KTX and KcsA-Kv1.3 (Protein Data Bank 1K4C. KTX residues that are important for binding to the channel according to mutagenesis studies are highlighted in orange.

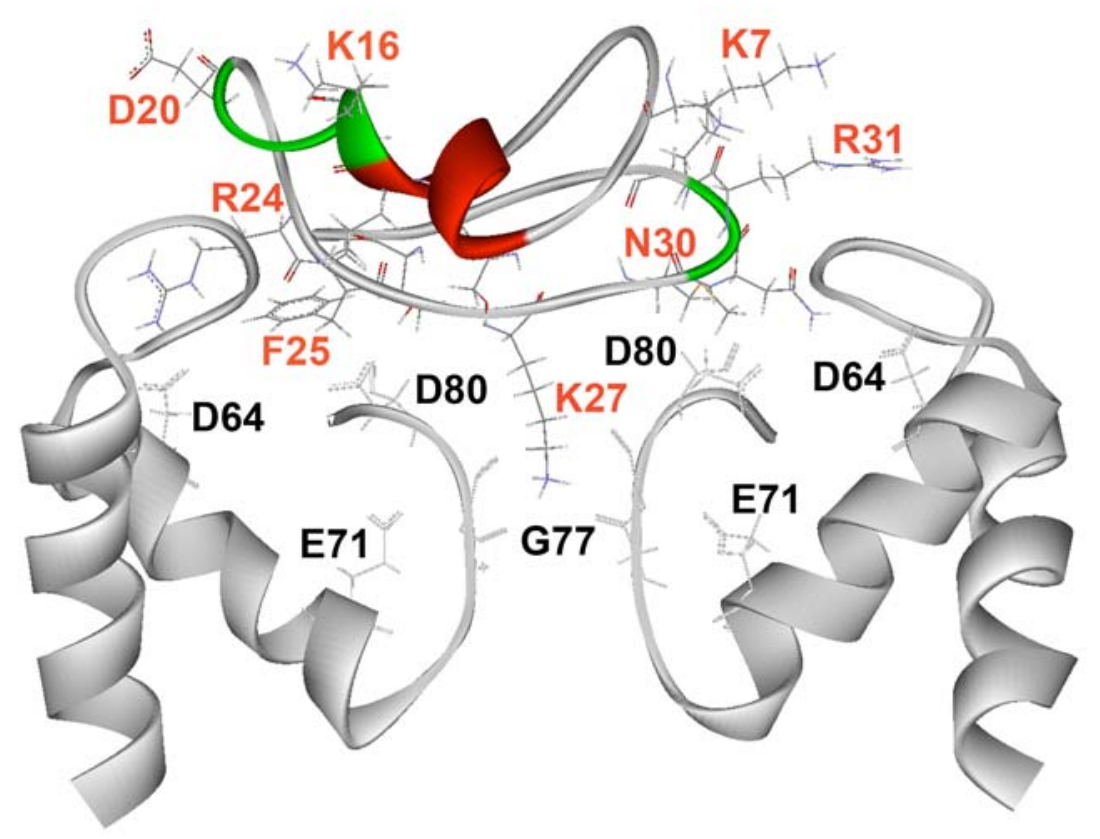

Figure 4.6: Lys 27 of KTX deeply inserts into the pore region the KcsA-Kv1.3 


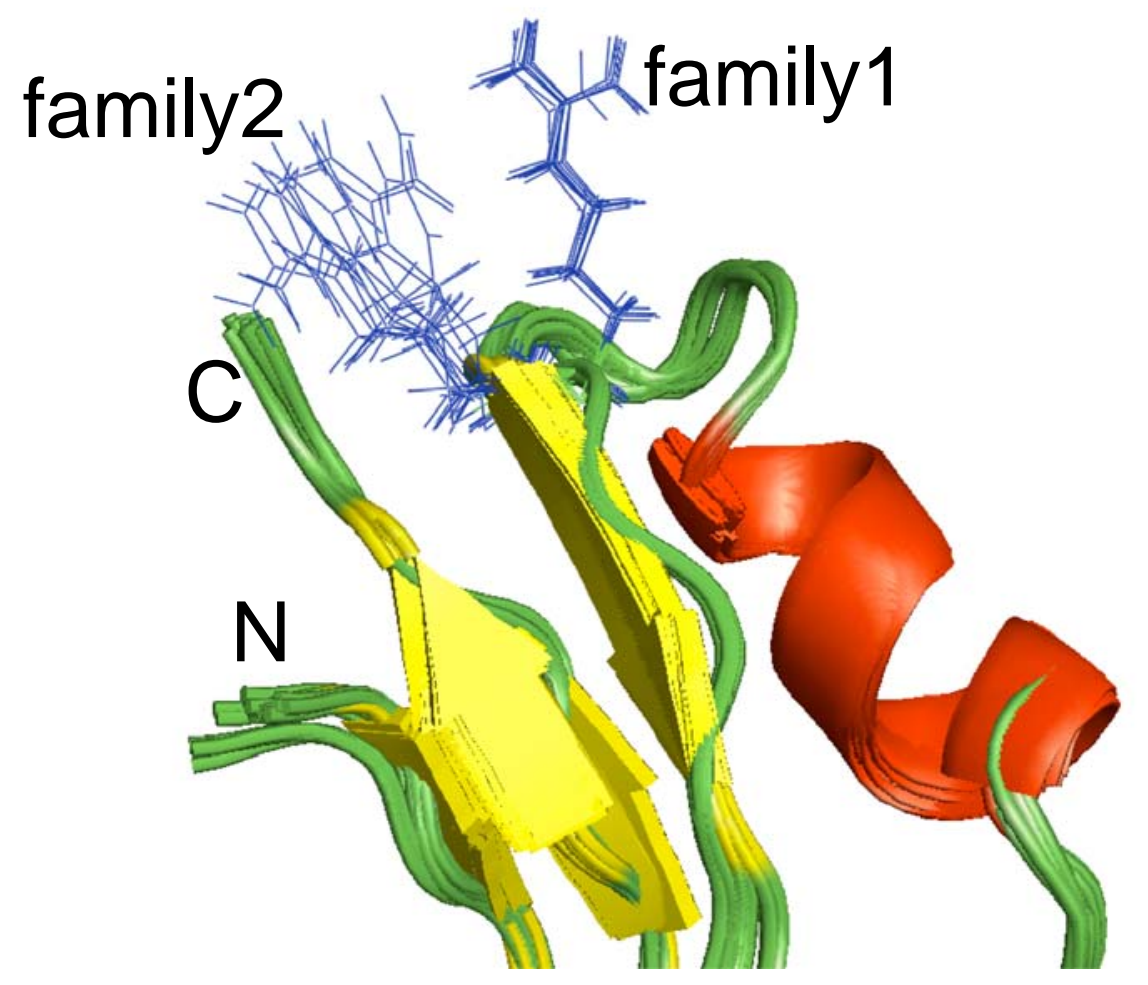

Figure 4.7: Cartoon representation of two families of structures. Two families differed in the orientation of the side chain of R24.

The final 200 structures were clustered into two families based on pairwise backbone r.m.s.d. 20 lowest energy structures were selected in each family. The average r.m.s.d values to the mean structure were 0.45 and $0.46 \AA$ for the backbone atoms of cluster 1 and cluster 2, respectively and the average r.m.s.d to the mean structure were 0.75 and $0.73 \AA$ for the side chain atoms of cluster1 and cluster 2 , respectively. The Lowestenergy complex from cluster 1 is shown in the Figure 4.5. Structures in cluster 2 differed from those in cluster 1 by a $180^{\circ}$ rotation relative to the structure of KcsAKv1.3. We used the high-resolution 3D structure of bound KTX, the backbone conformation of the selectivity filter of KcsA-Kv1.3 in the complex and functional data to derive an improved model for the KTX - KcsA-Kv1.3 complex (Figure 4.5). The positively charged side chain of Lys27, which is highly conserved in scorpion toxins, protrudes from the interaction surface and plugs the $\mathrm{K}+$ channel pore and comfortably fits into the selectivity filter near G77 (Figure 4.6). 
Structure calculation for KTX(solid/bound) resulted in two families that had very similar backbone conformations (maximum r.m.s. deviation of $0.3 \AA$ ), but differed in the orientation of the side chain of R24 (Figure 4.7). Out of 50 calculated structures, 30 belonged to family 1 and 20 to family $2 .{ }^{13} \mathrm{C}_{\alpha},{ }^{13} \mathrm{C}_{\beta}$ and ${ }^{15} \mathrm{~N}$ chemical shifts back-calculated from each family using SHIFTX did fit to a comparable degree to experimental values. However, the ${ }^{13} \mathrm{C}_{\alpha}$, chemical shift predicted for R24 from family 2 structures differed by $0.9 \mathrm{ppm}$ from the experimental value, whereas the value predicted for family 1 conformations differed by only $0.1 \mathrm{ppm}$. In addition, the ${ }^{15} \mathrm{~N}$ chemical shift predicted for M23 on the basis of family 1 was much closer to the experimental value $(0.6 \mathrm{ppm}$ difference instead of $5.4 \mathrm{ppm}$ difference in case of family 2). When compared to the solid-state structure of free KTX, a smaller structural change is present in family 1 conformations. Both the family 1 and family 2 ensembles were used for docking of KTX to KcsA-Kv1.3. Only in docking solutions obtained with family 1 structures of $\mathrm{KTX}$ (solid/bound), R24 was able to form a hydrogen bond with D64. The R24-D64 hydrogen bond is thought to be important for binding of KTX to $\mathrm{K}+$ channels according to mutagenesis studies. These data suggest that the orientation of the side chain of R24 seen in the family 1 ensemble is the conformation R24 adopts in complex with KcsA-Kv1.3. For further analysis, therefore, only family 1 of KTX(solid/bound) was used. 


\subsection{Conclusions}

In this study, the previously developed approach (Chapter 3) allowed the determination of high-resolution structure of KTX bound to a membrane-embedded potassium channel, KcsA-Kv1.3. An improved model of KTX-KcsA-Kv1.3 was presented based on the functional data and chemical shift differences observed for KTX upon binding to the tetrameric KcsA-Kv1.3. In consistent with the previous results and observed data, Lys 27 protrudes from the interaction surface of the KTX and inserts deeply into the selectivity filter of the chimaeric channel. Though, the structure of the bound KTX could be improved by measuring spectra at different mixing times, as it was done for KTX in free form, the overall quality of the structure and the data presented would still hold true. High-resolution structural data along with dynamics studies at the interfacial regions would provide the basis for the formation of a tight complex with the active site of the $\mathrm{K}+$ channel. 


\section{Chapter 5}

\section{Understanding and Prediction of alignment and residual dipolar couplings of a protein from its known three- dimensional structure}

\subsection{Introduction}

\subsubsection{Residual Dipolar Couplings (RDCs)}

Residual dipolar coupling (RDCs) (§ 1.2.4.3 Residual Dipolar Couplings) offers new opportunities in NMR studies of macromolecules existing in solution [Bax 2003;Prestegard, Al-Hashimi 2000]. This anisotropic interaction contains valuable structural information; hence it is used to address a number of different problems in structural biology. These range from structure validation, to high-resolution structural applications to proteins, to the determination of inter domain orientation in multi domain or multi subunit systems, to characterization of structure and dynamics of proteins and non-protein systems [Clore and Garrett 1999;Cornilescu, Marquardt 1998;Delaglio, Kontaxis 2000;Fischer, Losonczi 1999;Hus, Marion 2001;Jung, Sharma 2004;Markus, Gerstner 1999;Meiler, Prompers 2001;Skrynnikov, Goto 2000; Tolman 2002]. This anisotropic interaction is not normally observable in highresolution NMR because, it effectively average to zero with molecular tumbling [Prestegard 1998]. However, they can be observed, if small degrees of molecular order are induced. The desired very weak ordering (on the order of $10^{-3}$ ) or in other words the partial alignment of the biomolecule in solution is usually accomplished by means of a dilute liquid crystalline medium (Figure 5.1) or an anisotropically compressed hydrogels [Barrientos, Dolan 2000;Barrientos, Louis 2001;Clore, Starich 
1998;Fleming, Gray 2000;Hansen, Mueller 1998;Prestegard and Kishore 2001;Ruckert and Otting 2000;Tjandra and Bax 1997]. The resulting residual dipolar interactions are scaled down by three orders of magnitude relative to their static values, but nevertheless and because of that can be measured at high accuracy.

It is important to introduce a controllable, but small amount of alignment. Under these conditions, the rotational diffusion of the protein is more or less unhindered, thus maintaining the narrow linewidth typical of liquid state NMR. The partial alignment results also in a net alignment, which allows the measurement of RDCs only for those nuclei that are very strongly coupled. So, the small tunable degree of alignment provides the structural information and narrow lines. RDCs can also be measured in the absence of any liquid crystalline medium when the molecule of interest has a sufficiently large intrinsic anisotropy of its magnetic susceptibility to achieve a preferred orientation with respect to the external magnetic field [Tolman, Flanagan 1995]. This has been shown for both paramagnetic and diamagnetic biomolecules [Contreras, Ubach 1999]. Even for paramagnetic proteins, the natural alignment with a high magnetic field is usually one order of magnitude smaller than that achieved by the use of an orienting medium.

It has been found that not only the degree of alignment, but also its characteristics can be modified by changing the medium. This property turns out to be useful because, if the structure of the macromolecule is assumed to be independent of the medium, the availability of RDCs for different alignment provides a set of independent data [Al-Hashimi, Valafar 2000;Peti, Meiler 2002]. A clear understanding of the dependence of the orientational behavior of a macromolecule on the structure of the aligning medium has not been reached, yet. Alignment would be the result of steric repulsions, which are likely to be similar in the various systems, and electrostatic interactions, which on the contrary, are expected to depend on the 
features of the colloidal suspensions. It was previously demonstrated that in a bicelle medium, consisting of large diameter, disk shaped zwitterionic phospholipid micelles; the principal axes of the molecular alignment tensor closely coincide with those of the rotational diffusion tensor [De Alba, Baber 1999;Tjandra and Bax 1997;Tjandra, Omichinski 1997]. This shows that in this nearly neutral medium, alignment is solely defined by the solute's shape. There also have been attempts to provide theoretical predictions of macromolecular alignment. Previously, in a computational approach implemented in

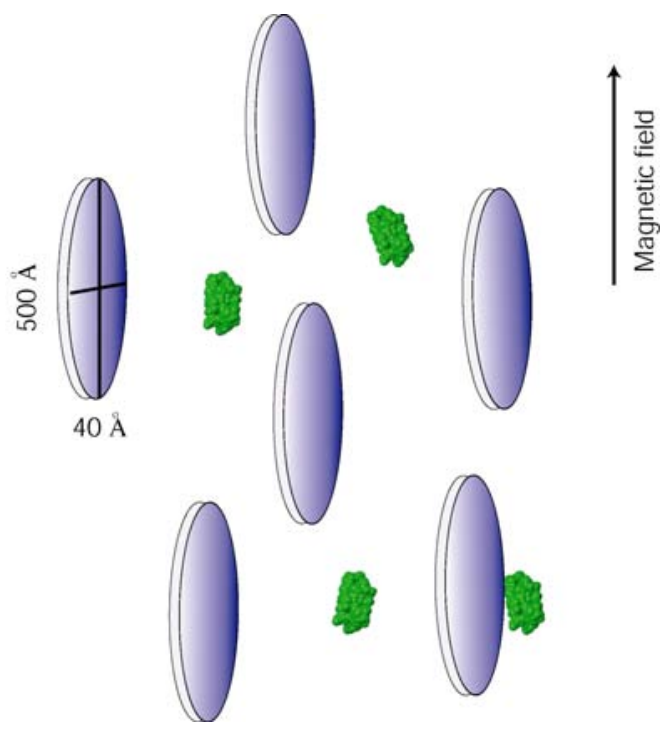

Figure 5.1: Alignment of biomolecules in dilute liquid crystalline media.

the program called Prediction of ALignment from Structure (PALES) [Zweckstetter and Bax 2000], it was shown that the magnitude and orientation of the steric component of the molecular alignment could be predicted accurately from the solute's three-dimensional shape. This was done, under the assumption that there are no attractive or long-range repulsive interactions between the solute and the disk or rod shaped liquid crystal particles; steric obstruction effects dominate solute alignment. In this model, the alignment tensor is calculated by averaging over all non-overlapping configurations of a liquid crystal particle and macromolecule. The macromolecular 
shape is represented with atomistic detail, whereas bicelle was modeled as an infinite wall. This method's ability to predict a protein alignment tensor and hence the dipolar coupling allowed an opportunity in validating structural models [Bewley 2001;Clore and Garrett 1999], determining relative orientation of protein domains [Bewley and Clore 2000], refining nucleic acid structure [Warren and Moore 2001], differentiating monomeric from homodimeric states [Zweckstetter and Bax 2000]. The ability to predict dipolar couplings for a given protein structure also provides unique opportunities when attempting to classify protein fold families on the basis of unassigned NMR data, potentially increasing data throughput in structural genomics [Valafar and Prestegard 2003].

However, when a net charge was added to the bicelles [Ramirez and Bax 1998], the alignment tensor of the protein showed a modification, demonstrating that electrostatic interactions could play a role. As a further support to this view, it has been observed that alignment in virus suspensions can be tuned by changing the ionic strength: the differences with respect to bicellar suspensions are gradually reduced when the ionic strength is increased [Zweckstetter and Bax 2001]. As a direct consequence, the prediction of the macromolecular alignment tensor was less accurate based on the steric obstruction alone for negatively charged liquid-crystalline media. Analysis of solute alignment observed in apolar organic liquid-crystalline media shows that steric interactions frequently dominate, but electrostatic forces could be important [Dingemans, Photinos 2003;Terzis, Poon 1996]. There is still debate as to which electrostatic interactions are most important. Some studies argue that the dipole moment plays a major role [Photinos, Poon 1992;Photinos and Samulski 1993]. Other studies indicate that in apolar media it is primarily quadrupole moment of the solute that dominates its alignment [Syvitski and Burnell 1997;Syvitski and Burnell 2000]. Full calculation of electrostatic forces between charged macromolecules in aqueous 
solution is very complex and computationally demanding [Davis and Mccammon 1990;Honig, Sharp 1993].

Recently, a simple extension of the steric obstruction model, which combined the short-range steric and long-range electrostatic interactions, allowed improved predictions of the magnitude and orientation of the alignment tensor of protein and DNA solutes in charged nematic media, such as widely used medium of filamentous phage Pf1 [Zweckstetter, Hummer 2004] (Figure 5.2). Filamentous phage was modeled as an infinitely long cylinder with uniform charge distribution on its surface. The extended model approximates the electrostatic interaction between a solute and the alignment media as that between the solute's surface charges and electric field

\section{Steric obstruction model}

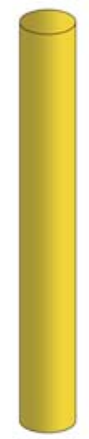

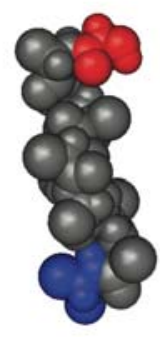

allowed

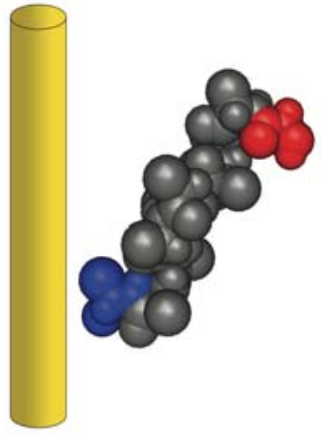

allowed

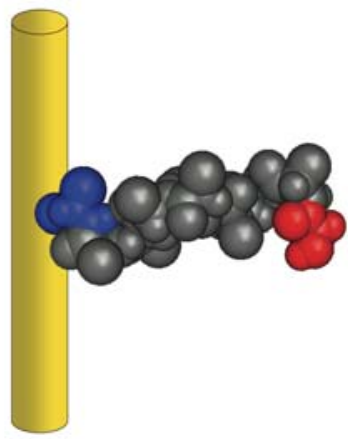

forbidden

\section{Electrostatic model}
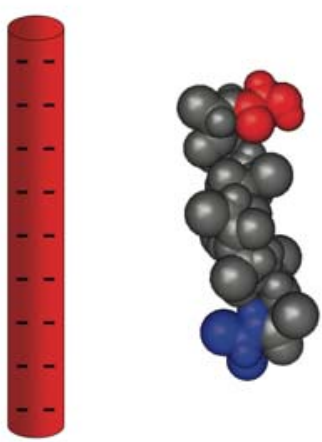

allowed
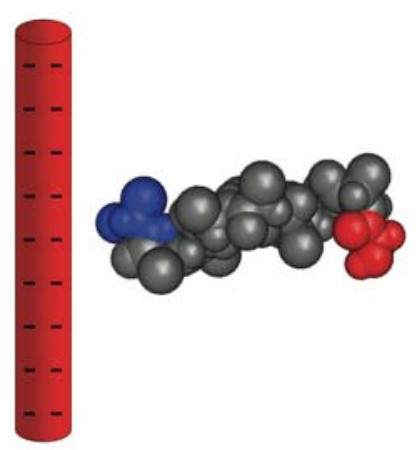

preferred

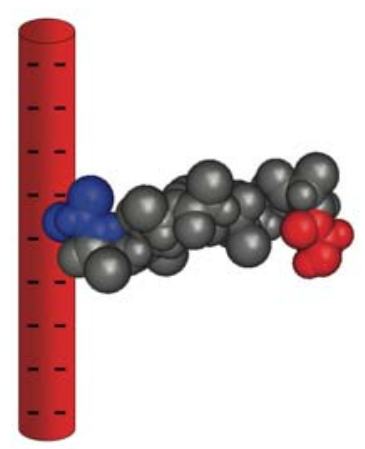

forbidden

Figure 5.2: Schematic representation of the steric and electrostatic PALES [Zweckstetter and Bax 2000;Zweckstetter, Hummer 2004]. For the allowed and preferred orientation of the biomolecules, individual alignment tensors were weighed by the relative electrostatic contribution through Boltzmann probability. 
strength generated by the medium. Hence the model is not limited to neutral media such as bicells and alkyl poly-(ethylene glycol)-based liquid crystals, but extends to charged liquid-crystalline media. Recently, a similar approach to predict alignment in charged nematic media [Ferrarini 2003], was also reported.

Though, the overall quality was good considering the simplified electrostatic model, there were few notable problems. Due to the continuum level treatment of molecular solvents, which excludes the tight ionic interactions at low salt concentration and ion packing contributions which plays a role at high salt concentrations, the magnitude of the predicted alignment tensors varied considerably from the observed values, for salt concentrations between $0.1 \mathrm{M}$ and $0.3 \mathrm{M}$ and the predictions varied for different proteins. Also the uniform charge distribution on the liquid crystal particle rules out the possibility of the specific interactions with the interfacial charge distribution, when the protein is close to the interface. Rigid approximation of the protein structure and charge distribution on the protein and interface may lead to errors for proteins with flexible charged side chains that could adjust their conformations to optimize electrostatic interactions with interface. Because minor rearrangement of side chains of charged surface residue dramatically affected the prediction. This may be either due to the quality of the structural model or due to less detailed charged model. Hence demanding for a detailed atomistic treatment of the solute and solvents.

Here in this study we focus on the incorporation of improved electrostatic model in PALES to predict the molecular alignment and residual dipolar couplings of a protein from its known three-dimensional structure. Detailed electrostatic calculations were performed, by solving nonlinear Poisson-Boltzmann equations for the protein/bilayer system, where protein and bilayer were represented in atomic details. Nonlinear Poisson-Boltzmann equation was solved for different orientations and 
translational positions of a protein in front of a liquid crystal particle. Electrostatic potentials and free energies were then used within PALES to calculate the alignment tensor and the residual dipolar couplings of the protein. Dipolar couplings calculated from these electrostatic free energies will be compared to experimental values, which had been measured in the Rec A binding protein DinI dissolved in Pf1 bacteriophage. Purple membranes, like bacteriophage Pf1, are highly negatively charged, and the alignments in both cases are dominated by electrostatic interactions. It was shown previously that, observed alignment tensors for proteins dissolved in purple membrane or bacteriophage Pf1 are similar [Koenig, Hu 1999;Lakomek, Carlomagno 2006;Lakomek, Griesinger (personal communications)], depending on salt concentrations of the solute medium. These findings provide experimental evidence supporting our case of using RDCs measured in bacteriophage Pf1 medium for the calculation of alignment tensors in membrane system (here lipid bilayers). Although short-range interactions between the liquid crystal particle and the protein are not taken correctly into account in this manner, the influence of the protein on the overall electrostatic potential can be taken into account explicitly. Thus, we can overcome one of the major limitations of the simple electrostatic model currently used in PALES. Solving a more detailed electrostatic model expected to improve the quality of the charge/shape prediction of the alignment.

\subsubsection{Electrostatics}

Electrostatic interactions play an important role in both the structure and function of molecules [Honig, Sharp 1993]. The complex shape of the macromolecules dramatically affects their electrostatic field, and can be crucial to their function. The finite difference Poisson-Boltzmann (FDPB) method [Davis and Mccammon 
1990;Honig and Nicholls 1995;K. A. Sharp and B. Honig 1990] has been widely applied to describe the electrostatic properties of proteins, nucleic acids, and membranes [Baker and Mccammon 2003]. In a series of experimental/theoretical studies over the past decade, several research groups have used this method to describe the binding of charged peptides and proteins to membrane surfaces. These works demonstrated that the FDPB method is remarkably accurate in treating electrostatic properties associated with non-specific binding. This is because many of the relevant interactions are long range and depend only on the charge distribution of the system. The computational methodology is based on finite difference solutions to the full (nonlinear) Poisson-Boltzmann (PB) equation, an equation of classical electrostatics [Gallagher and Sharp 1998;Honig and Nicholls 1995;Holst 1993;Gilson 1988;Kim A. Sharp and Barry Honig 1990;K. A. Sharp and B. Honig 1990]. The finite difference method is a numerical procedure that allows the solution of the PB equation for biological molecules that often have complex shapes. The PB equation is as follows:

$$
\nabla[\varepsilon(r) \nabla \varphi(r)]-\varepsilon_{r} \kappa(r)^{2} \sinh [\varphi(r)]+e^{2} /\left(\varepsilon_{0} k_{B} T\right) \rho^{f}(r)=0
$$

where $\varepsilon(r)$ is the dielectric constant, $\varphi(r)$ is the electrostatic potential, $\kappa(r)$ is the Debye-Hückel parameter and $\rho^{f}(r)$ is the fixed charge density of the protein and lipids; all four variables are functions of space, $r=(x, y, z)$. A protein/membrane system is represented atomistically and is mapped onto a three-dimensional lattice of points; each lattice point represents a small region of the protein or membrane $(\varepsilon=2$; to approximate electronic polarizability) or the aqueous phase $(\varepsilon=80$; to account for the highly polar nature of water molecules).

The solutions to the PB equation are the electrostatic potentials, $\varphi(r)$, which are 
used to calculate the electrostatic free energy of a system, $G_{e l}[$ Kim A. Sharp and Barry Honig 1990]. The electrostatic free energy of interaction between protein and membrane, $\Delta \mathrm{G}_{\mathrm{el}}$, is calculated as the difference between the electrostatic free energy of the protein and membrane when they are close together, $G_{\mathrm{el}}(\mathrm{P} . \mathrm{M})$, and when they are far apart, $\mathrm{G}_{\mathrm{el}}(\mathrm{P})$ and $\mathrm{G}_{\mathrm{el}}(\mathrm{M})$ [Ben-Tal, Honig 1996]:

$$
\Delta G_{e l}=G_{e l}(P . M)-\left[G_{e l}(P)+G_{e l}(M)\right]
$$

When the protein is far from the membrane surface, it experiences an electrostatic attraction that drives it toward the negatively charged membrane. This "Coulombic" attraction increases as the protein approaches the membrane. Close to the membrane surface, charged and polar groups on the both the protein and membrane are desolvated, or stripped of water molecules, which is energetically unfavorable and results in a repulsion at small distances. The minimum electrostatic free energy of interaction of protein is predicted to occur where the van der Waals surface of the peptide and membrane are separated by a distance of about the thickness of a layer of water. At this distance the Coulombic attractive force is balanced by the repulsive desolvation force. The components of the electrostatic free energy of interaction, namely the Coulombic attraction and the desolvation repulsion, can be calculated explicitly. The desolvation penalty $\Delta \mathrm{G}_{\text {desolv }}$ is determined at each $\mathrm{R}$ by calculating to what extent the presence of the protein and the membrane shield the others from favorable interactions with solvent [Misra and Honig 1995;Misra, Sharp 1994]. This is done by discharging the protein and membrane in turn and calculating the electrostatic free energy of the system:

$$
\Delta G_{\text {desolv }}=\Delta G_{e l}(P(Q=0) \cdot M)+\Delta G_{e l}(P \cdot M(Q=0))
$$

The Coulombic component $\Delta \mathrm{G}_{\text {Coul }}$ is simply the difference between the total electrostatic free energy of interaction and the desolvation penalty: 


$$
\Delta G_{\text {Coul }}=\Delta G_{e l}-\Delta G_{\text {desolv }}
$$

The membrane/protein association could generally be given as combination of nonspecific electrostatic and hydrophobic interactions. The non-polar contribution could be given in simple expression:

$$
\Delta G_{n p}=\gamma \Delta A
$$

where $\gamma$ is the surface tension coefficient and $\Delta \mathrm{A}$ is the change in solvent accessible surface area upon association. An approximation to the total nonspecific free energy of membrane association of a peripheral protein can be taken as the sum of $\Delta \mathrm{G}_{\mathrm{el}}$ and $\Delta \mathrm{G}_{\mathrm{np}}$, so that

$$
\Delta G=\Delta G_{e l}+\Delta G_{n p}
$$

The additivity of electrostatic and non-polar interactions qualitatively explains membrane binding behavior with protein. Long-range electrostatic attraction increases probability of a protein/membrane interaction and helps orient the protein. As the protein approaches the membrane surface, there are two competing short-range interactions: (1) the (repulsive) desolvation of both the protein and membrane as both, which contain charged and polar groups, begin to lose their favorable interactions with the polar aqueous solvent; and (2) the favorable non-polar partitioning of hydrophobic groups into the membrane interface as described by the interfacial hydrophobicity scale. The FDPB method, allows us to examine quantitatively how the combination of favorable electrostatic attraction, repulsive desolvation, and favorable non-polar interactions leads to different membrane binding behaviors. Each case must be considered separately since the relative contribution of each of these energetic terms will depend on the structure and composition of the membrane/protein system. 


\subsection{Materials and Methods}

\subsubsection{Experimental residual dipolar couplings}

Dipolar coupling was previously measured for the Rec-A-inactivating protein DinI dissolved in dilute suspension of Pf1 bacteriophage on $300 \mathrm{mM}$ salt concentrated ${ }^{15} \mathrm{~N}$ enriched sample [Zweckstetter, Hummer 2004]. ${ }^{15} \mathrm{~N}-{ }^{1} \mathrm{H}$ RDCs were derived from inphase/anti-phase (IPAP) $\left[{ }^{15} \mathrm{~N},{ }^{1} \mathrm{H}\right]$-heteronuclear single quantum coherence (HSQC) experiments [Ottiger, Delaglio 1998]. Alignment tensors in liquid-crystalline or paranematic Pf1 were obtained by fitting ${ }^{15} \mathrm{~N}-{ }^{1} \mathrm{H}$ RDCs to high-resolution NMR DinI (PDB code: 1GHH) [Ramirez, Voloshin 2000] structures, using singular value decomposition (SVD) as implemented in the program PALES [Zweckstetter and Bax 2000].

\subsubsection{Coordinates and Structural Models}

In our calculations high-resolution NMR structure for 81-residue RecA-inactivating protein DinI was used from the protein data bank (PDB) (PDB code: 1GHH) [Ramirez, Voloshin 2000]. Structural models for phospholipid bilayers were built as described [Peitzsch, Eisenberg 1995]. We used all-atom molecular model bilayer containing 33\% acidic lipid (2:1 PC/PS). The zwitterionic PC and negatively charged PS lipids in each leaflet, containing 192 lipids, were uniformly distributed in a hexagonal lattice. Each lipid headgroup occupies an area of $68 \AA^{2}$ in the plane of the membrane and the lipid head group regions from the two opposing membrane leaflets encompass about half the thickness of the bilayer. It is assumed that the lipids change neither structure nor position upon interaction with the protein. These approximations are not unreasonable for the protein DinI which is charged and do not penetrate the membrane interface to a large degree. Previous work has shown that the membrane 
partitioning of basic peptides that reside outside the polar envelope of the membrane is independent of whether the membrane is in the liquid crystalline or gel phase [BenTal, Honig 1996], suggesting that the use of static bilayer models is appropriate for calculating the alignment of peripheral proteins.

\subsubsection{Model of Protein/bilayer}

In the calculations described in this work, we represented the protein DinI and the lipid bilayer in atomic detail, as opposed to simplified electrostatic model in PALES, and the solvent as homogenous medium of constant dielectric. Both the Debye length and the dimension of DinI are much smaller than the radius of the vesicles so we can assume the protein DinI interact with a planar surface. Each atom of a protein/bilayer system is assigned a radius and partial charge that is located at its nucleus. The charges and radii used for the amino acids were taken from a CHARMM22 parameter set [Brooks 1983]; those used for lipids are the ones described and used in previous studies [Ben-Tal, Honig 1997;Ben-Tal, Honig 1996;Murray, Hermida-Matsumoto 1998]. The lipid bilayers are negatively charged with a total surface charge density of $1 \mathrm{e} / 204 \AA^{2}$ (33\% PS), which closely mimics the surface charge density, of -0.475 $\mathrm{e} / \mathrm{nm}^{2}$, on bacteriophage Pf1.

We built atomic models with the DinI molecules in different configurations relative to the lipid bilayers; one of them is shown in Figure 5.3. For each configuration, the protein was docked in the aqueous solution above the membrane and steric clash was avoided by manual inspection. Each configuration is determined by six coordinates: three Cartesian coordinates $(x, y, z)$ define location of the geometrical center of the protein relative to the center of the membrane section, and three angular coordinates $(\theta, \alpha, \eta)$ define its orientation with respect to the bilayer. 
The rotational angles were measured relative to the orientation of Figure 5.3. $\theta$ denotes rotations of the protein around $\mathrm{x}$-axis, the normal to the membrane surface, $\alpha$ denotes rotation of the protein around $\mathrm{z}$, an axis parallel to the membrane surface in the plane of the figure, and $\eta$ denotes rotation of the protein around $y$, an axis parallel to the membrane surface and perpendicular to the plane of the figure.

We define $\mathrm{R}$ as the minimal distance between the van der Waals surfaces of the protein and the lipid bilayer along the $\mathrm{x}$-axis, with $\mathrm{R}=0$ at van der Waals contact.

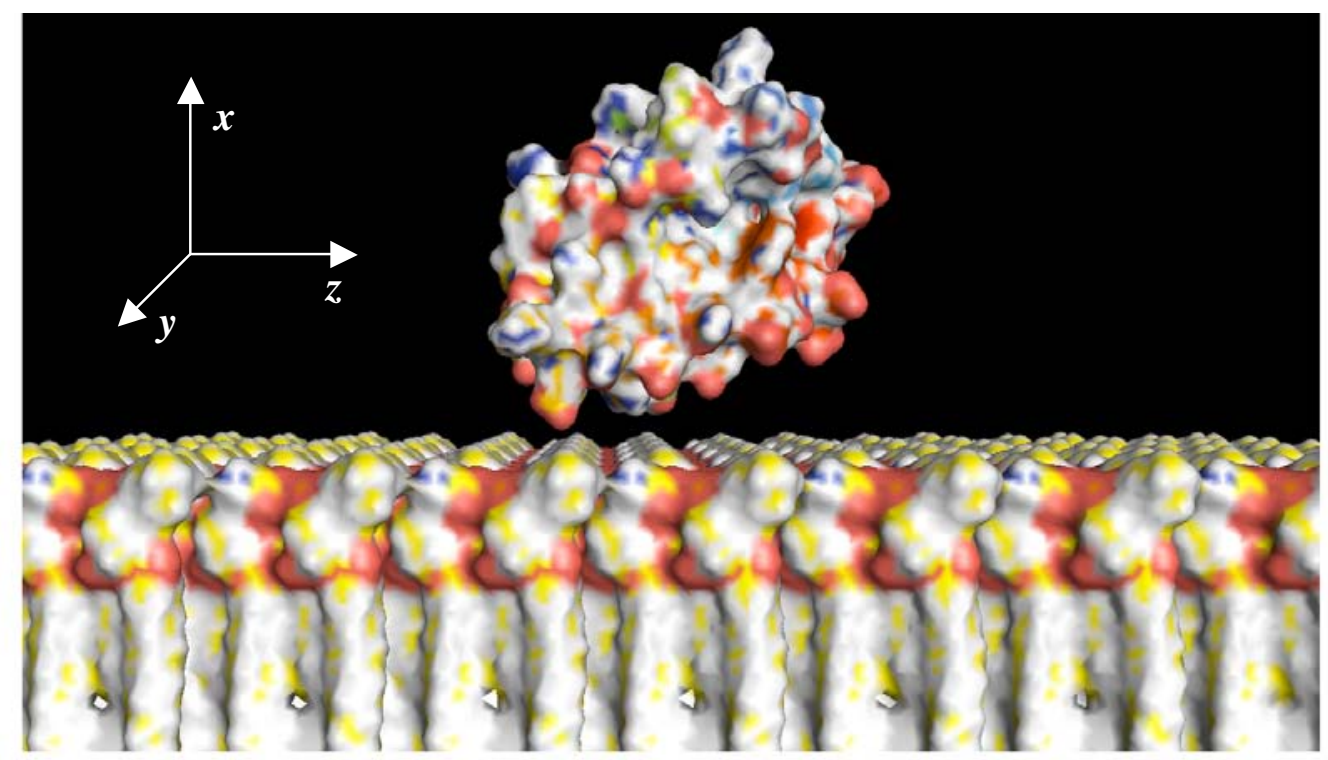

Figure 5.3: Molecular model of a portion of a PC/PS (2:1) bilayer membrane and protein DinI. The van der Waals surface of the protein and membrane are shown. The molecular surface created by rolling a sphere with the radius of water molecule, 1.4 $\AA$, on the atomic model above.

Denoting the distance and orientation dependent electrostatic free energy of interaction between a protein molecule and the membrane at a given configuration by $\mathrm{E}_{\mathrm{el}}(\mathrm{x}, \mathrm{y}, \mathrm{R}, \alpha, \eta, \theta)$ and choosing a reference state where the energy of interaction is zero when the protein is at $\mathrm{R}=\infty$, i.e., $\mathrm{E}_{\mathrm{el}}(\mathrm{x}, \mathrm{y}, \infty, \alpha, \eta, \theta)=0$, the total electrostatic free energy of interaction, $\Delta \mathrm{E}_{\mathrm{el}}$, would be calculated as given by Equation 5.1. 


\subsubsection{Alignment tensor prediction}

The Boltzmann probability $\mathrm{p}_{\mathrm{B}}$ could be calculated from the electrostatic free energy of interaction as follows,

$$
p_{B}=\exp \left[-\Delta E_{e l}(x, y, R, \alpha, \eta, \theta) / k_{B} T\right]
$$

Alignment tensor for each distance and orientation, $\mathbf{A}_{\text {protein, }}$ was then calculated using the equation below,

$$
\boldsymbol{A}_{\text {protein }}=\int \boldsymbol{A} p_{B}(x, y, R, \alpha, \eta, \theta) d V d \Omega / \int p_{B}(x, y, R, \alpha, \eta, \theta) d V d \Omega
$$

where $\mathrm{V}$ and $\Omega$ denotes the Cartesian and angular volume, respectively and the alignment matrix, A, obtained from the steric effect has its components,

$$
A_{i j}=1 / 2\left(3 \cos \theta_{i} \cos \theta_{j}-\delta_{i j}\right)(i, j=x, y, R) .
$$

\subsubsection{Electrostatic calculations}

We calculated electrostatic potentials, $\varphi(r)$, and electrostatic free energies of interaction, $E_{\mathrm{el}}(\mathrm{x}, \mathrm{y}, \mathrm{R}, \alpha, \eta, \theta)$, for each of the 122 configurations from a modified version of the DELPHI program [Gallagher and Sharp 1998], which is adapted to solve the nonlinear Poisson-Boltzmann equation for protein/membrane systems [BenTal, Honig 1996;Peitzsch, Eisenberg 1995]. DELPHI produces finite-difference solutions to the Poisson-Boltzmann equation on a cubical lattice for a system where the solvent is described in terms of a bulk dielectric constant and mean concentrations of mobile ions/atoms, while the protein and the lipids comprising the bilayer are described in terms of the coordinates of the individual atoms as well as their atomic radii and partial charges.

The protein DinI and membrane model were then mapped onto a threedimensional cubic lattice of $257^{3}$ points, each of which represents a small region of 
the peptide, membrane or solvent. The molecular surfaces of the DinI and the membrane are defined as the point of contact between a spherical probe with the radius of a water molecule $(1.4 \AA)$ and the van der Waals surface. The spaces enclosed by the molecular surfaces, or interior regions, were assigned a low dielectric constant of 2. The space outside the molecular surfaces, or the exterior region, was assigned a high dielectric constant of 80 [Ben-Tal, Honig 1996]. We solved the nonlinear Poisson-Boltzmann equation in the finite-difference approximation, for each distance and orientation of the protein DinI and the membrane and calculated $\mathrm{E}_{\mathrm{el}}(\mathrm{x}, \mathrm{y}, \mathrm{R}, \alpha, \eta, \theta)$ at this configuration from the spatial distribution of the charges and the electrostatic potential. Electrostatic free energies are obtained from the calculated potentials, and the electrostatic free energy of interaction is determined as the difference between the electrostatic free energy of the protein DinI in a specific orientation with respect to the membrane surface and the electrostatic free energies of the DinI and membrane infinitely far apart, i.e., taken separately, as given by Equation 5.2. The numerical calculation of the potential is iterated to convergence, which is defined as the point at which the potential changes less than $10^{-5} \mathrm{kT} / \mathrm{e}$ between successive iterations.

A sequence of focusing runs of increasing resolution was employed to calculate the electrostatic potentials (e.g., $0.375,0.75,1.5$ and $3.0 \mathrm{grid} / \AA$ ). In the initial calculation, the DinI/membrane model encompassed a small percentage of the lattice (10\%), and the potentials at the boundary points of the lattice are approximately zero; this procedure ensures that the system is electro-neutral. The calculations with atomic models of membranes were performed in a final resolution of 3 grid $/ \AA$ and lattice sizes of $257^{3}$ were used. We also tested the convergence of the results with respect to the lattice size and scale: increasing the grid box from $257^{3}$ to $321^{3}$ (at a constant scale 
of 3 grids/Å) alters the electrostatic free energy of interaction of DinI with a 2:1 PC/PS membrane in $300 \mathrm{mM}$ monovalent salt by $<0.1 \mathrm{kcal} / \mathrm{mol}$ (Data not shown). The convergence with respect to lattice scale depends on the distance between the protein and membrane and on their relative orientation. The results obtained using a grid box of $257^{3}$ and scales of $0.5,1.0$ and 2.0 grids/ $\AA$ differed by only $<0.3 \mathrm{kcal} / \mathrm{mol}$ (Data not shown) and the depth of the minimum in the electrostatic free energy, which dominates the electrostatic component of the binding free energy, changed by only $<0.2 \mathrm{kcal} / \mathrm{mol}$ (Data not shown). The precision in the electrostatic free energies of interaction, determined as the difference between the results obtained at the two highest resolution scales, is $0.3 \mathrm{kcal} / \mathrm{mol}$ for all calculations. 


\subsection{Results and Discussions}

\subsubsection{Calculation of electrostatic free energies}

In this study, the simplistic electrostatic model as described previously [Zweckstetter, Hummer 2004], was replaced by an atomistic detailed electrostatic effect to predict the alignment tensor of the protein DinI. 2:1 PC/PS Lipid bilayer was used to mimic the charge density of infinite cylinder (Pf1 bacteriophage), $-0.5 \mathrm{e} / \mathrm{nm}^{2}$. The center of gravity of the van der Waals surface of the protein DinI is moved on a onedimensional grid (along the $\mathrm{x}$-axis, the normal to the bilayer surface), with a grid spacing between grid points of $0.5 \AA$ and $1 \AA$, away from the surface of the lipid bilayer. The molecule was rotated only in the y-z plane to sample 122 orientations on a unit sphere, as determined by a double cubic lattice method, as it provides a highly uniform sampling [Eisenhaber 1995] and the $20^{\circ}$ rotation around the $\mathrm{x}$-axis was not performed, as that would mean solving non-linear Poisson-Boltzmann equation for 2196 configurations, which is computationally very expensive. Therefore, for all results presented in this study, only 122 orientations were used.

Figure 5.4 shows the one (mean) of the 122 orientations of the DinI in front of the phospholipid bilayer. Phospholipid bilayer lies in the y-z plane and protein is translated along the $\mathrm{x}$-axis, normal to the bilayer surface. In the simplified electrostatic model, for each orientation of the protein, with respect to the membrane surface, nonexcluded alignment matrix was calculated according to Equation 5.9. Each nonexcluded matrix was weighed according to its Boltzmann probability after calculating the electrostatic potential from the simplified electrostatic model [Zweckstetter, Hummer 2004]. In this model, the electrostatic free energy in Equation 5.7 was replaced by the solution to the nonlinear Poisson-Boltzmann equation calculated with full atomistic detail. 
The distance and orientation dependent electrostatic free energy was calculated as discussed in Materials and Methods. Figure 5.4 shows one (mean) of the 122 orientations of the DinI in front of the phospholipid bilayer. The most attractive orientation should have the maximum number of positive charges facing the lipid bilayer because binding is driven mainly by electrostatic interactions. The positive

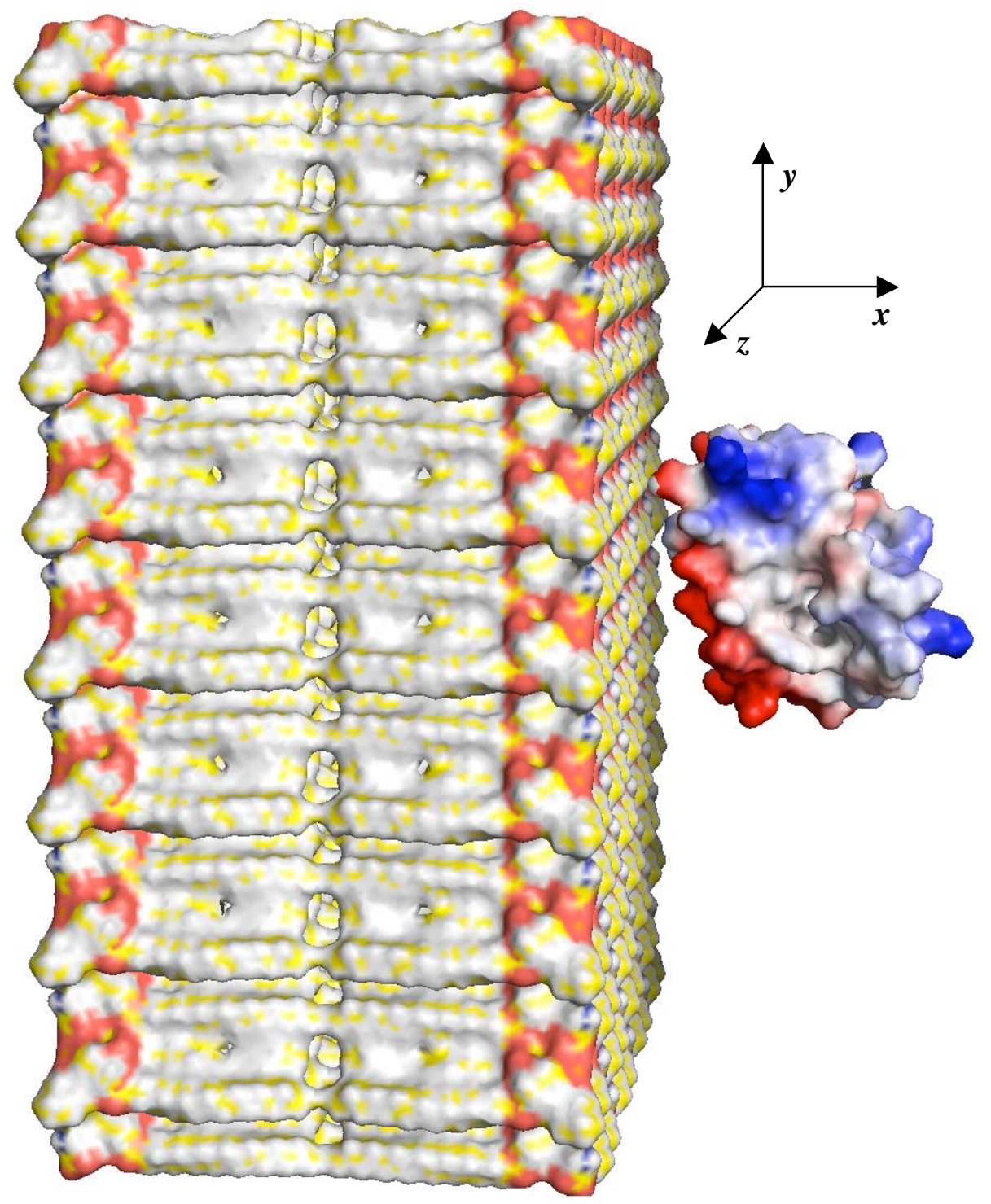

Figure 5.4: Model of the alignment of protein. One of the 122 orientations of protein DinI at the surface of a 2:1 PC/PS bilayer in $300 \mathrm{mM}$ salt is shown. Lipid bilayer mimics the Pf1 surface's charge density of $-0.5 \mathrm{e} / \mathrm{nm}^{2}$. Van der Waals surface of the bilayer and the electrostatic surface of the protein are shown. Lipid bilayer is oriented with the normal to its surface along the $\mathrm{x}$-axis. The minimal distance between the van der Waals surfaces of the protein and the membrane in this configuration is $R=2 \AA$. 
potential due to the protein extends to the surface of the bilayer, accounting for the strong favorable electrostatic interaction. Some of the acidic groups on the protein are clearly repelled by the bilayer (e.g., regions of negative potential in the protein). Though suggestive, the figure clearly indicates the limitations of qualitative analysis. There is no simple way, for example, to know the relative magnitudes of the contribution of different regions of the protein. Also, there would be different contributions to the electrostatic free energy of interactions such as Coulombic, nonpolar etc [Ben-Tal, Honig 1997;Murray, Arbuzova 2002]. This would be due to the fact that, when the protein approaches the membrane surface, the charges on the protein and the membrane are transferred to a low dielectric region, which would give rise to Born repulsion (desolvation effects).

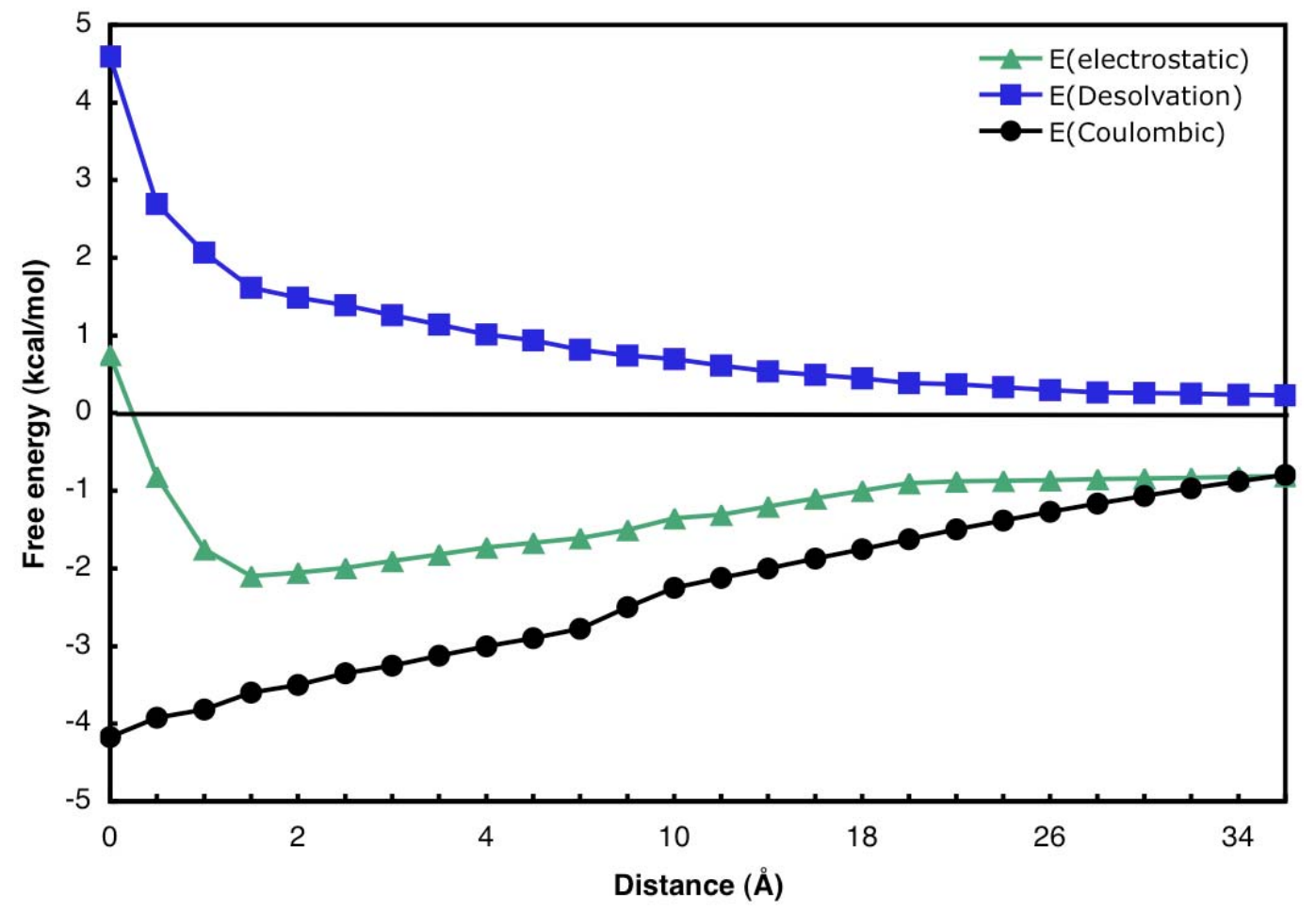

Figure 5.5: Electrostatic free energy of interaction for the orientation, shown in Figure 5.4, between DinI and a 2:1 PC/PS bilayer in $300 \mathrm{mM}$ salt. Free energy of interaction is plotted as a function of distance between van der Waals surfaces of DinI and the membrane; Black circles represent the long-range Coulombic attraction. Blue squares represent the unfavorable short-range desolvation repulsion, and the balance between the long-range Coulombic and short-range desolvation repulsion results in an electrostatic free energy minimum (green triangles) when the protein and membrane separated by a layer of water (refer Equation 5.4). 
Figure 5.5 plots the electrostatic free energies of interaction for DinI with a 2:1 PC/PS bilayer in $300 \mathrm{mM}$ salt as a function of $\mathrm{R}$, the distance between the surfaces of the protein and membrane. As depicted by the curves, the electrostatic free energy becomes increasingly attractive as the protein approaches the membrane surface but is highly repulsive at short distances. The long-range attraction is due to Coulombic interactions between negatively charged membrane and the positively charged surface of the protein, while the repulsion is due principally to the removal of water from the charged surfaces of the protein and membrane at short distances (desolvation effects) but also repulsive Coulombic interactions between negatively charged groups on the protein and membrane. The combination of these opposing effects - Coulombic attraction versus Coulomb and desolvation repulsions - results in a minimum in the electrostatic free energy at $\mathrm{R} \sim 2.0 \AA$, the diameter of a water molecule. The orientation corresponding to the minimum in the free energy is depicted in Figure 5.4.

\subsubsection{Prediction of molecular alignment}

To compare the detailed electrostatic calculation with the simplified electrostatic model [Zweckstetter, Hummer 2004], we predicted the alignment tensor from the electrostatic free energy of interaction, as obtained by solving full nonlinear PoissonBoltzmann equation, and compared with the alignment tensor obtained from simplified model. Different contributions (as from Figure 5.5) were also taken into account to predict the alignment tensor.

Experimental RDCs have been measured for protein DinI and the RDCs fit very well with the high-resolution NMR structure of DinI molecule, when evaluated by SVD, with a Pearson's linear correlation coefficient, $\mathrm{R}_{\mathrm{p}}$, of 0.985 [Zweckstetter, Hummer 2004]. ${ }^{15} \mathrm{~N}-{ }^{1} \mathrm{H}$ dipolar couplings $\left({ }^{1} \mathrm{D}_{\mathrm{NH}}\right)$ predicted on the basis of a purely 
steric obstruction effect and simplified electrostatic model correlate with the experimentally observed RDCs, with the correlation coefficient of 0.34 and 0.96 , respectively, with DinI molecule. This already indicates that in highly charged liquidcrystalline media, such as bacteriophage Pf1, interactions other than steric obstruction are important for molecular alignment and inclusion of electrostatic interactions, treated in the highly simplified manner improved the prediction of RDCs and the magnitude of alignment, dramatically. Pearson's correlation coefficient of 0.96 was obtained with large number of (2196) orientations. Since the atomistic models require more computation time, much less, namely 122 could be calculated. When the number of orientations 122 and 1 was considered, the simplified model, as implemented in PALES [Zweckstetter, Hummer 2004], yielded only a correlation coefficient of 0.78 and 0.57 , respectively.

\section{Prediction of alignment from the minimum energy orientation}

Detailed electrostatic calculations were done to predict the residual dipolar couplings from the minimum energy orientation (mean orientation). Figure 5.5 represents the minimum electrostatic free energy of interaction between protein DinI and lipid bilayer. In that particular (mean) orientation, for each of the distance along the $\mathrm{x}$-axis in front of the lipid bilayer, the nonexcluded matrix A, which was obtained within PALES, was weighted according to its Boltzmann probability, obtained from the detailed electrostatic calculations (the total electrostatic free energy of interaction (Figure 5.5) was used to calculate the alignment tensor and the RDCs as by Equation 5.8). Saupe order matrix was obtained from the weighted nonexcluded alignment

matrix. For the 81-residue rec-A-binding protein DinI, experimental ${ }^{1} \mathrm{D}_{\mathrm{NH}}$ values correlated with the values predicted from the Saupe ordered matrix (calculated from the electrostatic free energies for the mean orientation by atomistic model) with a 


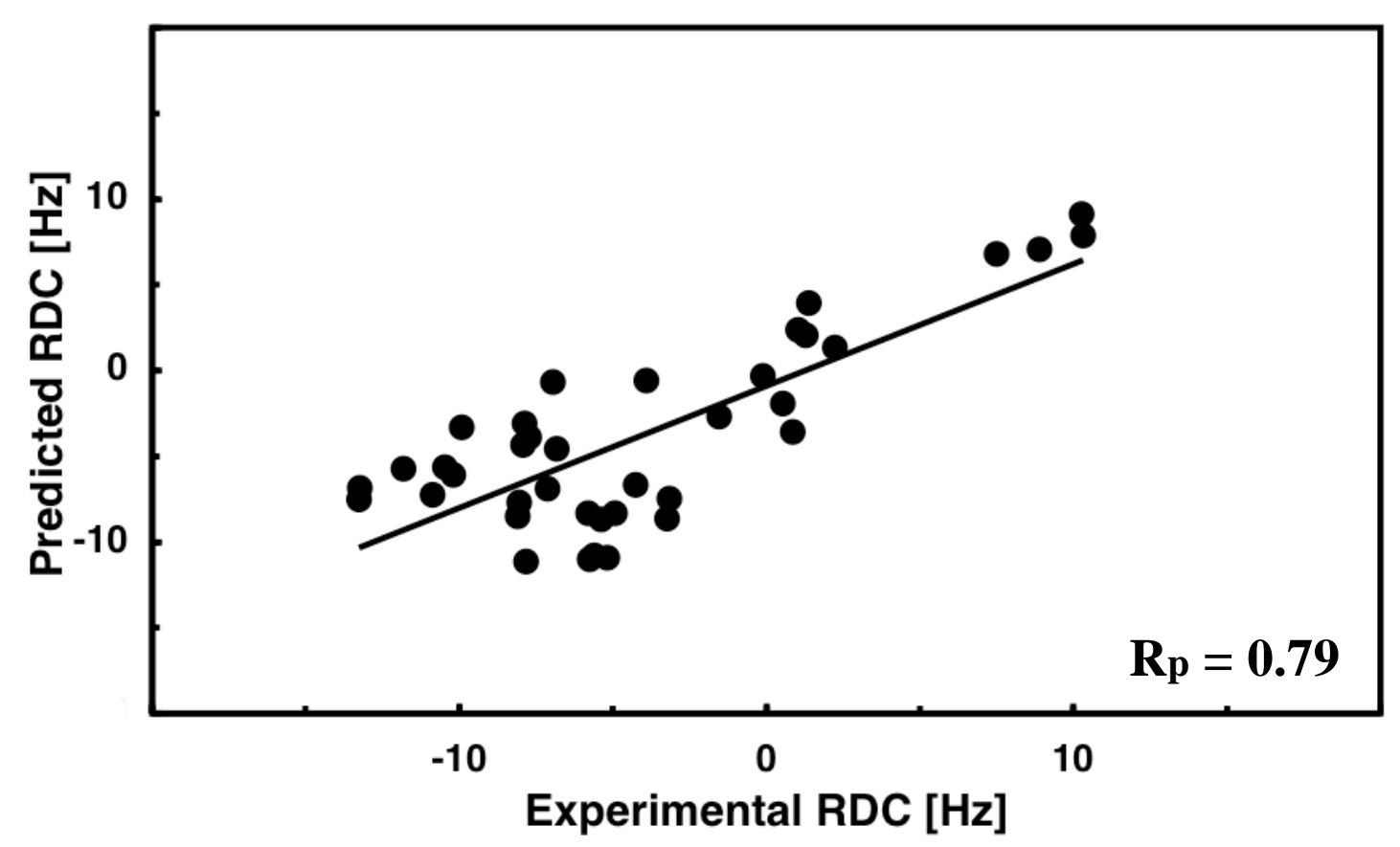

Figure 5.6: Comparison of experimental ${ }^{1} \mathrm{D}_{\mathrm{NH}}$ values measured at $300 \mathrm{mM}$ salt with values predicted from the total minimum electrostatic free energy configuration as in Figure 5.4.

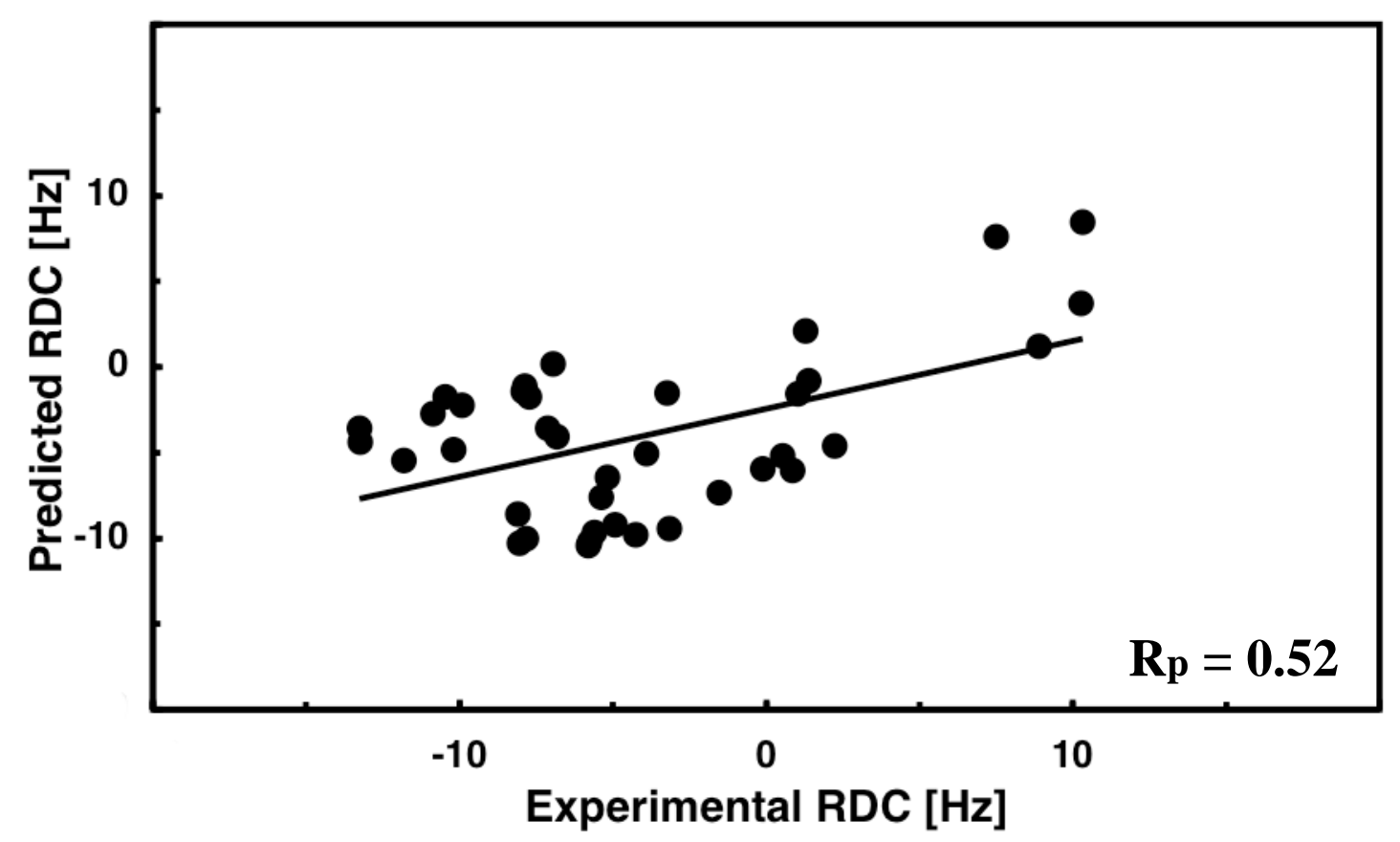

Figure 5.7: Comparison of experimental ${ }^{1} \mathrm{D}_{\mathrm{NH}}$ values measured at $300 \mathrm{mM}$ salt with values predicted, based only on the Coulombic term, from the total minimum electrostatic free energy configuration as in Figure 5.4. 
Pearson's correlation coefficient $\mathrm{R}_{\mathrm{p}}=0.79$ (Figure 5.6), whereas, the correlation coefficient obtained from the simplistic electrostatic model was 0.57 (by sampling only one orientation), as compared to the 2196 orientations that obtained correlation factor of 0.96 [Zweckstetter, Hummer 2004]. To take into account only the contributions from the electrostatic (Coulombic) effect, the desolvation term was removed from the total electrostatic free energy of interaction as by Equation 5.3 and Equation 5.4. Boltzmann probability obtained only from the Coulombic energy was used to predict the dipolar couplings. The correlation between the experimental and predicted RDCs decreased to 0.52 (Figure 5.7), suggesting that the Coulombic term needs the compensation by the water exclusion term.

\section{Prediction of alignment from 122 Orientations}

Accuracy of the predictions depend also on the total number of conformations sampled adjacent to the membrane surface. Hence detailed electrostatic calculations were also done by increasing the number of conformational samplings to 122 , opposed to restricting to only for the mean orientation as discussed above. Here, for each of the distance along the $\mathrm{x}$-axis and for each of the 122 orientation of the protein with respect to the lipid bilayer in $y-z$ plane, the nonexcluded matrix A, which was obtained within PALES, was weighted according to its Boltzmann probability, obtained from the detailed electrostatic calculations for 122 orientations. Saupe order matrix was obtained from the weighted nonexcluded alignment matrix. For the 81residue rec-A-binding protein DinI, experimental ${ }^{1} \mathrm{D}_{\mathrm{NH}}$ values correlated with the values predicted from the Saupe ordered matrix with a Pearson's correlation coefficient $R_{p}=0.92$ (Figure 5.8). Clearly, when we compare to the results above ( 0.79 for mean orientation) the large sampling yielded better correlation. In addition, the predicted magnitude of alignment, $G_{m a g}$, was approximately five times less than 


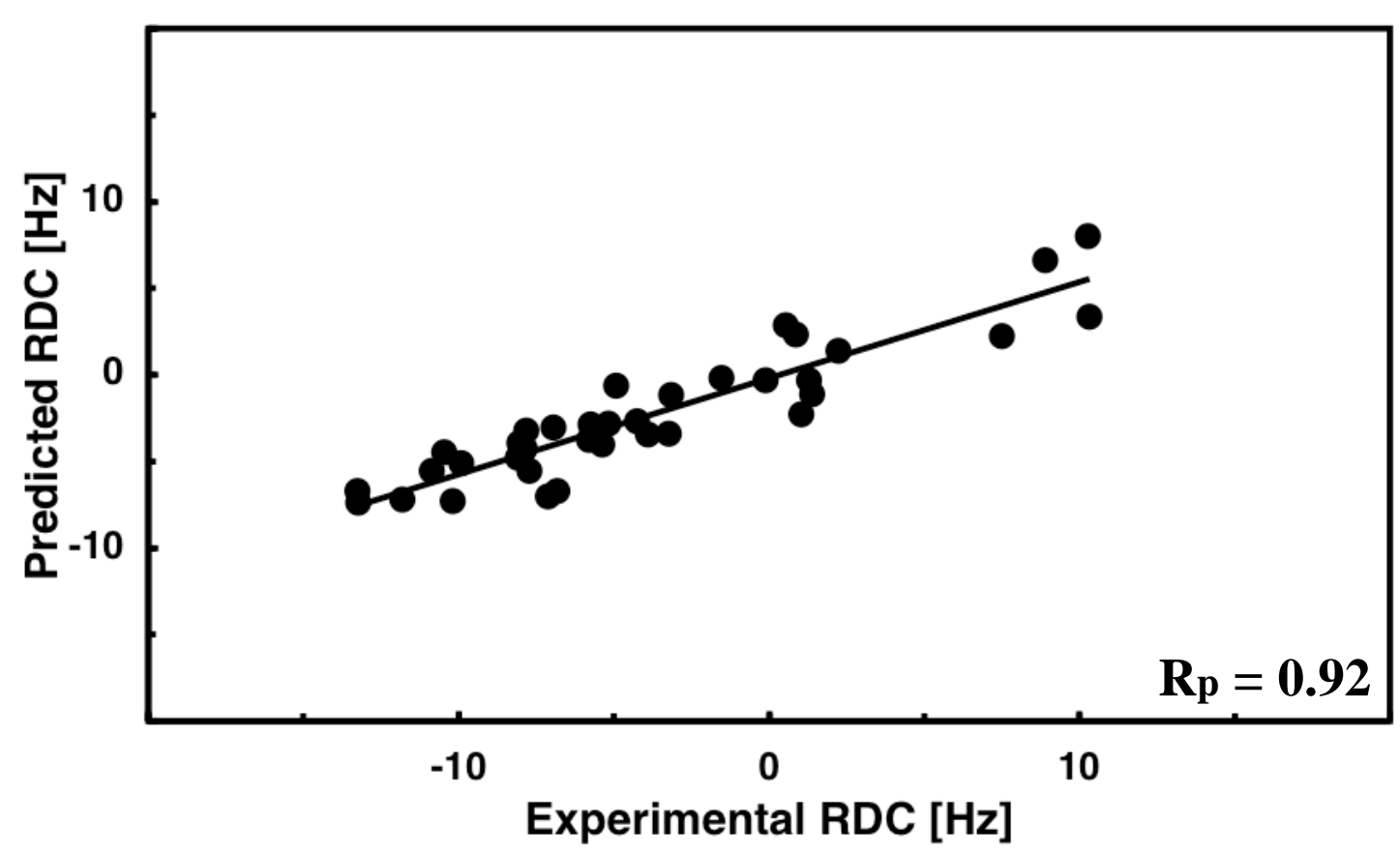

Figure 5.8: Comparison of experimental ${ }^{1} \mathrm{D}_{\mathrm{NH}}$ values measured at $300 \mathrm{mM}$ salt with values predicted on the basis of the molecule's three-dimensional shape and atomistic electrostatic effects for protein DinI, considering 122 different orientations.

the experimental value of $4.1 \times 10^{-3}$.

To take into account only the contributions from the electrostatic (Coulombic) effect, the desolvation term was removed from the total electrostatic free energy of interaction as by Equation 5.3 and Equation 5.4. Boltzmann probability obtained from the Coulombic energy was used to predict the residual dipolar couplings. The correlation between the experimental and predicted RDCs decreased to 0.82 (shown in Figure 5.9). Thus supporting the fact that the short-range repulsion term contributes to the accuracy in the prediction of residual dipolar couplings. It is expected that, by sampling 2196 orientations in the detailed electrostatic model, the predictions should improve when compared to the simplified model. In addition, the predicted magnitude of alignment, $\mathrm{G}_{\mathrm{mag}}$, was $1.25 \times 10^{-3}$, which was three times less than the experimental magnitude of alignment, whereas the alignment magnitude predicted by electrostatic PALES was $7.97 \times 10^{-3}$. Inaccuracies in the prediction of magnitude of alignment 
could be arising from the fact, that the translational grid was under sampled, that is only up to $30 \AA$.

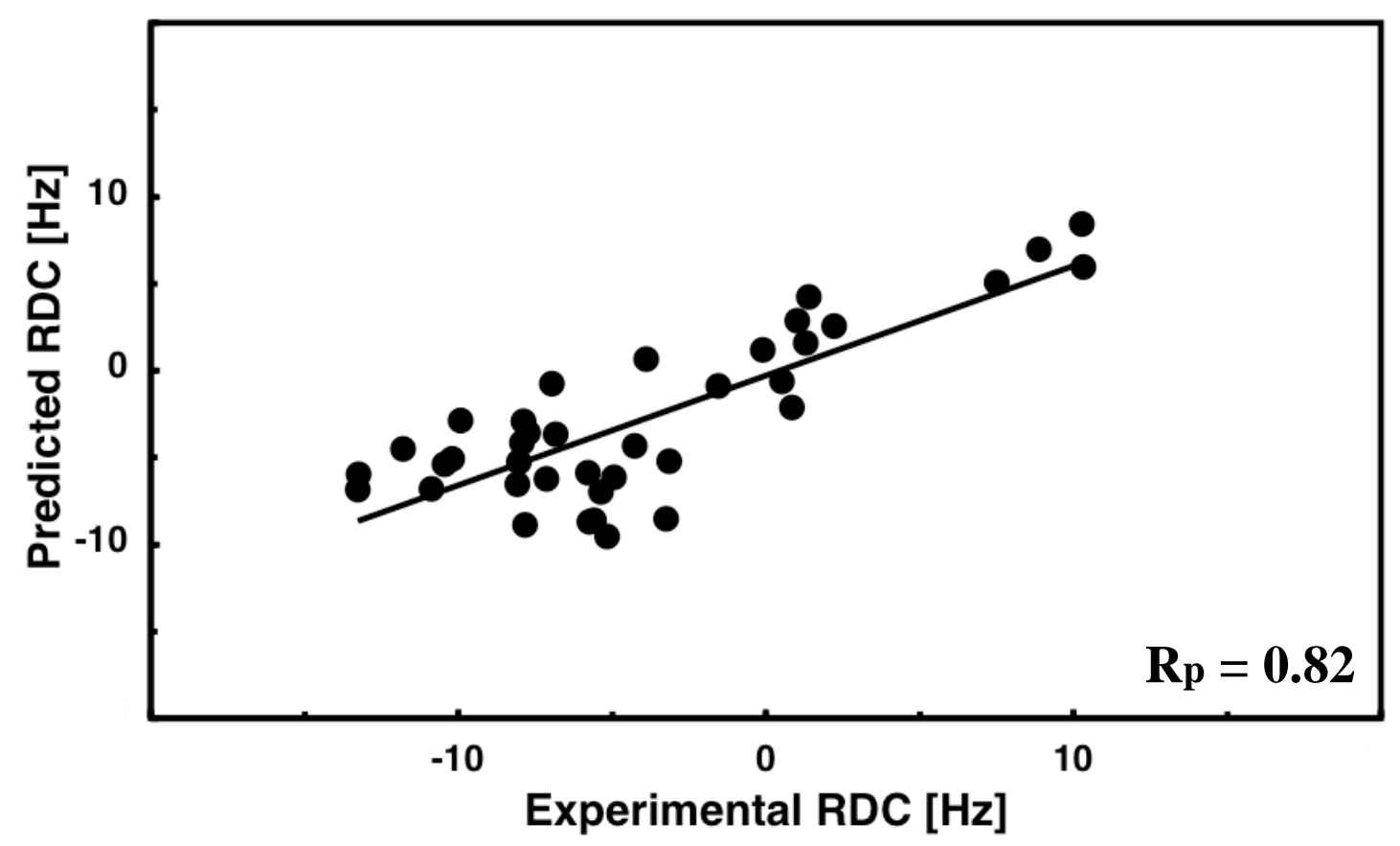

Figure 5.9: Comparison of experimental ${ }^{1} \mathrm{D}_{\mathrm{NH}}$ values measured at $300 \mathrm{mM}$ salt with values predicted, considering only the Coulombic contribution from the total electrostatic free energy, on the basis of the molecule's three-dimensional shape and atomistic electrostatic effects for protein DinI, considering 122 different orientations.

\section{Prediction of magnitude of alignment}

For the simplified, model, the predicted alignment magnitudes deviated significantly for salt concentrations below $0.01 \mathrm{M}$ and above $0.3 \mathrm{M}$ from experimental values [Zweckstetter, Hummer 2004]. Hence, to investigate the accuracy of the detailed model, the magnitude of alignment was predicted by repeating the detailed electrostatic calculations for the protein in the mean orientation at salt concentrations ranging between $0.03-0.4 \mathrm{M}$. Minimum electrostatic free energy obtained at various salt concentrations were converted to Boltzmann factor (according to equation 5.7) to obtain the binding factor, B. Figure 5.10 shows the overlay of $\mathrm{G}_{\mathrm{mag}}$ as obtained from experimental dipolar couplings by SVD (blue lines) and B values (black lines) as 


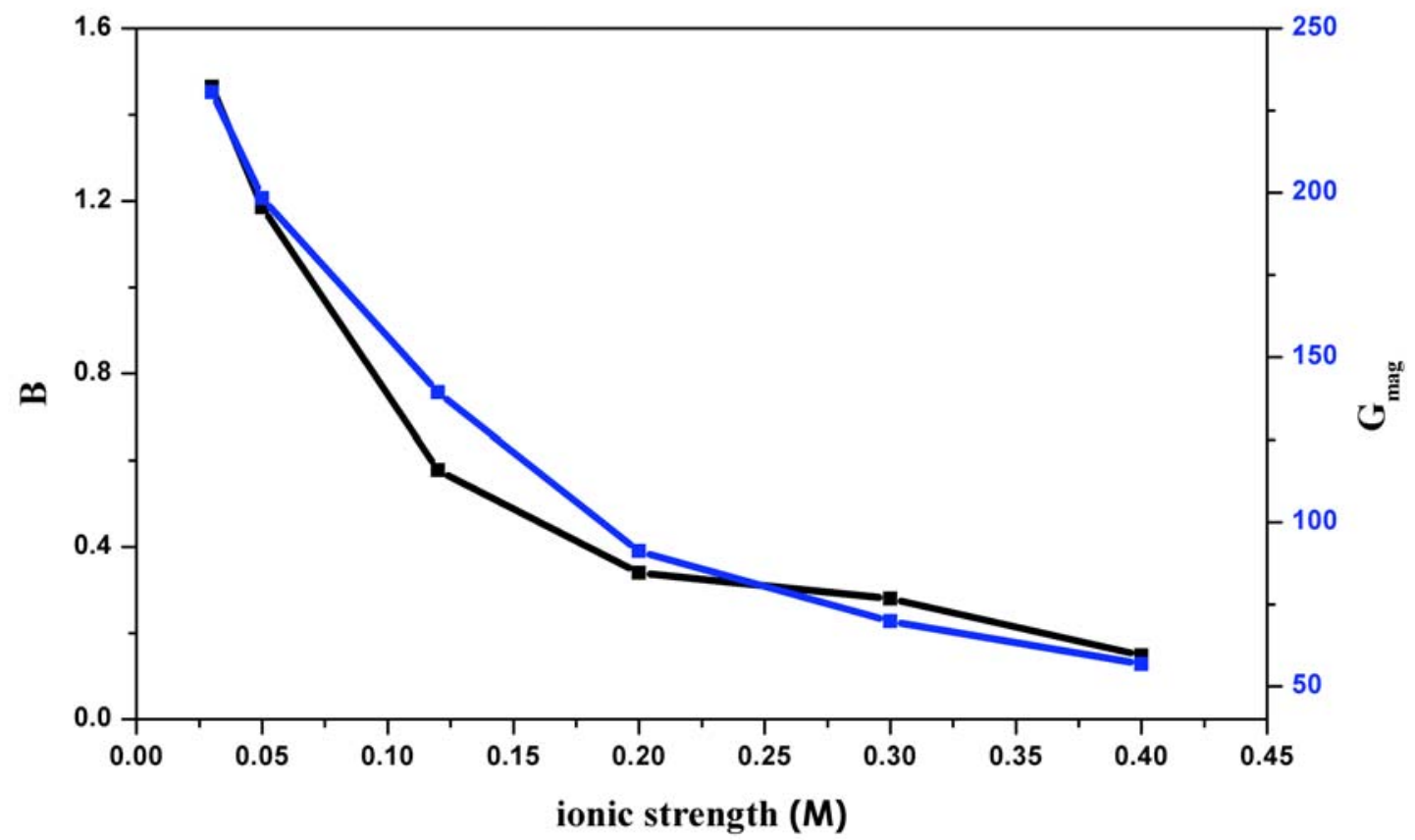

Figure 5.10: Comparison of experimental alignment magnitude $\left(\mathrm{G}_{\mathrm{mag}}\right)$, obtained by SVD, (blue lines) with B values predicted from atomistic model (black lines).

predicted from detailed electrostatic model. The overall profile obtained from the detailed model is comparable to the experimental values, as opposed to the simplified model. Thus, the preliminary results suggests that the presence of desolvation term in the detailed model already improves the accuracy of prediction at different salt concentrations and future works are planned to perform the similar calculations for different systems reported in Zweckstetter, Hummer 2004.

The study presented here introduced a possible extension to the existing simplistic electrostatic model used in PALES, by adding more detailed electrostatic effects. Previous model approximated the electrostatic interaction between a solute and an ordered phage particle as that between the solute's surface charges and the electric field of the phage [Zweckstetter, Hummer 2004]. This simplified geometry allowed one to obtain an analytical expression for the energy of interaction at each protein-phage distance in terms of Gouy-Chapman-Stern theory [Chapman 1913;Gouy 1910]. Here, in this method the alignment medium (here lipid bilayer) and 
the protein (DinI) were represented in atomic detail. The reciprocal effects of the protein and the membrane on each other's electrostatic potentials, which were ignored in the previous model, are taken into account and give rise to a Born repulsion between the protein and the membrane at a short distance. The short-range repulsion, when added to the long-range coulombic attraction yields a minimum in the electrostatic free energy of attraction between the membrane and the protein.

Table 5.1: Table representing the correlations obtained using two different electrostatic models.

\begin{tabular}{lcc}
\hline \multicolumn{1}{c}{ Electrostatic Model } & \multicolumn{2}{c}{ Correlation $\left(\mathrm{R}_{\mathrm{p}}\right)$} \\
& $\begin{array}{c}\text { Mean } \\
\text { Orientation }\end{array}$ & $\begin{array}{c}122 \\
\text { Orientations }\end{array}$ \\
\hline Simplistic electrostatic model (PALES) & $0.57^{\S}$ & $0.78^{\S}$ \\
Detailed electrostatic model & 0.52 & 0.82 \\
$\quad$ Only Coulombic & 0.79 & 0.92 \\
Coulombic + short range Born-repulsion & & \\
\hline
\end{tabular}

[§] When performed for 2196 orientations, correlation coefficient was 0.96 [Zweckstetter, Hummer 2004].

The simplified electrostatic model predicted correctly not only the RDCs but also the magnitude of alignment for protein DinI [Zweckstetter, Hummer 2004], which implies other attractive nonpolar (e.g. hydrophobic) interactions between the protein and the membrane may compensate for Born-repulsion. The prediction of the detailed atomic model, which considers the Born-repulsion effects and the influence of protein on the bilayer, was comparable to the simplified model and reasonably accurate when the same number of orientations was assumed (Table 5.1). However, since the simplified model is extremely fast, more orientations could be considered so that it still represents the standard for accuracy given a certain amount of time. When we use an atomistic model that assumes purely electrostatic (Coulombic) effect, the predicted alignment tensors for DinI was further away from the observed values $(0.52$ 
and 0.82 , respectively for the mean and 122 orientations), but was similar to the values obtained from simplified model ( 0.57 and 0.78 , respectively), for the given number of orientations (Table 5.1). The quality of the predictions obtained by considering full electrostatic free energy (Coulombic and desolvation effects) was better compared to the predictions obtained from the simplistic electrostatic model, for the given number of orientations (Table 5.1). Sampling large number of orientations of proteins adjacent to the membrane surface is expected to further improve the accuracy of the predictions of alignment tensor and residual dipolar couplings. Also, the calculations here were restricted up to a translation grid of $\sim 30 \AA$, unlike in electrostatic PALES, where it was sampled until the potential reduces to the range of 1e-06 (which is nearly $\sim 85 \AA$ ). Increasing the translations grid would help in improving the accuracy in predictions of magnitude of alignment.

In addition, addressing the following concerns in the detailed model could lead to better accuracy in the predictions.

1) The detailed model ignores some attractive interactions. This attractive interaction could arise from nonpolar contributions to the binding of protein/membrane system. Assuming the nonpolar interactions are proportional to the water-accessible surface area [Ben-Tal, Honig 1997], the water-accessible surface area would decrease as the protein approaches the bilayer, which will give rise to short-range attraction.

2) The lower correlations in the detailed model could also arise from either structural changes in the bilayer that occur on binding or some other deficiency in the model, as reported previously [Ben-Tal, Honig 1997;Zhou, Schulten 1995;Woolf, Roux 1996]. The "soft" or nonrigid nature of the bilayer surface may strongly affect the calculations of the short-range Born repulsions and nonpolar attractions, but 
should have a much smaller effect on the calculation of the long-range Coulomb attraction.

3) Deficiencies could also relate to the accuracy of the electrostatic potential defined by the membrane bilayer model used here compared to the electrostatic potential experienced by a protein in front of the bacteriophage Pf1.

4) It is also well known that the Coulomb energy does not depend on the set of atomic partial or real charges and radii used for the calculations [Ben-Tal, Honig 1996]. But the Born repulsion does depend strongly on parameters used for the calculations, i.e., the set of atomic partial charges and radii used in the Born calculations. 


\subsection{Conclusions}

In this study, a more detailed electrostatic model was used in order to improve the quality of prediction of the alignment tensor of a protein from its known threedimensional structure. To this aim, Non-linear Poisson-Boltzmann equation was solved for the protein/bilayer system, where protein and lipid bilayer where represented in atomistic detail. Calculated electrostatic free energies were used to compute molecular alignment tensors. Residual dipolar couplings predicted from the total electrostatic free energies correlated reasonably well with the experimental values, for the Rec A binding protein DinI dissolved in Pf1 bacteriophage. Preliminary result suggests that, the prediction of charge-induced molecular alignment could be improved by employing detailed electrostatic model. Contributions from the short-range Born repulsion to the total electrostatic free energy seemed crucial when the protein approaches the bilayer surface, indicating that apart from the steric interactions, short-range interactions arising from desolvation effect should also be taken into account to increase the accuracy of the prediction of alignment tensor and residual dipolar couplings for a protein. Influence of the protein on the bilayer is taken into these calculations, through both short-range interactions and long-range electrostatic effects, thus, overcoming one of the major limitations of the simple electrostatic model currently used in PALES. Though tests should be done for all the 2196 orientations of DinI molecule and for different systems at various ionic strengths. Also, from these studies, it will be possible to determine how much charge (positive/negative) is required to improve the molecular alignment of a protein for a given medium and also the correlation between the predicted and observed residual dipolar couplings over the simplified electrostatic model. 


\subsection{Outlook}

1) It was reported previously that, the simplistic electrostatic model under or over estimates the magnitude of alignment tensor at low ionic strengths in few cases. Hence, to evaluate the scaling of the magnitude of the alignment as a function of ionic strength, calculations must be done for different members of the ensemble of different protein structures (such as Ubiquitin, GB1, GB§, Dickerson dodecamer) and for many different configurations (all 2196 orientations, i.e., 122 orientations for every $18^{\circ}$ around the $\mathrm{x}$-axis) of the protein adjacent to the membrane surface. This test is also expected to address the concern related to dependence of quality of the available input structure on evaluating the molecular alignment in the simplified electrostatic model.

2) Instead of lipid bilayers, a more realistic model of the bacteriophage could also be used for the electrostatic calculations. A cylindrical surface with atomistic detail could be modeled with DELPHI to mimic the shape and surface charge density of the Pf1 Bacteriophage. Mimicking also the nonuniform electrostatic potential experienced by the protein along the z-axis of the Pf1. 


\section{Bibliography}

Abbott, A., (2005) Protein structures hint at the shape of things to come. Nature. $7042,435,547$.

Aiyar, J., Withka, J. M., Rizzi, J. P., Singleton, D. H., Andrews, G. C., Lin, W., Boyd, J., Hanson, D. C., Simon, M., Dethlefs, B., Lee, C. L., Hall, J. E., Gutman, G. A., Chandy, K. G., (1995) Topology of the Pore-Region of a K+ Channel Revealed by the Nmr-Derived Structures of Scorpion Toxins. Neuron. 5, 15, 1169-1181.

Al-Hashimi, H. M., Valafar, H., Terrell, M., Zartler, E. R., Eidsness, M. K., Prestegard, J. H., (2000) Variation of molecular alignment as a means of resolving orientational ambiguities in protein structures from dipolar couplings. Journal of Magnetic Resonance. 2, 143, 402-406.

Andrew, E. R., Bradbury, A., Eades, R. G., (1958) Nuclear Magnetic Resonance Spectra from a Crystal rotated at High Speed. Nature. 4650, 182, 1659-1659.

Andronesi, O. C., Pfeifer, J. R., Al-Momani, L., Ozdirekcan, S., Rijkers, D. T., Angerstein, B., Luca, S., Koert, U., Killian, J. A., Baldus, M., (2004) Probing membrane protein orientation and structure using fast magic-angle-spinning solid-state NMR. Journal of Biomolecular NMR. 3, 30, 253-265.

Baker, N. A., Mccammon, J. A., (2003) Electrostatic interactions. Methods Biochem Anal. 44, 427-440.

Baldus, M., (2002) Correlation experiments for assignment and structure elucidation of immobilized polypeptides under magic angle spinning. Progress in Nuclear Magnetic Resonance Spectroscopy. 1-2, 41, 1-47.

Baldus, M., (2007) ICMRBS founder's medal 2006: Biological solid-state NMR, methods and applications. Journal of Biomolecular NMR. 1, 39, 73-86.

Barrientos, L. G., Dolan, C., Gronenborn, A. M., (2000) Characterization of surfactant liquid crystal phases suitable for molecular alignment and measurement of dipolar couplings. Journal of Biomolecular NMR. 4, 16, 329-337.

Barrientos, L. G., Louis, J. M., Gronenborn, A. M., (2001) Characterization of the cholesteric phase of filamentous bacteriophage fd for molecular alignment. Journal of Magnetic Resonance. 1, 149, 154-158.

Bax, A., (2003) Weak alignment offers new NMR opportunities to study protein structure and dynamics. Protein Science. 1, 12, 1-16.

Bax, A., Grzesiek, S., (1993) Methodological Advances in Protein Nmr. Accounts of Chemical Research. 4, 26, 131-138. 
Bayrhuber, M., Graf, R., Ferber, M., Zweckstetter, M., Imperial, J., Garrett, J. E., Olivera, B. M., Terlau, H., Becker, S., (2006) Production of recombinant Conkunitzin-S1 in Escherichia coli. Protein Expr Purif. 2, 47, 640-644.

Bayrhuber, M., Vijayan, V., Ferber, M., Graf, R., Korukottu, J., Imperial, J., Garrett, J. E., Olivera, B. M., Terlau, H., Zweckstetter, M., Becker, S., (2005) Conkunitzin-S1 is the first member of a new Kunitz-type neurotoxin family. Structural and functional characterization. Journal of Biological Chemistry. $25,280,23766-23770$.

Ben-Tal, N., Honig, B., Miller, C., Mclaughlin, S., (1997) Electrostatic binding of proteins to membranes. Theoretical predictions and experimental results with charybdotoxin and phospholipid vesicles. Biophys Journal. 4, 73, 1717-1727.

Ben-Tal, N., Honig, B., Peitzsch, R. M., Denisov, G., Mclaughlin, S., (1996) Binding of small basic peptides to membranes containing acidic lipids: theoretical models and experimental results. Biophys Journal. 2, 71, 561-575.

Berendsen, H. J. C., Postma, J. P. M., Vangunsteren, W. F., Dinola, A., Haak, J. R., (1984) Molecular-Dynamics with Coupling to an External Bath. Journal of Chemical Physics. 8, 81, 3684-3690.

Bewley, C. A., (2001) Rapid validation of the overall structure of an internal domainswapped mutant of the anti-HIV protein cyanovirin-N using residual dipolar couplings. Journal of American Chemical Society. 5, 123, 1014-1015.

Bewley, C. A., Clore, G. M., (2000) Determination of the Relative Orientation of the Two Halves of the Domain-Swapped Dimer of Cyanovirin-N in Solution Using Dipolar Couplings and Rigid Body Minimization. Journal of American Chemical Society. 25, 122, 6009-6016.

Bloembergen, N., (1949) On the Interaction of Nuclear Spins in a Crystalline Lattice. Physica. 3-4, 15, 386-426.

Bradley, P., Misura, K. M., Baker, D., (2005) Toward high-resolution de novo structure prediction for small proteins. Science. 5742, 309, 1868-1871.

Brooks, B. R., Bruccoleri, R. E., Olafson, B. D., States, D. J., Swaminathan, S., Karplus., (1983) CHARMM: A program for macromolecular energy, minimization, and dynamics calculations. Journal of Computational Chemistry. 2, 4, 187-217.

Brunger, A. T., Adams, P. D., Clore, G. M., Delano, W. L., Gros, P., GrosseKunstleve, R. W., Jiang, J. S., Kuszewski, J., Nilges, M., Pannu, N. S., Read, R. J., Rice, L. M., Simonson, T., Warren, G. L., (1998) Crystallography \& NMR system: A new software suite for macromolecular structure determination. Acta. Crystallogr. D. 54, 905-921. 
Brunger, A. T., Adams, P. D., Rice, L. M., (1997) New applications of simulated annealing in X-ray crystallography and solution NMR. Structure. 3, 5, 325336.

Brunger, A. T., Nilges, M., (1993) Computational challenges for macromolecular structure determination by X-ray crystallography and solution NMRspectroscopy. $Q$ Rev Biophys. 1, 26, 49-125.

Brunner, E., (2001) Residual dipolar couplings in protein NMR. Concepts in Magnetic Resonance. 13, 238-259.

Canutescu, A. A., Shelenkov, A. A., Dunbrack, R. L., (2003) A graph-theory algorithm for rapid protein side-chain prediction. Protein Science. 9, 12, 2001-2014.

Castellani, F., Van Rossum, B., Diehl, A., Schubert, M., Rehbein, K., Oschkinat, H., (2002) Structure of a protein determined by solid-state magic-angle-spinning NMR spectroscopy. Nature. 6911, 420, 98-102.

Chapman, D. L., (1913) A contribution to the theory of electrocapillarity. Philosoph. Magaz. 25, 475-481.

Chou, J. J., Bax, A., (2001) Protein side-chain rotamers from dipolar couplings in a liquid crystalline phase. Journal of American Chemical Society. 16, 123, 3844-3845.

Chou, J. J., Delaglio, F., Bax, A., (2000) Measurement of one-bond 15N-13C' dipolar couplings in medium sized proteins. Journal of Biomolecular NMR. 2, 18, 101-105.

Clore, G. M., Garrett, D. S., (1999) R-factor, Free R, and Complete Cross-Validation for Dipolar Coupling Refinement of NMR Structures. Journal of American Chemical Society. 39, 121, 9008-9012.

Clore, G. M., Starich, M. R., Gronenborn, A. M., (1998) Measurement of residual dipolar couplings of macromolecules aligned in the nematic phase of a colloidal suspension of rod-shaped viruses. Journal of American Chemical Society. 40, 120, 10571-10572.

Contreras, M. A., Ubach, J., Millet, O., Rizo, J., Pons, M., (1999) Measurement of one bond dipolar couplings through lanthanide-induced orientation of a calcium-binding protein. Journal of American Chemical Society. 38, 121, 8947-8948.

Cornilescu, G., Delaglio, F., Bax, A., (1999) Protein backbone angle restraints from searching a database for chemical shift and sequence homology. Journal of Biomolecular NMR. 3, 13, 289-302.

Cornilescu, G., Marquardt, J. L., Ottiger, M., Bax, A., (1998) Validation of Protein Structure from Anisotropic Carbonyl Chemical Shifts in a Dilute Liquid 
Crystalline Phase. Journal of American Chemical Society. 27, 120, 68366837.

Davis, M. E., Mccammon, J. A., (1990) Electrostatics in biomolecular structure and dynamics. Chem. Rev. 3, 90, 509-521.

De Alba, E., Baber, J. L., Tjandra, N., (1999) The Use of Residual Dipolar Coupling in Concert with Backbone Relaxation Rates to Identify Conformational Exchange by NMR. Journal of American Chemical Society. 17, 121, 42824283.

De Alba, E., Tjandra, N., (2002) NMR dipolar couplings for the structure determination of biopolymers in solution. Progress in Nuclear Magnetic Resonance Spectroscopy. 2, 40, 175-197.

De La Vega, R. C. R., Merino, E., Becerril, B., Possani, L. D., (2003) Novel interactions between $\mathrm{K}+$ channels and scorpion toxins. Trends in Pharmacological Sciences. 5, 24, 222-227.

Delaglio, F., Grzesiek, S., Vuister, G. W., Zhu, G., Pfeifer, J., Bax, A., (1995) Nmrpipe - a Multidimensional Spectral Processing System Based on Unix Pipes. Journal of Biomolecular NMR. 3, 6, 277-293.

Delaglio, F., Kontaxis, G., Bax, A., (2000) Protein Structure Determination Using Molecular Fragment Replacement and NMR Dipolar Couplings. Journal of American Chemical Society. 9, 122, 2142-2143.

Dingemans, T., Photinos, D. J., Samulski, E. T., Terzis, A. F., Wutz, C., (2003) Ordering of apolar and polar solutes in nematic solvents. Journal of Chemical Physics. 15, 118, 7046-7061.

Dominguez, C., Boelens, R., Bonvin, A. M., (2003) HADDOCK: A protein-protein docking approach based on biochemical or biophysical information. Journal of American Chemical Society. 7, 125, 1731-1737.

Doyle, D. A., Cabral, J. M., Pfuetzner, R. A., Kuo, A. L., Gulbis, J. M., Cohen, S. L., Chait, B. T., Mackinnon, R., (1998) The structure of the potassium channel: Molecular basis of $\mathrm{K}+$ conduction and selectivity. Science. 5360, 280, 69-77.

Eisenhaber, F., Lijnzaad, P., Argos, P., Sander, C., Scharf M., (1995) The double cubic lattice method: Efficient approaches to numerical integration of surface area and volume and to dot surface contouring of molecular assemblies. Journal of Computational Chemistry. 16, 273-284.

Etzkorn, M., Bockmann, A., Lange, A., Baldus, M., (2004) Probing molecular interfaces using 2D magic-angle-spinning NMR on protein mixtures with different uniform labeling. Journal of American Chemical Society. 45, 126, 14746-14751. 
Ferrarini, A., (2003) Modeling of Macromolecular Alignment in Nematic Virus Suspensions. Application to the Prediction of NMR Residual Dipolar Couplings. Journal of Physical Chemistry. B. 31, 107, 7923-7931.

Fischer, M. W., Losonczi, J. A., Weaver, J. L., Prestegard, J. H., (1999) Domain orientation and dynamics in multidomain proteins from residual dipolar couplings. Biochemistry. 28, 38, 9013-9022.

Fleming, K., Gray, D., Prasannan, S., Matthews, S., (2000) Cellulose Crystallites: A New and Robust Liquid Crystalline Medium for the Measurement of Residual Dipolar Couplings. Journal of American Chemical Society. 21, 122, 52245225.

Fossi, M., Castellani, F., Nilges, M., Oschkinat, H., Van Rossum, B. J., (2005) SOLARIA: a protocol for automated cross-peak assignment and structure calculation for solid-state magic-angle spinning NMR spectroscopy. Angewandte Chemie International Edition Engl. 38, 44, 6151-6154.

Franks, W. T., Zhou, D. H., Wylie, B. J., Money, B. G., Graesser, D. T., Frericks, H. L., Sahota, G., Rienstra, C. M., (2005) Magic-angle spinning solid-state NMR spectroscopy of the beta 1 immunoglobulin binding domain of protein $G$ (GB1): N-15 and C-13 chemical shift assignments and conformational analysis. Journal of American Chemical Society. 35, 127, 12291-12305.

Gaemers, S., Bax, A., (2001) Morphology of three lyotropic liquid crystalline biological NMR media studied by translational diffusion anisotropy. Journal of American Chemical Society. 49, 123, 12343-12352.

Gairi, M., Romi, R., Fernandez, I., Rochat, H., Martin-Eauclaire, M. F., Van Rietschoten, J., Pons, M., Giralt, E., (1997) 3D structure of kaliotoxin: is residue 34 a key for channel selectivity? Journal of Peptide Science. 4, 3, 314-319.

Gallagher, K., Sharp, K., (1998) Electrostatic contributions to heat capacity changes of DNA-ligand binding. Biophysical Journal. 2, 75, 769-776.

Gilson, M. K., Sharp, K. A., Honig, B., (1988) Calculating the electrostatic potential of molecules in solution: Method and error assessment. Journal of Computational Chemistry. 4, 9, 327-335.

Glaubitz, C., Watts, A., (1998) Magic angle-oriented sample spinning (MAOSS): A new approach toward biomembrane studies. Journal of Magnetic Resonance. $2, \mathbf{1 3 0}, 305-316$.

Gouaux, E., Mackinnon, R., (2005) Principles of selective ion transport in channels and pumps. Science. 5753, 310, 1461-1465.

Gouy, D. L., (1910) Sur la constitution de la charge electrique a la surface d'un electrolyte. Ann. Phys. 9, 457-468. 
Griffin, R. G., (1998) Dipolar recoupling in MAS spectra of biological solids. Nature Structural Biology. 5 Suppl, 508-512.

Grishaev, A., Bax, A., (2004) An empirical backbone-backbone hydrogen-bonding potential in proteins and its applications to NMR structure refinement and validation. Journal of American Chemical Society. 23, 126, 7281-7292.

Grishaev, A., Llinas, M., (2002a) CLOUDS, a protocol for deriving a molecular proton density via NMR. Proceedings of the National Academy of Sciences $U$ $S$ A. 10, 99, 6707-6712.

Grishaev, A., Llinas, M., (2002b) Protein structure elucidation from NMR proton densities. Proceedings of the National Academy of Sciences U S A. 10, 99, 6713-6718.

Grissmer, S., Nguyen, A. N., Aiyar, J., Hanson, D. C., Mather, R. J., Gutman, G. A., Karmilowicz, M. J., Auperin, D. D., Chandy, K. G., (1994) Pharmacological characterization of five cloned voltage-gated $\mathrm{K}+$ channels, types Kv1.1, 1.2, 1.3, 1.5, and 3.1, stably expressed in mammalian cell lines. Mol Pharmacol. 6, 45, $1227-1234$.

Gronwald, W., Moussa, S., Elsner, R., Jung, A., Ganslmeier, B., Trenner, J., Kremer, W., Neidig, K. P., Kalbitzer, H. R., (2002) Automated assignment of NOESY NMR spectra using a knowledge based method (KNOWNOE). Journal of Biomolecular NMR. 4, 23, 271-287.

Grzesiek, S., Anglister, J., Bax, A., (1993) Correlation of Backbone Amide and Aliphatic Side-Chain Resonances in C-13/N-15-Enriched Proteins by Isotropic Mixing of C-13 Magnetization. Journal of Magnetic Resonance Series B. 1, 101, 114-119.

Grzesiek, S., Bax, A., (1992) Correlating Backbone Amide and Side-Chain Resonances in Larger Proteins by Multiple Relayed Triple Resonance Nmr. Journal of American Chemical Society. 16, 114, 6291-6293.

Guntert, P., (2003) Automated NMR protein structure calculation. Progress in Nuclear Magnetic Resonance Spectroscopy. 3-4, 43, 105-125.

Guntert, P., Mumenthaler, C., Wuthrich, K., (1997) Torsion angle dynamics for NMR structure calculation with the new program DYANA. Journal of Molecular Biology. 1, 273, 283-298.

Hansen, M. R., Mueller, L., Pardi, A., (1998) Tunable alignment of macromolecules by filamentous phage yields dipolar coupling interactions. Nature Structural Biology. 12, 5, 1065-1074.

Heise, H., Seidel, K., Etzkorn, M., Becker, S., Baldus, M., (2005) 3D NMR spectroscopy for resonance assignment and structure elucidation of proteins under MAS: novel pulse schemes and sensitivity considerations. Journal of Magnetic Resonance. 1, 173, 64-74. 
Herrmann, T., Guntert, P., Wuthrich, K., (2002) Protein NMR structure determination with automated NOE assignment using the new software CANDID and the torsion angle dynamics algorithm DYANA. Journal of Molecular Biology. 1, 319, 209-227.

Holst, M., Saied, F., (1993) Multigrid solution of the Poisson-Boltzmann equation. Journal of Computational Chemistry. 1, 14, 105-113.

Honig, B., Nicholls, A., (1995) Classical electrostatics in biology and chemistry. Science. 5214, 268, 1144-1149.

Honig, B., Sharp, K., Yang, A. S., (1993) Macroscopic models of aqueous solutions: biological and chemical applications. Journal of Physical Chemistry. 6, 97, 1101-1109.

Hu, J. S., Bax, A., (1997) chi(1) angle information from a simple two-dimensional NMR experiment that identifies trans (3) J(NC)gamma couplings in isotopically enriched proteins. Journal of Biomolecular NMR. 3, 9, 323-328.

Hu, J. S., Grzesiek, S., Bax, A., (1997) Two-Dimensional NMR Methods for Determining $\chi_{1}$ Angles of Aromatic Residues in Proteins from Three-Bond $\mathrm{J}_{\mathrm{C}^{\prime} \mathrm{\gamma}}$; and $\mathrm{J}_{\mathrm{NC} \gamma}$; Couplings. Journal of American Chemical Society. 7, 119, 1803-1804.

Hubbard, S. J., Thornton, J. M. (Department of Biochemistry and Molecular Biology, University College London, 1993), vol. 54.

Hus, J. C., Marion, D., Blackledge, M., (2001) Determination of protein backbone structure using only residual dipolar couplings. Journal of American Chemical Society. 7, 123, 1541-1542.

Ikura, M., Kay, L. E., Bax, A., (1990) A Novel-Approach for Sequential Assignment of H-1, C-13, and N-15 Spectra of Larger Proteins - Heteronuclear TripleResonance 3-Dimensional Nmr-Spectroscopy - Application to Calmodulin. Biochemistry. 19, 29, 4659-4667.

Ikura, M., Kay, L. E., Tschudin, R., Bax, A., (1990) 3-Dimensional Noesy-Hmqc Spectroscopy of a C-13-Labeled Protein. Journal of Magnetic Resonance. 1, 86, 204-209.

Jaroniec, C. P., Macphee, C. E., Bajaj, V. S., Mcmahon, M. T., Dobson, C. M., Griffin, R. G., (2004) High-resolution molecular structure of a peptide in an amyloid fibril determined by magic angle spinning NMR spectroscopy. Proceedings of the National Academy of Sciences U S A. 3, 101, 711-716.

Jee, J., Guntert, P., (2003) Influence of the completeness of chemical shift assignments on NMR structures obtained with automated NOE assignment. $J$ Struct Funct Genomics. 2-3, 4, 179-189. 
Jeener, J., Meier, B. H., Bachmann, P., Ernst, R. R., (1979) Investigation of Exchange Processes by 2-Dimensional Nmr-Spectroscopy. Journal of Chemical Physics. 11, 71, 4546-4553.

Jiang, Y. X., Lee, A., Chen, J. Y., Cadene, M., Chait, B. T., Mackinnon, R., (2002) The open pore conformation of potassium channels. Nature. $6888,417,523-$ 526.

Jung, Y. S., Sharma, M., Zweckstetter, M., (2004) Simultaneous assignment and structure determination of protein backbones by using NMR dipolar couplings. Angewandte Chemie International Edition Engl. 26, 43, 34793481 .

Jung, Y. S., Zweckstetter, M., (2004a) Backbone assignment of proteins with known structure using residual dipolar couplings. Journal of Biomolecular NMR. 1, 30, 25-35.

Jung, Y. S., Zweckstetter, M., (2004b) Mars -- robust automatic backbone assignment of proteins. Journal of Biomolecular NMR. 1, 30, 11-23.

Kainosho, M., (1997) Isotope labelling of macromolecules for structural determinations. Nature Structural Biology. 4, 858-861.

Kainosho, M., Torizawa, T., Iwashita, Y., Terauchi, T., Ono, A. M., Guntert, P., (2006) Optimal isotope labelling for NMR protein structure determinations. Nature. 7080, 440, 52-57.

Karplus, M., (1963) Vicinal Proton Coupling in Nuclear Magnetic Resonance. Journal of American Chemical Society. 18, 85, 2870.

Kay, L. E., Xu, G. Y., Singer, A. U., Muhandiram, D. R., Formankay, J. D., (1993) A Gradient-Enhanced HCCH-TOCSY Experiment for Recording Side-Chain ${ }^{1} \mathrm{H}$ and ${ }^{13} \mathrm{C}$ Correlations in $\mathrm{H}_{2} \mathrm{O}$ Samples of Proteins. Journal of Magnetic Resonance, Series B. 3, 101, 333-337.

Kern, D., Zuiderweg, E. R. P., (2003) The role of dynamics in allosteric regulation. Current Opinion in Structural Biology. 6, 13, 748-757.

Kirkpatrick, S., Gelatt, C. D., Jr., Vecchi, M. P., (1983) Optimization by Simulated Annealing. Science. 4598, 220, 671-680.

Koenig, B. W., Hu, J. S., Ottiger, M., Bose, S., Hendler, R. W., Bax, A., (1999) NMR measurement of dipolar couplings in proteins aligned by transient binding to purple membrane fragments. Journal of American Chemical Society. 121, 1385-1386.

Korukottu, J., Bayrhuber, M., Montaville, P., Vijayan, V., Jung, Y. S., Becker, S., Zweckstetter, M., (2007) Fast high-resolution protein structure determination by using unassigned NMR data. Angewandte Chemie International Edition Engl. 7, 46, 1176-1179. 
Koshland, D. E., (1958) Application of a Theory of Enzyme Specificity to Protein Synthesis. Proceedings of the National Academy of Sciences USA. 2, 44, 98104.

Kuo, A. L., Gulbis, J. M., Antcliff, J. F., Rahman, T., Lowe, E. D., Zimmer, J., Cuthbertson, J., Ashcroft, F. M., Ezaki, T., Doyle, D. A., (2003) Crystal structure of the potassium channel KirBac1.1 in the closed state. Science. 5627, 300, 1922-1926.

Kuszewski, J., Schwieters, C. D., Garrett, D. S., Byrd, R. A., Tjandra, N., Clore, G. M., (2004) Completely automated, highly error-tolerant macromolecular structure determination from multidimensional nuclear overhauser enhancement spectra and chemical shift assignments. Journal of American Chemical Society. 20, 126, 6258-6273.

Lakomek, N. A., Carlomagno, T., Becker, S., Griesinger, C., Meiler, J., (2006) A thorough dynamic interpretation of residual dipolar couplings in ubiquitin. Journal of Biomolecular NMR. 34, 101-115.

Lange, A., Becker, S., Seidel, K., Giller, K., Pongs, O., Baldus, M., (2005) A concept for rapid protein-structure determination by solid-state NMR spectroscopy. Angewandte Chemie-International Edition. 14, 44, 2089-2092.

Lange, A., Giller, K., Hornig, S., Martin-Eauclaire, M. F., Pongs, O., Becker, S., Baldus, M., (2006) Toxin-induced conformational changes in a potassium channel revealed by solid-state NMR. Nature. 7086, 440, 959-962.

Lange, A., Luca, S., Baldus, M., (2002) Structural constraints from proton-mediated rare-spin correlation spectroscopy in rotating solids. Journal of American Chemical Society. 33, 124, 9704-9705.

Lange, A., Seidel, K., Verdier, L., Luca, S., Baldus, M., (2003) Analysis of protonproton transfer dynamics in rotating solids and their use for 3D structure determination. Journal of American Chemical Society. 41, 125, 1264012648 .

Lee, M., Goldburg, W. I., (1965) Nuclear-magnetic-resonance line narrowing by a rotating rf field. Physical Review. 140, 1261-1271.

Linge, J. P., Habeck, M., Rieping, W., Nilges, M., (2003) ARIA: automated NOE assignment and NMR structure calculation. Bioinformatics. 2, 19, 315-316.

Linge, J. P., O'donoghue, S. I., Nilges, M., (2001) Automated assignment of ambiguous nuclear overhauser effects with ARIA. Methods Enzymol. 339, 71-90.

Linge, J. P., Williams, M. A., Spronk, C. A., Bonvin, A. M., Nilges, M., (2003) Refinement of protein structures in explicit solvent. Proteins. 3, 50, 496-506. 
Liu, G., Shen, Y., Atreya, H. S., Parish, D., Shao, Y., Sukumaran, D. K., Xiao, R., Yee, A., Lemak, A., Bhattacharya, A., Acton, T. A., Arrowsmith, C. H., Montelione, G. T., Szyperski, T., (2005) NMR data collection and analysis protocol for high-throughput protein structure determination. Proceedings of the National Academy of Sciences U S A. 30, 102, 10487-10492.

Long, S. B., Campbell, E. B., Mackinnon, R., (2005) Crystal structure of a mammalian voltage-dependent Shaker family K+ channel. Science. 5736, 309, 897-903.

Luca, S., Heise, H., Baldus, M., (2003) High-resolution solid-state NMR applied to polypeptides and membrane proteins. Accounts of Chemical Research. 11, 36, 858-865.

Macura, S., Ernst, R. R., (1980) Elucidation of Cross Relaxation in Liquids by TwoDimensional Nmr-Spectroscopy. Molecular Physics. 1, 41, 95-117.

Maricq, M. M., Waugh, J. S., (1979) NMR in rotating solids. Journal of Chemical Physics. 7, 70, 3300-3316.

Markus, M. A., Gerstner, R. B., Draper, D. E., Torchia, D. A., (1999) Refining the overall structure and subdomain orientation of ribosomal protein S4 delta41 with dipolar couplings measured by NMR in uniaxial liquid crystalline phases. Journal of Molecular Biology. 2, 292, 375-387.

Marti-Renom, M. A., Stuart, A. C., Fiser, A., Sanchez, R., Melo, F., Sali, A., (2000) Comparative protein structure modeling of genes and genomes. Annual Review of Biophysics and Biomolecular Structure. 29, 291-325.

Mehring, M., Waugh, J. S., (1972) Magic-angle NMR experiments in solids. Physical Review B. 5, 9, 3459-3471.

Meiler, J., (2003) PROSHIFT: protein chemical shift prediction using artificial neural networks. Journal of Biomolecular NMR. 1, 26, 25-37.

Meiler, J., Baker, D., (2003) Rapid protein fold determination using unassigned NMR data. Proceedings of the National Academy of Sciences $U S A .26,100$, 15404-15409.

Meiler, J., Peti, W., Griesinger, C., (2000) DipoCoup: A versatile program for 3Dstructure homology comparison based on residual dipolar couplings and pseudocontact shifts. Journal of Biomolecular NMR, 17, 283-294.

Meiler, J., Prompers, J. J., Peti, W., Griesinger, C., Bruschweiler, R., (2001) ModelFree Approach to the Dynamic Interpretation of Residual Dipolar Couplings in Globular Proteins. Journal of American Chemical Society 25, 123, 60986107.

Miller, C., (1995) The Charybdotoxin Family of K+ Channel-Blocking Peptides. Neuron. 1, 15, 5-10. 
Misra, V. K., Honig, B., (1995) On the magnitude of the electrostatic contribution to ligand-DNA interactions. Proceedings of the National Academy of Sciences $U$ $S$ A. 10, 92, 4691-4695.

Misra, V. K., Sharp, K. A., Friedman, R. A., Honig, B., (1994) Salt effects on ligandDNA binding. Minor groove binding antibiotics. Journal of Molecular Biology. 2, 238, 245-263.

Montelione, G. T., Lyons, B. A., Emerson, S. D., Tashiro, M., (1992) An efficient triple resonance experiment using carbon-13 isotropic mixing for determining sequence-specific resonance assignments of isotopically-enriched proteins. Journal of American Chemical Society. 27, 114, 10974-10975.

Montelione, G. T., Zheng, D., Huang, Y. J., Gunsalus, K. C., Szyperski, T., (2000) Protein NMR spectroscopy in structural genomics. Nature Structural Biology. 7 Suppl, 982-985.

Moseley, H. N., Monleon, D., Montelione, G. T., (2001) Automatic determination of protein backbone resonance assignments from triple resonance nuclear magnetic resonance data. Methods Enzymol. 339, 91-108.

Moseley, H. N., Montelione, G. T., (1999) Automated analysis of NMR assignments and structures for proteins. Current Opinions in Structural Biology. 5, 9, 635642 .

Mumenthaler, C., Braun, W., (1995) Automated assignment of simulated and experimental NOESY spectra of proteins by feedback filtering and selfcorrecting distance geometry. Journal of Molecular Biology. 3, 254, 465480 .

Mumenthaler, C., Guntert, P., Braun, W., Wuthrich, K., (1997) Automated combined assignment of NOESY spectra and three-dimensional protein structure determination. Journal of Biomolecular NMR. 4, 10, 351-362.

Murray, D., Arbuzova, A., Honig, B., Mclaughlin, S., (2002) The role of electrostatic and nonpolar interactions in the association of peripheral proteins with membranes. Peptide-Lipid Interactions. 52, 277-307.

Murray, D., Hermida-Matsumoto, L., Buser, C. A., Tsang, J., Sigal, C. T., Ben-Tal, N., Honig, B., Resh, M. D., Mclaughlin, S., (1998) Electrostatics and the membrane association of Src: theory and experiment. Biochemistry. 8, 37, 2145-2159.

Nederveen, A. J., Doreleijers, J. F., Vranken, W., Miller, Z., Spronk, C. A., Nabuurs, S. B., Guntert, P., Livny, M., Markley, J. L., Nilges, M., Ulrich, E. L., Kaptein, R., Bonvin, A. M., (2005) RECOORD: a recalculated coordinate database of 500+ proteins from the PDB using restraints from the BioMagResBank. Proteins. 4, 59, 662-672. 
Nilges, M., (1993) A calculation strategy for the structure determination of symmetric dimers by 1H NMR. Proteins. 3, 17, 297-309.

Nilges, M., (1995) Calculation of protein structures with ambiguous distance restraints. Automated assignment of ambiguous NOE crosspeaks and disulphide connectivities. Journal of Molecular Biology. 5, 245, 645-660.

Nomura, K., Takegoshi, K., Terao, T., Uchida, K., Kainosho, M., (1999) Determination of the complete structure of a uniformly labeled molecule by rotational resonance solid-state NMR in the tilted rotating frame. Journal of American Chemical Society. 16, 121, 4064-4065.

Osapay, K., Case, D. A., (1994) Analysis of proton chemical shifts in regular secondary structure of proteins. Journal of Biomolecular NMR. 2, 4, 215230 .

Ottiger, M., Delaglio, F., Bax, A., (1998) Measurement of J and dipolar couplings from simplified two-dimensional NMR spectra. Journal of Magnetic Resonance. 2, 131, 373-378.

Peitzsch, R. M., Eisenberg, M., Sharp, K. A., Mclaughlin, S., (1995) Calculations of the electrostatic potential adjacent to model phospholipid bilayers. Biophysical Journal. 3, 68, 729-738.

Permi, P., Annila, A., (2001) A new approach for obtaining sequential assignment of large proteins. Journal of Biomolecular NMR. 20, 127-133.

Peti, W., Meiler, J., Bruschweiler, R., Griesinger, C., (2002) Model-free analysis of protein backbone motion from residual dipolar couplings. Journal of American Chemical Society. 20, 124, 5822-5833.

Petkova, A. T., Ishii, Y., Balbach, J. J., Antzutkin, O. N., Leapman, R. D., Delaglio, F., Tycko, R., (2002) A structural model for Alzheimer's beta -amyloid fibrils based on experimental constraints from solid state NMR. Proceedings of the National Academy of Sciences U S A. 26, 99, 16742-16747.

Photinos, D. J., Poon, C. D., Samulski, E. T., Toriumi, H., (1992) Nmr-Study of the Effects of Electric-Dipole Interactions on the Ordering of Chain Solutes in the Nematic Phase. Journal of Physical Chemistry. 20, 96, 8176-8180.

Photinos, D. J., Samulski, E. T., (1993) Electric-Dipole Interactions of Chain Molecules in Nematics - the Analysis of Segmental Ordering in Alpha, Omega-Dibromoalkanes. Journal of Chemical Physics. 12, 98, 10009-10016.

Pickford, A. R., Campbell, I. D., (2004) NMR studies of modular protein structures and their interactions. Chemical Reviews. 8, 104, 3557-3565.

Prestegard, J. H., (1998) New techniques in structural NMR - anisotropic interactions. Nature Structural Biology. 5, 517-522. 
Prestegard, J. H., Al-Hashimi, H. M., Tolman, J. R., (2000) NMR structures of biomolecules using field oriented media and residual dipolar couplings. $Q R e v$ Biophys. 4, 33, 371-424.

Prestegard, J. H., Kishore, A. I., (2001) Partial alignment of biomolecules: an aid to NMR characterization. Curr Opin Chem Biol. 5, 5, 584-590.

Ramirez, B. E., Bax, A., (1998) Modulation of the Alignment Tensor of Macromolecules Dissolved in a Dilute Liquid Crystalline Medium. Journal of American Chemical Society. 35, 120, 9106-9107.

Ramirez, B. E., Voloshin, O. N., Camerini-Otero, R. D., Bax, A., (2000) Solution structure of DinI provides insight into its mode of RecA inactivation. Protein Sci. 11, 9, 2161-2169.

Ranganathan, R., Lewis, J. H., Mackinnon, R., (1996) Spatial localization of the K+ channel selectivity filter by mutant cycle-based structure analysis. Neuron. 1, 16, 131-139.

Rienstra, C. M., Tucker-Kellogg, L., Jaroniec, C. P., Hohwy, M., Reif, B., Mcmahon, M. T., Tidor, B., Lozano-Perez, T., Griffin, R. G., (2002) De novo determination of peptide structure with solid-state magic-angle spinning NMR spectroscopy. Proceedings of the National Academy of Sciences $U S A$. 16, 99, 10260-10265.

Rohl, C. A., Baker, D., (2002) De novo determination of protein backbone structure from residual dipolar couplings using Rosetta. Journal of American Chemical Society. 11, 124, 2723-2729.

Ruckert, M., Otting, G., (2000) Alignment of Biological Macromolecules in Novel Nonionic Liquid Crystalline Media for NMR Experiments. Journal of American Chemical Society 32, 122, 7793-7797.

Sanders, C. R., Schaff, J. E., Prestegard, J. H., (1993) Orientational behavior of phosphatidylcholine bilayers in the presence of aromatic amphiphiles and a magnetic field. Biophysical Journal. 64,1069-1080.

Saupe, A., Englert, G., (1963) High-Resolution Nuclear Magnetic Resonance Spectra of Orientated Molecules. Physical Review Letters. 10, 11, 462.

Schwieters, C. D., Kuszewski, J. J., Tjandra, N., Clore, G. M., (2003) The Xplor-NIH NMR molecular structure determination package. Journal of Magnetic Resonance. 1, 160, 65-73.

Seidel, K., Lange, A., Becker, S., Hughes, C. E., Heise, H., Baldus, M., (2004) Protein solid-state NMR resonance assignments from (C-13, C-13) correlation spectroscopy. Physical Chemistry Chemical Physics. 22, 6, 5090-5093. 
Sharp, K. A., Honig, B., (1990) Calculating total electrostatic energies with the nonlinear Poisson-Boltzmann equation. Journal of Physical Chemistry. 19, 94, 7684-7692.

Sharp, K. A., Honig, B., (1990) Electrostatic interactions in macromolecules: theory and applications. Annu Rev Biophys Biophys Chem. 19, 301-332.

Shen, Y., Atreya, H. S., Liu, G. H., Szyperski, T., (2005) G-matrix Fourier transform NOESY-based protocol for high-quality protein structure determination. Journal of American Chemical Society. 25, 127, 9085-9099.

Skrynnikov, N. R., Goto, N. K., Yang, D., Choy, W. Y., Tolman, J. R., Mueller, G. A., Kay, L. E., (2000) Orienting domains in proteins using dipolar couplings measured by liquid-state NMR: differences in solution and crystal forms of maltodextrin binding protein loaded with beta-cyclodextrin. Journal of Molecular Biology. 5, 295, 1265-1273.

Solomon, I., (1955) Relaxation Processes in a System of 2 Spins. Physical Review. 2, 99, 559-565.

Stein, E. G., Rice, L. M., Brunger, A. T., (1997) Torsion-angle molecular dynamics as a new efficient tool for NMR structure calculation. Journal of Magnetic Resonance. 1, 124, 154-164.

Suter, D., Ernst, R. R., (1985) Spin Diffusion in Resolved Solid-State Nmr-Spectra. Physical Review B. 9, 32, 5608-5627.

Syvitski, R. T., Burnell, E. E., (1997) Dipole-induced ordering in nematic liquid crystals fact or fiction? Chemical Physics Letters. 1-3, 281, 199-206.

Syvitski, R. T., Burnell, E. E., (2000) Dipole-induced ordering in nematic liquid crystals. II. The elusive holy grail. Journal of Chemical Physics. 8, 113, 3452-3465.

Takegoshi, K., Nakamura, S., Terao, T., (2001) C-13-H-1 dipolar-assisted rotational resonance in magic-angle spinning NMR. Chemical Physics Letters. 5-6, 344, 631-637.

Terzis, A. F., Poon, C. D., Samulski, E. T., Luz, Z., Poupko, R., Zimmermann, H., Muller, K., Toriumi, H., Photinos, D. J., (1996) Shape-Dominated Ordering in Nematic Solvents. A Deuterium NMR Study of Cycloalkane Solutes. Journal of American Chemical Society. 9, 118, 2226-2234.

Thompson, J. D., Higgins, D. G., Gibson, T. J., (1994) Clustal-W - Improving the Sensitivity of Progressive Multiple Sequence Alignment through Sequence Weighting, Position-Specific Gap Penalties and Weight Matrix Choice. Nucleic Acids Research. 22, 22, 4673-4680. 
Tjandra, N., Bax, A., (1997) Direct measurement of distances and angles in biomolecules by NMR in a dilute liquid crystalline medium. Science. 5340, 278, 1111-1114.

Tjandra, N., Omichinski, J. G., Gronenborn, A. M., Clore, G. M., Bax, A., (1997) Use of dipolar $1 \mathrm{H}-15 \mathrm{~N}$ and $1 \mathrm{H}-13 \mathrm{C}$ couplings in the structure determination of magnetically oriented macromolecules in solution. Nature Structural Biology. $9,4,732-738$.

Tolman, J. R., (2002) A novel approach to the retrieval of structural and dynamic information from residual dipolar couplings using several oriented media in biomolecular NMR spectroscopy. Journal of American Chemical Society. 40, 124, $12020-12030$.

Tolman, J. R., Flanagan, J. M., Kennedy, M. A., Prestegard, J. H., (1995) Nuclear Magnetic Dipole Interactions in Field-Oriented Proteins - Information for Structure Determination in Solution. Proceedings of the National Academy of Sciences U S A. 20, 92, 9279-9283.

Tycko, R., (1996) Prospects for resonance assignments in multidimensional solidstate NMR spectra of uniformly labeled proteins. Journal of Biomolecular NMR. 3, 8, 239-251.

Tycko, R., (2001) Biomolecular solid state NMR: Advances in structural methodology and applications to peptide and protein fibrils. Annual Review of Physical Chemistry. 52, 575-606.

Valafar, H., Prestegard, J. H., (2003) Rapid classification of a protein fold family using a statistical analysis of dipolar couplings. Bioinformatics. 12, 19, 15491555 .

Van Rossum, B. J., Castellani, F., Pauli, J., Rehbein, K., Hollander, J., De Groot, H. J. M., Oschkinat, H., (2003) Assignment of amide proton signals by combined evaluation of HN, NN and HNCA MAS-NMR correlation spectra. Journal of Biomolecular NMR. 3, 25, 217-223.

Vijayan, V., Zweckstetter, M., (2005) Simultaneous measurement of protein one-bond residual dipolar couplings without increased resonance overlap. Journal of Magnetic Resonance. 2, 174, 245-253.

Warren, J. J., Moore, P. B., (2001) Application of dipolar coupling data to the refinement of the solution structure of the sarcin-ricin loop RNA. Journal of Biomolecular NMR. 4, 20, 311-323.

Wishart, D. S., Sykes, B. D., (1994) Chemical shifts as a tool for structure determination in Nuclear Magnetic Resonance, Pt C, Volume 239, Methods in Enzymology. 363-392.

Wittekind, M., Mueller, L., (1993) Hncacb, a High-Sensitivity 3d NMR Experiment to Correlate Amide-Proton and Nitrogen Resonances with the Alpha-Carbon 
and Beta-Carbon Resonances in Proteins. Journal of Magnetic Resonance Series B. 2, 101, 201-205.

Woolf, T. B., Roux, B., (1996) Structure, energetics, and dynamics of lipid-protein interactions: a molecular dynamics study of the gramicidin A channel in a DMPC bilaer. Proteins. 24, 92-114.

Wuthrich, K., (1986) NMR of proteins and nucleic acids. Wliey, New york.

Wuthrich, K., (2003) NMR studies of structure and function of biological macromolecules (Nobel lecture). Angew Chem Int Ed Engl. 29, 42, 33403363.

Wuthrich, K., Wider, G., Wagner, G., Braun, W., (1982) Sequential resonance assignments as a basis for determination of spatial protein structures by highresolution proton nuclear magnetic resonance. Journal of Molecular Biology. $3, \mathbf{1 5 5}, 311-319$.

Yamazaki, T., Forman-Kay, J. D., Kay, L. E., (1993) Two-dimensional NMR experiments for correlating carbon-13.beta. and proton.delta./.epsilon. chemical shifts of aromatic residues in 13C-labeled proteins via scalar couplings. Journal of American Chemical Society. 23, 115, 11054-11055.

Zech, S. G., Wand, A. J., Mcdermott, A. E., (2005) Protein structure determination by high-resolution solid-state NMR spectroscopy: application to microcrystalline ubiquitin. Journal of American Chemical Society. 24, 127, 8618-8626.

Zhou, F., Schulten, K., (1995) Molecular dynamics study of a membrane-water interface. Journal of Physical Chemistry. 99, 2195-2207.

Zhou, Y. F., Morais-Cabral, J. H., Kaufman, A., Mackinnon, R., (2001) Chemistry of ion coordination and hydration revealed by a $\mathrm{K}+$ channel-Fab complex at 2.0 angstrom resolution. Nature. 6859, 414, 43-48.

Zweckstetter, M., Bax, A., (2000) Prediction of Sterically Induced Alignment in a Dilute Liquid Crystalline Phase: Aid to Protein Structure Determination by NMR. Journal of American Chemical Society. 15, 122, 3791-3792.

Zweckstetter, M., Bax, A., (2001) Characterization of molecular alignment in aqueous suspensions of Pf1 bacteriophage. Journal of Biomolecular NMR. 4, 20, 365377.

Zweckstetter, M., Hummer, G., Bax, A., (2004) Prediction of charge-induced molecular alignment of biomolecules dissolved in dilute liquid-crystalline phases. Biophys J. 6, 86, 3444-3460. 


\section{Appendix A}

\section{Chemical shift assignment of KTX in solution-state}

\begin{tabular}{|c|c|c|c|c|c|c|}
\hline 1 & 43.502 & $\mathrm{CA}$ & 1 & 45 & 1.689 & HB3 \\
\hline 2 & 3.988 & QA & 1 & 46 & 1.53 & QD \\
\hline 3 & 61.434 & $\mathrm{CA}$ & 2 & 47 & 1.448 & QG \\
\hline 4 & 34.274 & $\mathrm{CB}$ & 2 & 48 & 8.446 & $\mathrm{H}$ \\
\hline 5 & 4.303 & HA & 2 & 49 & 125.793 & $\mathrm{~N}$ \\
\hline 6 & 1.91 & $\mathrm{HB}$ & 2 & 50 & 53.855 & $\mathrm{CA}$ \\
\hline 7 & 0.882 & QQG & 2 & 51 & 47.115 & $\mathrm{CB}$ \\
\hline 8 & 8.846 & $\mathrm{H}$ & 2 & 52 & 4.87 & HA \\
\hline 9 & 117.577 & $\mathrm{~N}$ & 2 & 53 & 3.778 & HB3 \\
\hline 10 & 55.85 & CA & 3 & 54 & 3.162 & HB2 \\
\hline 11 & 34.069 & $\mathrm{CB}$ & 3 & 55 & 2.854 & $\mathrm{HG}$ \\
\hline 12 & 4.296 & HA & 3 & 56 & 7.935 & $\mathrm{H}$ \\
\hline 13 & 2.077 & HB2 & 3 & 57 & 114.088 & $\mathrm{~N}$ \\
\hline 14 & 1.865 & HB3 & 3 & 58 & 57.997 & $\mathrm{CA}$ \\
\hline 15 & 1.537 & QG & 3 & 59 & 64.589 & $\mathrm{CB}$ \\
\hline 16 & 8.693 & $\mathrm{H}$ & 3 & 61 & 4.619 & $\mathrm{HA}$ \\
\hline 17 & 125.316 & $\mathrm{~N}$ & 3 & 62 & 3.997 & HB2 \\
\hline 18 & 59.821 & CA & 4 & 63 & 3.784 & HB3 \\
\hline 19 & 40.435 & $\mathrm{CB}$ & 4 & 64 & 9.342 & $\mathrm{H}$ \\
\hline 20 & 4.584 & HA & 4 & 65 & 112.099 & $\mathrm{~N}$ \\
\hline 21 & 1.865 & $\mathrm{HB}$ & 4 & 66 & 44.364 & $\mathrm{CA}$ \\
\hline 22 & 0.942 & QG1 & 4 & 68 & 4.444 & QA \\
\hline 23 & 0.748 & QG2 & 4 & 69 & 7.696 & $\mathrm{H}$ \\
\hline 24 & 8.24 & $\mathrm{H}$ & 4 & 70 & 108.998 & $\mathrm{~N}$ \\
\hline 25 & 118.472 & $\mathrm{~N}$ & 4 & 71 & 61.458 & $\mathrm{CA}$ \\
\hline 26 & 52.797 & $\mathrm{CA}$ & 5 & 72 & 61.323 & CB \\
\hline 27 & 36.966 & $\mathrm{CB}$ & 5 & 73 & 4.191 & HA \\
\hline 28 & 4.805 & HA & 5 & 74 & 4.118 & HB3 \\
\hline 29 & 2.912 & HB2 & 5 & 75 & 4.12 & HB2 \\
\hline 30 & 2.596 & HB3 & 5 & 76 & 9.385 & $\mathrm{H}$ \\
\hline 31 & 8.699 & $\mathrm{H}$ & 5 & 77 & 118.787 & $\mathrm{~N}$ \\
\hline 32 & 119.879 & $\mathrm{~N}$ & 5 & 78 & 57.427 & $\mathrm{CA}$ \\
\hline 33 & 61.748 & $\mathrm{CA}$ & 6 & 79 & 29.223 & $\mathrm{CB}$ \\
\hline 34 & 36.251 & $\mathrm{CB}$ & 6 & 80 & 34.321 & CG \\
\hline 35 & 4.134 & HA & 6 & 81 & 4.194 & HA \\
\hline 36 & 1.386 & HB & 6 & 82 & 2.227 & QB \\
\hline 37 & 0.931 & QG1 & 6 & 83 & 2.551 & $\mathrm{HG} 2$ \\
\hline 38 & 0.851 & QG2 & 6 & 84 & 2.386 & HG3 \\
\hline 39 & 8.045 & $\mathrm{H}$ & 6 & 85 & 7.312 & $\mathrm{H}$ \\
\hline 40 & 121.024 & $\mathrm{~N}$ & 6 & 86 & 111.535 & $\mathrm{~N}$ \\
\hline 41 & 56.061 & CA & 7 & 87 & 4.582 & HA \\
\hline 42 & 33.283 & $\mathrm{CB}$ & 7 & 88 & 3.096 & QB \\
\hline 43 & 4.826 & HA & 7 & 89 & 2.568 & $\mathrm{HG}$ \\
\hline 44 & 1.769 & HB2 & 7 & 90 & 8.256 & $\mathrm{H}$ \\
\hline
\end{tabular}




\begin{tabular}{|c|c|c|c|c|c|c|c|}
\hline 91 & 111.534 & $\mathrm{~N}$ & 14 & 143 & 9.068 & $\mathrm{H}$ & 20 \\
\hline 92 & 59.041 & $\mathrm{CA}$ & 15 & 144 & 120.47 & $\mathrm{~N}$ & 20 \\
\hline 93 & 41.45 & $\mathrm{CB}$ & 15 & 145 & 51.697 & $\mathrm{CA}$ & 21 \\
\hline 94 & 4.095 & HA & 15 & 146 & 18.65 & $\mathrm{CB}$ & 21 \\
\hline 95 & 1.813 & HB2 & 15 & 147 & 4.49 & HA & 21 \\
\hline 96 & 1.691 & HB3 & 15 & 148 & 1.489 & $\mathrm{HB}$ & 21 \\
\hline 97 & 1.005 & HD1 & 15 & 149 & 7.355 & $\mathrm{H}$ & 21 \\
\hline 98 & 0.882 & HD2 & 15 & 150 & 119.295 & $\mathrm{~N}$ & 21 \\
\hline 99 & 7.19 & $\mathrm{H}$ & 15 & 151 & 45.701 & $\mathrm{CA}$ & 22 \\
\hline 100 & 122.213 & $\mathrm{~N}$ & 15 & 153 & 3.845 & QA & 22 \\
\hline 101 & 60.766 & CA & 16 & 154 & 8.028 & $\mathrm{H}$ & 22 \\
\hline 102 & 29.49 & $\mathrm{CB}$ & 16 & 155 & 105.76 & $\mathrm{~N}$ & 22 \\
\hline 103 & 4.22 & HA & 16 & 156 & 54.312 & $\mathrm{CA}$ & 23 \\
\hline 104 & 1.727 & HB2 & 16 & 157 & 33.31 & $\mathrm{CB}$ & 23 \\
\hline 105 & 1.651 & HB3 & 16 & 158 & 4.607 & HA & 23 \\
\hline 106 & 1.94 & HE2 & 16 & 159 & 2.052 & HB2 & 23 \\
\hline 107 & 1.824 & HE3 & 16 & 160 & 1.755 & HB3 & 23 \\
\hline 108 & 1.457 & HG2 & 16 & 161 & 2.413 & HG2 & 23 \\
\hline 109 & 1.37 & HG3 & 16 & 162 & 2.317 & HG3 & 23 \\
\hline 110 & 9.028 & $\mathrm{H}$ & 16 & 163 & 8.099 & $\mathrm{H}$ & 23 \\
\hline 111 & 118.647 & $\mathrm{~N}$ & 16 & 164 & 117.91 & $\mathrm{~N}$ & 23 \\
\hline 113 & 66.436 & CA & 17 & 165 & 57.604 & CA & 24 \\
\hline 114 & 31.432 & $\mathrm{CB}$ & 17 & 166 & 38.402 & $\mathrm{CB}$ & 24 \\
\hline 115 & 28.368 & $\mathrm{CG}$ & 17 & 167 & 4.425 & HA & 24 \\
\hline 116 & 4.284 & HA & 17 & 168 & 1.354 & HB2 & 24 \\
\hline 117 & 2.334 & HB2 & 17 & 169 & 1.269 & HB3 & 24 \\
\hline 118 & 2.094 & HB3 & 17 & 170 & 2.847 & HD2 & 24 \\
\hline 119 & 3.647 & HD2 & 17 & 171 & 2.8 & HD3 & 24 \\
\hline 120 & 3.537 & HD3 & 17 & 172 & 1.15 & HG2 & 24 \\
\hline 121 & 1.658 & QG & 17 & 173 & 1.048 & HG3 & 24 \\
\hline 122 & 36.325 & $\mathrm{CB}$ & 18 & 174 & 8.374 & $\mathrm{H}$ & 24 \\
\hline 123 & 4.424 & HA & 18 & 175 & 115.225 & $\mathrm{~N}$ & 24 \\
\hline 124 & 3.034 & QB & 18 & 176 & 56.882 & $\mathrm{CA}$ & 25 \\
\hline 125 & 2.382 & $\mathrm{HG}$ & 18 & 177 & 38.009 & $\mathrm{CB}$ & 25 \\
\hline 126 & 7.719 & $\mathrm{H}$ & 18 & 178 & 4.459 & HA & 25 \\
\hline 127 & 112.583 & $\mathrm{~N}$ & 18 & 179 & 3.255 & HB2 & 25 \\
\hline 128 & 59.608 & CA & 19 & 180 & 3.163 & HB3 & 25 \\
\hline 129 & 32.139 & $\mathrm{CB}$ & 19 & 181 & 8.177 & $\mathrm{H}$ & 25 \\
\hline 130 & 4.283 & HA & 19 & 182 & 113.537 & $\mathrm{~N}$ & 25 \\
\hline 131 & 1.966 & HB2 & 19 & 183 & 46.159 & $\mathrm{CA}$ & 26 \\
\hline 132 & 1.915 & HB3 & 19 & 184 & 4.726 & HA2 & 26 \\
\hline 133 & 1.668 & HD2 & 19 & 185 & 3.782 & HA3 & 26 \\
\hline 134 & 1.55 & HD3 & 19 & 186 & 7.935 & $\mathrm{H}$ & 26 \\
\hline 135 & 1.428 & QG & 19 & 187 & 106.22 & $\mathrm{~N}$ & 26 \\
\hline 136 & 8.057 & $\mathrm{H}$ & 19 & 188 & 35.151 & $\mathrm{CB}$ & 27 \\
\hline 137 & 122.728 & $\mathrm{~N}$ & 19 & 189 & 4.634 & HA & 27 \\
\hline 138 & 56.049 & CA & 20 & 190 & 1.688 & HB2 & 27 \\
\hline 139 & 40.472 & $\mathrm{CB}$ & 20 & 191 & 1.615 & HB3 & 27 \\
\hline 140 & 4.414 & HA & 20 & 192 & 1.352 & HG2 & 27 \\
\hline 141 & 2.822 & HB3 & 20 & 193 & 1.265 & HG3 & 27 \\
\hline 142 & 2.829 & HB2 & 20 & 194 & 8.873 & $\mathrm{H}$ & 27 \\
\hline
\end{tabular}




\begin{tabular}{|c|c|c|c|c|c|c|}
\hline 195 & 121.393 & $\mathrm{~N}$ & 27 & 233 & 5.364 & HA \\
\hline 196 & 55.519 & CA & 28 & 234 & 1.79 & QB \\
\hline 197 & 38.209 & $\mathrm{CB}$ & 28 & 235 & 1.586 & QD \\
\hline 198 & 4.884 & HA & 28 & 236 & 1.939 & QE \\
\hline 199 & 2.823 & HB2 & 28 & 237 & 1.445 & QG \\
\hline 200 & 2.495 & HB3 & 28 & 238 & 7.761 & $\mathrm{H}$ \\
\hline 201 & 8.865 & $\mathrm{H}$ & 28 & 239 & 117.452 & $\mathrm{~N}$ \\
\hline 202 & 125.044 & $\mathrm{~N}$ & 28 & 240 & 55.451 & CA \\
\hline 203 & 54.868 & CA & 29 & 241 & 38.209 & $\mathrm{CB}$ \\
\hline 204 & 36.879 & $\mathrm{CB}$ & 29 & 242 & 5.064 & HA \\
\hline 205 & 31.115 & $\mathrm{CG}$ & 29 & 243 & 3.947 & HB2 \\
\hline 207 & 4.657 & HA & 29 & 244 & 2.665 & HB3 \\
\hline 208 & 2.141 & HB3 & 29 & 245 & 2.503 & HG \\
\hline 209 & 1.691 & HB2 & 29 & 246 & 8.434 & $\mathrm{H}$ \\
\hline 210 & 1.33 & $\mathrm{QE}$ & 29 & 247 & 122.075 & $\mathrm{~N}$ \\
\hline 211 & 2.247 & $\mathrm{HG} 2$ & 29 & 248 & 52.271 & CA \\
\hline 212 & 2.127 & HG3 & 29 & 249 & 30.939 & $\mathrm{CB}$ \\
\hline 213 & 8.716 & $\mathrm{H}$ & 29 & 250 & 5.332 & HA \\
\hline 214 & 127.77 & $\mathrm{~N}$ & 29 & 251 & 3.154 & HB2 \\
\hline 215 & 54.606 & CA & 30 & 252 & 3.023 & HB3 \\
\hline 216 & 37.296 & $\mathrm{CB}$ & 30 & 253 & 9.502 & $\mathrm{H}$ \\
\hline 217 & 4.331 & HA & 30 & 254 & 119.271 & $\mathrm{~N}$ \\
\hline 218 & 3.023 & HB2 & 30 & 255 & 54.196 & CA \\
\hline 219 & 2.748 & HB3 & 30 & 256 & 37.546 & CB \\
\hline 220 & 9.485 & $\mathrm{H}$ & 30 & 257 & 5.329 & HA \\
\hline 221 & 124.232 & $\mathrm{~N}$ & 30 & 258 & 3.097 & HB3 \\
\hline 222 & 58.079 & CA & 31 & 259 & 3.095 & HB2 \\
\hline 223 & 4.035 & HA & 31 & 260 & 2.676 & $\mathrm{HG}$ \\
\hline 224 & 2.264 & HB2 & 31 & 261 & 9.245 & $\mathrm{H}$ \\
\hline 225 & 2.172 & HB3 & 31 & 262 & 119.717 & $\mathrm{~N}$ \\
\hline 226 & 3.255 & HD2 & 31 & 263 & 60.073 & CA \\
\hline 227 & 3.2 & HD3 & 31 & 264 & 71.108 & $\mathrm{CB}$ \\
\hline 228 & 1.595 & QG & 31 & 265 & 4.941 & HA \\
\hline 229 & 8.6 & $\mathrm{H}$ & 31 & 266 & 4.004 & $\mathrm{HB}$ \\
\hline 230 & 105.574 & $\mathrm{~N}$ & 31 & 267 & 1.402 & $\mathrm{HG} 2$ \\
\hline 231 & 54.653 & CA & 32 & 268 & 8.766 & $\mathrm{H}$ \\
\hline 232 & 36.702 & $\mathrm{CB}$ & 32 & 269 & 117.142 & $\mathrm{~N}$ \\
\hline
\end{tabular}




\section{Appendix B}

Distance restraints of KTX in solid-state (free form)

\begin{tabular}{|c|c|c|c|c|c|c|c|c|c|c|c|}
\hline 12 & PRO & HA & 15 & LEU & HG & 32 & LYS & $\mathrm{HA}$ & 32 & LYS & $Q D$ \\
\hline 9 & SER & QB & 10 & GLY & $\mathrm{QA}$ & 4 & ILE & QG1 & 35 & CYS & $\mathrm{HA}$ \\
\hline 4 & ILE & HB & 6 & VAL & HA & 3 & GLU & $\mathrm{QB}$ & 4 & ILE & QG1 \\
\hline 6 & VAL & HA & 7 & LYS & HA & 4 & ILE & QG1 & 33 & CYS & QB \\
\hline 36 & THR & HA & 37 & PRO & $\mathrm{QD}$ & 12 & PRO & $\mathrm{QB}$ & 16 & LYS & QG \\
\hline 1 & GLY & QA & 2 & VAL & HA & 15 & LEU & QD1 & 25 & PHE & $\mathrm{HA}$ \\
\hline 15 & LEU & HA & 15 & LEU & QB & 29 & MET & $\mathrm{QB}$ & 32 & LYS & QG \\
\hline 18 & CYS & HA & 21 & ALA & QB & 4 & ILE & QD1 & 6 & VAL & QG1 \\
\hline 13 & GLN & HA & 16 & LYS & QB & 6 & VAL & QG2 & 7 & LYS & QD \\
\hline 6 & VAL & HA & 7 & LYS & HA & 18 & CYS & $\mathrm{HA}$ & 21 & ALA & $\mathrm{QB}$ \\
\hline 7 & LYS & QG & 32 & LYS & HA & 18 & CYS & $\mathrm{HA}$ & 21 & $A L A$ & $\mathrm{QB}$ \\
\hline 11 & SER & HA & 28 & CYS & HA & 21 & ALA & QB & 35 & CYS & $\mathrm{QB}$ \\
\hline 23 & MET & $\mathrm{HA}$ & 37 & PRO & QB & 4 & ILE & QG2 & 6 & VAL & $\mathrm{HB}$ \\
\hline 8 & CYS & HA & 17 & PRO & QD & 4 & ILE & QD1 & 17 & PRO & QG \\
\hline 5 & ASN & HA & 5 & ASN & QB & 4 & ILE & QD1 & 17 & PRO & $\mathrm{QB}$ \\
\hline 33 & CYS & HA & 34 & HIS & HA & 4 & ILE & QD1 & 6 & VAL & QG1 \\
\hline 36 & THR & HA & 37 & PRO & QD & 4 & ILE & QD1 & 21 & ALA & $\mathrm{QB}$ \\
\hline 35 & CYS & $\mathrm{HA}$ & 37 & PRO & QD & 2 & VAL & HB & 34 & HIS & $\mathrm{HA}$ \\
\hline 16 & LYS & HA & 17 & PRO & QD & 12 & PRO & $\mathrm{HA}$ & 15 & LEU & HG \\
\hline 8 & CYS & HA & 17 & PRO & QD & 12 & PRO & $\mathrm{QB}$ & 13 & GLN & QG \\
\hline 12 & PRO & QB & 17 & PRO & QD & 15 & LEU & $\mathrm{HA}$ & 25 & PHE & $\mathrm{HA}$ \\
\hline 8 & CYS & QB & 10 & GLY & QA & 3 & GLU & $\mathrm{HA}$ & 34 & HIS & $\mathrm{HA}$ \\
\hline 1 & GLY & $\mathrm{QA}$ & 3 & GLU & HA & 16 & LYS & $\mathrm{HA}$ & 17 & PRO & QG \\
\hline 1 & GLY & QA & 2 & VAL & HA & 6 & VAL & $\mathrm{HA}$ & 6 & VAL & QG1 \\
\hline 15 & LEU & QB & 16 & LYS & QG & 3 & GLU & $\mathrm{HA}$ & 33 & CYS & $\mathrm{QB}$ \\
\hline 15 & LEU & HA & 15 & LEU & QB & 3 & GLU & QG & 32 & LYS & QG \\
\hline 4 & ILE & HB & 33 & CYS & QB & 2 & VAL & QG1 & 3 & GLU & QG \\
\hline 33 & CYS & $\mathrm{QB}$ & 34 & HIS & HA & 32 & LYS & QG & 32 & LYS & QD \\
\hline 4 & ILE & QG1 & 33 & CYS & QB & 7 & LYS & QG & 32 & LYS & $Q B$ \\
\hline 10 & GLY & $\mathrm{QA}$ & 28 & CYS & QB & 3 & GLU & $\mathrm{HA}$ & 4 & ILE & $\mathrm{HA}$ \\
\hline 33 & CYS & HA & 33 & CYS & QB & 2 & VAL & $\mathrm{HA}$ & 35 & CYS & $\mathrm{HA}$ \\
\hline 6 & VAL & HB & 7 & LYS & QB & 8 & CYS & $\mathrm{HA}$ & 9 & SER & $\mathrm{QB}$ \\
\hline 6 & VAL & HB & 17 & PRO & QB & 8 & CYS & $\mathrm{HA}$ & 9 & SER & $\mathrm{HA}$ \\
\hline 4 & ILE & HA & 6 & VAL & HB & 8 & CYS & QB & 33 & CYS & $\mathrm{HA}$ \\
\hline 29 & $\mathrm{MET}$ & QB & 29 & MET & QG & 2 & VAL & $\mathrm{HA}$ & 2 & VAL & $\mathrm{HB}$ \\
\hline 2 & VAL & HB & 2 & VAL & QG1 & 3 & GLU & QG & 4 & ILE & $\mathrm{HA}$ \\
\hline 3 & GLU & QB & 32 & LYS & QB & 12 & PRO & QB & 13 & GLN & $\mathrm{HA}$ \\
\hline 2 & VAL & HB & 37 & PRO & QB & 32 & LYS & QG & 33 & CYS & $Q B$ \\
\hline 29 & MET & QB & 29 & MET & QG & 32 & LYS & $\mathrm{QB}$ & 32 & LYS & $\mathrm{QD}$ \\
\hline 16 & LYS & QG & 17 & PRO & QB & 8 & CYS & QB & 13 & GLN & $\mathrm{QB}$ \\
\hline 6 & VAL & HB & 17 & PRO & QB & 7 & LYS & $\mathrm{HA}$ & 32 & LYS & QG \\
\hline 23 & $\mathrm{MET}$ & QB & 37 & PRO & QD & 10 & GLY & $\mathrm{QA}$ & 11 & SER & $\mathrm{HA}$ \\
\hline 7 & LYS & QD & 32 & LYS & HA & 23 & MET & $\mathrm{QB}$ & 36 & THR & $\mathrm{HA}$ \\
\hline 27 & LYS & QG & 27 & LYS & QD & 4 & ILE & $\mathrm{HA}$ & 32 & LYS & QG \\
\hline 3 & GLU & HA & 32 & LYS & QD & 3 & GLU & $\mathrm{HA}$ & 4 & ILE & QG1 \\
\hline 6 & VAL & HB & 17 & PRO & QG & 12 & PRO & $\mathrm{HA}$ & 13 & GLN & QG \\
\hline
\end{tabular}




\begin{tabular}{|c|c|c|c|c|c|c|c|c|c|c|c|}
\hline 36 & THR & HB & 36 & THR & QG2 & 17 & PRO & QB & 17 & PRO & $\mathrm{QD}$ \\
\hline 12 & PRO & $\mathrm{HA}$ & 12 & PRO & $\mathrm{QB}$ & 27 & LYS & $\mathrm{QB}$ & 27 & LYS & $\mathrm{QD}$ \\
\hline 12 & PRO & $\mathrm{HA}$ & 12 & PRO & QG & 3 & GLU & QB & 32 & LYS & $\mathrm{QD}$ \\
\hline 9 & SER & $\mathrm{HA}$ & 9 & SER & $\mathrm{QB}$ & 16 & LYS & $\mathrm{QB}$ & 16 & LYS & QG \\
\hline 11 & SER & $\mathrm{HA}$ & 11 & SER & $\mathrm{QB}$ & 37 & PRO & QG & 37 & PRO & QB \\
\hline 10 & GLY & $\mathrm{QA}$ & 11 & SER & $\mathrm{QB}$ & 15 & LEU & HG & 25 & PHE & HA \\
\hline 37 & PRO & $\mathrm{HA}$ & 37 & PRO & $\mathrm{QB}$ & 4 & ILE & QG1 & 33 & CYS & $\mathrm{HA}$ \\
\hline 35 & CYS & $\mathrm{QB}$ & 37 & PRO & $\mathrm{HA}$ & 4 & ILE & QG1 & 4 & ILE & QD1 \\
\hline 6 & VAL & HA & 6 & VAL & $\mathrm{HB}$ & 4 & ILE & QG1 & 32 & LYS & HA \\
\hline 6 & VAL & $\mathrm{HA}$ & 6 & VAL & QG2 & 7 & LYS & $\mathrm{HA}$ & 7 & LYS & QG \\
\hline 2 & VAL & $\mathrm{HA}$ & 37 & PRO & $\mathrm{QD}$ & 6 & VAL & QG2 & 17 & PRO & QG \\
\hline 16 & LYS & $\mathrm{HA}$ & 16 & LYS & $\mathrm{QB}$ & 6 & VAL & QG2 & 17 & PRO & QB \\
\hline 16 & LYS & HA & 16 & LYS & QG & 4 & ILE & QG2 & 5 & ASN & $\mathrm{QB}$ \\
\hline 4 & ILE & $\mathrm{HA}$ & 4 & ILE & QG1 & 4 & ILE & QG2 & 4 & ILE & $\mathrm{HB}$ \\
\hline 4 & ILE & HA & 4 & ILE & $\mathrm{HB}$ & 4 & ILE & QG2 & 4 & ILE & QD1 \\
\hline 13 & GLN & HA & 13 & GLN & $Q B$ & 4 & ILE & $\mathrm{HA}$ & 4 & ILE & QG2 \\
\hline 3 & GLU & $\mathrm{HA}$ & 3 & GLU & $\mathrm{QB}$ & 4 & ILE & QG2 & 4 & ILE & QG1 \\
\hline 7 & LYS & $\mathrm{HA}$ & 7 & LYS & QB & 4 & ILE & QD1 & 33 & CYS & $\mathrm{HA}$ \\
\hline 32 & LYS & $\mathrm{HA}$ & 32 & LYS & QG & 4 & ILE & $\mathrm{HB}$ & 4 & ILE & QD1 \\
\hline 29 & MET & HA & 29 & MET & $\mathrm{QB}$ & 7 & LYS & HA & 7 & LYS & QD \\
\hline 28 & CYS & HA & 28 & CYS & $\mathrm{QB}$ & 37 & PRO & QG & 37 & PRO & $\mathrm{QD}$ \\
\hline 35 & CYS & $\mathrm{HA}$ & 35 & CYS & $\mathrm{QB}$ & 3 & GLU & $\mathrm{QB}$ & 3 & GLU & QG \\
\hline 23 & MET & $\mathrm{HA}$ & 23 & MET & $\mathrm{QB}$ & 11 & SER & $\mathrm{HA}$ & 12 & PRO & QG \\
\hline 8 & CYS & $\mathrm{HA}$ & 8 & CYS & $\mathrm{QB}$ & 15 & LEU & $\mathrm{HA}$ & 15 & LEU & HG \\
\hline 24 & ARG & $\mathrm{HA}$ & 24 & ARG & QB & 17 & PRO & $\mathrm{HA}$ & 18 & CYS & HA \\
\hline 4 & ILE & $\mathrm{HA}$ & 5 & ASN & $\mathrm{HA}$ & 11 & SER & $\mathrm{HA}$ & 28 & CYS & HA \\
\hline 11 & SER & HA & 12 & PRO & QD & 17 & PRO & $Q D$ & 18 & CYS & HA \\
\hline 12 & PRO & $Q D$ & 12 & PRO & $\mathrm{QB}$ & 8 & CYS & $\mathrm{QB}$ & 9 & SER & HA \\
\hline 12 & PRO & $\mathrm{QD}$ & 12 & PRO & QG & 8 & CYS & $\mathrm{QB}$ & 13 & GLN & HA \\
\hline 21 & $A L A$ & $\mathrm{HA}$ & 21 & ALA & $\mathrm{QB}$ & 1 & GLY & $\mathrm{QA}$ & 4 & ILE & HA \\
\hline 12 & PRO & $\mathrm{QD}$ & 12 & PRO & $\mathrm{HA}$ & 11 & SER & $\mathrm{HA}$ & 15 & LEU & $\mathrm{QB}$ \\
\hline 17 & PRO & QD & 17 & PRO & QG & 37 & PRO & QB & 37 & PRO & QD \\
\hline 16 & LYS & QB & 17 & PRO & QD & 2 & VAL & QG2 & 3 & GLU & $\mathrm{HA}$ \\
\hline 10 & GLY & $\mathrm{QA}$ & 12 & PRO & QD & 6 & VAL & QG1 & 32 & LYS & HA \\
\hline 1 & GLY & $\mathrm{QA}$ & 37 & PRO & $Q D$ & 21 & ALA & QB & 35 & CYS & $\mathrm{QB}$ \\
\hline 15 & LEU & $\mathrm{QB}$ & 15 & LEU & HG & 2 & VAL & QG2 & 35 & CYS & QB \\
\hline 4 & ILE & HB & 4 & ILE & QG1 & 37 & PRO & QD & 37 & PRO & $\mathrm{QB}$ \\
\hline 25 & PHE & HA & 25 & PHE & QB & 37 & PRO & $Q D$ & 37 & PRO & QB \\
\hline 4 & ILE & QG1 & 6 & VAL & HB & 4 & ILE & HA & 5 & ASN & QB \\
\hline 32 & LYS & HA & 32 & LYS & QB & 37 & PRO & $\mathrm{HA}$ & 37 & PRO & QG \\
\hline 4 & ILE & $\mathrm{HB}$ & 6 & VAL & $\mathrm{HB}$ & 2 & VAL & $\mathrm{HA}$ & 37 & PRO & QG \\
\hline 6 & VAL & $\mathrm{HB}$ & 6 & VAL & QG1 & 25 & PHE & $\mathrm{QB}$ & 27 & LYS & $\mathrm{QD}$ \\
\hline 6 & VAL & $\mathrm{HB}$ & 6 & VAL & QG2 & 21 & ALA & QB & 35 & CYS & QB \\
\hline 29 & MET & QG & 32 & LYS & $\mathrm{QB}$ & 15 & LEU & QD2 & 15 & LEU & HG \\
\hline 32 & LYS & QB & 32 & LYS & QG & 2 & VAL & QG2 & 37 & PRO & QG \\
\hline 4 & ILE & QD1 & 6 & VAL & HB & 26 & GLY & $\mathrm{QA}$ & 35 & CYS & $\mathrm{QB}$ \\
\hline 2 & VAL & HB & 2 & VAL & QG2 & 34 & HIS & $\mathrm{HA}$ & 35 & CYS & QB \\
\hline 13 & GLN & $\mathrm{HA}$ & 13 & GLN & QG & 36 & THR & $\mathrm{HA}$ & 37 & PRO & $\mathrm{QD}$ \\
\hline 7 & LYS & QB & 7 & LYS & $Q D$ & 35 & CYS & $\mathrm{QB}$ & 36 & THR & HB \\
\hline 7 & LYS & $\mathrm{QB}$ & 7 & LYS & QG & 17 & PRO & $\mathrm{HA}$ & 17 & PRO & QG \\
\hline 2 & VAL & $\mathrm{HA}$ & 37 & PRO & QB & 17 & PRO & $\mathrm{HA}$ & 17 & PRO & $\mathrm{QB}$ \\
\hline 29 & MET & $\mathrm{HA}$ & 29 & MET & QG & 17 & PRO & $\mathrm{HA}$ & 21 & $A L A$ & HA \\
\hline 12 & PRO & $\mathrm{QB}$ & 12 & PRO & QG & 12 & PRO & $\mathrm{HA}$ & 15 & LEU & HG \\
\hline
\end{tabular}




$\begin{array}{rllrllllllll}9 & \text { SER } & \text { QB } & 10 & \text { GLY } & \text { QA } & 2 & \text { VAL } & \text { HB } & 37 & \text { PRO } & \text { QB } \\ 15 & \text { LEU } & \text { QD1 } & 16 & \text { LYS } & \text { HA } & 23 & \text { MET } & \text { QB } & 37 & \text { PRO } & \text { QD } \\ 29 & \text { MET } & \text { QB } & 33 & \text { CYS } & \text { HA } & 13 & \text { GLN } & \text { QB } & 13 & \text { GLN } & \text { QG } \\ 28 & \text { CYS } & \text { HA } & 33 & \text { CYS } & \text { HA } & 32 & \text { LYS } & \text { HA } & 32 & \text { LYS } & \text { QD } \\ 12 & \text { PRO } & \text { QB } & 17 & \text { PRO } & \text { QD } & 6 & \text { VAL } & \text { HB } & 17 & \text { PRO } & \text { QG } \\ 15 & \text { LEU } & \text { QB } & 25 & \text { PHE } & \text { QB } & 16 & \text { LYS } & \text { QG } & 17 & \text { PRO } & \text { QB } \\ 15 & \text { LEU } & \text { HA } & 15 & \text { LEU } & \text { QB } & 15 & \text { LEU } & \text { QD1 } & 15 & \text { LEU } & \text { HG } \\ 11 & \text { SER } & \text { QB } & 15 & \text { LEU } & \text { QB } & 4 & \text { ILE } & \text { QD1 } & 6 & \text { VAL } & \text { QG1 } \\ 5 & \text { ASN } & \text { HA } & 5 & \text { ASN } & \text { QB } & 6 & \text { VAL } & \text { QG1 } & 17 & \text { PRO } & \text { QB } \\ 3 & \text { GLU } & \text { QB } & 32 & \text { LYS } & \text { QB } & 4 & \text { ILE } & \text { QD1 } & 5 & \text { ASN } & \text { QB } \\ 3 & \text { GLU } & \text { QB } & 32 & \text { LYS } & \text { QG } & 4 & \text { ILE } & \text { QD1 } & 17 & \text { PRO } & \text { QB } \\ 36 & \text { THR } & \text { HA } & 37 & \text { PRO } & \text { QB } & & & & & & \end{array}$




\section{Appendix C}

\section{Chemical shift assignment of Conk-S2}

\begin{tabular}{|c|c|c|c|c|c|c|c|}
\hline 1 & 1.390 & HB & 1 & 47 & 1.896 & HB2 & 6 \\
\hline 2 & 3.688 & HA & 1 & 48 & 3.148 & HD2 & 6 \\
\hline 3 & 7.876 & $\mathrm{H}$ & 1 & 49 & 3.175 & HD3 & 6 \\
\hline 4 & 1.526 & HG3 & 2 & 50 & 4.513 & $\mathrm{HA}$ & 6 \\
\hline 5 & 1.640 & HG2 & 2 & 51 & 8.280 & $\mathrm{H}$ & 6 \\
\hline 6 & 1.978 & HB3 & 2 & 52 & 53.840 & $C A$ & 6 \\
\hline 7 & 1.998 & HB2 & 2 & 53 & 122.000 & $\mathrm{~N}$ & 6 \\
\hline 8 & 3.164 & HD2 & 2 & 54 & 1.951 & HB3 & 7 \\
\hline 9 & 3.193 & HD3 & 2 & 55 & 1.979 & HG2 & 7 \\
\hline 10 & 4.714 & $\mathrm{HA}$ & 2 & 56 & 2.022 & HG 3 & 7 \\
\hline 11 & 8.200 & $\mathrm{H}$ & 2 & 57 & 2.352 & HB2 & 7 \\
\hline 12 & 54.280 & $\mathrm{CA}$ & 2 & 58 & 3.807 & HD3 & 7 \\
\hline 13 & 118.900 & $\mathrm{~N}$ & 2 & 59 & 3.825 & HD2 & 7 \\
\hline 14 & 2.017 & HB3 & 3 & 60 & 4.534 & $\mathrm{HA}$ & 7 \\
\hline 15 & 2.051 & HG2 & 3 & 61 & 3.959 & HB2 & 8 \\
\hline 16 & 2.102 & HG3 & 3 & 62 & 3.989 & HB3 & 8 \\
\hline 17 & 2.309 & HB2 & 3 & 63 & 4.345 & $\mathrm{HA}$ & 8 \\
\hline 18 & 4.087 & HD3 & 3 & 64 & 8.136 & $\mathrm{H}$ & 8 \\
\hline 19 & 4.185 & HD2 & 3 & 65 & 3.048 & HB3 & 9 \\
\hline 20 & 4.449 & $\mathrm{HA}$ & 3 & 66 & 3.081 & HB2 & 9 \\
\hline 21 & 1.394 & HG3 & 4 & 67 & 4.470 & $\mathrm{HA}$ & 9 \\
\hline 22 & 1.394 & HG2 & 4 & 68 & 6.824 & $\mathrm{HE} 2$ & 9 \\
\hline 23 & 1.720 & HB3 & 4 & 69 & 6.980 & $\mathrm{H}$ & 9 \\
\hline 24 & 1.720 & HD2 & 4 & 70 & 7.200 & HD2 & 9 \\
\hline 25 & 1.720 & HD3 & 4 & 71 & 36.420 & $\mathrm{CB}$ & 9 \\
\hline 26 & 1.720 & HB2 & 4 & 72 & 57.840 & $\mathrm{CA}$ & 9 \\
\hline 27 & 2.964 & $\mathrm{HE} 3$ & 4 & 73 & 116.300 & $\mathrm{~N}$ & 9 \\
\hline 28 & 2.964 & $\mathrm{HE} 2$ & 4 & 74 & 2.671 & QG & 10 \\
\hline 29 & 4.230 & $\mathrm{HA}$ & 4 & 75 & 2.808 & HB2 & 10 \\
\hline 30 & 8.270 & $\mathrm{H}$ & 4 & 76 & 2.808 & HB3 & 10 \\
\hline 31 & 24.710 & CG & 4 & 77 & 4.387 & $\mathrm{HA}$ & 10 \\
\hline 32 & 29.230 & $C D$ & 4 & 78 & 7.410 & $\mathrm{H}$ & 10 \\
\hline 33 & 42.160 & $\mathrm{CE}$ & 4 & 79 & 38.640 & $\mathrm{CB}$ & 10 \\
\hline 34 & 56.260 & $\mathrm{CA}$ & 4 & 80 & 57.760 & $\mathrm{CA}$ & 10 \\
\hline 35 & 33.240 & $\mathrm{CB}$ & 4 & 81 & 119.500 & $\mathrm{~N}$ & 10 \\
\hline 36 & 121.200 & $\mathrm{~N}$ & 4 & 82 & 2.700 & HB3 & 11 \\
\hline 37 & 2.460 & HB2 & 5 & 83 & 3.050 & HB2 & 11 \\
\hline 38 & 2.573 & HB3 & 5 & 84 & 4.950 & $\mathrm{HA}$ & 11 \\
\hline 39 & 4.556 & HA & 5 & 85 & 7.400 & $\mathrm{H}$ & 11 \\
\hline 40 & 8.040 & $\mathrm{H}$ & 5 & 86 & 52.860 & $\mathrm{CA}$ & 11 \\
\hline 41 & 41.860 & $\mathrm{CB}$ & 5 & 87 & 39.510 & $\mathrm{CB}$ & 11 \\
\hline 42 & 53.820 & $\mathrm{CA}$ & 5 & 88 & 44.160 & CG & 11 \\
\hline 43 & 120.400 & $\mathrm{~N}$ & 5 & 89 & 111.000 & $\mathrm{~N}$ & 11 \\
\hline 44 & 1.384 & HG3 & 6 & 90 & 0.825 & HD2 & 12 \\
\hline 45 & 1.431 & HG2 & 6 & 91 & 1.557 & HB2 & 12 \\
\hline 46 & 1.640 & HB3 & 6 & 92 & 1.671 & HG & 12 \\
\hline
\end{tabular}




\begin{tabular}{|c|c|c|c|c|c|c|c|}
\hline 93 & 1.718 & HB3 & 12 & 146 & 8.080 & $\mathrm{H}$ & 20 \\
\hline 94 & 4.292 & HA & 12 & 147 & 21.420 & $\mathrm{CG} 2$ & 20 \\
\hline 95 & 7.510 & $\mathrm{H}$ & 12 & 148 & 69.230 & $\mathrm{CB}$ & 20 \\
\hline 96 & 53.250 & $C A$ & 12 & 149 & 60.350 & $\mathrm{CA}$ & 20 \\
\hline 97 & 121.800 & $\mathrm{~N}$ & 12 & 150 & 106.000 & $\mathrm{~N}$ & 20 \\
\hline 98 & 1.973 & HB3 & 13 & 151 & 1.266 & HG3 & 21 \\
\hline 99 & 2.006 & HG 2 & 13 & 152 & 1.448 & HG2 & 21 \\
\hline 100 & 2.056 & HG3 & 13 & 153 & 1.684 & HD3 & 21 \\
\hline 101 & 2.288 & HB2 & 13 & 154 & 1.732 & HD2 & 21 \\
\hline 102 & 3.840 & HD2 & 13 & 155 & 1.837 & HB3 & 21 \\
\hline 103 & 3.961 & HD3 & 13 & 156 & 2.193 & HB2 & 21 \\
\hline 104 & 4.271 & $\mathrm{HA}$ & 13 & 157 & 2.920 & $\mathrm{HE} 3$ & 21 \\
\hline 105 & 0.561 & HB & 14 & 158 & 2.975 & $\mathrm{HE} 2$ & 21 \\
\hline 106 & 3.422 & HA & 14 & 159 & 4.478 & $\mathrm{HA}$ & 21 \\
\hline 107 & 7.040 & $\mathrm{H}$ & 14 & 160 & 7.740 & $\mathrm{H}$ & 21 \\
\hline 108 & 51.960 & $\mathrm{CA}$ & 14 & 161 & 53.070 & $\mathrm{CA}$ & 21 \\
\hline 109 & 15.360 & $\mathrm{CB}$ & 14 & 162 & 122.900 & $\mathrm{~N}$ & 21 \\
\hline 110 & 120.700 & $\mathrm{~N}$ & 14 & 163 & 1.914 & HB3 & 22 \\
\hline 111 & 2.609 & HB2 & 15 & 164 & 1.940 & HG3 & 22 \\
\hline 112 & 2.303 & HB3 & 15 & 165 & 1.982 & HG2 & 22 \\
\hline 113 & 4.844 & $\mathrm{HA}$ & 15 & 166 & 2.198 & HB2 & 22 \\
\hline 114 & 7.640 & $\mathrm{H}$ & 15 & 167 & 3.436 & HD3 & 22 \\
\hline 115 & 52.070 & $\mathrm{CA}$ & 15 & 168 & 3.844 & HD2 & 22 \\
\hline 116 & 43.630 & $\mathrm{CB}$ & 15 & 169 & 4.082 & $\mathrm{HA}$ & 22 \\
\hline 117 & 39.780 & CG & 15 & 170 & 2.116 & HB2 & 23 \\
\hline 118 & 121.600 & $\mathrm{~N}$ & 15 & 171 & 2.288 & HG3 & 23 \\
\hline 119 & 4.680 & $\mathrm{HA}$ & 16 & 172 & 2.288 & HG2 & 23 \\
\hline 120 & 4.083 & HB2 & 16 & 173 & 2.116 & HB3 & 23 \\
\hline 121 & 3.941 & HB3 & 16 & 174 & 4.606 & $\mathrm{HA}$ & 23 \\
\hline 122 & 9.080 & $\mathrm{H}$ & 16 & 175 & 9.070 & $\mathrm{H}$ & 23 \\
\hline 123 & 64.300 & $\mathrm{CB}$ & 16 & 176 & 41.340 & $C D$ & 23 \\
\hline 124 & 61.770 & $\mathrm{CA}$ & 16 & 177 & 36.340 & CG & 23 \\
\hline 125 & 122.100 & $\mathrm{~N}$ & 16 & 178 & 34.440 & $\mathrm{CB}$ & 23 \\
\hline 126 & 3.763 & HA2 & 17 & 179 & 54.590 & $\mathrm{CA}$ & 23 \\
\hline 127 & 4.268 & HA3 & 17 & 180 & 120.900 & $\mathrm{~N}$ & 23 \\
\hline 128 & 8.890 & $\mathrm{H}$ & 17 & 181 & 1.993 & HB2 & 24 \\
\hline 129 & 45.510 & $\mathrm{CA}$ & 17 & 182 & 1.993 & HB3 & 24 \\
\hline 130 & 108.400 & $\mathrm{~N}$ & 17 & 183 & 2.195 & HG2 & 24 \\
\hline 131 & 3.695 & HB2 & 18 & 184 & 2.334 & HG3 & 24 \\
\hline 132 & 3.695 & HB3 & 18 & 185 & 4.658 & $\mathrm{HA}$ & 24 \\
\hline 133 & 4.773 & $\mathrm{HA}$ & 18 & 186 & 8.670 & $\mathrm{H}$ & 24 \\
\hline 134 & 8.740 & $\mathrm{H}$ & 18 & 187 & 29.940 & $\mathrm{CB}$ & 24 \\
\hline 135 & 65.610 & $\mathrm{CB}$ & 18 & 188 & 48.280 & $C D$ & 24 \\
\hline 136 & 56.630 & $\mathrm{CA}$ & 18 & 189 & 56.240 & $\mathrm{CA}$ & 24 \\
\hline 137 & 117.900 & $\mathrm{~N}$ & 18 & 190 & 121.800 & $\mathrm{~N}$ & 24 \\
\hline 138 & 3.617 & HA 3 & 19 & 191 & 1.802 & HB2 & 25 \\
\hline 139 & 4.598 & $\mathrm{HA} 2$ & 19 & 192 & 1.240 & HG2 & 25 \\
\hline 140 & 7.920 & $\mathrm{H}$ & 19 & 193 & 1.802 & HB3 & 25 \\
\hline 141 & 43.350 & $\mathrm{CA}$ & 19 & 194 & 1.187 & HG 3 & 25 \\
\hline 142 & 105.300 & $\mathrm{~N}$ & 19 & 195 & 3.307 & HD2 & 25 \\
\hline 143 & 1.029 & HG 2 & 20 & 196 & 2.817 & HD3 & 25 \\
\hline 144 & 4.544 & $\mathrm{HA}$ & 20 & 197 & 4.654 & HA & 25 \\
\hline 145 & 4.544 & HB & 20 & 198 & 8.720 & $\mathrm{H}$ & 25 \\
\hline
\end{tabular}




\begin{tabular}{|c|c|c|c|c|c|c|}
\hline 199 & 34.600 & $\mathrm{CB}$ & 25 & 252 & 7.920 & $\mathrm{H}$ \\
\hline 200 & 29.140 & CG & 25 & 253 & 18.400 & $\mathrm{CB}$ \\
\hline 201 & 42.610 & $C D$ & 25 & 254 & 54.700 & $\mathrm{CA}$ \\
\hline 202 & 52.550 & $\mathrm{CA}$ & 25 & 255 & 124.900 & $\mathrm{~N}$ \\
\hline 203 & 127.500 & $\mathrm{~N}$ & 25 & 256 & 1.158 & HG3 \\
\hline 204 & 0.941 & HG2 & 26 & 257 & 1.296 & HG2 \\
\hline 205 & 1.267 & HG 12 & 26 & 258 & 1.741 & HB2 \\
\hline 206 & 1.140 & HG 13 & 26 & 259 & 1.741 & HB3 \\
\hline 207 & 0.755 & QD1 & 26 & 260 & 1.488 & HD2 \\
\hline 208 & 1.700 & HB & 26 & 261 & 1.488 & HD3 \\
\hline 209 & 5.315 & $\mathrm{HA}$ & 26 & 262 & 2.890 & HE3 \\
\hline 210 & 8.880 & $\mathrm{H}$ & 26 & 263 & 2.890 & $\mathrm{HE} 2$ \\
\hline 211 & 28.010 & CG1 & 26 & 264 & 4.088 & HA \\
\hline 212 & 20.780 & CG2 & 26 & 265 & 7.540 & $\mathrm{H}$ \\
\hline 213 & 12.170 & $\mathrm{CD} 1$ & 26 & 266 & 41.970 & $\mathrm{CE}$ \\
\hline 214 & 40.640 & $\mathrm{CB}$ & 26 & 267 & 29.100 & $C D$ \\
\hline 215 & 58.630 & $\mathrm{CA}$ & 26 & 268 & 25.290 & CG \\
\hline 216 & 119.700 & $\mathrm{~N}$ & 26 & 269 & 33.760 & CB \\
\hline 217 & 2.715 & HB2 & 27 & 270 & 55.880 & $\mathrm{CA}$ \\
\hline 218 & 2.715 & HB3 & 27 & 271 & 115.200 & $\mathrm{~N}$ \\
\hline 219 & 5.220 & $\mathrm{HA}$ & 27 & 272 & 1.256 & HG 3 \\
\hline 220 & 6.638 & $\mathrm{HE} 2$ & 27 & 273 & 1.301 & HG2 \\
\hline 221 & 7.165 & HD2 & 27 & 274 & 1.652 & HD2 \\
\hline 222 & 9.870 & $\mathrm{H}$ & 27 & 275 & 1.652 & HD3 \\
\hline 223 & 54.810 & $\mathrm{CA}$ & 27 & 276 & 1.704 & HB3 \\
\hline 224 & 41.420 & $\mathrm{CB}$ & 27 & 277 & 1.855 & HB2 \\
\hline 225 & 126.500 & $\mathrm{~N}$ & 27 & 278 & 2.998 & $\mathrm{HE} 3$ \\
\hline 226 & 2.696 & HB2 & 28 & 279 & 2.056 & $\mathrm{HE} 2$ \\
\hline 227 & 3.428 & HB3 & 28 & 280 & 3.544 & HA \\
\hline 228 & 4.304 & $\mathrm{HA}$ & 28 & 281 & 7.870 & $\mathrm{H}$ \\
\hline 229 & 6.674 & $\mathrm{HE} 2$ & 28 & 282 & 24.940 & $C D$ \\
\hline 230 & 6.878 & HD2 & 28 & 283 & 29.090 & $\mathrm{CB}$ \\
\hline 231 & 10.370 & $\mathrm{H}$ & 28 & 284 & 42.610 & $\mathrm{CE}$ \\
\hline 232 & 58.130 & $\mathrm{CA}$ & 28 & 285 & 56.940 & $\mathrm{CA}$ \\
\hline 233 & 39.080 & $\mathrm{CB}$ & 28 & 286 & 116.400 & $\mathrm{~N}$ \\
\hline 234 & 123.200 & $\mathrm{~N}$ & 28 & 287 & 1.375 & HB2 \\
\hline 235 & 1.912 & HB3 & 29 & 288 & 1.375 & HB3 \\
\hline 236 & 2.992 & HB2 & 29 & 289 & 2.173 & HG2 \\
\hline 237 & 4.395 & $\mathrm{HA}$ & 29 & 290 & 1.828 & HG3 \\
\hline 238 & 8.030 & $\mathrm{H}$ & 29 & 291 & 4.520 & HA \\
\hline 239 & 44.180 & CG & 29 & 292 & 6.780 & $\mathrm{H}$ \\
\hline 240 & 39.050 & $\mathrm{CB}$ & 29 & 293 & 34.430 & $\mathrm{CB}$ \\
\hline 241 & 51.680 & $C A$ & 29 & 294 & 33.160 & CG \\
\hline 242 & 126.600 & $\mathrm{~N}$ & 29 & 295 & 50.140 & $C D$ \\
\hline 243 & 2.824 & HB2 & 30 & 296 & 53.810 & $\mathrm{CA}$ \\
\hline 244 & 3.058 & HB3 & 30 & 297 & 112.300 & $\mathrm{~N}$ \\
\hline 245 & 4.023 & $\mathrm{HA}$ & 30 & 298 & 2.643 & HG \\
\hline 246 & 8.310 & $\mathrm{H}$ & 30 & 299 & 3.171 & HB2 \\
\hline 247 & 63.250 & $\mathrm{CB}$ & 30 & 300 & 3.171 & HB3 \\
\hline 248 & 61.100 & $\mathrm{CA}$ & 30 & 301 & 5.223 & $\mathrm{HA}$ \\
\hline 249 & 121.400 & $\mathrm{~N}$ & 30 & 302 & 8.500 & $\mathrm{H}$ \\
\hline 250 & 1.455 & HB & 31 & 303 & 44.600 & $\mathrm{CB}$ \\
\hline 251 & 4.199 & HA & 31 & 304 & 57.780 & $\mathrm{CA}$ \\
\hline
\end{tabular}




\begin{tabular}{|c|c|c|c|c|c|c|c|}
\hline 305 & 122.500 & $\mathrm{~N}$ & 35 & 358 & 4.470 & HA 3 & 42 \\
\hline 306 & 2.127 & HB & 36 & 359 & 7.237 & $\mathrm{H}$ & 42 \\
\hline 307 & 4.659 & HA & 36 & 360 & 1.246 & HG 3 & 43 \\
\hline 308 & 9.170 & $\mathrm{H}$ & 36 & 361 & 1.502 & HD2 & 43 \\
\hline 309 & 0.880 & HG 1 & 36 & 362 & 1.502 & HD3 & 43 \\
\hline 310 & 0.599 & HG2 & 36 & 363 & 1.246 & HG2 & 43 \\
\hline 311 & 22.230 & CG1 & 36 & 364 & 1.582 & HB3 & 43 \\
\hline 312 & 20.190 & CG2 & 36 & 365 & 1.582 & HB2 & 43 \\
\hline 313 & 35.760 & $\mathrm{CB}$ & 36 & 366 & 2.878 & HE 3 & 43 \\
\hline 314 & 60.210 & $\mathrm{CA}$ & 36 & 367 & 2.878 & $\mathrm{HE} 2$ & 43 \\
\hline 315 & 122.700 & $\mathrm{~N}$ & 36 & 368 & 4.643 & $\mathrm{HA}$ & 43 \\
\hline 316 & 1.079 & HG2 & 37 & 369 & 8.140 & $\mathrm{H}$ & 43 \\
\hline 317 & 4.209 & $\mathrm{HB}$ & 37 & 370 & 24.730 & CG & 43 \\
\hline 318 & 5.456 & HA & 37 & 371 & 29.390 & $C D$ & 43 \\
\hline 319 & 7.970 & $\mathrm{H}$ & 37 & 372 & 35.640 & $\mathrm{CB}$ & 43 \\
\hline 320 & 22.180 & CG2 & 37 & 373 & 42.280 & $\mathrm{CE}$ & 43 \\
\hline 321 & 71.250 & $\mathrm{CB}$ & 37 & 374 & 55.020 & $\mathrm{CA}$ & 43 \\
\hline 322 & 61.990 & $\mathrm{CA}$ & 37 & 375 & 121.500 & $\mathrm{~N}$ & 43 \\
\hline 323 & 112.800 & $\mathrm{~N}$ & 37 & 376 & 3.775 & HA2 & 44 \\
\hline 324 & 2.961 & HB3 & 38 & 377 & 4.128 & HA 3 & 44 \\
\hline 325 & 3.216 & HB2 & 38 & 378 & 8.590 & $\mathrm{H}$ & 44 \\
\hline 326 & 4.942 & $\mathrm{HA}$ & 38 & 379 & 45.170 & $\mathrm{CA}$ & 44 \\
\hline 327 & 6.527 & $\mathrm{HE} 2$ & 38 & 380 & 106.500 & $\mathrm{~N}$ & 44 \\
\hline 328 & 7.091 & HD2 & 38 & 381 & 3.676 & HA 3 & 45 \\
\hline 329 & 9.460 & $\mathrm{H}$ & 38 & 382 & 4.532 & HA2 & 45 \\
\hline 330 & 41.190 & $\mathrm{CB}$ & 38 & 383 & 9.200 & $\mathrm{H}$ & 45 \\
\hline 331 & 56.070 & $\mathrm{CA}$ & 38 & 384 & 43.700 & $\mathrm{CA}$ & 45 \\
\hline 332 & 121.300 & $\mathrm{~N}$ & 38 & 385 & 112.800 & $\mathrm{~N}$ & 45 \\
\hline 333 & 0.958 & HG 1 & 39 & 386 & 2.893 & HB2 & 46 \\
\hline 334 & 1.810 & HG2 & 39 & 387 & 2.968 & HB3 & 46 \\
\hline 335 & 4.289 & HA & 39 & 388 & 4.870 & HA & 46 \\
\hline 336 & 3.919 & HB & 39 & 389 & 9.050 & $\mathrm{H}$ & 46 \\
\hline 337 & 8.540 & $\mathrm{H}$ & 39 & 390 & 40.750 & CB & 46 \\
\hline 338 & 22.420 & CG2 & 39 & 391 & 51.530 & CA & 46 \\
\hline 339 & 62.730 & $\mathrm{CA}$ & 39 & 392 & 118.800 & $\mathrm{~N}$ & 46 \\
\hline 340 & 69.060 & $\mathrm{CB}$ & 39 & 393 & 3.578 & HA2 & 47 \\
\hline 341 & 117.000 & $\mathrm{~N}$ & 39 & 394 & 4.210 & HA 3 & 47 \\
\hline 342 & 2.454 & HB2 & 40 & 395 & 8.270 & $\mathrm{H}$ & 47 \\
\hline 343 & 2.226 & HB3 & 40 & 396 & 45.530 & $\mathrm{CA}$ & 47 \\
\hline 344 & 4.804 & $\mathrm{HA}$ & 40 & 397 & 104.000 & $\mathrm{~N}$ & 47 \\
\hline 345 & 6.858 & $\mathrm{HE} 2$ & 40 & 398 & 1.912 & HB3 & 48 \\
\hline 346 & 6.868 & HD2 & 40 & 399 & 3.213 & HB2 & 48 \\
\hline 347 & 8.860 & $\mathrm{H}$ & 40 & 400 & 4.691 & $\mathrm{HA}$ & 48 \\
\hline 348 & 41.880 & $\mathrm{CB}$ & 40 & 401 & 8.180 & $\mathrm{H}$ & 48 \\
\hline 349 & 54.580 & $\mathrm{CA}$ & 40 & 402 & 34.980 & $\mathrm{CB}$ & 48 \\
\hline 350 & 129.100 & $\mathrm{~N}$ & 40 & 403 & 50.410 & $\mathrm{CA}$ & 48 \\
\hline 351 & 2.572 & HB3 & 41 & 404 & 120.600 & $\mathrm{~N}$ & 48 \\
\hline 352 & 2.713 & HB2 & 41 & 405 & 2.372 & HB2 & 49 \\
\hline 353 & 4.455 & HA & 41 & 406 & 2.460 & HB3 & 49 \\
\hline 354 & 9.140 & $\mathrm{H}$ & 41 & 407 & 4.715 & HA & 49 \\
\hline 355 & 55.630 & $\mathrm{CA}$ & 41 & 408 & 6.650 & $\mathrm{H}$ & 49 \\
\hline 356 & 124.300 & $\mathrm{~N}$ & 41 & 409 & 38.690 & $\mathrm{CB}$ & 49 \\
\hline 357 & 4.292 & HA2 & 42 & 410 & 53.520 & CA & 49 \\
\hline
\end{tabular}




\begin{tabular}{|c|c|c|c|c|c|c|c|}
\hline 411 & 118.700 & $\mathrm{~N}$ & 49 & 464 & 3.731 & $\mathrm{HA}$ & 57 \\
\hline 412 & 2.615 & HB3 & 50 & 465 & 3.150 & HD2 & 57 \\
\hline 413 & 3.285 & HB2 & 50 & 466 & 3.150 & HD3 & 57 \\
\hline 414 & 4.930 & $\mathrm{HA}$ & 50 & 467 & 8.420 & $\mathrm{H}$ & 57 \\
\hline 415 & 7.014 & $\mathrm{HE} 2$ & 50 & 468 & 28.220 & CG & 57 \\
\hline 416 & 9.930 & $\mathrm{H}$ & 50 & 469 & 30.400 & $\mathrm{CB}$ & 57 \\
\hline 417 & 25.503 & HD2 & 50 & 470 & 43.720 & $\mathrm{CD}$ & 57 \\
\hline 418 & 42.350 & $\mathrm{CB}$ & 50 & 471 & 59.680 & $\mathrm{CA}$ & 57 \\
\hline 419 & 55.920 & $\mathrm{CA}$ & 50 & 472 & 122.100 & $\mathrm{~N}$ & 57 \\
\hline 420 & 123.800 & $\mathrm{~N}$ & 50 & 473 & 2.082 & HB3 & 58 \\
\hline 421 & 3.880 & HB2 & 51 & 474 & 2.082 & HB2 & 58 \\
\hline 422 & 3.949 & HB3 & 51 & 475 & 2.447 & HG2 & 58 \\
\hline 423 & 4.607 & $\mathrm{HA}$ & 51 & 476 & 2.447 & HG 3 & 58 \\
\hline 424 & 9.390 & $\mathrm{H}$ & 51 & 477 & 4.009 & $\mathrm{HA}$ & 58 \\
\hline 425 & 64.070 & $\mathrm{CB}$ & 51 & 478 & 8.000 & $\mathrm{H}$ & 58 \\
\hline 426 & 59.960 & $\mathrm{CA}$ & 51 & 479 & 28.960 & $\mathrm{CB}$ & 58 \\
\hline 427 & 116.000 & $\mathrm{~N}$ & 51 & 480 & 34.190 & $\mathrm{CG}$ & 58 \\
\hline 428 & 1.353 & HG2 & 52 & 481 & 57.970 & $\mathrm{CA}$ & 58 \\
\hline 429 & 1.639 & HG 3 & 52 & 482 & 115.300 & $\mathrm{~N}$ & 58 \\
\hline 430 & 1.759 & HB3 & 52 & 483 & 1.054 & HG2 & 59 \\
\hline 431 & 1.919 & HB2 & 52 & 484 & 4.082 & $\mathrm{HA}$ & 59 \\
\hline 432 & 3.256 & HD2 & 52 & 485 & 3.954 & $\mathrm{HB}$ & 59 \\
\hline 433 & 3.260 & HD3 & 52 & 486 & 7.350 & $\mathrm{H}$ & 59 \\
\hline 434 & 4.609 & $\mathrm{HA}$ & 52 & 487 & 22.330 & CG2 & 59 \\
\hline 435 & 7.430 & $\mathrm{H}$ & 52 & 488 & 65.770 & $\mathrm{CA}$ & 59 \\
\hline 436 & 54.260 & CA & 52 & 489 & 69.370 & $\mathrm{CB}$ & 59 \\
\hline 437 & 116.800 & $\mathrm{~N}$ & 52 & 490 & 112.200 & $\mathrm{~N}$ & 59 \\
\hline 438 & 1.094 & HG2 & 53 & 491 & 1.849 & HG & 60 \\
\hline 439 & 4.106 & $\mathrm{HA}$ & 53 & 492 & 2.106 & HB2 & 60 \\
\hline 440 & 4.352 & HB & 53 & 493 & 2.106 & HB3 & 60 \\
\hline 441 & 8.128 & $\mathrm{H}$ & 53 & 494 & 4.684 & $\mathrm{HA}$ & 60 \\
\hline 442 & 2.780 & HB3 & 54 & 495 & 7.730 & $\mathrm{H}$ & 60 \\
\hline 443 & 2.846 & HB2 & 54 & 496 & 41.040 & $\mathrm{CB}$ & 60 \\
\hline 444 & 4.543 & $\mathrm{HA}$ & 54 & 497 & 53.390 & $\mathrm{CA}$ & 60 \\
\hline 445 & 7.967 & $\mathrm{H}$ & 54 & 498 & 112.000 & $\mathrm{~N}$ & 60 \\
\hline 446 & 2.884 & HB3 & 55 & 499 & 1.965 & HB3 & 61 \\
\hline 447 & 3.073 & HB2 & 55 & 500 & 2.119 & HB2 & 61 \\
\hline 448 & 4.425 & $\mathrm{HA}$ & 55 & 501 & 2.145 & HG2 & 61 \\
\hline 449 & 6.830 & $\mathrm{H}$ & 55 & 502 & 2.227 & HG 3 & 61 \\
\hline 450 & 40.620 & $\mathrm{CB}$ & 55 & 503 & 3.810 & $\mathrm{HA}$ & 61 \\
\hline 451 & 57.000 & $\mathrm{CA}$ & 55 & 504 & 7.340 & $\mathrm{H}$ & 61 \\
\hline 452 & 118.000 & $\mathrm{~N}$ & 55 & 505 & 30.440 & $\mathrm{CB}$ & 61 \\
\hline 453 & 2.967 & HB2 & 56 & 506 & 33.780 & $\mathrm{CG}$ & 61 \\
\hline 454 & 2.511 & HB3 & 56 & 507 & 58.280 & $\mathrm{CA}$ & 61 \\
\hline 455 & 2.012 & HG & 56 & 508 & 120.800 & $\mathrm{~N}$ & 61 \\
\hline 456 & 6.710 & $\mathrm{H}$ & 56 & 509 & 2.940 & HB2 & 62 \\
\hline 457 & 43.860 & $\mathrm{CB}$ & 56 & 510 & 3.077 & HB3 & 62 \\
\hline 458 & 58.950 & $\mathrm{CA}$ & 56 & 511 & 4.764 & $\mathrm{HA}$ & 62 \\
\hline 459 & 120.400 & $\mathrm{~N}$ & 56 & 512 & 6.769 & $\mathrm{HE} 2$ & 62 \\
\hline 460 & 1.527 & HG2 & 57 & 513 & 6.897 & HD2 & 62 \\
\hline 461 & 1.640 & HG3 & 57 & 514 & 8.190 & $\mathrm{H}$ & 62 \\
\hline 462 & 1.742 & HB3 & 57 & 515 & 55.390 & $\mathrm{CA}$ & 62 \\
\hline 463 & 1.742 & HB2 & 57 & 516 & 119.600 & $\mathrm{~N}$ & 62 \\
\hline
\end{tabular}




$\begin{array}{rlllrrll}517 & 1.904 & \text { HG2 } & 63 & 528 & 62.020 & \text { CA } & 64 \\ 518 & 1.948 & \text { HG3 } & 63 & 529 & 33.360 & \text { CB } & 64 \\ 519 & 1.963 & \text { HB3 } & 63 & 530 & 21.340 & \text { CG1 } & 64 \\ 520 & 2.242 & \text { HB2 } & 63 & 531 & 20.050 & \text { CG2 } & 64 \\ 521 & 3.633 & \text { HD2 } & 63 & 532 & 119.200 & \text { N } & 64 \\ 522 & 3.787 & \text { HD3 } & 63 & 533 & 3.963 & \text { HA2 } & 65 \\ 523 & 4.365 & \text { HA } & 63 & 534 & 4.094 & \text { HA3 } & 65 \\ 524 & 0.9327 & \text { HG2 } & 64 & 535 & 7.938 & \text { H } & 65 \\ 525 & 2.102 & \text { HB } & 64 & 536 & 46.200 & \text { CA } & 65 \\ 526 & 4.187 & \text { HA } & 64 & 537 & 117.600 & \text { N } & 65 \\ 527 & 8.058 & \text { H } & 64 & & & & \end{array}$




\section{Appendix D}

\section{NMR restraints for Conk-S2}

\section{D.1 Distance restraints for Conk-S2}

\begin{tabular}{|c|c|c|c|c|c|c|}
\hline 25 & ARG & $\mathrm{H}$ & 40 & TYR & $\mathrm{H}$ & 4.92 \\
\hline 16 & SER & HA & 40 & TYR & $\mathrm{H}$ & 5.19 \\
\hline 24 & GLN & $\mathrm{HA}$ & 40 & TYR & $\mathrm{H}$ & 5.46 \\
\hline 39 & THR & $\mathrm{HA}$ & 40 & TYR & $\mathrm{H}$ & 2.91 \\
\hline 22 & PRO & HA & 40 & TYR & $\mathrm{H}$ & 4.91 \\
\hline 39 & THR & $\mathrm{HB}$ & 40 & TYR & $\mathrm{H}$ & 4.53 \\
\hline 40 & TYR & $\mathrm{H}$ & 40 & TYR & HB3 & 3.41 \\
\hline 40 & TYR & $\mathrm{H}$ & 40 & TYR & HB2 & 3.62 \\
\hline 24 & GLN & HA & 25 & ARG & $\mathrm{H}$ & 2.70 \\
\hline 25 & ARG & $\mathrm{H}$ & 39 & THR & $\mathrm{HA}$ & 4.95 \\
\hline 24 & GLN & HG2 & 25 & ARG & $\mathrm{H}$ & 5.20 \\
\hline 24 & GLN & HG 3 & 25 & ARG & $\mathrm{H}$ & 4.65 \\
\hline 24 & GLN & HB3 & 25 & ARG & $\mathrm{H}$ & 3.95 \\
\hline 25 & ARG & $\mathrm{H}$ & 37 & THR & QG2 & 4.69 \\
\hline 29 & ASN & $\mathrm{H}$ & 34 & GLN & $\tilde{\mathrm{H}}$ & 3.95 \\
\hline 29 & ASN & $\mathrm{H}$ & 35 & CYSS & $\mathrm{HA}$ & 3.90 \\
\hline 29 & ASN & $\mathrm{H}$ & 34 & GLN & $\mathrm{HA}$ & 4.82 \\
\hline 29 & ASN & $\mathrm{H}$ & 36 & VAL & QG2 & 3.88 \\
\hline 27 & TYR & $\mathrm{H}$ & 37 & THR & $\mathrm{HA}$ & 5.00 \\
\hline 26 & ILE & HA & 27 & TYR & $\mathrm{H}$ & 2.93 \\
\hline 27 & TYR & $\mathrm{H}$ & 27 & TYR & HB3 & 3.48 \\
\hline 26 & ILE & QG2 & 27 & TYR & $\mathrm{H}$ & 3.42 \\
\hline 27 & TYR & $\mathrm{H}$ & 38 & PHE & $\mathrm{H}$ & 5.50 \\
\hline 27 & TYR & $\mathrm{H}$ & 36 & VAL & $\mathrm{H}$ & 3.71 \\
\hline 27 & TYR & $\mathrm{H}$ & 27 & TYR & HD2 & 3.15 \\
\hline 27 & TYR & $\mathrm{H}$ & 36 & VAL & QG2 & 4.45 \\
\hline 30 & SER & $\mathrm{H}$ & 31 & ALA & $\mathrm{H}$ & 3.96 \\
\hline 31 & $A L A$ & $\mathrm{H}$ & 31 & $A L A$ & $\mathrm{QB}$ & 3.11 \\
\hline 40 & TYR & $\mathrm{H}$ & 41 & ASN & $\mathrm{H}$ & 4.33 \\
\hline 38 & PHE & $\mathrm{HE} 2$ & 41 & ASN & $\mathrm{H}$ & 5.19 \\
\hline 40 & TYR & HD2 & 41 & ASN & $\mathrm{H}$ & 5.50 \\
\hline 40 & TYR & $\mathrm{HA}$ & 41 & ASN & $\mathrm{H}$ & 3.02 \\
\hline 16 & SER & HA & 41 & ASN & $\mathrm{H}$ & 3.39 \\
\hline 40 & TYR & HB2 & 41 & ASN & $\mathrm{H}$ & 4.58 \\
\hline 26 & ILE & $\mathrm{H}$ & 50 & PHE & $\mathrm{H}$ & 3.88 \\
\hline 50 & PHE & $\mathrm{H}$ & 50 & PHE & $\mathrm{HE} 2$ & 5.50 \\
\hline 49 & ASN & HA & 50 & PHE & $\mathrm{H}$ & 3.01 \\
\hline 50 & PHE & $\mathrm{H}$ & 50 & PHE & HB2 & 3.64 \\
\hline 25 & ARG & HB3 & 50 & PHE & $\mathrm{H}$ & 4.44 \\
\hline 26 & ILE & HB & 50 & PHE & $\mathrm{H}$ & 4.06 \\
\hline 28 & TYR & $\mathrm{H}$ & 50 & PHE & $\mathrm{HE} 2$ & 4.18 \\
\hline 27 & TYR & HA & 28 & TYR & $\mathrm{H}$ & 3.26 \\
\hline 28 & TYR & $\mathrm{H}$ & 28 & TYR & HB3 & 3.40 \\
\hline 27 & TYR & HB2 & 28 & TYR & $\mathrm{H}$ & 3.59 \\
\hline
\end{tabular}




\begin{tabular}{|c|c|c|c|c|c|c|}
\hline 28 & TYR & $\mathrm{H}$ & 28 & TYR & HB2 & 3.70 \\
\hline 21 & LYS & $\mathrm{H}$ & 42 & GLY & $\mathrm{H}$ & 5.50 \\
\hline 20 & THR & HB & 21 & LYS & $\mathrm{H}$ & 3.60 \\
\hline 19 & GLY & HA2 & 21 & LYS & $\mathrm{H}$ & 4.11 \\
\hline 35 & CYSS & HB2 & 36 & VAL & $\mathrm{H}$ & 5.50 \\
\hline 35 & CYSS & HB3 & 36 & VAL & $\mathrm{H}$ & 5.50 \\
\hline 36 & VAL & $\mathrm{H}$ & 36 & VAL & HB & 3.90 \\
\hline 26 & ILE & QG2 & 36 & VAL & $\mathrm{H}$ & 3.85 \\
\hline 36 & VAL & $\mathrm{H}$ & 36 & VAL & QG2 & 3.52 \\
\hline 34 & GLN & HA & 35 & CYSS & $\mathrm{H}$ & 3.16 \\
\hline 35 & CYSS & $\mathrm{H}$ & 36 & VAL & $\mathrm{H}$ & 5.03 \\
\hline 57 & ARG & $\mathrm{H}$ & 58 & GLN & $\mathrm{H}$ & 3.74 \\
\hline 56 & CYSS & $\mathrm{H}$ & 57 & ARG & $\mathrm{H}$ & 3.62 \\
\hline 54 & ASN & HA & 57 & ARG & $\mathrm{H}$ & 4.45 \\
\hline 56 & CYSS & HB3 & 57 & ARG & $\mathrm{H}$ & 4.11 \\
\hline 57 & ARG & $\mathrm{H}$ & 61 & GLN & HG3 & 4.20 \\
\hline 57 & ARG & $\mathrm{H}$ & 57 & ARG & HB2 & 3.18 \\
\hline 57 & ARG & $\mathrm{H}$ & 57 & ARG & HG2 & 3.69 \\
\hline 16 & SER & $\mathrm{H}$ & 17 & GLY & $\mathrm{H}$ & 3.96 \\
\hline 15 & ASP & HA & 16 & SER & $\mathrm{H}$ & 3.04 \\
\hline 16 & SER & $\mathrm{H}$ & 16 & SER & HB2 & 3.68 \\
\hline 15 & ASP & HB3 & 16 & SER & $\mathrm{H}$ & 5.50 \\
\hline 5 & ASP & $\mathrm{H}$ & 6 & ARG & $\mathrm{H}$ & 4.58 \\
\hline 5 & ASP & $\mathrm{HA}$ & 6 & ARG & $\mathrm{H}$ & 2.70 \\
\hline 5 & ASP & HB2 & 6 & ARG & $\mathrm{H}$ & 4.33 \\
\hline 6 & ARG & $\mathrm{H}$ & 6 & ARG & HB3 & 3.70 \\
\hline 5 & ASP & HB3 & 6 & ARG & $\mathrm{H}$ & 4.33 \\
\hline 11 & ASN & HB3 & 12 & LEU & $\mathrm{H}$ & 4.23 \\
\hline 11 & ASN & HB2 & 12 & LEU & $\mathrm{H}$ & 3.54 \\
\hline 12 & LEU & $\mathrm{H}$ & 12 & LEU & QD1 & 3.35 \\
\hline 11 & ASN & $\mathrm{H}$ & 12 & LEU & $\mathrm{H}$ & 3.43 \\
\hline 9 & TYR & HD2 & 12 & LEU & $\mathrm{H}$ & 5.48 \\
\hline 9 & TYR & $\mathrm{HA}$ & 12 & LEU & $\mathrm{H}$ & 3.88 \\
\hline 12 & LEU & $\mathrm{H}$ & 48 & ASN & HB2 & 5.50 \\
\hline 12 & LEU & $\mathrm{H}$ & 12 & LEU & HB2 & 3.64 \\
\hline 12 & LEU & $\mathrm{H}$ & 12 & LEU & HG & 3.93 \\
\hline 23 & GLU & $\mathrm{HA}$ & 24 & GLN & $\mathrm{H}$ & 2.73 \\
\hline 24 & GLN & $\mathrm{H}$ & 24 & GLN & HG2 & 5.50 \\
\hline 24 & GLN & $\mathrm{H}$ & 24 & GLN & HG3 & 3.36 \\
\hline 24 & GLN & $\mathrm{H}$ & 24 & GLN & HB2 & 2.92 \\
\hline 14 & ALA & $\mathrm{HA}$ & 15 & ASP & $\mathrm{H}$ & 2.96 \\
\hline 15 & ASP & $\mathrm{H}$ & 15 & ASP & HB2 & 3.63 \\
\hline 15 & ASP & $\mathrm{H}$ & 15 & ASP & HB3 & 3.74 \\
\hline 13 & PRO & HA & 15 & ASP & $\mathrm{H}$ & 5.50 \\
\hline 43 & LYS & $\mathrm{H}$ & 44 & GLY & $\mathrm{H}$ & 4.81 \\
\hline 19 & GLY & $\mathrm{H}$ & 43 & LYS & $\mathrm{H}$ & 4.87 \\
\hline 43 & LYS & $\mathrm{H}$ & 43 & LYS & $\mathrm{HA}$ & 2.85 \\
\hline 17 & GLY & HA 3 & 43 & LYS & $\mathrm{H}$ & 3.36 \\
\hline 43 & LYS & $\mathrm{H}$ & 43 & LYS & HG2 & 4.03 \\
\hline 29 & ASN & HB2 & 30 & SER & $\mathrm{H}$ & 4.40 \\
\hline 29 & ASN & $\mathrm{H}$ & 30 & SER & $\mathrm{H}$ & 5.50 \\
\hline 29 & ASN & $\mathrm{HA}$ & 30 & SER & $\mathrm{H}$ & 3.46 \\
\hline 29 & ASN & HB3 & 30 & SER & $\mathrm{H}$ & 3.67 \\
\hline
\end{tabular}




\begin{tabular}{|c|c|c|c|c|c|c|}
\hline 4 & LYS & $\mathrm{H}$ & 4 & LYS & HB2 & 3.76 \\
\hline 25 & ARG & $\mathrm{H}$ & 38 & PHE & $\mathrm{H}$ & 3.92 \\
\hline 27 & TYR & HD2 & 38 & PHE & $\mathrm{H}$ & 3.49 \\
\hline 24 & GLN & $\mathrm{HA}$ & 38 & PHE & $\mathrm{H}$ & 4.43 \\
\hline 37 & THR & HB & 38 & PHE & $\mathrm{H}$ & 3.72 \\
\hline 37 & THR & QG2 & 38 & PHE & $\mathrm{H}$ & 4.11 \\
\hline 23 & GLU & $\mathrm{H}$ & 40 & TYR & $\mathrm{H}$ & 3.61 \\
\hline 23 & GLU & $\mathrm{H}$ & 24 & GLN & $\mathrm{H}$ & 4.48 \\
\hline 23 & GLU & $\mathrm{H}$ & 39 & THR & $\mathrm{HA}$ & 5.13 \\
\hline 23 & GLU & $\mathrm{H}$ & 40 & TYR & HB3 & 4.82 \\
\hline 23 & GLU & $\mathrm{H}$ & 23 & GLU & HG2 & 4.71 \\
\hline 23 & GLU & $\mathrm{H}$ & 23 & GLU & HG3 & 4.71 \\
\hline 23 & GLU & $\mathrm{H}$ & 23 & GLU & HB2 & 3.90 \\
\hline 23 & GLU & $\mathrm{H}$ & 39 & THR & HG 1 & 4.63 \\
\hline 60 & CYSS & $\mathrm{H}$ & 61 & GLN & $\mathrm{H}$ & 3.99 \\
\hline 61 & GLN & $\mathrm{H}$ & 61 & GLN & HB2 & 3.13 \\
\hline 61 & GLN & $\mathrm{H}$ & 62 & TYR & HB2 & 4.90 \\
\hline 13 & PRO & $\mathrm{HA}$ & 14 & $A L A$ & $\mathrm{H}$ & 3.00 \\
\hline 40 & TYR & $\mathrm{HE} 2$ & 48 & ASN & $\mathrm{H}$ & 5.39 \\
\hline 40 & TYR & HD2 & 48 & ASN & $\mathrm{H}$ & 5.50 \\
\hline 9 & TYR & $\mathrm{HA}$ & 48 & ASN & $\mathrm{H}$ & 5.50 \\
\hline 48 & ASN & $\mathrm{H}$ & 48 & ASN & HB2 & 3.42 \\
\hline 12 & LEU & QD1 & 48 & ASN & $\mathrm{H}$ & 5.26 \\
\hline 4 & LYS & $\mathrm{H}$ & 5 & ASP & $\mathrm{H}$ & 4.25 \\
\hline 4 & LYS & $\mathrm{HA}$ & 5 & ASP & $\mathrm{H}$ & 3.42 \\
\hline 4 & LYS & HB3 & 5 & ASP & $\mathrm{H}$ & 4.10 \\
\hline 56 & CYSS & $\mathrm{H}$ & 59 & THR & $\mathrm{H}$ & 5.39 \\
\hline 55 & ASP & $\mathrm{H}$ & 56 & CYSS & $\mathrm{H}$ & 3.83 \\
\hline 54 & ASN & HA & 56 & CYSS & $\mathrm{H}$ & 4.96 \\
\hline 50 & PHE & HB3 & 56 & CYSS & $\mathrm{H}$ & 4.11 \\
\hline 56 & CYSS & $\mathrm{H}$ & 56 & CYSS & HB2 & 3.76 \\
\hline 56 & CYSS & $\mathrm{H}$ & 56 & CYSS & HB3 & 3.42 \\
\hline 52 & ARG & HB2 & 56 & CYSS & $\mathrm{H}$ & 4.37 \\
\hline 62 & TYR & $\mathrm{H}$ & 62 & TYR & HB2 & 4.01 \\
\hline 62 & TYR & $\mathrm{H}$ & 62 & TYR & HB3 & 3.64 \\
\hline 61 & GLN & HB2 & 62 & TYR & $\mathrm{H}$ & 3.77 \\
\hline 26 & ILE & $\mathrm{H}$ & 27 & TYR & HD2 & 5.15 \\
\hline 25 & ARG & HA & 26 & ILE & $\mathrm{H}$ & 3.29 \\
\hline 25 & ARG & HD3 & 26 & ILE & $\mathrm{H}$ & 4.54 \\
\hline 26 & ILE & $\mathrm{H}$ & 50 & PHE & HB2 & 5.17 \\
\hline 25 & ARG & HB3 & 26 & ILE & $\mathrm{H}$ & 3.26 \\
\hline 26 & ILE & $\mathrm{H}$ & 26 & ILE & $\mathrm{HB}$ & 3.25 \\
\hline 26 & ILE & $\mathrm{H}$ & 37 & THR & QG2 & 5.50 \\
\hline 26 & ILE & $\mathrm{H}$ & 26 & ILE & HG 13 & 3.37 \\
\hline 26 & ILE & $\mathrm{H}$ & 26 & ILE & QD1 & 3.71 \\
\hline 61 & GLN & $\mathrm{H}$ & 62 & TYR & $\mathrm{H}$ & 3.72 \\
\hline 60 & CYSS & HB3 & 62 & TYR & $\mathrm{H}$ & 5.43 \\
\hline 61 & GLN & HG2 & 62 & TYR & $\mathrm{H}$ & 5.50 \\
\hline 9 & TYR & $\mathrm{H}$ & 10 & CYSS & $\mathrm{H}$ & 4.13 \\
\hline 10 & CYSS & $\mathrm{H}$ & 50 & PHE & $\mathrm{HE} 2$ & 4.59 \\
\hline 10 & CYSS & $\mathrm{H}$ & 48 & ASN & HA & 5.34 \\
\hline 7 & PRO & HD2 & 10 & CYSS & $\mathrm{H}$ & 4.56 \\
\hline 9 & TYR & HB2 & 10 & CYSS & $\mathrm{H}$ & 4.56 \\
\hline
\end{tabular}




\begin{tabular}{|c|c|c|c|c|c|c|}
\hline 6 & ARG & HB3 & 10 & CYSS & $\mathrm{H}$ & 5.50 \\
\hline 10 & CYSS & $\mathrm{H}$ & 12 & LEU & QD1 & 4.60 \\
\hline 63 & PRO & HA & 64 & VAL & $\mathrm{H}$ & 3.11 \\
\hline 64 & VAL & $\mathrm{H}$ & 64 & VAL & $\mathrm{HB}$ & 4.01 \\
\hline 64 & VAL & $\mathrm{H}$ & 64 & VAL & QG1 & 3.76 \\
\hline 45 & GLY & HA2 & 46 & ASN & $\mathrm{H}$ & 3.24 \\
\hline 15 & ASP & HB2 & 46 & ASN & $\mathrm{H}$ & 3.88 \\
\hline 40 & TYR & HD2 & 49 & ASN & $\mathrm{H}$ & 5.50 \\
\hline 49 & ASN & $\mathrm{H}$ & 50 & PHE & $\mathrm{HE} 2$ & 5.50 \\
\hline 48 & ASN & HA & 49 & ASN & $\mathrm{H}$ & 3.12 \\
\hline 49 & ASN & $\mathrm{H}$ & 49 & ASN & HB3 & 3.56 \\
\hline 50 & PHE & HB3 & 55 & ASP & $\mathrm{H}$ & 5.18 \\
\hline 55 & ASP & $\mathrm{H}$ & 55 & ASP & HB2 & 3.34 \\
\hline 52 & ARG & HB2 & 55 & ASP & $\mathrm{H}$ & 3.80 \\
\hline 52 & ARG & HB3 & 55 & ASP & $\mathrm{H}$ & 3.96 \\
\hline 18 & SER & $\mathrm{H}$ & 43 & LYS & $\mathrm{H}$ & 4.44 \\
\hline 17 & GLY & HA3 & 18 & SER & $\mathrm{H}$ & 2.99 \\
\hline 18 & SER & $\mathrm{H}$ & 44 & GLY & HA3 & 5.10 \\
\hline 17 & GLY & HA2 & 18 & SER & $\mathrm{H}$ & 3.13 \\
\hline 18 & SER & $\mathrm{H}$ & 43 & LYS & HD2 & 5.37 \\
\hline 18 & SER & $\mathrm{H}$ & 43 & LYS & HD3 & 5.37 \\
\hline 64 & VAL & HA & 65 & GLY & $\mathrm{H}$ & 3.53 \\
\hline 64 & VAL & HB & 65 & GLY & $\mathrm{H}$ & 4.09 \\
\hline 64 & VAL & QG1 & 65 & GLY & $\mathrm{H}$ & 3.83 \\
\hline 38 & PHE & $\mathrm{H}$ & 39 & THR & $\mathrm{H}$ & 5.22 \\
\hline 38 & PHE & HD2 & 39 & THR & $\mathrm{H}$ & 4.00 \\
\hline 38 & PHE & $\mathrm{HA}$ & 39 & THR & $\mathrm{H}$ & 3.13 \\
\hline 39 & THR & $\mathrm{H}$ & 39 & THR & $\mathrm{HB}$ & 3.18 \\
\hline 38 & PHE & HB3 & 39 & THR & $\mathrm{H}$ & 3.74 \\
\hline 38 & PHE & HB2 & 39 & THR & $\mathrm{H}$ & 3.34 \\
\hline 39 & THR & $\mathrm{H}$ & 39 & THR & HG 1 & 4.27 \\
\hline 52 & ARG & $\mathrm{H}$ & 55 & ASP & $\mathrm{H}$ & 5.07 \\
\hline 51 & SER & HB3 & 52 & ARG & $\mathrm{H}$ & 4.46 \\
\hline 50 & PHE & HB3 & 52 & ARG & $\mathrm{H}$ & 3.59 \\
\hline 52 & ARG & $\mathrm{H}$ & 55 & ASP & HB3 & 5.12 \\
\hline 52 & ARG & $\mathrm{H}$ & 55 & ASP & HB2 & 4.02 \\
\hline 50 & PHE & HB2 & 52 & ARG & $\mathrm{H}$ & 4.48 \\
\hline 52 & ARG & $\mathrm{H}$ & 52 & ARG & HB2 & 3.80 \\
\hline 52 & ARG & $\mathrm{H}$ & 52 & ARG & HB3 & 3.62 \\
\hline 29 & ASN & $\mathrm{H}$ & 33 & LYS & $\mathrm{H}$ & 5.12 \\
\hline 32 & LYS & $\mathrm{H}$ & 33 & LYS & $\mathrm{H}$ & 3.15 \\
\hline 33 & LYS & $\mathrm{H}$ & 34 & GLN & $\mathrm{H}$ & 3.58 \\
\hline 33 & LYS & $\mathrm{H}$ & 33 & LYS & $\mathrm{HA}$ & 2.93 \\
\hline 29 & ASN & HB3 & 33 & LYS & $\mathrm{H}$ & 4.69 \\
\hline 32 & LYS & HB2 & 33 & LYS & $\mathrm{H}$ & 4.14 \\
\hline 9 & TYR & $\mathrm{H}$ & 9 & TYR & HB2 & 3.46 \\
\hline 7 & PRO & HG2 & 9 & TYR & $\mathrm{H}$ & 5.01 \\
\hline 9 & TYR & $\mathrm{H}$ & 12 & LEU & HG & 5.50 \\
\hline 9 & TYR & $\mathrm{H}$ & 12 & LEU & QD1 & 4.41 \\
\hline 51 & SER & $\mathrm{H}$ & 52 & ARG & $\mathrm{H}$ & 3.95 \\
\hline 51 & SER & $\mathrm{H}$ & 51 & SER & HB3 & 3.67 \\
\hline 50 & PHE & HB3 & 51 & SER & $\mathrm{H}$ & 4.33 \\
\hline 58 & GLN & $\mathrm{H}$ & 60 & CYSS & $\mathrm{H}$ & 4.67 \\
\hline
\end{tabular}




\begin{tabular}{|c|c|c|c|c|c|c|}
\hline 54 & ASN & $\mathrm{HA}$ & 58 & GLN & $\mathrm{H}$ & 4.61 \\
\hline 55 & ASP & HA & 58 & GLN & $\mathrm{H}$ & 5.13 \\
\hline 58 & GLN & $\mathrm{H}$ & 58 & GLN & HB2 & 3.15 \\
\hline 57 & ARG & HB2 & 58 & GLN & $\mathrm{H}$ & 3.16 \\
\hline 31 & ALA & $\mathrm{H}$ & 32 & LYS & $\mathrm{H}$ & 3.09 \\
\hline 32 & LYS & $\mathrm{H}$ & 34 & GLN & $\mathrm{H}$ & 5.21 \\
\hline 32 & LYS & $\mathrm{H}$ & 33 & LYS & $\mathrm{HA}$ & 5.11 \\
\hline 32 & LYS & $\mathrm{H}$ & 32 & LYS & HB2 & 3.93 \\
\hline 31 & ALA & QB & 32 & LYS & $\mathrm{H}$ & 3.43 \\
\hline 32 & LYS & $\mathrm{H}$ & 32 & LYS & HG 3 & 3.41 \\
\hline 32 & LYS & $\mathrm{H}$ & 32 & LYS & HG2 & 3.26 \\
\hline 26 & ILE & HA & 37 & THR & $\mathrm{H}$ & 5.50 \\
\hline 36 & VAL & HA & 37 & THR & $\mathrm{H}$ & 2.99 \\
\hline 36 & VAL & HB & 37 & THR & $\mathrm{H}$ & 3.17 \\
\hline 37 & THR & $\mathrm{H}$ & 37 & THR & QG2 & 3.57 \\
\hline 36 & VAL & QG2 & 37 & THR & $\mathrm{H}$ & 4.04 \\
\hline 29 & ASN & HB3 & 34 & GLN & $\mathrm{H}$ & 4.29 \\
\hline 58 & GLN & $\mathrm{H}$ & 59 & THR & $\mathrm{H}$ & 3.61 \\
\hline 55 & ASP & HA & 59 & THR & $\mathrm{H}$ & 4.86 \\
\hline 59 & THR & $\mathrm{H}$ & 59 & THR & HB & 2.94 \\
\hline 58 & GLN & HG2 & 59 & THR & $\mathrm{H}$ & 5.50 \\
\hline 58 & GLN & HG3 & 59 & THR & $\mathrm{H}$ & 5.50 \\
\hline 58 & GLN & HB2 & 59 & THR & $\mathrm{H}$ & 3.58 \\
\hline 59 & THR & $\mathrm{H}$ & 60 & CYSS & $\mathrm{H}$ & 3.08 \\
\hline 60 & CYSS & $\mathrm{H}$ & 60 & CYSS & HB2 & 3.75 \\
\hline 11 & ASN & $\mathrm{H}$ & 12 & LEU & HB2 & 4.76 \\
\hline 11 & ASN & $\mathrm{H}$ & 28 & TYR & HD2 & 5.50 \\
\hline 7 & PRO & HD2 & 11 & ASN & $\mathrm{H}$ & 5.36 \\
\hline 11 & ASN & $\mathrm{H}$ & 11 & ASN & HB2 & 3.15 \\
\hline 7 & PRO & HG2 & 11 & ASN & $\mathrm{H}$ & 4.50 \\
\hline 11 & ASN & $\mathrm{H}$ & 12 & LEU & HG & 4.85 \\
\hline 15 & ASP & $\mathrm{HA}$ & 17 & GLY & $\mathrm{H}$ & 4.38 \\
\hline 16 & SER & HB2 & 17 & GLY & $\mathrm{H}$ & 4.11 \\
\hline 43 & LYS & $\mathrm{HA}$ & 44 & GLY & $\mathrm{H}$ & 2.73 \\
\hline 43 & LYS & HG2 & 44 & GLY & $\mathrm{H}$ & 4.96 \\
\hline 43 & LYS & HG 3 & 44 & GLY & $\mathrm{H}$ & 5.26 \\
\hline 20 & THR & $\mathrm{H}$ & 21 & LYS & $\mathrm{H}$ & 3.85 \\
\hline 20 & THR & $\mathrm{H}$ & 20 & THR & QG2 & 4.12 \\
\hline 19 & GLY & $\mathrm{H}$ & 19 & GLY & HA2 & 2.78 \\
\hline 24 & GLN & $\mathrm{HA}$ & 37 & THR & $\mathrm{HB}$ & 3.80 \\
\hline 24 & GLN & HB3 & 37 & THR & $\mathrm{HB}$ & 4.29 \\
\hline 24 & GLN & HG2 & 37 & THR & HB & 4.60 \\
\hline 24 & GLN & HG3 & 37 & THR & $\mathrm{HB}$ & 5.15 \\
\hline 16 & SER & $\mathrm{HA}$ & 39 & THR & HB & 4.99 \\
\hline 59 & THR & HB & 61 & GLN & HG2 & 5.50 \\
\hline 58 & GLN & HB2 & 59 & THR & HA & 4.96 \\
\hline 58 & GLN & HB3 & 59 & THR & $\mathrm{HA}$ & 5.49 \\
\hline 59 & THR & $\mathrm{HA}$ & 60 & CYSS & HB2 & 5.50 \\
\hline 16 & SER & HB3 & 40 & TYR & HA & 4.60 \\
\hline 16 & SER & HB2 & 40 & TYR & $\mathrm{HA}$ & 4.82 \\
\hline 16 & SER & $\mathrm{HA}$ & 16 & SER & HB2 & 2.93 \\
\hline 16 & SER & HB2 & 17 & GLY & HA3 & 5.09 \\
\hline 25 & ARG & HD2 & 51 & SER & HB3 & 5.50 \\
\hline
\end{tabular}




\begin{tabular}{|c|c|c|c|c|c|c|}
\hline 24 & GLN & HA & 39 & THR & HA & 4.16 \\
\hline 39 & THR & HA & 40 & TYR & HB3 & 4.72 \\
\hline 39 & THR & HA & 39 & THR & HG1 & 3.18 \\
\hline 64 & VAL & HA & 64 & VAL & QG1 & 2.40 \\
\hline 54 & ASN & HA & 57 & ARG & HA & 5.05 \\
\hline 57 & ARG & HA & 61 & GLN & HG3 & 3.01 \\
\hline 57 & ARG & $\mathrm{HA}$ & 57 & ARG & HG3 & 3.07 \\
\hline 28 & TYR & HA & 61 & GLN & $\mathrm{HA}$ & 5.18 \\
\hline 60 & CYSS & $\mathrm{HA}$ & 61 & GLN & $\mathrm{HA}$ & 4.76 \\
\hline 61 & GLN & $\mathrm{HA}$ & 62 & TYR & $\mathrm{HA}$ & 5.18 \\
\hline 28 & TYR & HA & 28 & TYR & HB2 & 2.55 \\
\hline 4 & LYS & HA & 4 & LYS & HB3 & 2.55 \\
\hline 31 & ALA & QB & 32 & LYS & HA & 4.22 \\
\hline 32 & LYS & HA & 32 & LYS & HG3 & 3.62 \\
\hline 32 & LYS & HA & 32 & LYS & HG2 & 3.49 \\
\hline 14 & ALA & HA & 15 & ASP & $\mathrm{HA}$ & 4.78 \\
\hline 13 & PRO & HA & 14 & ALA & HA & 4.57 \\
\hline 14 & ALA & HA & 48 & ASN & HB2 & 4.95 \\
\hline 14 & ALA & HA & 38 & PHE & HB2 & 5.50 \\
\hline 14 & ALA & HA & 15 & ASP & HB2 & 5.26 \\
\hline 43 & LYS & $\mathrm{HA}$ & 44 & GLY & HA2 & 4.54 \\
\hline 17 & GLY & HA3 & 44 & GLY & HA 3 & 5.50 \\
\hline 15 & ASP & HB2 & 48 & ASN & $\mathrm{HA}$ & 5.50 \\
\hline 15 & ASP & HB3 & 16 & SER & $\mathrm{HA}$ & 5.33 \\
\hline 50 & PHE & HB3 & 56 & CYSS & HB3 & 4.20 \\
\hline 56 & CYSS & HB2 & 61 & GLN & HG3 & 4.29 \\
\hline 56 & CYSS & HB2 & 61 & GLN & HG2 & 4.45 \\
\hline 26 & ILE & HB & 56 & CYSS & HB3 & 5.21 \\
\hline 15 & ASP & HB2 & 16 & SER & HA & 5.22 \\
\hline 15 & ASP & HB2 & 45 & GLY & HA2 & 5.24 \\
\hline 15 & ASP & HB2 & 45 & GLY & HA 3 & 3.91 \\
\hline 15 & ASP & HB3 & 45 & GLY & HA 3 & 4.26 \\
\hline 19 & GLY & HA2 & 43 & LYS & HG2 & 5.39 \\
\hline 16 & SER & HB2 & 19 & GLY & HA2 & 4.98 \\
\hline 19 & GLY & HA2 & 43 & LYS & HG3 & 5.50 \\
\hline 25 & ARG & $\mathrm{HA}$ & 25 & ARG & HD3 & 3.03 \\
\hline 25 & ARG & HA & 25 & ARG & HD2 & 3.08 \\
\hline 25 & ARG & HB3 & 25 & ARG & HD2 & 3.88 \\
\hline 25 & ARG & HD2 & 26 & ILE & QD1 & 5.14 \\
\hline 50 & PHE & HB2 & 52 & ARG & HB2 & 5.50 \\
\hline 40 & TYR & HB3 & 41 & ASN & $\mathrm{HA}$ & 5.50 \\
\hline 16 & SER & HA & 40 & TYR & HB2 & 4.98 \\
\hline 25 & ARG & HG3 & 40 & TYR & HB3 & 3.65 \\
\hline 27 & TYR & HB3 & 49 & ASN & HA & 4.35 \\
\hline 14 & ALA & $\mathrm{HA}$ & 27 & TYR & HB2 & 5.50 \\
\hline 14 & ALA & $\mathrm{HA}$ & 27 & TYR & HB3 & 5.50 \\
\hline 27 & TYR & HB2 & 28 & TYR & HB3 & 5.50 \\
\hline 27 & TYR & HB3 & 38 & PHE & HB3 & 5.14 \\
\hline 52 & ARG & HB2 & 55 & ASP & HB3 & 4.39 \\
\hline 12 & LEU & QD1 & 46 & ASN & HB3 & 3.66 \\
\hline 50 & PHE & HB3 & 55 & ASP & HB3 & 4.16 \\
\hline 26 & ILE & $\mathrm{HB}$ & 50 & PHE & HB2 & 3.62 \\
\hline 52 & ARG & HB2 & 55 & ASP & HB2 & 5.50 \\
\hline
\end{tabular}




\begin{tabular}{|c|c|c|c|c|c|c|}
\hline 12 & LEU & QD1 & 46 & ASN & HB2 & 3.66 \\
\hline 26 & ILE & $\mathrm{HB}$ & 26 & ILE & QD1 & 3.96 \\
\hline 7 & PRO & HG2 & 28 & TYR & HB3 & 5.48 \\
\hline 29 & ASN & HB3 & 32 & LYS & HG2 & 4.87 \\
\hline 10 & CYSS & HB3 & 28 & TYR & HB2 & 3.00 \\
\hline 7 & PRO & HG2 & 10 & CYSS & HB2 & 5.23 \\
\hline 6 & ARG & HB3 & 10 & CYSS & HB2 & 4.39 \\
\hline 9 & TYR & HB3 & 48 & ASN & $\mathrm{HA}$ & 3.89 \\
\hline 9 & TYR & HB3 & 12 & LEU & HB2 & 5.50 \\
\hline 7 & PRO & HG2 & 9 & TYR & HB2 & 4.41 \\
\hline 9 & TYR & HB3 & 12 & LEU & QD1 & 4.00 \\
\hline 9 & TYR & HB2 & 12 & LEU & QD1 & 4.52 \\
\hline 13 & PRO & HA & 48 & ASN & HB2 & 5.50 \\
\hline 12 & LEU & HB2 & 48 & ASN & HB2 & 4.68 \\
\hline 34 & GLN & HB2 & 36 & VAL & QG1 & 4.61 \\
\hline 34 & GLN & HB3 & 36 & VAL & QG1 & 4.61 \\
\hline 23 & GLU & HB2 & 40 & TYR & HB3 & 3.58 \\
\hline 61 & GLN & $\mathrm{HA}$ & 61 & GLN & HG2 & 3.27 \\
\hline 32 & LYS & HB3 & 32 & LYS & HG2 & 2.74 \\
\hline 60 & CYSS & $\mathrm{HA}$ & 61 & GLN & HG2 & 4.54 \\
\hline 60 & CYSS & $\mathrm{HA}$ & 61 & GLN & HG3 & 5.50 \\
\hline 32 & LYS & HB2 & 32 & LYS & HG3 & 2.40 \\
\hline 57 & ARG & $\mathrm{HA}$ & 61 & GLN & HB2 & 3.80 \\
\hline 54 & ASN & $\mathrm{HA}$ & 57 & ARG & HB2 & 2.91 \\
\hline 35 & CYSS & HB2 & 61 & GLN & HB2 & 5.50 \\
\hline 35 & CYSS & HB3 & 61 & GLN & HB2 & 5.50 \\
\hline 24 & GLN & HB3 & 24 & GLN & HG2 & 2.41 \\
\hline 25 & ARG & HG2 & 40 & TYR & HB3 & 3.65 \\
\hline 32 & LYS & $\mathrm{HA}$ & 32 & LYS & HD3 & 4.05 \\
\hline 58 & GLN & HB2 & 59 & THR & QG2 & 5.50 \\
\hline 57 & ARG & HB3 & 57 & ARG & HG2 & 2.40 \\
\hline 25 & ARG & $\mathrm{HA}$ & 26 & ILE & HG 12 & 5.50 \\
\hline 25 & ARG & HA & 26 & ILE & HG 13 & 4.42 \\
\hline 57 & ARG & $\mathrm{HA}$ & 57 & ARG & HG2 & 3.76 \\
\hline 26 & ILE & QG2 & 26 & ILE & HG 13 & 3.65 \\
\hline 26 & ILE & HG13 & 37 & THR & QG2 & 3.80 \\
\hline 26 & ILE & QG2 & 26 & ILE & HG 12 & 3.68 \\
\hline 29 & ASN & HB3 & 32 & LYS & HG3 & 3.59 \\
\hline 43 & LYS & $\mathrm{HA}$ & 43 & LYS & HG2 & 3.77 \\
\hline 29 & ASN & HB2 & 32 & LYS & HG3 & 4.77 \\
\hline 18 & SER & $\mathrm{HA}$ & 43 & LYS & HG 3 & 4.93 \\
\hline 28 & TYR & HB2 & 59 & THR & QG2 & 5.00 \\
\hline 36 & VAL & HA & 37 & THR & QG2 & 4.21 \\
\hline 36 & VAL & $\mathrm{HA}$ & 36 & VAL & QG1 & 3.97 \\
\hline 24 & GLN & HG2 & 37 & THR & QG2 & 4.78 \\
\hline 36 & VAL & HB & 37 & THR & QG2 & 4.55 \\
\hline 24 & GLN & HB3 & 37 & THR & QG2 & 4.31 \\
\hline 26 & ILE & HG 12 & 37 & THR & QG2 & 4.49 \\
\hline 36 & VAL & QG2 & 37 & THR & QG2 & 4.58 \\
\hline 20 & THR & QG2 & 43 & LYS & HA & 5.38 \\
\hline 19 & GLY & HA2 & 20 & THR & QG2 & 5.50 \\
\hline 26 & ILE & QG2 & 56 & CYSS & HB2 & 3.72 \\
\hline 26 & ILE & QG2 & 50 & $\mathrm{PHE}$ & HB2 & 3.62 \\
\hline
\end{tabular}




\begin{tabular}{|c|c|c|c|c|c|c|}
\hline 26 & ILE & QG2 & 56 & CYSS & HB3 & 3.54 \\
\hline 26 & ILE & QG2 & 26 & ILE & QD1 & 2.85 \\
\hline 36 & VAL & HA & 36 & VAL & QG2 & 4.03 \\
\hline 63 & PRO & $\mathrm{HA}$ & 64 & VAL & QG1 & 4.92 \\
\hline 62 & TYR & HB2 & 64 & VAL & QG1 & 5.26 \\
\hline 62 & TYR & HB3 & 64 & VAL & QG 1 & 5.50 \\
\hline 29 & ASN & HB3 & 36 & VAL & QG2 & 3.90 \\
\hline 32 & LYS & HB2 & 36 & VAL & QG2 & 5.49 \\
\hline 32 & LYS & HD2 & 36 & VAL & QG2 & 5.50 \\
\hline 56 & CYSS & HB2 & 57 & ARG & HG2 & 5.50 \\
\hline 27 & TYR & HB2 & 48 & ASN & HB2 & 5.50 \\
\hline 27 & TYR & HB3 & 48 & ASN & HB2 & 5.50 \\
\hline 26 & ILE & QD1 & 37 & THR & $\mathrm{HB}$ & 5.50 \\
\hline 38 & PHE & HB2 & 39 & THR & HB & 5.50 \\
\hline 20 & THR & HA & 20 & THR & QG2 & 3.16 \\
\hline 10 & CYSS & $\mathrm{HA}$ & 10 & CYSS & HB3 & 2.54 \\
\hline 9 & TYR & $\mathrm{HA}$ & 9 & TYR & HB3 & 2.55 \\
\hline 10 & CYSS & $\mathrm{HA}$ & 28 & TYR & HB3 & 3.52 \\
\hline 9 & TYR & $\mathrm{HA}$ & 12 & LEU & QD1 & 3.09 \\
\hline 55 & ASP & HA & 55 & ASP & HB3 & 2.55 \\
\hline 55 & ASP & HA & 58 & GLN & HB2 & 3.48 \\
\hline 38 & PHE & HA & 38 & PHE & HB3 & 2.94 \\
\hline 11 & ASN & HA & 30 & SER & HA & 4.81 \\
\hline 59 & THR & $\mathrm{HA}$ & 60 & CYSS & HA & 5.12 \\
\hline 37 & THR & $\mathrm{H}$ & 37 & THR & HB & 3.99 \\
\hline 59 & THR & HB & 60 & CYSS & $\mathrm{H}$ & 3.32 \\
\hline 18 & SER & HB2 & 19 & GLY & $\mathrm{H}$ & 5.11 \\
\hline 18 & SER & HB3 & 19 & GLY & $\mathrm{H}$ & 5.11 \\
\hline 16 & SER & HB3 & 17 & GLY & $\mathrm{H}$ & 5.13 \\
\hline 16 & SER & $\mathrm{H}$ & 16 & SER & HB3 & 3.78 \\
\hline 16 & SER & HB2 & 41 & ASN & $\mathrm{H}$ & 4.30 \\
\hline 16 & SER & HB3 & 38 & PHE & $\mathrm{HE} 2$ & 4.63 \\
\hline 16 & SER & HB2 & 38 & PHE & $\mathrm{HE} 2$ & 5.50 \\
\hline 28 & TYR & HD2 & 30 & SER & $\mathrm{HA}$ & 4.88 \\
\hline 57 & ARG & HA & 62 & TYR & $\mathrm{H}$ & 5.28 \\
\hline 57 & ARG & HA & 60 & CYSS & $\mathrm{H}$ & 4.92 \\
\hline 57 & ARG & $\mathrm{HA}$ & 61 & GLN & $\mathrm{H}$ & 3.83 \\
\hline 33 & LYS & $\mathrm{HA}$ & 34 & GLN & $\mathrm{H}$ & 3.39 \\
\hline 45 & GLY & HA3 & 46 & ASN & $\mathrm{H}$ & 3.52 \\
\hline 57 & ARG & $\mathrm{H}$ & 57 & ARG & HD2 & 5.50 \\
\hline 57 & ARG & $\mathrm{H}$ & 57 & ARG & HD3 & 5.50 \\
\hline 56 & CYSS & HB2 & 57 & ARG & $\mathrm{H}$ & 3.66 \\
\hline 15 & ASP & HB3 & 46 & ASN & $\mathrm{H}$ & 4.98 \\
\hline 17 & GLY & $\mathrm{H}$ & 45 & GLY & HA3 & 4.95 \\
\hline 50 & PHE & HB2 & 56 & CYSS & $\mathrm{H}$ & 4.83 \\
\hline 38 & PHE & $\mathrm{HE} 2$ & 40 & TYR & HB3 & 4.28 \\
\hline 40 & TYR & HB3 & 41 & ASN & $\mathrm{H}$ & 5.50 \\
\hline 40 & TYR & HB3 & 42 & GLY & $\mathrm{H}$ & 5.27 \\
\hline 38 & PHE & $\mathrm{HE} 2$ & 40 & TYR & HB2 & 4.89 \\
\hline 27 & TYR & HB3 & 38 & PHE & $\mathrm{H}$ & 5.37 \\
\hline 27 & TYR & HB3 & 27 & TYR & HD2 & 3.0 \\
\hline 27 & TYR & HB2 & 50 & PHE & $\mathrm{HE} 2$ & 4.4 \\
\hline 27 & TYR & HB3 & 27 & TYR & HE2 & 5.13 \\
\hline
\end{tabular}




\begin{tabular}{|c|c|c|c|c|c|c|}
\hline 27 & TYR & HE2 & 38 & PHE & HB2 & 4.75 \\
\hline 38 & PHE & HB2 & 38 & PHE & $\mathrm{HE} 2$ & 5.26 \\
\hline 38 & PHE & $\mathrm{H}$ & 38 & PHE & HB2 & 4.06 \\
\hline 38 & PHE & $\mathrm{H}$ & 38 & PHE & HB3 & 3.84 \\
\hline 60 & CYSS & HB3 & 61 & GLN & $\mathrm{H}$ & 3.67 \\
\hline 38 & PHE & HB2 & 38 & PHE & HD2 & 3.31 \\
\hline 27 & TYR & $\mathrm{HE} 2$ & 38 & PHE & HB3 & 3.68 \\
\hline 46 & ASN & HB2 & 47 & GLY & $\mathrm{H}$ & 3.36 \\
\hline 46 & ASN & HB3 & 47 & GLY & $\mathrm{H}$ & 3.36 \\
\hline 26 & ILE & $\mathrm{HB}$ & 27 & TYR & HD2 & 5.50 \\
\hline 55 & ASP & $\mathrm{H}$ & 55 & ASP & HB3 & 3.89 \\
\hline 55 & ASP & HB3 & 56 & CYSS & $\mathrm{H}$ & 4.25 \\
\hline 55 & ASP & HB2 & 56 & CYSS & $\mathrm{H}$ & 3.85 \\
\hline 11 & ASN & $\mathrm{H}$ & 11 & ASN & HB3 & 3.72 \\
\hline 29 & ASN & $\mathrm{H}$ & 29 & ASN & HB3 & 3.68 \\
\hline 28 & TYR & HB3 & 28 & TYR & HD2 & 3.41 \\
\hline 28 & TYR & HD2 & 29 & ASN & HB2 & 5.50 \\
\hline 27 & TYR & $\mathrm{HA}$ & 28 & TYR & HB2 & 5.50 \\
\hline 28 & TYR & HB2 & 35 & CYSS & HA & 5.50 \\
\hline 28 & TYR & HB2 & 28 & TYR & HD2 & 3.65 \\
\hline 40 & TYR & HD2 & 49 & ASN & HB3 & 4.91 \\
\hline 49 & ASN & $\mathrm{H}$ & 49 & ASN & HB2 & 3.56 \\
\hline 27 & TYR & HA & 28 & TYR & HB3 & 4.85 \\
\hline 10 & CYSS & $\mathrm{H}$ & 10 & CYSS & HB2 & 3.75 \\
\hline 10 & CYSS & HB3 & 28 & TYR & HD2 & 2.97 \\
\hline 40 & TYR & HD2 & 49 & ASN & HB2 & 4.91 \\
\hline 9 & TYR & HB3 & 50 & PHE & HE2 & 3.58 \\
\hline 9 & TYR & HB2 & 50 & PHE & $\mathrm{HE} 2$ & 3.48 \\
\hline 27 & TYR & $\mathrm{HE} 2$ & 36 & VAL & HB & 5.02 \\
\hline 25 & ARG & HB2 & 27 & TYR & HD2 & 4.87 \\
\hline 25 & ARG & HB2 & 40 & TYR & HD2 & 5.40 \\
\hline 25 & ARG & HB3 & 40 & TYR & HD2 & 5.50 \\
\hline 32 & LYS & HB2 & 34 & GLN & $\mathrm{H}$ & 4.02 \\
\hline 32 & LYS & HB3 & 34 & GLN & $\mathrm{H}$ & 4.61 \\
\hline 56 & CYSS & $\mathrm{H}$ & 57 & ARG & HB2 & 5.40 \\
\hline 25 & ARG & HG2 & 40 & TYR & $\mathrm{H}$ & 4.77 \\
\hline 25 & ARG & HG 3 & 40 & TYR & $\mathrm{H}$ & 4.77 \\
\hline 32 & LYS & $\mathrm{H}$ & 32 & LYS & HD2 & 5.05 \\
\hline 32 & LYS & $\mathrm{H}$ & 32 & LYS & HD3 & 5.47 \\
\hline 57 & ARG & HG2 & 58 & GLN & $\mathrm{H}$ & 5.50 \\
\hline 26 & ILE & $\mathrm{H}$ & 26 & ILE & HG12 & 4.75 \\
\hline 26 & ILE & HG 12 & 37 & THR & HA & 5.14 \\
\hline 26 & ILE & HG 13 & 37 & THR & HA & 4.37 \\
\hline 31 & ALA & $\mathrm{H}$ & 32 & LYS & HG 3 & 4.27 \\
\hline 19 & GLY & $\mathrm{H}$ & 43 & LYS & HG 3 & 4.28 \\
\hline 36 & VAL & $\mathrm{H}$ & 36 & VAL & QG1 & 5.10 \\
\hline 27 & TYR & $\mathrm{HE} 2$ & 36 & VAL & QG1 & 5.10 \\
\hline 35 & CYSS & $\mathrm{HA}$ & 36 & VAL & QG1 & 4.59 \\
\hline 20 & THR & QG2 & 21 & LYS & $\mathrm{H}$ & 5.43 \\
\hline 26 & ILE & $\mathrm{H}$ & 26 & ILE & QG2 & 4.96 \\
\hline 26 & ILE & QG2 & 27 & TYR & HD2 & 5.01 \\
\hline 26 & ILE & OG2 & 56 & CYSS & $\mathrm{H}$ & 5.50 \\
\hline 26 & ILE & QG2 & 37 & THR & HA & 5.22 \\
\hline
\end{tabular}




\begin{tabular}{|c|c|c|c|c|c|c|}
\hline 26 & ILE & $\mathrm{HA}$ & 26 & ILE & QG2 & 3.28 \\
\hline 27 & TYR & HD2 & 36 & VAL & QG2 & 4.70 \\
\hline 27 & TYR & $\mathrm{HE} 2$ & 36 & VAL & QG2 & 3.64 \\
\hline 28 & TYR & HA & 29 & ASN & $\mathrm{H}$ & 2.95 \\
\hline 28 & TYR & HA & 36 & VAL & $\mathrm{H}$ & 4.19 \\
\hline 10 & CYSS & $\mathrm{HA}$ & 50 & PHE & HE2 & 3.82 \\
\hline 46 & ASN & $\mathrm{HA}$ & 47 & GLY & $\mathrm{H}$ & 3.08 \\
\hline 27 & TYR & HD2 & 38 & PHE & HB3 & 3.92 \\
\hline 37 & THR & HA & 37 & THR & QG2 & 3.37 \\
\hline 36 & VAL & QG2 & 37 & THR & HA & 4.00 \\
\hline 37 & THR & HA & 38 & PHE & $\mathrm{HA}$ & 4.74 \\
\hline 36 & VAL & HA & 37 & THR & $\mathrm{HA}$ & 4.64 \\
\hline 26 & ILE & HA & 37 & THR & $\mathrm{HA}$ & 3.05 \\
\hline 27 & TYR & HD2 & 37 & THR & $\mathrm{HA}$ & 4.40 \\
\hline 25 & ARG & $\mathrm{H}$ & 37 & THR & $\mathrm{HA}$ & 5.32 \\
\hline 37 & THR & HA & 38 & PHE & $\mathrm{H}$ & 2.92 \\
\hline 26 & ILE & HA & 26 & ILE & QD1 & 3.90 \\
\hline 26 & ILE & HA & 37 & THR & QG2 & 4.51 \\
\hline 26 & ILE & HA & 26 & ILE & HG13 & 4.05 \\
\hline 26 & ILE & HA & 27 & TYR & HB3 & 5.26 \\
\hline 25 & ARG & HA & 26 & ILE & HA & 4.75 \\
\hline 26 & ILE & HA & 37 & THR & HB & 4.51 \\
\hline 26 & ILE & HA & 27 & TYR & HD2 & 4.06 \\
\hline 26 & ILE & $\mathrm{HA}$ & 38 & PHE & $\mathrm{H}$ & 4.40 \\
\hline 35 & CYSS & $\mathrm{HA}$ & 36 & VAL & $\mathrm{H}$ & 2.80 \\
\hline 28 & TYR & $\mathrm{HA}$ & 35 & CYSS & HA & 3.01 \\
\hline 26 & ILE & QG2 & 35 & CYSS & $\mathrm{HA}$ & 4.41 \\
\hline 35 & CYSS & HA & 36 & VAL & QG2 & 4.30 \\
\hline 18 & SER & HA & 19 & GLY & $\mathrm{H}$ & 3.26 \\
\hline 38 & PHE & $\mathrm{HA}$ & 38 & PHE & HD2 & 4.64 \\
\hline 50 & PHE & HA & 51 & SER & $\mathrm{H}$ & 3.11 \\
\hline 27 & TYR & HA & 27 & TYR & HB2 & 2.96 \\
\hline 26 & ILE & QG2 & 27 & TYR & HA & 4.34 \\
\hline 27 & TYR & HA & 50 & PHE & HE2 & 4.82 \\
\hline 27 & TYR & HA & 27 & TYR & HD2 & 4.12 \\
\hline 4 & LYS & $\mathrm{H}$ & 4 & LYS & QG & 4.83 \\
\hline 4 & LYS & $\mathrm{HA}$ & 4 & LYS & QG & 3.54 \\
\hline 5 & ASP & $\mathrm{H}$ & 5 & ASP & QB & 3.55 \\
\hline 5 & ASP & QB & 6 & ARG & $\mathrm{H}$ & 3.75 \\
\hline 5 & ASP & QB & 6 & ARG & $\mathrm{HA}$ & 5.34 \\
\hline 8 & SER & QB & 9 & TYR & $\mathrm{H}$ & 4.04 \\
\hline 8 & SER & QB & 11 & ASN & $\mathrm{H}$ & 5.34 \\
\hline 9 & TYR & HB3 & 47 & GLY & QA & 5.34 \\
\hline 12 & LEU & QD1 & 46 & ASN & QB & 3.20 \\
\hline 18 & SER & $\mathrm{H}$ & 18 & SER & QB & 3.66 \\
\hline 18 & SER & $\mathrm{H}$ & 43 & LYS & QD & 4.50 \\
\hline 18 & SER & QB & 19 & GLY & $\mathrm{H}$ & 4.36 \\
\hline 19 & GLY & $\mathrm{H}$ & 41 & ASN & QB & 3.11 \\
\hline 23 & GLU & $\mathrm{H}$ & 23 & GLU & QG & 4.08 \\
\hline 23 & GLU & HB2 & 23 & GLU & QG & 2.58 \\
\hline 23 & GLU & HB2 & 25 & ARG & QG & 4.79 \\
\hline 23 & GLU & HB3 & 25 & ARG & QG & 5.34 \\
\hline 23 & GLU & QG & 24 & GLN & $\mathrm{H}$ & 5.34 \\
\hline
\end{tabular}




\begin{tabular}{|c|c|c|c|c|c|c|}
\hline 25 & ARG & $\mathrm{H}$ & 25 & ARG & QG & 4.03 \\
\hline 25 & ARG & HA & 25 & ARG & QG & 3.32 \\
\hline 25 & ARG & QG & 26 & ILE & $\mathrm{H}$ & 5.18 \\
\hline 25 & ARG & QG & 40 & TYR & $\mathrm{H}$ & 3.97 \\
\hline 25 & ARG & QG & 40 & TYR & HB2 & 3.48 \\
\hline 25 & ARG & QG & 40 & TYR & HB3 & 3.16 \\
\hline 26 & ILE & QG2 & 35 & CYSS & $\mathrm{QB}$ & 4.16 \\
\hline 32 & LYS & HG2 & 34 & GLN & QG & 5.28 \\
\hline 33 & LYS & $\mathrm{H}$ & 33 & LYS & QG & 3.13 \\
\hline 33 & LYS & $\mathrm{H}$ & 33 & LYS & $\mathrm{QE}$ & 5.34 \\
\hline 33 & LYS & HA & 33 & LYS & QG & 3.07 \\
\hline 33 & LYS & QB & 33 & LYS & QD & 2.59 \\
\hline 33 & LYS & QB & 33 & LYS & $\mathrm{QE}$ & 5.08 \\
\hline 33 & LYS & QG & 33 & LYS & $\mathrm{QE}$ & 2.62 \\
\hline 33 & LYS & $\mathrm{QD}$ & 33 & LYS & $\mathrm{QE}$ & 2.25 \\
\hline 34 & GLN & $\mathrm{H}$ & 34 & GLN & QB & 3.57 \\
\hline 34 & GLN & $\mathrm{H}$ & 34 & GLN & QG & 3.69 \\
\hline 34 & GLN & QB & 36 & VAL & QG1 & 3.80 \\
\hline 34 & GLN & QB & 36 & VAL & QG2 & 4.66 \\
\hline 34 & GLN & QG & 35 & CYSS & $\mathrm{H}$ & 4.40 \\
\hline 34 & GLN & QG & 36 & VAL & QG1 & 5.23 \\
\hline 35 & CYSS & $\mathrm{H}$ & 35 & CYSS & $\mathrm{QB}$ & 3.37 \\
\hline 35 & CYSS & $\mathrm{QB}$ & 36 & VAL & $\mathrm{HA}$ & 5.34 \\
\hline 40 & TYR & HE2 & 49 & ASN & QB & 4.19 \\
\hline 40 & TYR & HD2 & 49 & ASN & QB & 4.21 \\
\hline 41 & ASN & $\mathrm{H}$ & 41 & ASN & QB & 3.52 \\
\hline 43 & LYS & $\mathrm{H}$ & 43 & LYS & QB & 3.68 \\
\hline 43 & LYS & $\mathrm{HA}$ & 43 & LYS & $\mathrm{QE}$ & 4.20 \\
\hline 43 & LYS & QB & 43 & LYS & HG2 & 2.63 \\
\hline 43 & LYS & QB & 44 & GLY & $\mathrm{H}$ & 3.30 \\
\hline 43 & LYS & QD & 44 & GLY & $\mathrm{H}$ & 3.35 \\
\hline 46 & ASN & $\mathrm{H}$ & 46 & ASN & QB & 3.37 \\
\hline 46 & ASN & HA & 46 & ASN & QB & 2.47 \\
\hline 46 & ASN & QB & 47 & GLY & $\mathrm{H}$ & 2.88 \\
\hline 48 & ASN & $\mathrm{H}$ & 49 & ASN & QB & 5.34 \\
\hline 49 & ASN & $\mathrm{H}$ & 49 & ASN & QB & 3.02 \\
\hline 54 & ASN & HA & 57 & ARG & QD & 3.76 \\
\hline 54 & ASN & HA & 58 & GLN & QG & 4.27 \\
\hline 55 & ASP & HA & 58 & GLN & QG & 4.41 \\
\hline 57 & ARG & $\mathrm{HA}$ & 57 & ARG & QD & 4.55 \\
\hline 57 & ARG & HB2 & 57 & ARG & QD & 2.53 \\
\hline 57 & ARG & HB2 & 58 & GLN & QG & 5.34 \\
\hline 57 & ARG & $\mathrm{QD}$ & 58 & GLN & $\mathrm{H}$ & 5.34 \\
\hline 58 & GLN & $\mathrm{H}$ & 58 & GLN & QG & 3.65 \\
\hline 58 & GLN & $\mathrm{HA}$ & 58 & GLN & QG & 3.56 \\
\hline 58 & GLN & QG & 59 & THR & $\mathrm{H}$ & 4.74 \\
\hline 64 & VAL & $\mathrm{H}$ & 65 & GLY & QA & 5.34 \\
\hline 64 & VAL & $\mathrm{HB}$ & 65 & GLY & QA & 5.21 \\
\hline 64 & VAL & QG1 & 65 & GLY & QA & 5.26 \\
\hline
\end{tabular}


D.2 Dihedral angle restraints for Conk-S2

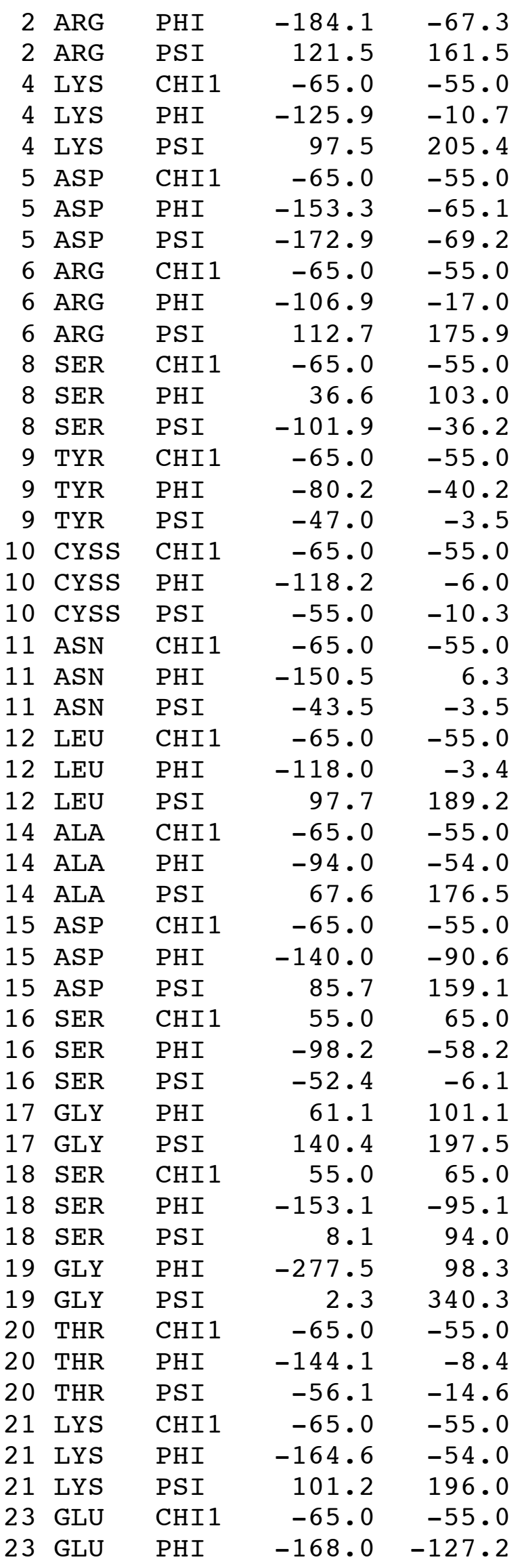




\begin{tabular}{|c|c|c|c|c|}
\hline 23 & GLU & PSI & 115.6 & 170.8 \\
\hline 24 & GLN & CHI 1 & -65.0 & -55.0 \\
\hline 24 & GLN & PHI & $-138 \cdot 7$ & -45.7 \\
\hline 24 & GLN & PSI & 94.5 & 144.6 \\
\hline 5 & ARG & CHI 1 & -65.0 & -55.0 \\
\hline 5 & ARG & PHI & -166.2 & -110.0 \\
\hline 5 & ARG & PSI & 133.7 & 211.7 \\
\hline 26 & ILE & CHI 1 & -65.0 & -55.0 \\
\hline 6 & ILE & PHI & $-158 \cdot 3$ & -105.6 \\
\hline 2 & ILE & PSI & 123.5 & 163.5 \\
\hline 27 & TYR & CHI 1 & 55.0 & 65.0 \\
\hline 27 & TYR & PHI & -157.6 & -114.1 \\
\hline 27 & TYR & PSI & 124.5 & 205.1 \\
\hline 8 & TYR & CHI 1 & 175.0 & 185.0 \\
\hline 8 & TYR & PHI & -143.9 & -41.2 \\
\hline 28 & TYR & PSI & 99.6 & 139.6 \\
\hline 29 & ASN & CHI 1 & -65.0 & -55.0 \\
\hline 29 & ASN & PHI & -126.2 & -44.0 \\
\hline 29 & ASN & PSI & 73.1 & 165.8 \\
\hline 30 & SER & CHI1 & -65.0 & -55.0 \\
\hline 0 & SER & PHI & -82.4 & -42.4 \\
\hline 30 & SER & PSI & $-81 \cdot 3$ & -29.5 \\
\hline 31 & ALA & CHI 1 & -65.0 & -55.0 \\
\hline 3 & ALA & PHI & -84.6 & $-44 \cdot 6$ \\
\hline 31 & ALA & PSI & -67.0 & -25.1 \\
\hline 32 & LYS & CHI 1 & -65.0 & -55.0 \\
\hline 32 & LYS & PHI & -87.5 & -47.5 \\
\hline 2 & LYS & PSI & -47.7 & -7.1 \\
\hline 33 & LYS & CHI 1 & -65.0 & -55.0 \\
\hline 33 & LYS & PHI & 40.6 & 80.6 \\
\hline 33 & LYS & PSI & 12.3 & 52.3 \\
\hline 34 & GLN & CHI 1 & -65.0 & -55.0 \\
\hline 34 & GLN & PHI & -175.2 & -92.9 \\
\hline 34 & GLN & PSI & 110.5 & 187.0 \\
\hline 35 & CYSS & CHI 1 & 175.0 & 185.0 \\
\hline 35 & CYSS & PHI & -133.0 & -26.6 \\
\hline 35 & CYSS & PSI & 104.6 & 165.3 \\
\hline 36 & VAL & CHI 1 & -65.0 & -55.0 \\
\hline 36 & VAL & PHI & -156.8 & -116.8 \\
\hline 3 & VAL & PSI & 116.4 & 194.1 \\
\hline 37 & THR & CHI 1 & 55.0 & 65.0 \\
\hline 37 & THR & PHI & -146.8 & -25.4 \\
\hline 37 & THR & PSI & 108.6 & 183.0 \\
\hline 38 & PHE & CHI 1 & 55.0 & 65.0 \\
\hline 3 & PHE & PHI & -199.6 & -85.0 \\
\hline 38 & PHE & PSI & 132.9 & 200.4 \\
\hline 39 & THR & $\mathrm{CHI} 1$ & -65.0 & -55.0 \\
\hline 39 & THR & PHI & -138.4 & -57.9 \\
\hline 39 & THR & PSI & 90.1 & 142.9 \\
\hline 4 & TYR & CHI 1 & 175.0 & 185.0 \\
\hline 40 & TYR & PHI & -138.8 & -73.6 \\
\hline & TYR & PSI & 87.4 & 154.4 \\
\hline & ASN & CHI 1 & -65.0 & -55.0 \\
\hline
\end{tabular}




\begin{tabular}{|c|c|c|c|c|}
\hline 41 & ASN & PHI & -93.8 & -53.8 \\
\hline 41 & ASN & PSI & -52.9 & -12.9 \\
\hline 42 & GLY & PHI & 86.7 & 126.7 \\
\hline 42 & GLY & PSI & -44.8 & 13.2 \\
\hline 43 & LYS & CHI 1 & -65.0 & -55.0 \\
\hline 43 & LYS & PHI & -133.8 & -37.9 \\
\hline 43 & LYS & PSI & $-215 \cdot 3$ & -128.1 \\
\hline 44 & GLY & PHI & -197.0 & 97.8 \\
\hline 44 & GLY & PSI & -245.4 & 83.6 \\
\hline 45 & GLY & PHI & 19.5 & 154.0 \\
\hline 45 & GLY & PSI & -218.2 & -116.8 \\
\hline 46 & ASN & CHI 1 & -65.0 & -55.0 \\
\hline 46 & ASN & PHI & -178.0 & -73.1 \\
\hline 46 & ASN & PSI & 141.2 & 193.2 \\
\hline 47 & GLY & PHI & -163.6 & 30.7 \\
\hline 47 & GLY & PSI & -267.2 & 198.0 \\
\hline 48 & ASN & CHI 1 & -185.0 & -175.0 \\
\hline 48 & ASN & PHI & -92.0 & -52.0 \\
\hline 48 & ASN & PSI & 31.7 & 146.4 \\
\hline 49 & ASN & CHI 1 & -185.0 & -175.0 \\
\hline 49 & ASN & PHI & -198.7 & -83.6 \\
\hline 49 & ASN & PSI & 84.2 & 146.8 \\
\hline 50 & PHE & CHI 1 & -65.0 & -55.0 \\
\hline 50 & PHE & PHI & -154.1 & -75.7 \\
\hline 50 & PHE & PSI & 101.4 & 166.4 \\
\hline 51 & SER & CHI 1 & 55.0 & 65.0 \\
\hline 1 & SER & PHI & -115.1 & -51.5 \\
\hline 5 & SER & PSI & -49.1 & 1.3 \\
\hline 52 & ARG & CHI 1 & -65.0 & -55.0 \\
\hline 52 & ARG & PHI & -174.4 & -85.8 \\
\hline 52 & ARG & PSI & 126.3 & 166.6 \\
\hline & THR & CHI 1 & -65.0 & -55.0 \\
\hline 5 & THR & PHI & $-122 \cdot 3$ & $-10 \cdot 6$ \\
\hline 53 & THR & PSI & -61.1 & $-18 \cdot 3$ \\
\hline 54 & ASN & CHI 1 & -65.0 & -55.0 \\
\hline 54 & ASN & PHI & -86.7 & -46.7 \\
\hline 54 & ASN & PSI & -55.4 & -15.4 \\
\hline 55 & ASP & CHI 1 & -65.0 & -55.0 \\
\hline & ASP & PHI & -89.0 & -34.1 \\
\hline & ASP & PSI & -67.9 & -23.5 \\
\hline 56 & CYSS & CHI 1 & 55.0 & 65.0 \\
\hline 56 & CYSS & PHI & -104.2 & -27.8 \\
\hline 56 & CYSS & PSI & -62.8 & -21.5 \\
\hline 57 & ARG & CHI 1 & -65.0 & -55.0 \\
\hline 5 & ARG & PHI & -84.5 & -44.5 \\
\hline 5 & ARG & PSI & -63.6 & -23.6 \\
\hline 58 & GLN & CHI 1 & -65.0 & -55.0 \\
\hline 58 & GLN & PHI & -84.7 & -44.7 \\
\hline 58 & GLN & PSI & -64.4 & -24.4 \\
\hline 59 & THR & CHI 1 & -65.0 & -55.0 \\
\hline 5 & THR & PHI & -100.1 & -29.9 \\
\hline & THR & PSI & -82.9 & -28.2 \\
\hline & CYSS & CHI 1 & -65.0 & -55.0 \\
\hline
\end{tabular}




$\begin{array}{lllrr}60 & \text { CYSS } & \text { PHI } & -256.7 & 106.3 \\ 60 & \text { CYSS } & \text { PSI } & -219.0 & 143.5 \\ 61 & \text { GLN } & \text { CHI1 } & -65.0 & -55.0 \\ 61 & \text { GLN } & \text { PHI } & -103.4 & -35.7 \\ 61 & \text { GLN } & \text { PSI } & -63.2 & -16.2 \\ 62 & \text { TYR } & \text { CHI1 } & -65.0 & -55.0 \\ 62 & \text { TYR } & \text { PHI } & -116.8 & -76.8 \\ 62 & \text { TYR } & \text { PSI } & 87.4 & 127.4 \\ 64 & \text { VAL } & \text { CHI1 } & -65.0 & -55.0 \\ 64 & \text { VAL } & \text { PHI } & -149.5 & -109.5 \\ 64 & \text { VAL } & \text { PSI } & 138.5 & 178.5\end{array}$

\section{D.3 Residual dipolar couplings for Conk-S2}

$\begin{array}{rrrrr}2 & \mathrm{~N} & 2 & \mathrm{HN} & 2.590 \\ 4 & \mathrm{~N} & 4 & \mathrm{HN} & -0.910 \\ 5 & \mathrm{~N} & 5 & \mathrm{HN} & -2.200 \\ 6 & \mathrm{~N} & 6 & \mathrm{HN} & -3.130 \\ 9 & \mathrm{~N} & 9 & \mathrm{HN} & 3.530 \\ 10 & \mathrm{~N} & 10 & \mathrm{HN} & 18.360 \\ 11 & \mathrm{~N} & 11 & \mathrm{HN} & -9.970 \\ 12 & \mathrm{~N} & 12 & \mathrm{HN} & -0.350 \\ 14 & \mathrm{~N} & 14 & \mathrm{HN} & -8.430 \\ 15 & \mathrm{~N} & 15 & \mathrm{HN} & 7.190 \\ 16 & \mathrm{~N} & 16 & \mathrm{HN} & 1.810 \\ 17 & \mathrm{~N} & 17 & \mathrm{HN} & 2.830 \\ 18 & \mathrm{~N} & 18 & \mathrm{HN} & 11.950 \\ 19 & \mathrm{~N} & 19 & \mathrm{HN} & 6.700 \\ 20 & \mathrm{~N} & 20 & \mathrm{HN} & -3.740 \\ 21 & \mathrm{~N} & 21 & \mathrm{HN} & -0.010 \\ 23 & \mathrm{~N} & 23 & \mathrm{HN} & -9.940 \\ 24 & \mathrm{~N} & 24 & \mathrm{HN} & -8.410 \\ 25 & \mathrm{~N} & 25 & \mathrm{HN} & 3.160 \\ 26 & \mathrm{~N} & 26 & \mathrm{HN} & -1.030 \\ 27 & \mathrm{~N} & 27 & \mathrm{HN} & -12.130 \\ 28 & \mathrm{~N} & 28 & \mathrm{HN} & -19.610 \\ 29 & \mathrm{~N} & 29 & \mathrm{HN} & -21.030 \\ 30 & \mathrm{~N} & 30 & \mathrm{HN} & -7.220 \\ 31 & \mathrm{~N} & 31 & \mathrm{HN} & 12.520 \\ 32 & \mathrm{~N} & 32 & \mathrm{HN} & 0.610 \\ 33 & \mathrm{~N} & 33 & \mathrm{HN} & -13.240 \\ 34 & \mathrm{~N} & 34 & \mathrm{HN} & -6.020 \\ 35 & \mathrm{~N} & 35 & \mathrm{HN} & -7.890 \\ 36 & \mathrm{~N} & 36 & \mathrm{HN} & -22.060 \\ 37 & \mathrm{~N} & 37 & \mathrm{HN} & -15.850 \\ 38 & \mathrm{~N} & 38 & \mathrm{HN} & -0.020 \\ 39 & \mathrm{~N} & 39 & \mathrm{HN} & -1.270 \\ 41 & \mathrm{~N} & 41 & \mathrm{HN} & -6.940 \\ 43 & \mathrm{~N} & 43 & \mathrm{HN} & 7.060\end{array}$




\begin{tabular}{|c|c|c|c|c|}
\hline 44 & $\mathrm{~N}$ & 44 & $\mathrm{HN}$ & 3.620 \\
\hline 45 & $\mathrm{~N}$ & 45 & $\mathrm{HN}$ & -9.850 \\
\hline 46 & $\mathrm{~N}$ & 46 & $\mathrm{HN}$ & -17.510 \\
\hline 47 & $\mathrm{~N}$ & 47 & $\mathrm{HN}$ & -11.760 \\
\hline 48 & $\mathrm{~N}$ & 48 & $\mathrm{HN}$ & -0.560 \\
\hline 49 & $\mathrm{~N}$ & 49 & $\mathrm{HN}$ & 11.340 \\
\hline 50 & $\mathrm{~N}$ & 50 & HN & 0.950 \\
\hline 51 & $\mathrm{~N}$ & 51 & $\mathrm{HN}$ & 8.370 \\
\hline 52 & $\mathrm{~N}$ & 52 & $\mathrm{HN}$ & -5.400 \\
\hline 55 & $\mathrm{~N}$ & 55 & $\mathrm{HN}$ & 17.390 \\
\hline 56 & $\mathrm{~N}$ & 56 & $\mathrm{HN}$ & -1.500 \\
\hline 57 & $\mathrm{~N}$ & 57 & $\mathrm{HN}$ & -0.500 \\
\hline 58 & $\mathrm{~N}$ & 58 & $\mathrm{HN}$ & 8.200 \\
\hline 59 & $\mathrm{~N}$ & 59 & $\mathrm{HN}$ & 13.230 \\
\hline 60 & $\mathrm{~N}$ & 60 & $\mathrm{HN}$ & -2.170 \\
\hline 61 & $\mathrm{~N}$ & 61 & $\mathrm{HN}$ & -0.330 \\
\hline 62 & $\mathrm{~N}$ & 62 & $\mathrm{HN}$ & 3.170 \\
\hline 64 & $\mathrm{~N}$ & 64 & $\mathrm{HN}$ & 2.423 \\
\hline 65 & $\mathrm{~N}$ & 65 & $\mathrm{HN}$ & -0.146 \\
\hline 2 & $\mathrm{~N}$ & 1 & C & -0.010 \\
\hline 4 & $\mathrm{~N}$ & 3 & $\mathrm{C}$ & 0.350 \\
\hline 5 & $\mathrm{~N}$ & 4 & $\mathrm{C}$ & -0.610 \\
\hline 6 & $\mathrm{~N}$ & 5 & C & 0.710 \\
\hline 9 & $\mathrm{~N}$ & 8 & C & 0.160 \\
\hline 10 & $\mathrm{~N}$ & 9 & $\mathrm{C}$ & 0.670 \\
\hline 11 & $\mathrm{~N}$ & 10 & C & -1.930 \\
\hline 12 & $\mathrm{~N}$ & 11 & $\mathrm{C}$ & 0.530 \\
\hline 14 & $\mathrm{~N}$ & 13 & $\mathrm{C}$ & -1.120 \\
\hline 15 & $\mathrm{~N}$ & 14 & $\mathrm{C}$ & -0.130 \\
\hline 16 & $\mathrm{~N}$ & 15 & C & 2.050 \\
\hline 17 & $\mathrm{~N}$ & 16 & C & -2.370 \\
\hline 18 & $\mathrm{~N}$ & 17 & C & -0.300 \\
\hline 19 & $\mathrm{~N}$ & 18 & $\mathrm{C}$ & 1.930 \\
\hline 20 & $\mathrm{~N}$ & 19 & C & -0.660 \\
\hline 21 & $\mathrm{~N}$ & 20 & C & 2.240 \\
\hline 23 & $\mathrm{~N}$ & 22 & $\mathrm{C}$ & 2.310 \\
\hline 24 & $\mathrm{~N}$ & 23 & C & -1.640 \\
\hline 25 & $\mathrm{~N}$ & 24 & C & -0.080 \\
\hline 26 & $\mathrm{~N}$ & 25 & C & 2.200 \\
\hline 27 & $\mathrm{~N}$ & 26 & $\mathrm{C}$ & -0.140 \\
\hline 28 & $\mathrm{~N}$ & 27 & C & 0.840 \\
\hline 29 & $\mathrm{~N}$ & 28 & C & -1.130 \\
\hline 30 & $\mathrm{~N}$ & 29 & C & -1.820 \\
\hline 31 & $\mathrm{~N}$ & 30 & $\mathrm{C}$ & 1.980 \\
\hline 32 & $\mathrm{~N}$ & 31 & C & -0.410 \\
\hline 33 & $\mathrm{~N}$ & 32 & C & -0.840 \\
\hline 34 & $\mathrm{~N}$ & 33 & $\mathrm{C}$ & 2.140 \\
\hline 35 & $\mathrm{~N}$ & 34 & C & -0.550 \\
\hline 36 & $\mathrm{~N}$ & 35 & $\mathrm{C}$ & 1.260 \\
\hline 37 & $\mathrm{~N}$ & 36 & C & 1.350 \\
\hline 38 & $\mathrm{~N}$ & 37 & $\mathrm{C}$ & -0.800 \\
\hline 39 & $\mathrm{~N}$ & 38 & $\mathrm{C}$ & 1.620 \\
\hline 40 & $\mathrm{~N}$ & 39 & $\mathrm{C}$ & -0.020 \\
\hline
\end{tabular}




$\begin{array}{lrrrr}41 & \mathrm{~N} & 40 & \mathrm{C} & -1.560 \\ 43 & \mathrm{~N} & 42 & \mathrm{C} & -1.330 \\ 44 & \mathrm{~N} & 43 & \mathrm{C} & 0.200 \\ 45 & \mathrm{~N} & 44 & \mathrm{C} & -0.390 \\ 46 & \mathrm{~N} & 45 & \mathrm{C} & -0.060 \\ 47 & \mathrm{~N} & 46 & \mathrm{C} & 2.390 \\ 48 & \mathrm{~N} & 47 & \mathrm{C} & 0.340 \\ 49 & \mathrm{~N} & 48 & \mathrm{C} & -0.220 \\ 50 & \mathrm{~N} & 49 & \mathrm{C} & -2.570 \\ 51 & \mathrm{~N} & 50 & \mathrm{C} & -0.310 \\ 52 & \mathrm{~N} & 51 & \mathrm{C} & 2.270 \\ 55 & \mathrm{~N} & 54 & \mathrm{C} & -0.260 \\ 56 & \mathrm{~N} & 55 & \mathrm{C} & 1.320 \\ 57 & \mathrm{~N} & 56 & \mathrm{C} & -2.530 \\ 58 & \mathrm{~N} & 57 & \mathrm{C} & -0.040 \\ 59 & \mathrm{~N} & 58 & \mathrm{C} & 1.110 \\ 60 & \mathrm{~N} & 59 & \mathrm{C} & 0.350 \\ 61 & \mathrm{~N} & 60 & \mathrm{C} & -2.330 \\ 62 & \mathrm{~N} & 61 & \mathrm{C} & 0.440 \\ 65 & \mathrm{~N} & 64 & \mathrm{C} & 0.248 \\ 39 & \mathrm{CA} & 39 & \mathrm{C} & 5.288 \\ 24 & \mathrm{CA} & 24 & \mathrm{C} & -3.474 \\ 28 & \mathrm{CA} & 28 & \mathrm{C} & -0.965 \\ 26 & \mathrm{CA} & 26 & \mathrm{C} & 0.871 \\ 40 & \mathrm{CA} & 40 & \mathrm{C} & -1.961 \\ 27 & \mathrm{CA} & 27 & \mathrm{C} & -0.23 \\ 20 & \mathrm{CA} & 20 & \mathrm{C} & 4.383 \\ 34 & \mathrm{CA} & 34 & \mathrm{C} & 1.157 \\ 56 & \mathrm{CA} & 56 & \mathrm{C} & -3.51 \\ 15 & \mathrm{CA} & 15 & \mathrm{C} & -2.628 \\ 5 & \mathrm{CA} & 5 & \mathrm{C} & -1.326 \\ 23 & \mathrm{CA} & 23 & \mathrm{C} & -0.643 \\ 11 & \mathrm{CA} & 11 & \mathrm{C} & -4.836 \\ 14 & \mathrm{CA} & 14 & \mathrm{C} & 0.974 \\ 29 & \mathrm{CA} & 29 & \mathrm{C} & -2.34 \\ 37 & \mathrm{CA} & 37 & \mathrm{C} & 0.359 \\ 4 & \mathrm{CA} & 4 & \mathrm{C} & 0.882 \\ 55 & \mathrm{CA} & 55 & \mathrm{C} & 1.342 \\ 9 & \mathrm{CA} & 9 & \mathrm{C} & 5.043 \\ 45 & \mathrm{CA} & 45 & \mathrm{C} & -3.58 \\ 17 & \mathrm{CA} & 17 & \mathrm{C} & -4.063 \\ 51 & \mathrm{CA} & 51 & \mathrm{C} & 4.442 \\ 32 & \mathrm{CA} & 32 & \mathrm{C} & -2.945 \\ 50 & \mathrm{CA} & 50 & \mathrm{C} & 3.137 \\ 31 & \mathrm{CA} & 31 & \mathrm{C} & 4.115 \\ 58 & \mathrm{CA} & 36 & \mathrm{C} & 0.142 \\ & \mathrm{CA} & 58 & \mathrm{C} & 2.675 \\ & \mathrm{CA} & 59 & \mathrm{C} & 1.795 \\ 5 & \mathrm{CA} & 10 & \mathrm{C} & -2.097 \\ 5 & & & \mathrm{C} & 0.075\end{array}$




\section{Curriculum Vitae}

\section{Personal data}

Name

Date of birth

Place of birth

Citizenship

\section{Education}

05/1996

05/1998

06/1998-05/2001

08/2001-05/2003

05/2003-08/2003

08/2003-11/2003

11/2003-05/2004

06/2004-02/2008
Jegannath Korukottu

30.03.1980

Madurai, India

Indian
Secondary School Education, Madurai, India

Higher Secondary School Education, Madurai, India

Bachelor's of Science (Bsc.) Physics (spl.), Madurai Kamaraj

University, Madurai, India.

Master's of Science (MSc.) Physics, Indian Institute of Technology Madras (IIT M), Chennai, India, Thesis titled "Quantum version of Bertrand's theorem".

Visiting student at the Institute of Mathematical Sciences, Chennai, India.

Visiting student at the Center for Bioinformatics, University of Saarbrücken, Germany Funded by EMBO

Praktikum in the group of Prof. Dr. Stefan Hell and Dr. Markus Zweckstetter at the Max Planck Institute for Biophysical Chemistry in Göttingen, Germany

Ph.D. thesis with the title "Rapid Determination of Highresolution Protein Structures by Solution and Solid-state NMR spectroscopy" in the department of NMR-based structural biology under the supervision of Prof. Dr. Christian Griesinger and Dr. Markus Zweckstetter at the Max Planck Institute for Biophysical Chemistry in Göttingen, Germany 


\section{Lebenslauf}

\section{Persönliche Daten}

Name

Geburtstag

Geburtsort

Staatsangehörigkeit
Jegannath Korukottu

30.03.1980

Madurai, Indien

Indisch

\section{Ausbildung}

05/1996

05/1998

06/1998-05/2001

08/2001-05/2003

05/2003-08/2003

$08 / 2003-11 / 2003$

11/2003-05/2004

06/2004-02/2008
Abschluss der Sekundarstufe I (10. Klasse) in Madurai, Indien Erwerb des hochschulqualifierenden Abschlusses in Madurai, Indien

Bachelor's of Science (Bsc.) in Physik an der Madurai Kamaraj Universität in Madurai, Indien.

Master's of Science (MSc.) in Physik am Indischen Institut für Technologie Madras (IIT M) in Chennai, Indien, Titel der Master-Arbeit: "Quantenmechanische Äquivalenz von Bertrands Theorem".

Gaststudent am Institut für Mathematik in Chennai, Indien

Gaststudent am Zentrum für Bioinformatik an der Universität Saarbrücken, unterstützt durch ein EMBO Stipendium Praktikum in der Gruppe von Prof. Dr. Stefan Hell und der Gruppe von Dr. Markus Zweckstetter am Max-Planck-Institut für biophysikalische Chemie in Göttingen, Germany Doktorarbeit mit dem Titel "Schnelle Bestimmung von hochaufgelösten Strukturen durch Lösungs- und FestkörperNMR-Spektroskopie“ unter Anleitung von Prof. Dr. Christian Griesinger und Dr. Markus Zweckstetter am Max Planck Institut für biophysikalische Chemie in Göttingen, Germany 